University of Louisville

ThinkIR: The University of Louisville's Institutional Repository

Electronic Theses and Dissertations

$5-2020$

\title{
Volatile organic compound exposure and cardiometabolic syndrome risk in a nationally representative cohort.
}

Stacey Lane Konkle

University of Louisville

Follow this and additional works at: https://ir.library.louisville.edu/etd

Part of the Cardiovascular Diseases Commons, Environmental Health Commons, Epidemiology

Commons, Nutritional and Metabolic Diseases Commons, and the Other Pharmacology, Toxicology and

Environmental Health Commons

\section{Recommended Citation}

Konkle, Stacey Lane, "Volatile organic compound exposure and cardiometabolic syndrome risk in a nationally representative cohort." (2020). Electronic Theses and Dissertations. Paper 3409.

https://doi.org/10.18297/etd/3409

This Doctoral Dissertation is brought to you for free and open access by ThinkIR: The University of Louisville's Institutional Repository. It has been accepted for inclusion in Electronic Theses and Dissertations by an authorized administrator of ThinkIR: The University of Louisville's Institutional Repository. This title appears here courtesy of the author, who has retained all other copyrights. For more information, please contact thinkir@louisville.edu. 


\title{
VOLATILE ORGANIC COMPOUND EXPOSURE AND CARDIOMETABOLIC SYNDROME RISK IN A NATIONALLY REPRESENTATIVE COHORT
}

\author{
By \\ Stacey Lane Konkle \\ B.S., Purdue University, 2011 \\ M.P.H., University of Louisville, 2014

\begin{abstract}
A Dissertation
Submitted to the Faculty of the

School of Public Health and Information Sciences of the University of Louisville in Partial Fulfillment of the Requirements

for the Degree of
\end{abstract} \\ Doctor of Philosophy \\ in Public Health Sciences \\ Department of Epidemiology and Population Health \\ University of Louisville \\ Louisville, Kentucky
}

May 2020 

VOLATILE ORGANIC COMPOUND EXPOSURE AND CARDIOMETABOLIC SYNDROME RISK IN A NATIONALLY REPRESENTATIVE COHORT

\author{
By \\ Stacey Lane Konkle \\ B.S., Purdue University, 2011 \\ M.P.H., University of Louisville, 2014
}

A Dissertation Approved on

March 20, 2020

By the following Dissertation Committee

Dr. Kristina Zierold

Dr. Kira Taylor

Dr. Matthew Groenewold

Dr. Aruni Bhatnagar

Dr. Shesh Rai

Dr. Stephanie Boone 


\section{ACKNOWLEDGMENTS}

First and foremost, I want to thank my family for their continued love and support. I would not be where I am today without you. Thank you for your patience and encouragement throughout my doctoral coursework and dissertation. I am very fortunate to have such a wonderful family, who never fail to cheer me on.

A special, heartfelt thank you is extended to Dr. Aruni Bhatnagar, my boss and mentor. I have thoroughly enjoyed working under your leadership. This opportunity has allowed me to expand on the knowledge that I learned from traditional coursework. You have been an exemplary researcher, advisor, and mentor, for which I am grateful. An additional heartfelt thank you is extended to Dr. Kristina Zierold and Dr. Kira Taylor, my dissertation committee co-chairs and mentors. You were my guiding light and cheerleaders. I could not have done this without your tremendous support and encouragement. Thank you, Dr. Matthew Groenewold for inspiring my love and enthusiasm for public health! I would also like to thank my committee members Dr. Stephanie Boone and Dr. Shesh Rai. I sincerely appreciate your time and valuable input. A special thanks is extended to the University of Louisville School of Public Health and information Sciences for their continued support and academic guidance throughout my time in the doctoral program. Additionally, I would like to recognize the Envirome Institute, the diabetes and obesity center, and the University of Louisville Super Fund Center for all the financial support and remarkable exposure to incredible minds and opportunities 
during my time at the University of Louisville. I would like to thank this incredible group for providing me with a Graduate Research Assistantship opportunity, I am extremely grateful for your invaluable academic and financial support.

I would like to recognize this work was supported in part by National Institute Health (NIH) grant ES 023716. 


\begin{abstract}
VOLATILE ORGANIC COMPOUND EXPOSURE AND CARDIOMETABOLIC SYNDROME RISK IN A NATIONALLY REPRESENTATIVE COHORT

Stacey L. Konkle
\end{abstract}

March 20, 2020

BACKGROUND: The relative importance of environmental exposures such as volatile organic compounds (VOCs) is one of the paramount public health priorities of our time, yet is presently unstudied. VOCs are ubiquitous in the environment and have been associated with numerous adverse health effects, including a number of cardiovascular and metabolic effects that are components of Cardiometabolic Syndrome (CMS).

OBJECTIVES: To examine the relationship between CMS and individual-level exposures to VOCs, measured as urinary metabolites of VOCs (UM-VOCs), in a nationally representative sample.

METHODS: Associations between urinary biomarkers of exposure to 19 parent VOCs and CMS were assessed using the National Health and Nutrition Examination Survey (NHANES). To isolate effects from environmental VOC exposures, analyses were stratified by tobacco-smoke exposure status. CMS was treated dichotomously as well as ordinally and associations with VOCs were considered from a single pollutant, as well as multi-pollutant perspectives. Potential important groupings and interactions among VOCs, and their 
associations with CMS were evaluated using numerous traditional regression modeling and exploratory modeling methods including: backwards-selection model-building, factor analysis, LASSO penalized regression, and a cumulative VOC exposure score.

RESULTS: Concentrations of eight UM-VOCs were significantly different between individuals with and without CMS. Among the non-smoke exposed participants, 6 UM-VOCs were significantly associated with increased odds of CMS. These associations were observed with metabolites from acrolein, 1,3-butadiene, crotonaldehyde, cyanide, and ethylbenzene/styrene. Furthermore, dose-response type relationships were observed with metabolites of acrolein, 1,3-butadiene, and crotonaldehyde. Metabolites from acrolein and ethylbenzene/styrene were associated with disease progression in ordinal logistic regression models and a cumulative VOC exposure score was significantly associated with the progression of disease towards clinically diagnosable CMS (OR: 1.015, 95\% Cl: 1.007, 1.024). DISCUSSION: This novel quantitative and nationally representative study investigated associations between individual-level exposures to VOCs and CMS. The results of this study point toward a potential causal role for certain VOCs in the development of CMS, a condition which ultimately greatly increases one's risk of the deadliest non-communicable disease in the world, cardiovascular disease. These findings are important for the development of public health interventions and policies surrounding modifiable environmental pollution exposures. 


\section{TABLE OF CONTENTS}

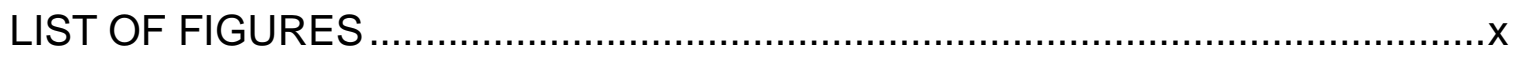

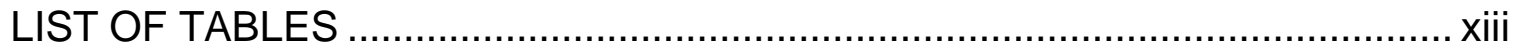

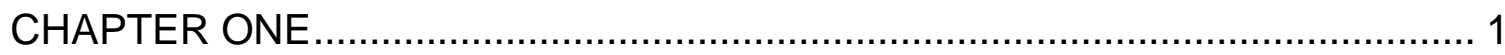

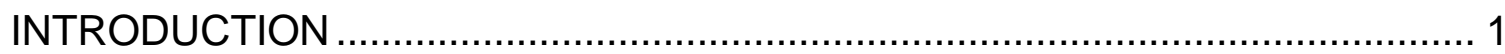

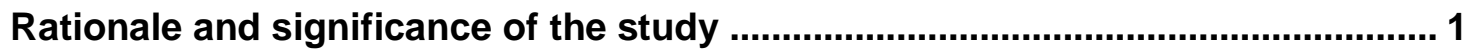

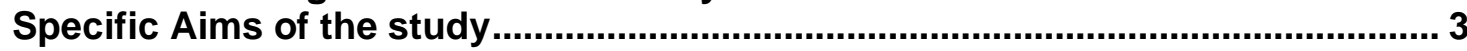

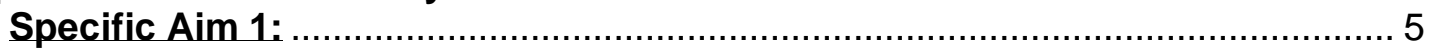

Specific Aim 2:

Specific Aim 3:

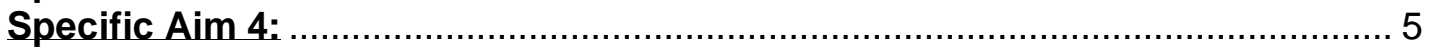

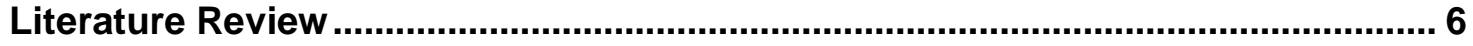

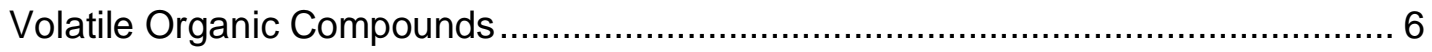

Health Effects of Volatile Organic Compounds .................................................. 9

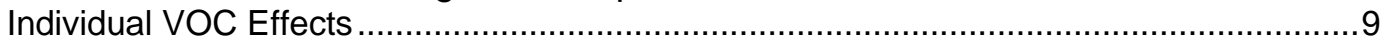

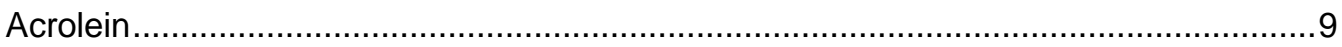

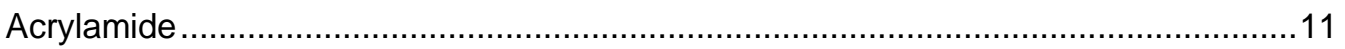

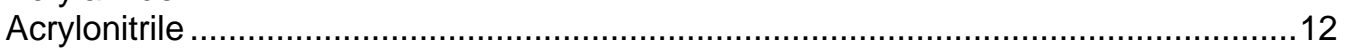

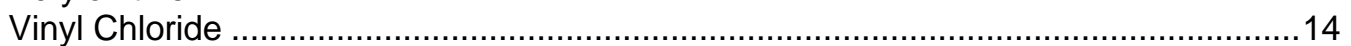

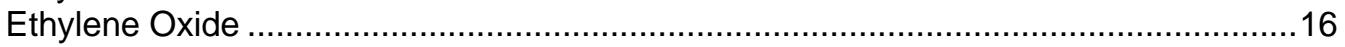

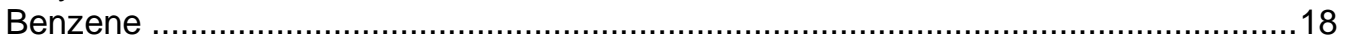

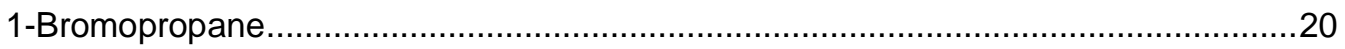

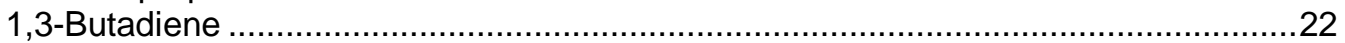

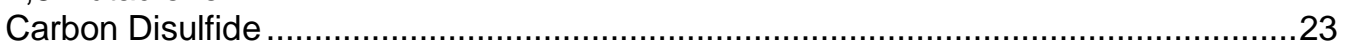

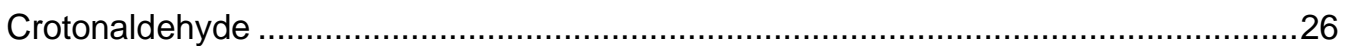

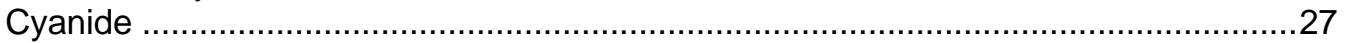

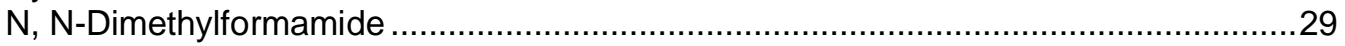

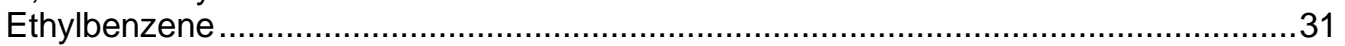

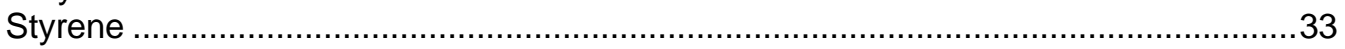

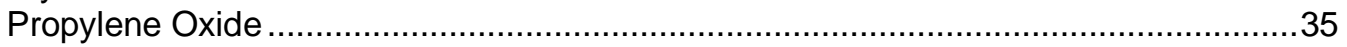

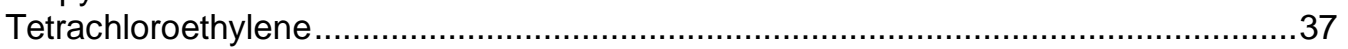

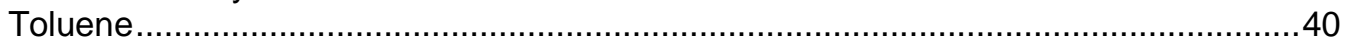

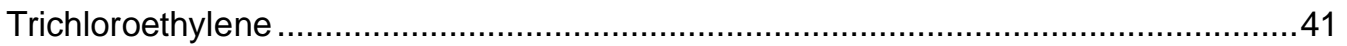

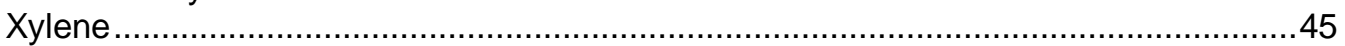

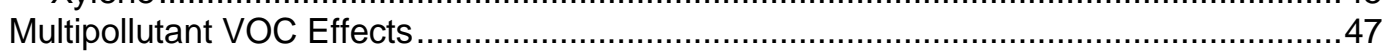

Volatile Organic Compounds and Cardiometabolic Syndrome ............................ 50

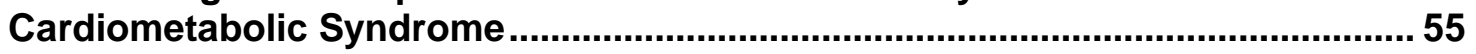

Definition of Cardiometabolic Syndrome ......................................................... 55

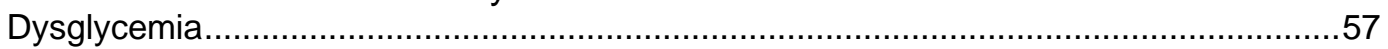

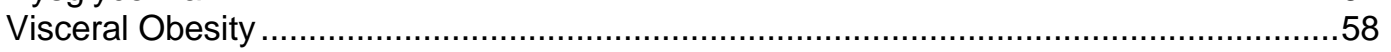

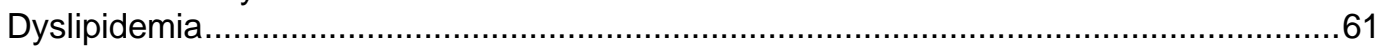

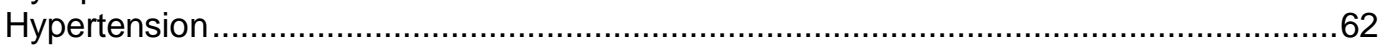

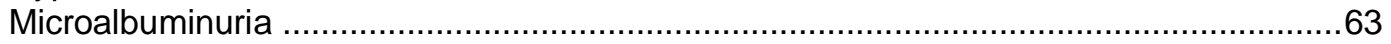


Cardiometabolic Syndrome Descriptive Epidemiology …………......................... 64

Risk Factors, Potential Covariates and Effect Modifiers .................................................66

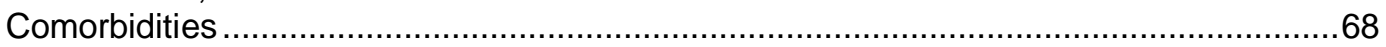

Summary

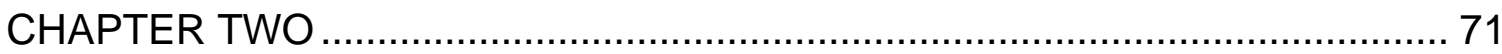

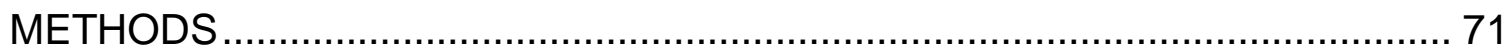

Study Design ......................................................................................... 71

Source of Study Population- National Health and Nutrition Examination Survey

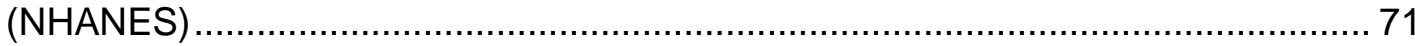

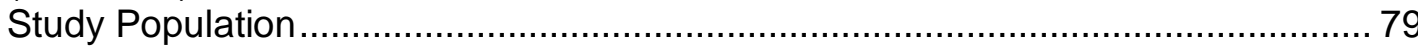

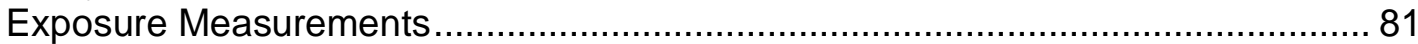

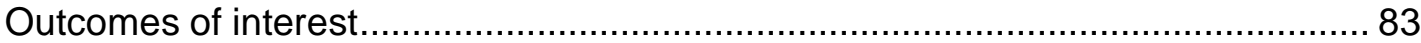

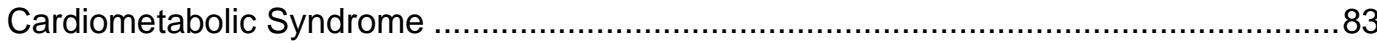

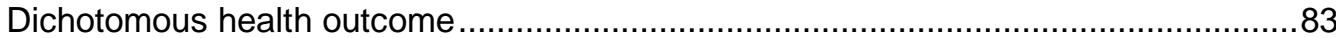

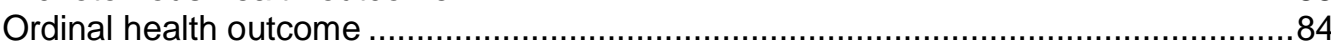

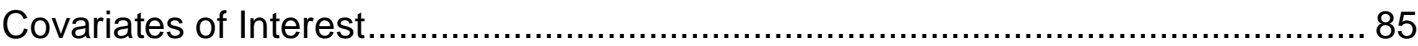

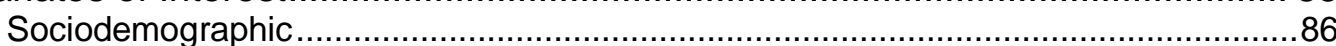

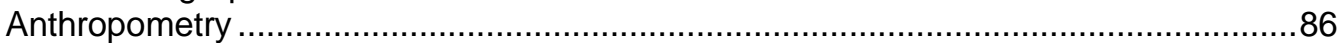

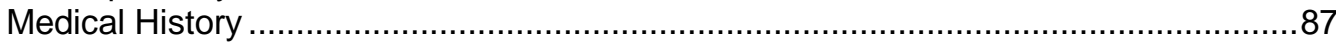

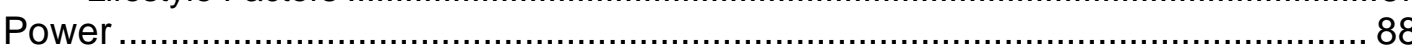

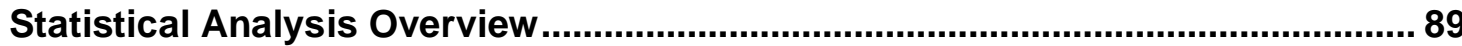

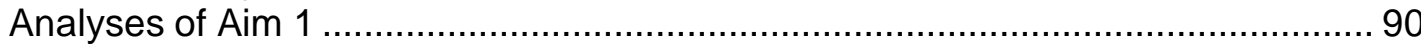

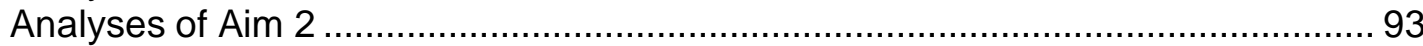

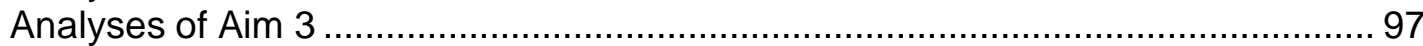

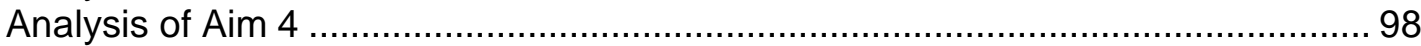

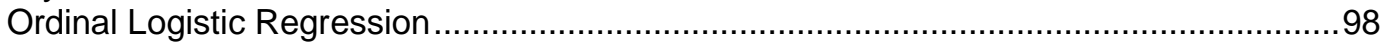

Backwards Selection ....................................................................................... 100

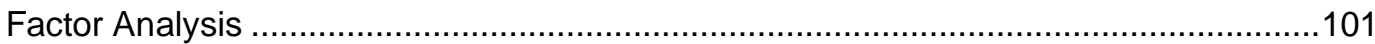

Least Absolute Shrinkage and Selection Operator (LASSO) Method...............................102

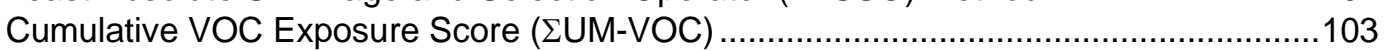

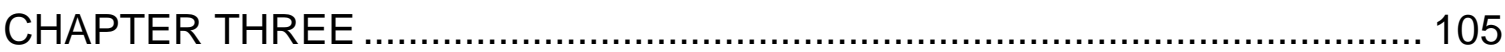

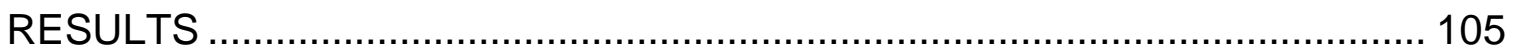

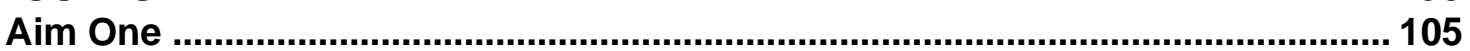

Characteristics of the Study Cohort................................................................ 105

Urinary Metabolites of Volatile Organic Compounds Characteristics .................... 109

Aim Two …................................................................................................ 117

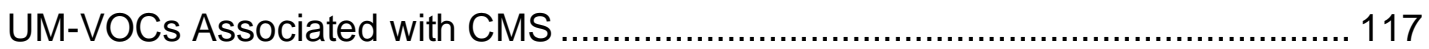

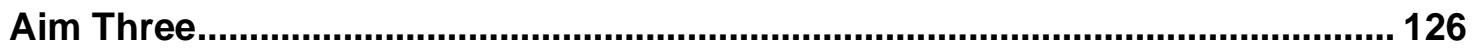

Dose-response Relationships of UM-VOCs and CMS.................................... 126

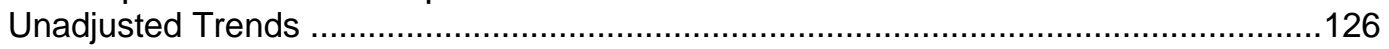

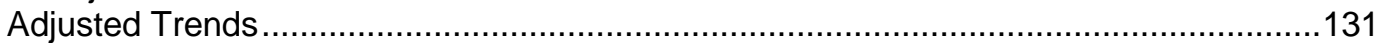

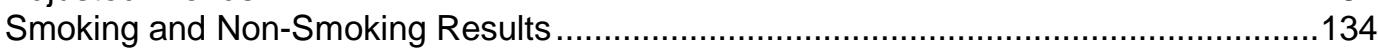

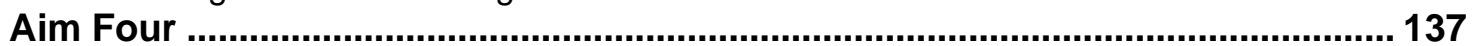

The combinatory effects of VOCs associated with CMS. ................................. 137

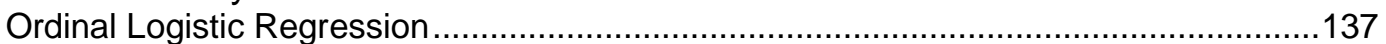

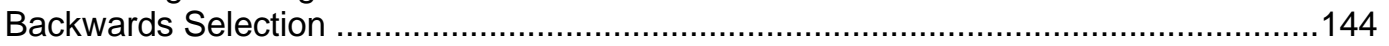

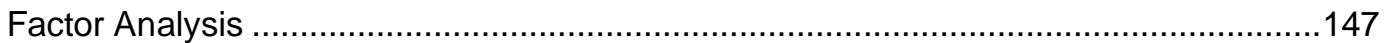

Least Absolute Shrinkage and Selection Operator (LASSO) Method.............................150

Cumulative VOC Exposure Score $(\Sigma U M-V O C)$.........................................................154 


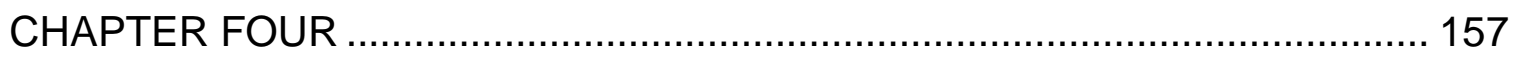

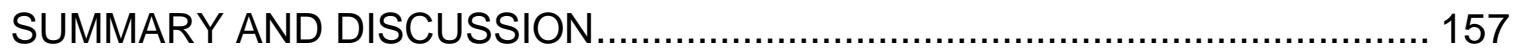

CONCLUSION

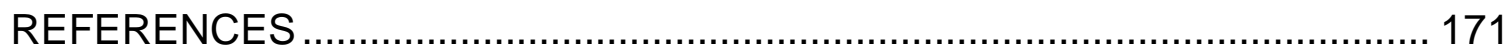

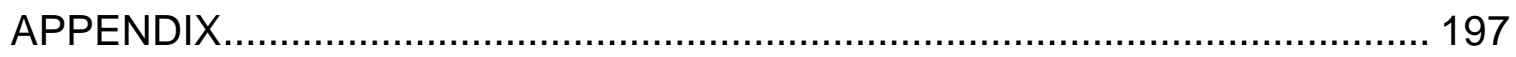

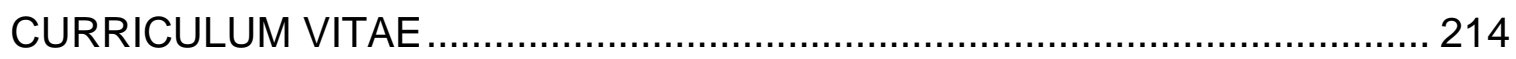




\section{LIST OF FIGURES}

PAGE

Figure 1.2. Existing Animal Information on Health Effects of Acrolein (41)........ 11

Figure 1.3. Existing Human Information on Health Effects of Acrylamide (44) ... 12 Figure 1.4. Existing Animal Information on Health Effects of Acrylamide (46) .... 12 Figure 1.5. Existing Human Information on Health Effects of Acrylonitrile (48) .. 14 Figure 1.6. Existing Animal Information on Health Effects of Acrylonitrile (51) ... 14 Figure 1.7. Existing Human Information on Health Effects of Vinyl Chloride (52)16 Figure 1.8. Existing Animal Information on Health Effects of Vinyl Chloride (54) 16 Figure 1.9. Existing Human Information on Health Effects of Ethylene Oxide (56)

Figure 1.10. Existing Animal Information on Health Effects of Ethylene Oxide (58)

Figure 1.11. Existing Human Information on Health Effects of Benzene (64) ..... 20 Figure 1.12. Existing Animal Information on Health Effects of Benzene (66) ..... 20 Figure 1.13. Existing Human Information on Health Effects of 1-Bromopropane (68) 21

Figure 1.14. Existing Animal Information on Health Effects of 1-Bromopropane (70)

Figure 1.15. Existing Human Information on Health Effects of 1,3-Butadiene (72)

Figure 1.16. Existing Animal Information on Health Effects of 1,3-Butadiene (74)

Figure 1.17. Existing Human Information on Health Effects of Carbon Disulfide

Figure 1.18. Existing Animal Information on Health Effects of Carbon Disulfide 23 (79) 25

Figure 1.19. Existing Human Information on Health Effects of Cyanide (85) ...... 29

Figure 1.20. Existing Animal Information on Health Effects of Cyanide (88) ...... 29 Figure 1.21. Existing Human Information on Health Effects of Ethylbenzene (94)

Figure 1.22. Existing Animal Information on Health Effects of Ethylbenzene (96) 33

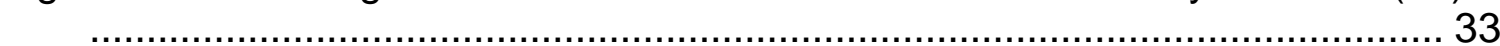

Figure 1.23. Existing Human Information on Health Effects of Styrene (101)..... 35

Figure 1.24. Existing Animal Information on Health Effects of Styrene (98) ....... 35

Figure 1.25. Existing Human Information on Health Effects of

Tetrachloroethylene (107).

Figure 1.26. Existing Animal Information on Health Effects of Tetrachloroethylene 39

Figure 1.27. Existing Human Information on Health Effects of Toluene (112) ... 41

Figure 1.28. Existing Animal Information on Health Effects of Toluene (113).... 41 
Figure 1.29. Existing Human Information on Health Effects of Trichloroethylene (118)

Figure 1.30. Existing Animal Information on Health Effects of Trichloroethylene (115) 45

Figure 1.31. Existing Human Information on Health Effects of Xylene (120) ...... 46 Figure 1.32. Existing Animal Information on Health Effects of Xylene (122)...... 47 Figure 2.1. Schematic diagram depicting inclusion/exclusion criteria for 20052006, 2011-2012, and 2013-2014 NHANES subjects used to generate the study populations for further analysis.

Figure 3.1.1. Correlations of each UM-VOC with each UM-VOC in the Full Study Cohort.

Figure 3.2.1. Forest Plot- Full Study Cohort Unadjusted Odds And 95\% Cl For 21 UM-VOCs and CMS (Dichotomous).

Figure 3.2.2. Forest Plot- Non-Tobacco Smoke Exposed Sub-Population, Unadjusted Odds And 95\% CI For 21 UM-VOCs and CMS (Dichotomous)

Figure 3.2.3. Forest Plot-Tobacco Smoke Exposed Sub-Population, Unadjusted Odds And 95\% CI For 21 UM-VOCs and CMS (Dichotomous) ................. 122

Figure 3.2.4. Forest Plot- Full Cohort, Adjusted Odds and 95\% Cl for 21 UMVOCs and CMS (Dichotomous)............................................................ 123

Figure 3.2.5. Forest Plot- Non-Tobacco Smoke Exposed Sub-Population, Adjusted Odds And 95\% CI For 21 UM-VOCs and CMS (Dichotomous).. 124

Figure 3.2.6. . Forest Plot- Tobacco Smoke Exposed Sub-Population, Adjusted Odds And 95\% CI For 21 UM-VOCs and CMS (Dichotomous) ................. 125

Figure 3.3.1. Forest Plot- Full Cohort, Unadjusted Odds and 95\% Cl for $21 \mathrm{UM}$ VOCs (quantiles) and CMS (Dichotomous) ............................................ 127

Figure 3.3.2. Full-Cohort, CMS Prevalence by UM-VOC Quintile concentration.

Figure 3.3.3. Forest Plot- Full Cohort, Adjusted Odds and 95\% Cl for 21 UMVOCs (quantiles) and CMS (Dichotomous)

Figure 3.3.4. Forest Plot- Non-Smoke Exposed: Adjusted Odds and $95 \% \mathrm{Cl}$ for 21 UM-VOCs (quantiles) and CMS (Dichotomous)

Figure 3.3.5. Forest Plot- Smoke Exposed, Adjusted Odds and 95\% Cl for 21 UM-VOCs (quantiles) and CMS (Dichotomous) .................................... 136

Figure 3.4.1. Forest Plot- Full cohort, Unadjusted ordinal odds and $95 \% \mathrm{Cl}$ for 21 UM-VOCs and cardiometabolic syndrome risk score (CMS-RS)............. 139

Figure 3.4.2. Forest Plot- Full cohort, Adjusted ordinal odds and $95 \% \mathrm{Cl}$ for 21 UM-VOCs and cardiometabolic syndrome risk score (CMS-RS). 140

Figure 3.4.3. Forest Plot- Tobacco Smoke Exposed, Adjusted ordinal odds and $95 \% \mathrm{Cl}$ for $21 \mathrm{UM}-\mathrm{VOCs}$ and cardiometabolic syndrome risk score (CMS$\mathrm{RS})$.

Figure 3.4.4. Forest Plot- Non-tobacco Smoke Exposed, Adjusted ordinal odds and $95 \% \mathrm{Cl}$ for $21 \mathrm{UM}-\mathrm{VOCs}$ and cardiometabolic syndrome risk score (CMS-RS).

Figure 3.4.5. Factor analysis path diagram of NHANES urinary metabolites of volatile organic compounds. 
Figure 3.4.6. Training set and validation set errors plots for the generalized linear model employing the LASSO fit for 21 UM-VOCs in the full cohort.

Figure 3.4.7 Coefficient Progression Plot- Standardized coefficients estimates as a function of the validations tuning parameter values for the generalized linear model employing the LASSO fit for 21 UM-VOCs in the full cohort. 152

Figure 3.4.8. Training set and validation set errors for the generalized linear model employing the LASSO fit for 21 UM-VOCs and all two-way interactions in the full cohort

Figure 3.4.9 Coefficient Progression Plot- Standardized coefficients estimates as a function of the validations tuning parameter values for the generalized linear model employing the LASSO fit for 21 UM-VOCs and all two-way interactions in the full cohort.

Figure 3.4.10. Box plot of $\Sigma U M-V O C$ exposure scores for the non-tobacco smoke exposed study cohort stratified by increasing number of components of CMS.

Figure A.1. Correlations of each UM-VOC with each UM-VOC- non-tobacco smoke exposed cohort.

201

Figure A.2. Correlations of each UM-VOC with each UM-VOC- tobacco smoke exposed cohort 202 


\section{LIST OF TABLES}

PAGE

Table 1.1. Compilation of Existing Information on Health Effects of the VOCs .. 48 Table 1.2. World Health Organization Definition of Cardiometabolic Syndrome 56

Table 1.3. World Health Organization Cut-Points and Risk of Metabolic Complications $(182,184)$ 61

Table 2.1. Parent Volatile Organic Compounds and Metabolites of Interest ...... 82

Table 2.2. Cardiometabolic Syndrome Risk Factor Variables and Definitions.... 84

Table 2.3. Cardiometabolic Syndrome Risk Score. 85

Table 2.4. Non-Survey Weighted Minimal Detectable Difference Calculation Data

Table 3.1.1. Study Population Characteristics Stratified by NHANES Survey Cycle.......................................................................................... 106

Table 3.1.2. Study Population Characteristics Stratified by Cardiometabolic

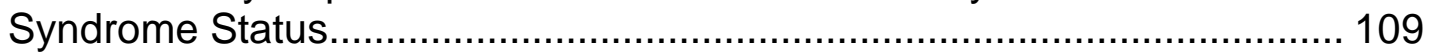

Table 3.1.3. Volatile Organic Compound Metabolite Level of Detection ........... 111

Table 3.1.4. Description of Volatile Organic Compound Metabolites for Appended NHANES Study Population.

Table 3.1.5. Description of Volatile Organic Compound Metabolites Stratified by Cardiometabolic Syndrome Status. 114

Table 3.1.6 Description of Volatile Organic Compound Metabolites with Significant Differences Based on CMS Status Stratified by Tobacco Smoke Exposure

Table 3.4.1. Identification of UM-VOCs included in backward selection analysis.

Table 3.4.2. Analysis of maximum likelihood estimates....................................... 147

for the final logistic regression model produced by the .................................. 147

backwards selection method....................................................................... 147

Table 3.4.3 mean $\Sigma$ UM-VOC exposure score for all adjustment variables in the $\Sigma$ UM-VOC exposure score logistic regression models for non-tobacco smoke exposed participants. 155

Table A.1. Study Population with Cardiometabolic Syndrome Characteristics Stratified by Gender. 197

Table A.2. Volatile Organic Compound Metabolite Level of Detection Stratified by Tobacco Smoke Exposure Status. 198

Table A.3. Description of Volatile Organic Compound Metabolites by NHANES Cycle

Table A.4. Full Appended Study Group UM-VOC Correlation Matrix............... 200 
Table A.5. Unadjusted and adjusted odds of prevalent cardiometabolic disease associated with 21 urinary metabolites of volatile organic compound concentrations in NHANES participants 2005-06, 2011-12, and 2013-14.

Table A.6. Unadjusted tobacco smoke exposed and non-tobacco smoke exposed odds of prevalent cardiometabolic disease associated with 21 urinary metabolites of volatile organic compound concentrations in NHANES participants 2005-06, 2011-12, and 2013-14.

Table A.7. Adjusted tobacco smoke exposed and non-tobacco smoke exposed odds of prevalent cardiometabolic disease associated with 21 urinary metabolites of volatile organic compound concentrations in NHANES participants 2005-06, 2011-12, and 2013-14.

Table A.8. Full Cohort, Unadjusted odds of prevalent cardiometabolic syndrome (dichotomous) associated with 21 urinary metabolites of volatile organic compound concentrations (quintiles) in NHANES participants 2005-06, 2011-12, and 2013-14

Table A.9. Full Cohort, Adjusted odds of cardiometabolic syndrome (dichotomous) associated with 21 urinary metabolites of volatile organic compound concentrations (quintiles) in NHANES participants 2005-06, 2011-12, and 2013-14

Table A.10. Non-tobacco smoke exposed, Adjusted odds of prevalent cardiometabolic syndrome associated with 21 urinary metabolites of volatile organic compound concentrations in NHANES participants 2005-06, 201112 , and 2013-14.

Table A.11.Tobacco smoke exposed, Adjusted odds of prevalent cardiometabolic disease associated with 21 urinary metabolites of volatile organic compound concentrations in NHANES participants 2005-06, 2011-12, and 2013-14.

Table A.12. Full cohort, Unadjusted odds of ordinal cardiometabolic syndrome risk score associated with 21 urinary metabolites of volatile organic compound concentrations in NHANES participants 2005-06, 2011-12, and 2013-14

Table A.13. Adjusted odds of ordinal cardiometabolic syndrome risk score associated with 21 urinary metabolites of volatile organic compound concentrations in NHANES participants 2005-06, 2011-12, and 2013-14.

Table A.14. Tobacco Smoke Exposed- Adjusted odds of ordinal cardiometabolic syndrome risk score associated with 21 urinary metabolites of volatile organic compound concentrations in NHANES participants 2005-06, 201112 , and 2013-14.

Table A.15. Non-Tobacco Smoke Exposed- Adjusted odds of ordinal cardiometabolic syndrome risk score associated with 21 urinary metabolites of volatile organic compound concentrations in NHANES participants 200506, 2011-12, and 2013-14 


\section{CHAPTER ONE}

\section{INTRODUCTION}

\section{Rationale and significance of the study}

Cardiometabolic syndrome (CMS) describes a spectrum of dysfunctional cardiovascular and metabolic system functions (1). CMS begins with insulin resistance, often leading to metabolic syndrome and pre-diabetes and finally progresses to more sever conditions including cardiovascular disease and type II diabetes mellitus (T2DM) (1). These conditions are grouped together under the umbrella terms "cardiometabolic syndrome" or "cardiometabolic disease" due the shared risk factors among the conditions; risk factors such as overweight and obesity, dyslipidemia, and high blood pressure (1).

The consequences of CMS are severe. Cardiovascular disease is the leading cause of death, both globally and in the United States (U.S.) and type II diabetes has grown to epidemic proportions (2-4). Cardiovascular disease is responsible for $30 \%$ of all death in the U.S. and it has been estimated that by 2030 the rate of diabetes in the U.S. will have risen from 17.7 million in 2000 to nearly 30.3 million $(3,5)$. Considering the associated morbidities, decreased quality of life, high social costs, and economic costs (U.S.: 80 billion dollars annually), CMS is among the leading priorities of our time. 
It is well established that suboptimal diet and physical inactivity are the two most important modifiable risk factors for the development of CMS $(6,7)$. Still, understanding all contributing factors in the development of CMS, particularly modifiable risk factors such as environmental air pollution exposures, is needed.

Environmental air pollution is the leading environmental risk factor that contributes to the global burden of disease (8). 6.5 million deaths were attributed to air pollution exposure in 2012 (9); more recent estimates indicate that more than 9 million deaths per year may be attributable to air pollution exposure (8). This upward trend in deaths attributable to air pollution exposure suggests that the contribution of air pollution to global mortality may be increasing (10).

Volatile Organic Compounds (VOCs) are one of a number of constituents of air pollution. VOCs directly contribute to the formation of secondary organic aerosols, a major component of fine PM $(\leq 2.5 \mu \mathrm{m}$ in aerodynamic diameter; PM2.5), and tropospheric ozone $\left(\mathrm{O}_{3}\right)(11-13)$. $\mathrm{PM}_{2.5}$ levels in ambient air have been shown in previous epidemiologic cohort studies to be significantly associated with all-cause and cardiorespiratory mortality as well cardiovascular disease risk and progression $(8,14,15)$. Such associations have also been observed even at concentrations of $\mathrm{PM}_{2.5}$ and $\mathrm{O}_{3}$, that are below the current U.S. standards (16). A number of studies have also indicated that adverse health effects of PM vary with the composition of the polluted air (17-21).

Although most previous work has found that PM2.5 levels are associated with adverse health outcomes, polluted air contains a variety of gaseous copollutants such as ozone and nitrous oxides. Additionally, polluted ambient air, 
particularly in urban locations, contains a wide range of VOCs, such as benzene, acrolein, xylene, and formaldehyde (22). It has been shown widely in the literature, both in animal exposure models and human studies, that exposure to VOCs is associated with systemic, immunologic, neurologic, reproductive, developmental, genotoxic, and carcinogenic effects (23). Furthermore, several of the VOCs of interest have been placed on the federal Substance Priority List as being among the chemicals of top public health concern (23).

\section{Specific Aims of the study}

Current evidence for the direct effects of VOCs on cardiovascular and metabolic outcomes is not robust; however, there are strong implications that environmental pollutants such as VOCs play a role in cardiometabolic syndrome. CMS is defined by the World Health Organization (WHO) as the presence of dysglycemia, plus two or more of the following criteria: abdominal obesity, high triglycerides, low-high density lipoproteins, hypertension, and/or the presence of microalbuminuria $(14,24)$. The available epidemiologic and experimental data suggest that exposure to VOCs contributes to the cause of cardiovascular disease, hypertension, and metabolic dysfunction, but further investigation is needed.

Furthermore, it is well established by both epidemiological and toxicological studies that particulate matter (PM), a constituent of air pollution, is significantly associated with CMS (14). One method for assessing the health effects of PM, is

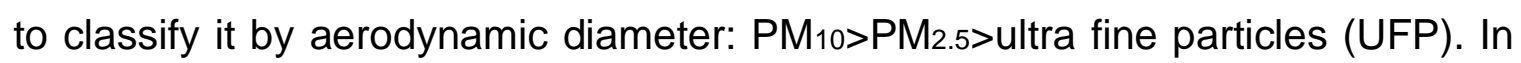
brief, the smaller the particle, the more hazardous it is to health, as the smaller particles can travel more deeply into the lungs (14). VOCs contribute to the 
composition, as well as to the mass of PM, but it is not known if particle mass or the chemical properties of constituent VOCs or a combination of the two is the most harmful to cardiometabolic health. Until the relative importance of VOCs' contribution to particle mass and their chemical properties is determined and until the most harmful compounds are identified, the only practical approach to plausibly reducing adverse health effects would be to reduce overall emissions of VOCs. Therefore, information regarding the links between VOC and CMS would strongly reinforce priorities in public health policy and planning and incentives for pursuing specific strategies to change unhealthy environmental contributions and ultimately reduce cardiometabolic health-related burdens. An investigation of the associations between VOCs with CMS will lend insight into the contribution of one of the major modifiable constituents of PM and its role in CMS.

The aim of this study is to assess whether exposure to VOCs is associated with CMS. Therefore, national cross-sectional associations between personallevel exposures to VOCs and the prevalence of CMS will be examined. For this analysis, data will be obtained on individual-level exposure and disease status from participants of the National Health and Nutrition Examination Survey (NHANES). Individual-level exposure will be estimated by the concentration of the urinary metabolites of VOCs (UM-VOCs), which correspond to individual-level exposures to VOCs (25).

We hypothesize that increasing levels of UM-VOCs will be positively associated with an increase in the prevalence of CMS. Associations with VOCs and CMS will be examined linearly and categorically and from both individual and 
multi-pollutant exposure perceptive.

These novel nationally-representative estimates of personal-level exposure to VOCs derived from individual participants' urinary metabolites of VOCs provide unique insight into associations and patterns of personal exposure to VOCs and their associations with CMS in the United States. To date, quantitative and nationally representative associations of individual-level exposure to VOCs using urinary biomarkers of exposure and their associations with CMS have not been reported.

To address these gaps in knowledge, the objective of the analysis is to evaluate the association between VOC exposures and CMS. The central hypothesis for this dissertation is that cardiometabolic syndrome (CMS) is positively associated with increasing concentrations of urinary metabolites of volatile organic compounds (UM-VOCs).

The hypothesis will be tested with the following specific aims:

Specific Aim 1: Statistically describe and compare distributions, trends, and cohort characteristics of the 2005-2006, 2011-2012, and 2013-2014 NHANES UMVOC sub-sample.

Specific Aim 2: Study the effects of individual continuous UM-VOCs and prevalent CMS after adjusting for select covariates.

Specific Aim 3: Evaluate the dose-response relationships of individual categorical UM-VOC exposures and CMS.

Specific Aim 4: Explore combinatory effects of VOCs on CMS. 


\section{Literature Review}

\section{Volatile Organic Compounds}

Volatile organic compounds (VOCs) are numerous and ubiquitous in the environment. VOCs are a class of organic compounds whose molecules contain carbon (26). VOCs are generally considered any organic compound with and initial boiling point of less than $250^{\circ} \mathrm{C}\left(482^{\circ} \mathrm{F}\right)$ measured at standard atmospheric pressure (101.3Kpa) (27). VOCs are naturally occurring as well man-made chemicals, and are volatile by nature because of their low intermolecular attractive binding forces (27).

VOCs have high vapor pressures, due to their low boiling points, which leads to the high tendency for these chemicals to vaporize, which is known as volatility. Volatile organic compounds exist in the vapor phase, particle phase, or both, depending on the situational equilibrium properties (e.g., vapor pressure) and particle surface composition (e.g., water content) (28). Since the volatility of a compound mostly increases as temperature increases and the boiling point of VOCs is relatively low, the state of the compound, whether vapor or particle, is dependent on temperature. It has been estimated that for every one degree increase in temperature there is a $1.3 \pm 0.4 \%$ increase in daily total motor vehicle VOC emissions (28). In addition to the effects of temperature on volatility, exposure to sunlight also increases chemical reaction rates (29). Temperature and sunlight are important consideration, as their presence or lack thereof, impact secondary pollutant formation. 
Volatile organic compounds are released directly into the air, or by incomplete combustion in the burning of organic matter. Volatile organic compounds are of concern as both indoor and outdoor air pollutants, and while industrial emission sources of VOCs in the U.S. have been identified and are regulated, their extensive use in industry and transportation make them quite abundant in the environment and therefore, currently exposure to anthropogenic VOCs is virtually unavoidable $(30,31)$. Common sources of exposure to anthropogenic VOCs are industrial emissions, tobacco smoke and automobile exhaust, additionally common house hold sources of exposure to VOCs include paints, pesticides, deodorizers, cleaning and degreasing agents, personal care products, and solvents (30-33).

In addition to anthropogenic sources of VOCs there are significant natural sources of VOCs. In fact, in the United States, it has been estimated that biogenic sources of VOCs account for approximately $74 \%$ of VOC emissions and globally as approximately $90 \%$ of VOC emissions $(34,35)$. While biogenic sources comprise the vast majority of $\mathrm{VOC}$ emissions, anthropogenic sources are of particular interest for a number of reasons. First anthropogenic VOCs, in contrast with biogenic VOCs, are extremely concentrated exposures (36). In fact anthropogenic emissions of VOCs (automobile exhaust, vapors from fueling vehicles, solvents from paints, etc.) are largely concentrated in urban areas, which cover only approximately three percent of the Earth's surface (37). This means, that approximately $10 \%$ of the total VOC emissions produced by humans are highly concentrated where most of the human population lives. So in urban areas, 
anthropogenic $\mathrm{VOC}$ emissions are about 4 times stronger than the average background of natural emissions (36). These highly concentrated VOC emissions occur where most of the human population lives and thus have a much larger effect on human exposure than do natural emissions (36). Additionally many of these highly concentrated VOCs have been associated with adverse health effects (34). Anthropogenic VOCs may tip a delicate natural balance causing pollutants to interact in ways they otherwise would not. Lastly, anthropogenic sources of VOCs are of particular interest because they can be decreased through regulatory and voluntary actions, leading to improvements in air quality (34).

Several agencies and countries have varying definitions of VOCs. The Canadian definition is focused on commonly encountered VOCs that affect air quality such as benzene, toluene, and methane (38). The U.S. Environmental Protection Agency's (EPA) definition is focused on reactive chemicals, which create photochemical smog. The U.S. EPA definition states, "volatile organic compounds (VOC) means any compound of carbon, excluding carbon monoxide, carbon dioxide, carbonic acid, metallic carbides or carbonates and ammonium carbonate, which participates in atmospheric photochemical reactions, except those designated by EPA as having negligible photochemical reactivity" (39).

There is an abundance of VOCs in the environment from both natural and man-made sources. For the purpose of this review, volatile organic compounds with measurable urinary metabolites will be assessed. Namely the metabolites of the parent VOCs of interest are: acrolein, acrylamide, acrylonitrile, vinyl chloride, ethylene oxide, benzene, 1-bromopropane, 1,3-butadiene, crotonaldehyde, N, N- 
dimethylformamide, ethylbenzene, styrene, propylene oxide, tetrachloroethylene, toluene, trichloroethylene, and xylene.

Health Effects of Volatile Organic Compounds

\section{Individual VOC Effects}

Nearly 200 air toxics have been associated with adverse health effects in occupational, epidemiologic, and/or laboratory studies. The 19 parent VOCs of interest in this investigation have all been associated with adverse health effects. The majority of the literature on the health effects of VOC exposures is related to the carcinogenicity of individual VOC exposures. The descriptions below include a brief overview of the physical description, common sources, common routes of exposure, and health effects of each parent VOC of interest in this investigation. The tables included for each of the 19 parent VOCs in the following summary descriptions provide important information on reported health effects for the given route of exposure for both human and animal exposures (40).

\section{Acrolein}

Acrolein is a colorless or yellow liquid which has an acrid smell (41). Acrolein is used industrially as an intermediate in chemical synthesis and as a biocide. It is generated in the breakdown or burning of lipids and organic matter (42). Acrolein is also a natural byproduct of many pollutants found in outdoor air, and is produced in the burning of tobacco and other plant material including coal and gasoline. Acrolein exposures can occur via industrially contaminated air and water. Additionally, because acrolein is produced when fats are overheated, small 
amounts of acrolein are can be found in food (41).

Due to inadequate evidence, acrolein has not been classified as a carcinogen by the Department of Health and Human Services (DHHS), International Agency for Research on Cancer (IARC), or the U.S. Environmental Protection Agency (U.S. EPA) (41). However, acrolein is capable of inducing sister chromatid exchange, DNA cross-linking, and mutations under certain conditions. Additionally, acrolein has been established as a cardiovascular, hematological, ocular, and respiratory irritant and disrupter (41). Acute high dose exposures may cause death while acute low dose exposures may cause irritation to the nasal cavity, lowered breathing rate, damage to the lining of the lungs, stomach irritation, vomiting, stomach ulcers and bleeding. The effects of chronic moderate acrolein exposure are not well established (41). Figures 1.1 and 1.2 below provide a summary of existing information on the health effects of acrolein (41).

\begin{tabular}{|c|c|c|c|c|c|c|c|c|c|c|}
\hline & \multirow[b]{2}{*}{ 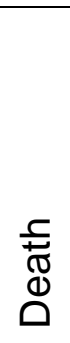 } & \multirow[b]{2}{*}{$\frac{0}{5}$} & \multicolumn{2}{|c|}{ Systemic } & \multirow[b]{2}{*}{ 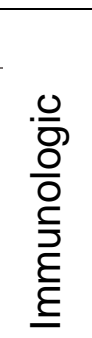 } & \multirow[b]{2}{*}{$\begin{array}{l}\frac{0}{0} \\
\frac{0}{0} \\
\frac{0}{2} \\
\frac{0}{2}\end{array}$} & \multirow[b]{2}{*}{$\begin{array}{l}\stackrel{0}{D} \\
\frac{0}{0} \\
\frac{0}{0} \\
\frac{0}{0} \\
0 \\
\simeq\end{array}$} & \multirow[b]{2}{*}{ 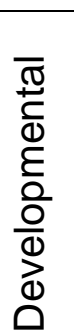 } & \multirow[b]{2}{*}{$\begin{array}{l}\frac{0}{X} \\
0 \\
\frac{0}{0} \\
\frac{L}{0} \\
0\end{array}$} & \multirow[b]{2}{*}{ 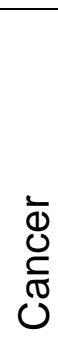 } \\
\hline & & & 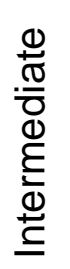 & $\begin{array}{l}\frac{0}{\bar{L}} \\
\frac{\partial}{\bar{D}} \\
\end{array}$ & & & & & & \\
\hline Inhalation & & - & & & & & & & & \\
\hline Oral & & & & & & & & & & \\
\hline Dermal & & - & & & & & & & & \\
\hline
\end{tabular}

Figure 1.1. Existing Human Information on Health Effects of Acrolein (41) 


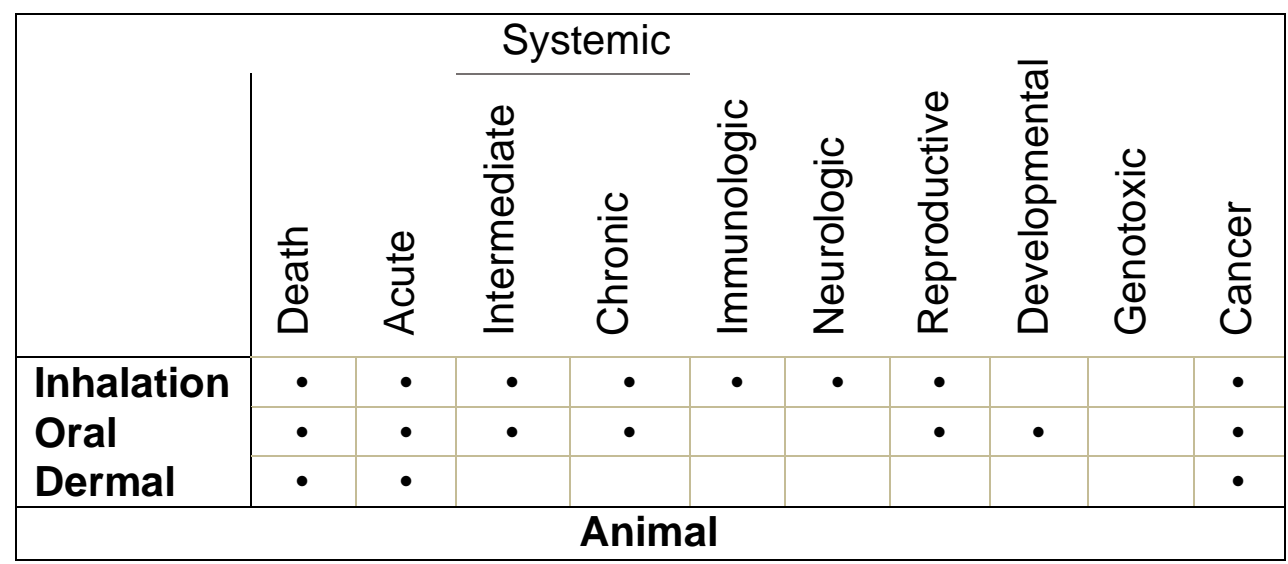

Figure 1.2. Existing Animal Information on Health Effects of Acrolein (41)

\section{Acrylamide}

Acrylamide is a white/colorless, odorless, crystalline chemical that is highly soluble in water (43). Acrylamide is used industrially as a reagent in the synthesis of polymers, pulp and paper, and textiles (43). Acrylamide is generated in the heating of starch-rich materials (43).

Acrylamide exposures in humans generally occur through contact with contaminated water and food. It has been reported that consumption of starch-rich foods is the primary exposure source to the non-smoke exposed general public (44). Smoking and exposure to highly polluted air are the greatest sources of acrylamide in the non-occupationally exposed general public (44).

Acrylamide is considered to be a potential carcinogen and is classified as a group 2A carcinogen (probably carcinogenic to humans) by the International Agency for Research in Cancer (IARC) (44). Exposure limits have been set by both the Occupational Safety and Health Administration (OSHA) and the National Institute for Occupational Safety and Health (NIOSH) (44). These exposure limits are set 
to protect the national work force and are considered to be the upper limit of the acceptable concentration of a hazardous substance in workplace air (45). Figures 1.3 and 1.4 provide a summary of existing information on the health effects of acrylamide (44).

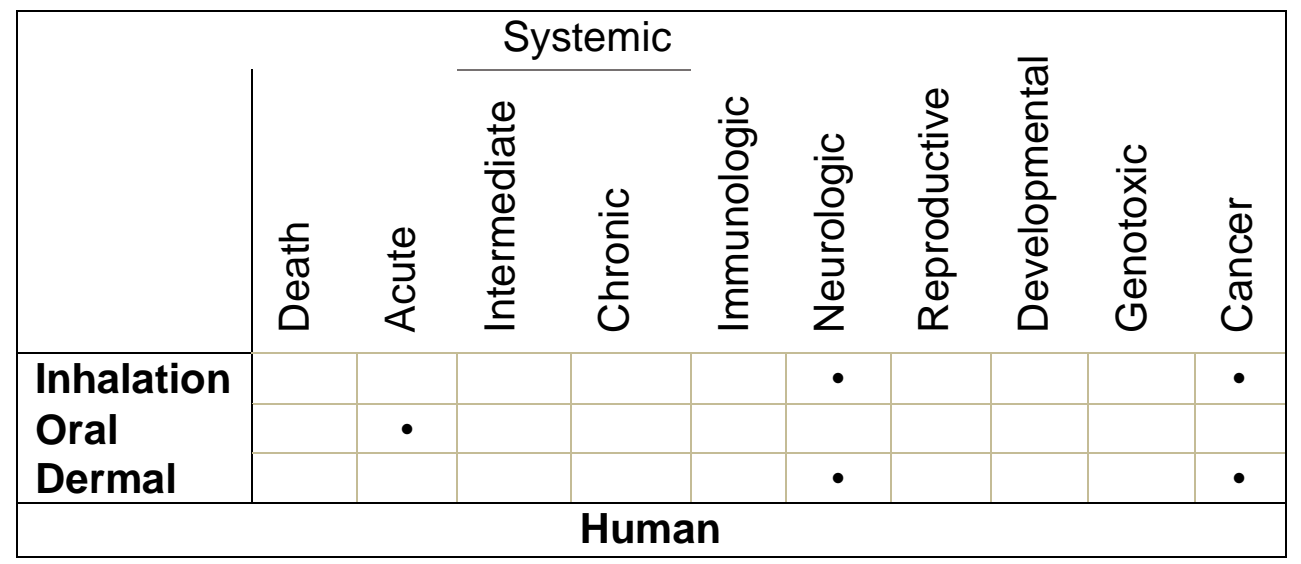

Figure 1.3. Existing Human Information on Health Effects of Acrylamide (44)

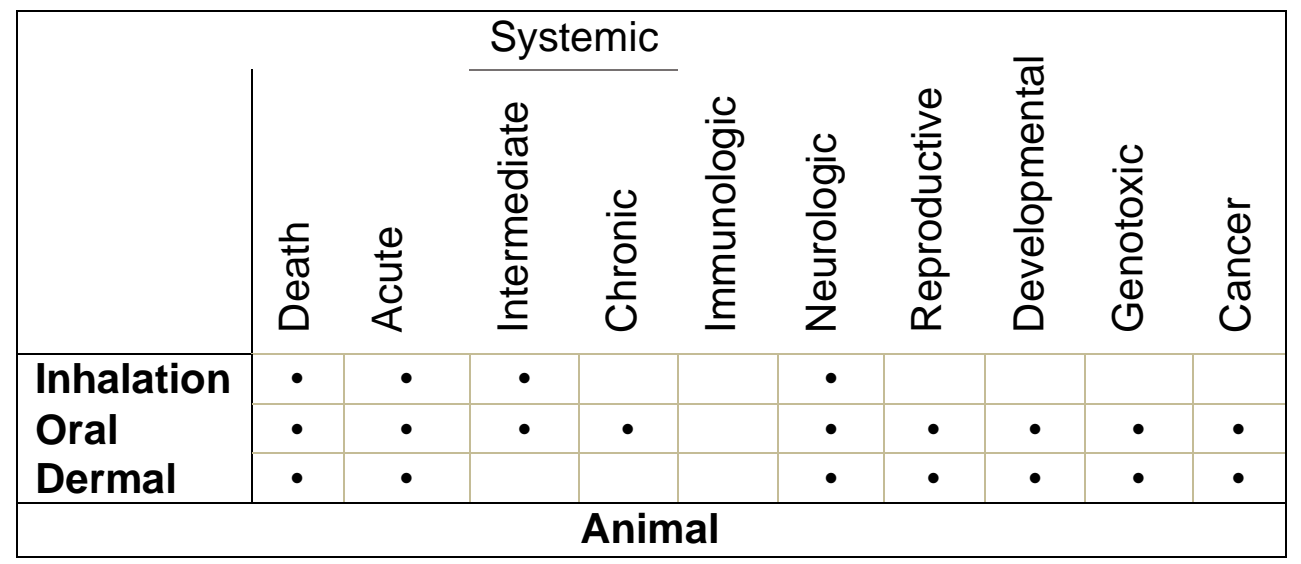

Figure 1.4. Existing Animal Information on Health Effects of Acrylamide (46)

\section{Acrylonitrile}

Acrylonitrile is a colorless, highly flammable liquid with a pungent onion-like smell. Acrylonitrile is highly soluble in water (47). Acrylonitrile is used industrially to produce rubber, resins, plastics, elastomers, synthetic fiber, pharmaceuticals, antioxidants, and dyes, and in the manufacture of carbon fiber (47). Previously, 
acrylonitrile was used as a pesticide fumigant but all pesticide uses have been discontinued.

Acrylonitrile exposure is generally associated with occupational exposures but pathways of exposure to the general public include industrial releases, automobile exhaust, food packaging, and cigarette smoke (48). Exposures associated with contaminated air, water, and soil from locations in close proximity to acrylonitrile factories or hazardous waste sites have also been reported (48).

Acute high dose exposures of acrylonitrile generally effect the nervous system and have been reported to cause headaches, nausea, disorientation and death (48). Other reported effects from high dose exposures to acrylonitrile include damage to red blood cells and liver, skin blistering and peeling, increased number of tumors occurring in the brain, salivary glands, and intestines and increased incidence of lung cancer $(48,49)$. Additionally, reproductive effects and birth defects have also been observed in animals (48).

Acrylonitrile is considered reasonably anticipated to be a human carcinogen by the Agency for Toxic Substances and Disease Registry (ATSDR) and is classified by the U.S. Environmental Protection Agency (U.S. EPA) as a B1; probable human carcinogen, and as a group B2; possibly carcinogenic to humans, by the International Agency for Research on Cancer (IARC) (48-50). Figures 1.5 and 1.6 provide a summary of existing information on the health effects of acrylonitrile (48). 


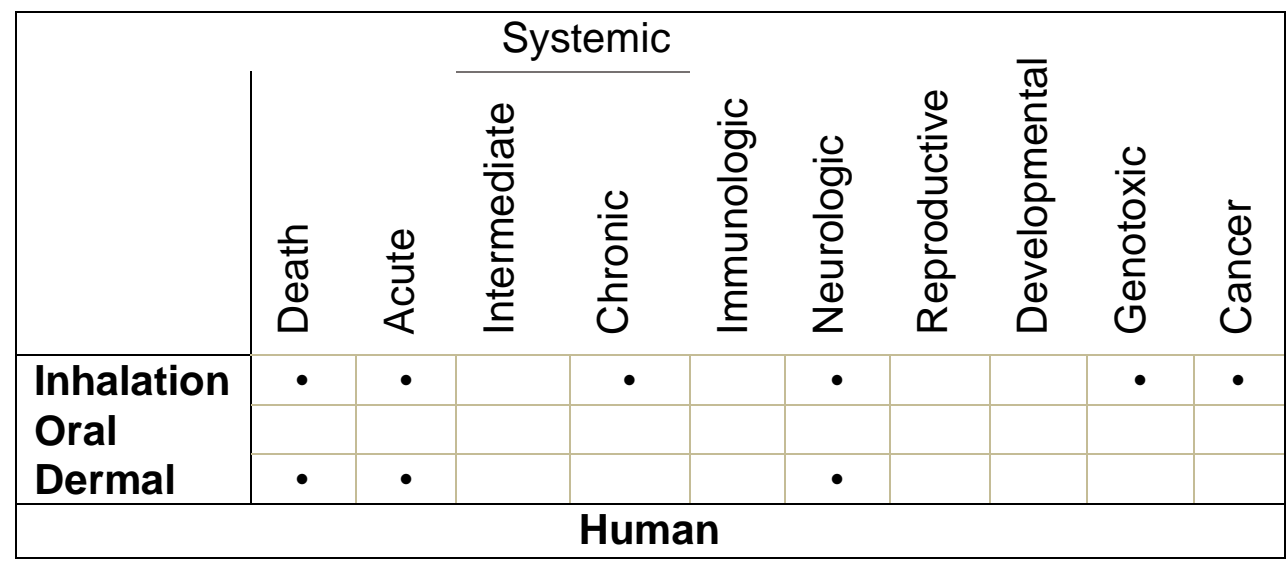

Figure 1.5. Existing Human Information on Health Effects of Acrylonitrile (48)

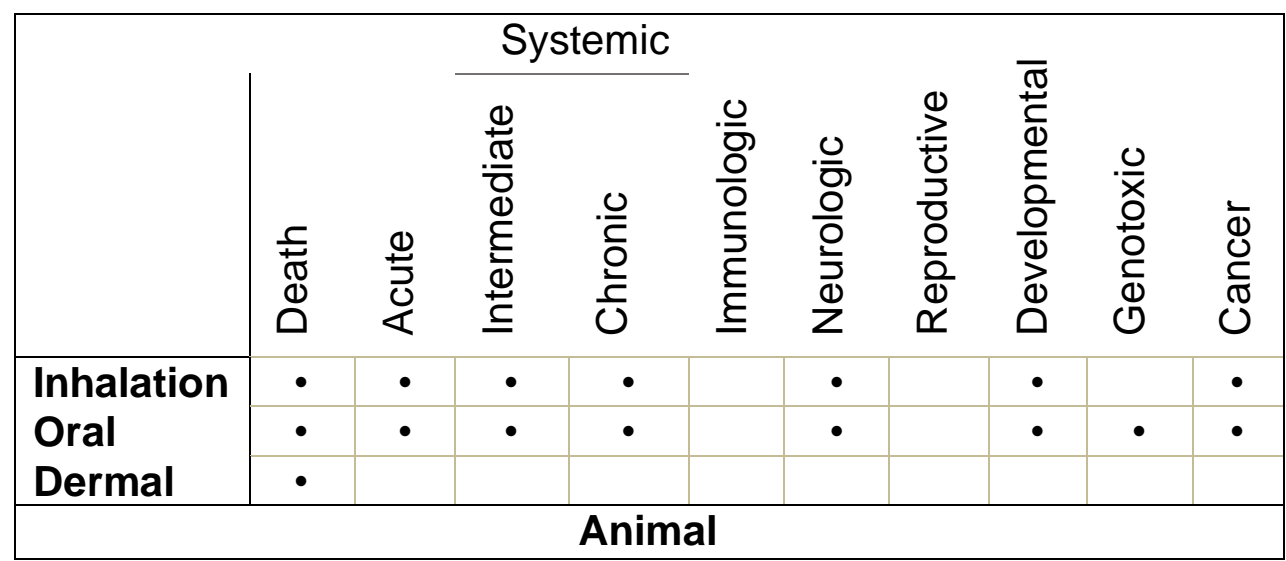

Figure 1.6. Existing Animal Information on Health Effects of Acrylonitrile (51)

\section{Vinyl Chloride}

Vinyl chloride is a flammable colorless gas, with a mild sweet odor (52). Vinyl chloride is a manmade chemical chiefly used in the production of the polymer polyvinyl chloride (PVC). Vinyl chloride is one of the top 20 largest petrochemicals in production worldwide and is used to make a variety of plastic products including pipes, wire and cable coatings, packaging materials, upholstery, house ware, automobile parts, etc. (52). Vinyl chloride was previously used as an aerosol propellant, a coolant, and in some cosmetics (52). 
Vinyl chloride is commonly found in the air near vinyl chloride manufacturing and processing plants, hazardous waste sites, and landfills and is also present in tobacco smoke. In addition to air exposures, individuals can also be exposed to vinyl chloride though contaminated water sources (52).

Acute high dose exposures of vinyl chloride affect the central and peripheral nervous system casing dizziness, drowsiness, unconsciousness, headache and death. Animal studies have shown extremely high levels exposures of vinyl chloride can cause liver, kidney, lung, and heart damage and prevent blood clotting. Chronic exposure to vinyl chloride causes a set of symptoms that is characterized by Raynaud's phenomenon, joint and muscle pain and sclerodermalike skin changes. Other symptoms include eye, nose, throat, and skin irritations, weight loss, and abdominal pain. Vinyl Chloride exposure is associated with developing liver, brain and lung cancer as well as cancer of the lymphatic and hematopoietic system (53).

Vinyl chloride is considered to be a known human carcinogen with sufficient evidence in both humans and animals. This distinction is also recognized by IARC, the U.S. EPA, ATSDR and several other agencies (50, 52, 53). Figures 1.7 and 1.8 provide a summary of existing information on the health effects of vinyl chloride (52). 


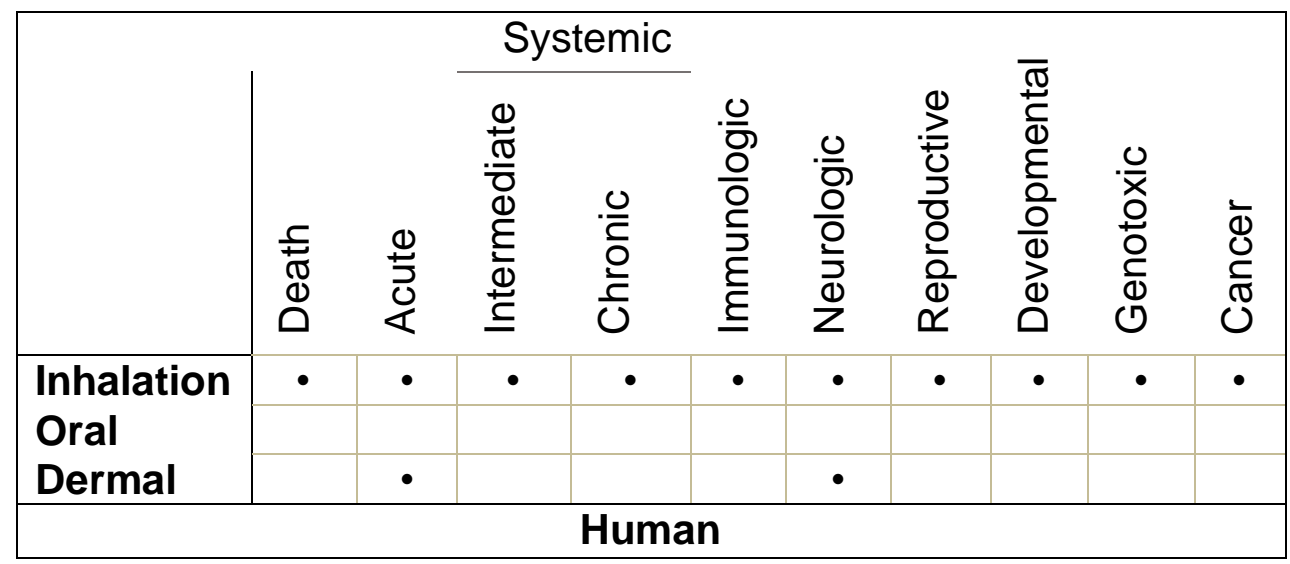

Figure 1.7. Existing Human Information on Health Effects of Vinyl Chloride (52)

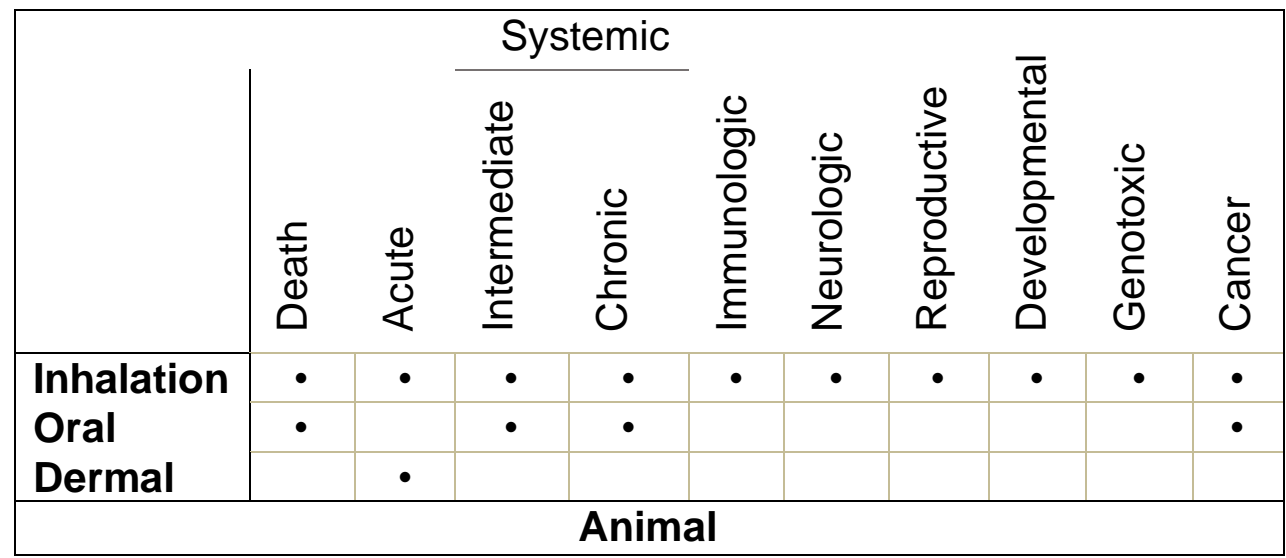

Figure 1.8. Existing Animal Information on Health Effects of Vinyl Chloride (54)

\section{Ethylene Oxide}

Ethylene oxide, also called oxirane by the International Union of Pure and Applied Chemistry (IUPAC), is a flammable, colorless, faintly sweet smelling gas (55). Ethylene oxide dissolves easily in water, alcohol, and most organic solvents. Ethylene oxide is widely used as a chemical intermediate in industry to make detergents, thickeners, solvents, plastics, as a sterilizing agent for medical supplies and food, as an insecticide fumigant, and as a bactericidal, fungicidal, and sporicidal disinfectant (55). 
Ethylene oxide is not widely found in the general environment and due to lack of information it is not known if exposures of ethylene oxide are common from contaminated air, water, or soil near hazardous waste sites. Most ethylene oxide exposures are occupational exposures of medical staff or agricultural workers (56). Although ethylene oxide is commonly used on food crops it is not known if this is a source of exposure to the general public (56).

Acute ethylene oxide exposures consist mainly of central nervous system effects and irritations of the eyes, nose, skin, and mucous membranes. Chronic exposures cause functional problem in the brain and nerves and have been associated with increased incidence of leukemia, stomach cancer, cancer of the pancreas, and Hodgkin's disease. There is also evidence of increased rates of miscarriage and birth defects (56).

Ethylene oxide is classified as a probable/suspected human carcinogen (Group B1) by IARC and the U.S. EPA but is classified as a known human carcinogen by ATSDR and the National Toxicology Program $(50,56,57)$. Figures 1.9 and 1.10 provide a summary of existing information on the health effects of ethylene oxide (56). 


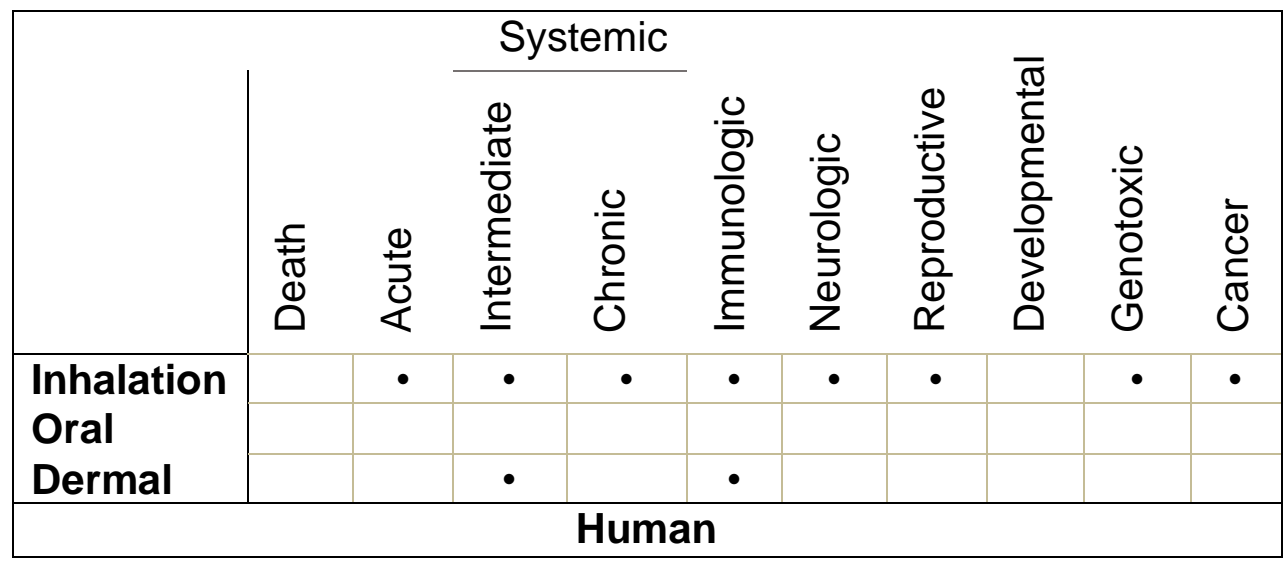

Figure 1.9. Existing Human Information on Health Effects of Ethylene Oxide (56)

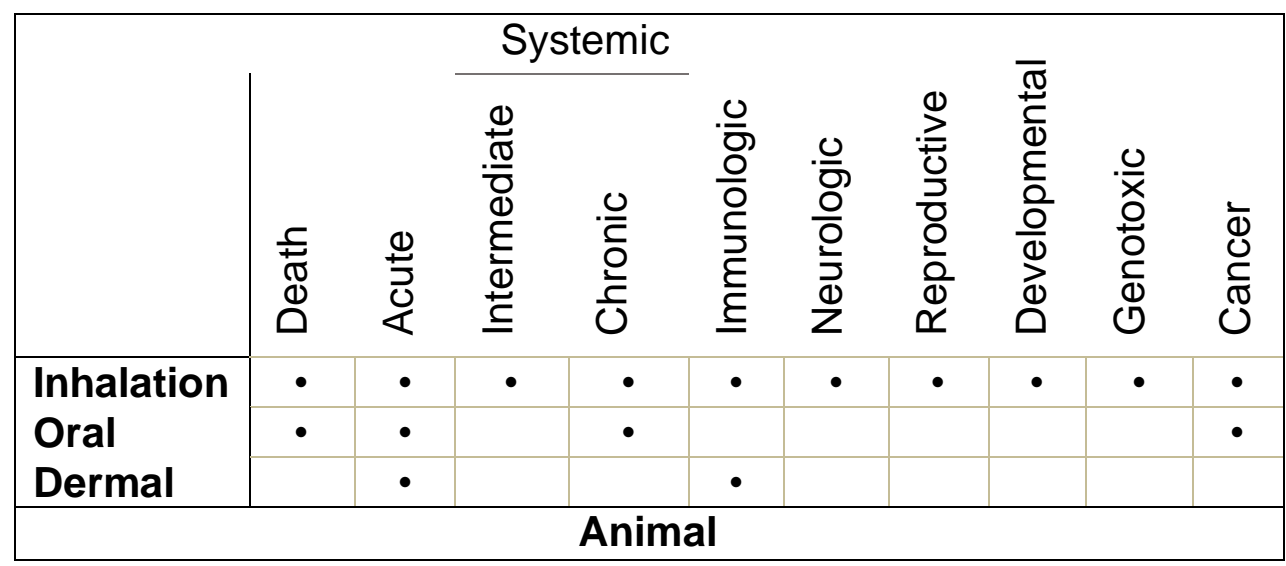

Figure 1.10. Existing Animal Information on Health Effects of Ethylene Oxide (58)

\section{Benzene}

Benzene is a colorless, highly flammable, sweet smelling aromatic liquid. Benzene is a good solvent for many organic and inorganic substances and is therefore widely used as an important industrial solvent and precursor. Benzene is one of the top twenty chemicals in productive volume and is widely used in the United States (59).

Benzene is an important industrial solvent and precursor in the production of drugs, plastics, synthetic rubber and dyes and is a natural constituent of crude 
oil (59). While industrial emission sources have been identified and are regulated, benzene's extensive use in industry and transportation make it quite abundant in the environment and therefore industrial exposure sources are virtually unavoidable (60). In addition to the abundant industrial emission exposures, the general public is exposed to benzene via numerous common-use products. Benzene is present in every-day products, such as, tobacco smoke, automobile exhaust, industrial emissions, combustion of petrochemicals, polymers, glues, paints, resins, synthetic fibers, furniture wax, and detergents (60).

Benzene is one of the 188 hazardous air pollutants listed by the EPA and has long been known to act as carcinogen of human blood components; In-fact the first case of "benzene associated lymphoma" was reported by French researchers in $1947(59,61)$. It has been well established that benzene decreases hematologic parameters, causes dysfunctional liver activity and enzymes and reduce circulating immunologic cell counts (62). Studies have also reported significant changes in developmental, reproductive, neurobehavioral, and respiratory function (62). Additionally, exposure to low levels of benzene can cause eye and skin irritation, drowsiness, dizziness, rapid heartbeat, headaches, nausea, tremors, confusion, and unconsciousness (63). Figures 1.11 and 1.12 provide a summary of existing information on the health effects of benzene (64). Benzene is classified by ATSDR and the U.S. EPA as a known human carcinogen (65). 


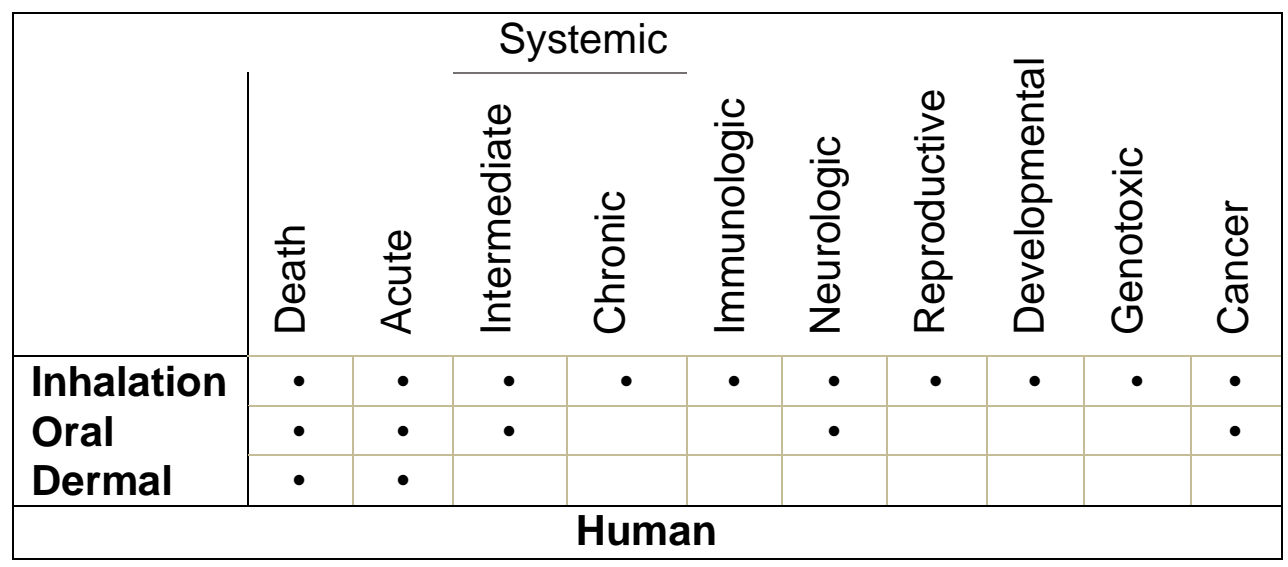

Figure 1.11. Existing Human Information on Health Effects of Benzene (64)

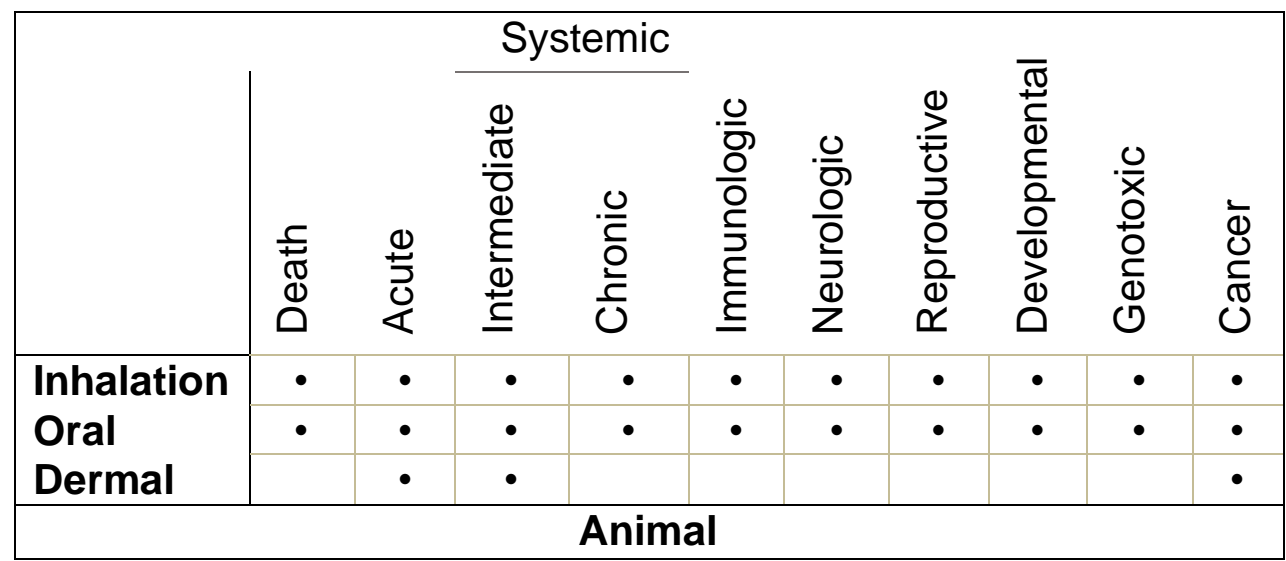

Figure 1.12. Existing Animal Information on Health Effects of Benzene (66)

\section{1-Bromopropane}

1-Bromopropane is a colorless liquid, which is slightly soluble in water (67). 1-Bromopropane is used in the production of commercial chemicals such as pesticides, flavors and fragrances, pharmaceuticals, and is used as a solvent in the adhesives, dry cleaning, vapor degreasing, and electronic and metal cleaning industries (68).

1-Bromopropane exposures can occur through dermal contact or through inhalation. Measurable quantities of 1-bromopropane have been detected in 
ambient air but most documented exposures are occupational related (68). Workers in industries that make or use 1-bromopropane, such as degreasing operations for cleaning metals, plastics, and electronic components, dry cleaning, asphalt production, aircraft maintenance, and synthetic fiber manufacturing, have the highest exposures (68).

Health effects of 1-bromopropane exposure generally involve the central nervous system and peripheral nervous systems and include symptoms such as respiratory and skin irritation, headache, nausea, and lower extremity numbness, pain, and weakness, blurred vision, nervousness, dizziness, and unconsciousness (68).

Currently 1-bromopropane is not listed as a toxicant or carcinogen in the U.S. but has been classified by the European Chemicals Agency as a reproductive toxicant and a "substance of very high concern" (69). Figures 1.13 and 1.14 provide a summary of existing information on the health effects of 1-bromopropane (68).

\begin{tabular}{|c|c|c|c|c|c|c|c|c|c|c|}
\hline & \multirow[b]{2}{*}{ 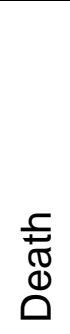 } & \multirow[b]{2}{*}{ 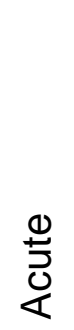 } & \multicolumn{3}{|c|}{ Systemic } & \multirow[b]{2}{*}{$\begin{array}{l}\text { 응 } \\
\text { 응 } \\
\frac{1}{3} \\
\text { 之) }\end{array}$} & \multirow[b]{2}{*}{$\begin{array}{l}\stackrel{0}{D} \\
\frac{0}{0} \\
\frac{0}{0} \\
\frac{0}{0} \\
\mathbb{0} \\
\simeq\end{array}$} & \multirow[b]{2}{*}{ 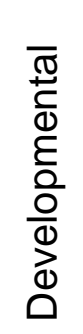 } & \multirow[b]{2}{*}{$\begin{array}{l}.0 \\
\frac{0}{x} \\
0 \\
0 \\
\frac{1}{0} \\
0\end{array}$} & \multirow[b]{2}{*}{$\begin{array}{l}\bar{\Phi} \\
\stackrel{\circlearrowright}{\mathbb{E}} \\
\text { U }\end{array}$} \\
\hline & & & 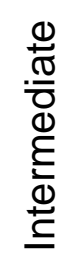 & $\begin{array}{l}\frac{0}{\bar{C}} \\
\frac{0}{\overline{0}} \\
0\end{array}$ & $\begin{array}{l}\frac{0}{\bar{O}} \\
\frac{0}{0} \\
\bar{\Xi} \\
\underline{E} \\
\underline{E}\end{array}$ & & & & & \\
\hline \multirow{3}{*}{$\begin{array}{l}\text { Inhalation } \\
\text { Oral } \\
\text { Dermal }\end{array}$} & & & - & & & • & • & & • & \\
\hline & & & & & & & & & & \\
\hline & & & & & & & & & & \\
\hline \multicolumn{11}{|c|}{ Human } \\
\hline
\end{tabular}

Figure 1.13. Existing Human Information on Health Effects of 1-Bromopropane (68) 


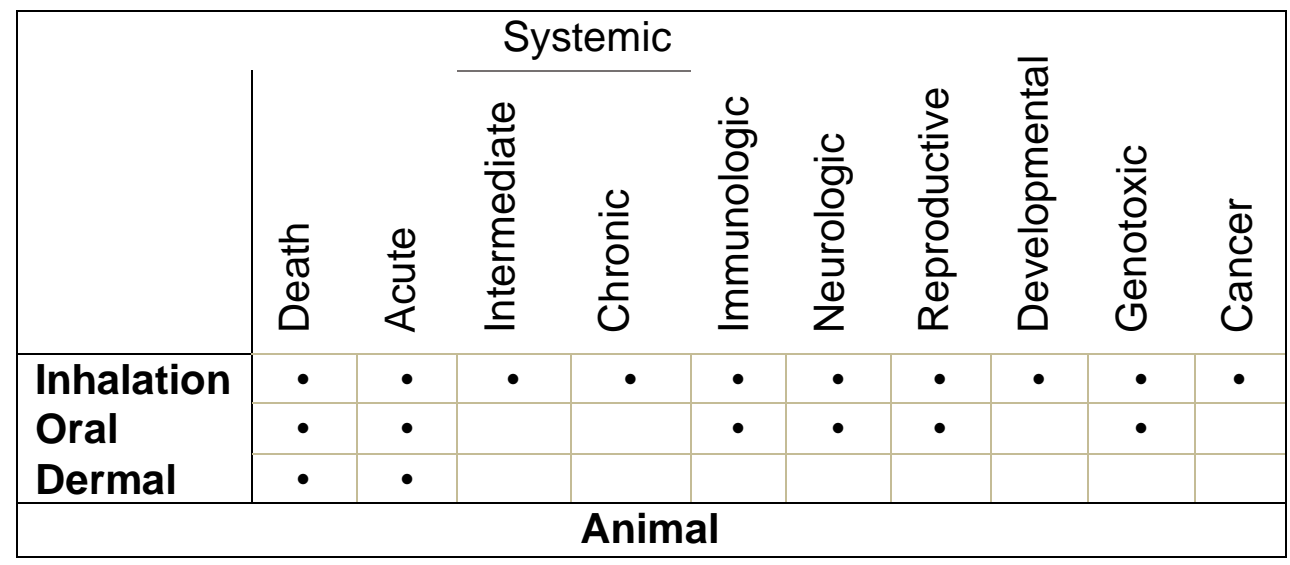

Figure 1.14. Existing Animal Information on Health Effects of 1-Bromopropane (70)

\section{1,3-Butadiene}

1,3-Butadiene, also commonly known as divinyl, is a synthetic, colorless, gas with a gasoline-like odor, which is insoluble in water but soluble in ethanol, ether, acetone, and benzene (71). 1,3-Butadiene is widely used in the manufacture of polymers and as an industrial chemical intermediate. Over half of the 1,3butadiene produced is used in the manufacture of man-made rubber (72).

1,3-Butadiene exposures predominantly occur through the inhalation route but can occur through ingestion and dermal contact (72). The most common exposure sources of 1,3-butadiene are; vehicle exhaust, gasoline fumes, tobacco smoke, burning wood, burning rubber and plastic, forest fires, and industrial releases (72). 1,3-Butadiene is a product of gasoline combustion and is therefore commonly found in ambient air samples particularly in the urban and suburban areas.

Acute 1,3-butadiene exposures generally cause mucosal and eye irritation, headache, fatigue, decreased blood pressure and pulse rate, central nervous 
system damage blurred vision, and unconsciousness. Chronic exposures have been associated with cardiovascular disease, leukemia, liver and blood disorders, other cancers, and central nervous system disturbances (72). 1,3-Butadiene is classified by ATSDR and the U.S. EPA as a known human carcinogen $(72,73)$. Figures 1.15 and 1.16 provide a summary of existing information on the health effects of 1,3-butadiene (72).

\begin{tabular}{|c|c|c|c|c|c|c|c|c|c|c|}
\hline & \multirow[b]{2}{*}{$\begin{array}{l}\text { F } \\
\text { D } \\
0\end{array}$} & \multirow[b]{2}{*}{$\begin{array}{l}\stackrel{0}{5} \\
\frac{0}{2}\end{array}$} & \multicolumn{2}{|c|}{ Systemic } & \multirow[b]{2}{*}{$\begin{array}{l}\frac{0}{O} \\
\frac{\mathrm{O}}{O} \\
\frac{1}{\bar{E}} \\
\underline{\underline{E}}\end{array}$} & \multirow[b]{2}{*}{$\begin{array}{l}\frac{0}{5} \\
\text { 응 } \\
\frac{1}{2} \\
\frac{1}{2}\end{array}$} & \multirow[b]{2}{*}{$\begin{array}{l}\stackrel{0}{D} \\
\frac{0}{0} \\
\overline{0} \\
\frac{0}{0} \\
\square \\
\square\end{array}$} & \multirow[b]{2}{*}{ 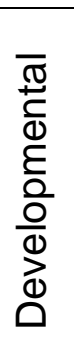 } & \multirow[b]{2}{*}{$\begin{array}{l}\frac{0}{x} \\
0 \\
0 \\
0 \\
0 \\
0\end{array}$} & \multirow[b]{2}{*}{$\begin{array}{l}\bar{\Phi} \\
\bar{C} \\
\bar{D} \\
\text { U }\end{array}$} \\
\hline & & & 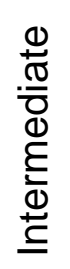 & $\begin{array}{l}\frac{0}{\frac{0}{D}} \\
\frac{0}{\frac{0}{L}} \\
\text { U }\end{array}$ & & & & & & \\
\hline \multirow{3}{*}{$\begin{array}{l}\text { Inhalation } \\
\text { Oral } \\
\text { Dermal }\end{array}$} & & - & & - & & & & & - & \\
\hline & & & & & & & & & & \\
\hline & \multicolumn{9}{|c|}{ Human } & \\
\hline
\end{tabular}

Figure 1.15. Existing Human Information on Health Effects of 1,3-Butadiene (72)

\begin{tabular}{|c|c|c|c|c|c|c|c|c|c|c|}
\hline & \multirow[b]{2}{*}{$\begin{array}{l}\text { 芜 } \\
\stackrel{D}{0}\end{array}$} & \multirow[b]{2}{*}{$\begin{array}{l}\frac{0}{5} \\
\frac{0}{2}\end{array}$} & \multicolumn{2}{|c|}{ Systemic } & \multirow[b]{2}{*}{ 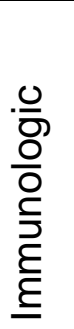 } & \multirow[b]{2}{*}{$\begin{array}{l}\frac{0}{0} \\
\frac{0}{0} \\
\frac{0}{2} \\
\frac{0}{2}\end{array}$} & \multirow[b]{2}{*}{ 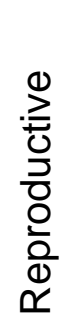 } & \multirow[b]{2}{*}{ 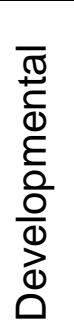 } & \multirow[b]{2}{*}{$\begin{array}{l}\frac{0}{x} \\
0 \\
\stackrel{0}{0} \\
\frac{1}{0} \\
0\end{array}$} & \multirow[b]{2}{*}{ 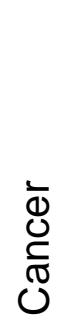 } \\
\hline & & & 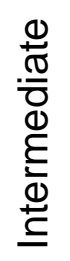 & $\begin{array}{l}\frac{0}{\frac{0}{\sigma}} \\
\frac{0}{\frac{0}{U}} \\
0\end{array}$ & & & & & & \\
\hline \multirow{3}{*}{$\begin{array}{l}\text { Inhalation } \\
\text { Oral } \\
\text { Dermal }\end{array}$} & - & - & - & - & - & - & - & - & - & - \\
\hline & & & & & & & & & & \\
\hline & & & & Anin & & & & & & \\
\hline
\end{tabular}

Figure 1.16. Existing Animal Information on Health Effects of 1,3-Butadiene (74)

\section{Carbon Disulfide}

Carbon disulfide is a colorless, highly flammable, poisonous liquid with a pleasant sweet-smelling odor. Carbon disulfide evaporates readily at room 
temperature; the vapor is more than two times heavier than air so it is often found a ground level. Carbon disulfide is used industrially as a non-polar solvent and organic building block in chemical reactions (75). Production of viscose rayon and cellophane film are the primary industrial uses of carbon disulfide. Carbon disulfide is also commonly used to dissolve rubber to produce tires and in the production of some pesticides (76).

The vast majority of carbon disulfide in the environment is from industrial releases. Once carbon disulfide makes it into the environment it rapidly evaporates and descends to ground level where it generally remains for a period of days to weeks until disturbed or degraded. Carbon disulfide does not bind tightly to soil, thus leaving it very mobile to move down into ground water (76). Generally, exposures to carbon disulfide occur in the workplace. Individuals' may be exposed by breathing, eating, drinking or by skin contact with contaminated soil, air, water or other substance that may contain it (76).

Exposure to carbon disulfide can result in symptoms such as dizziness, headaches, poor sleeping, fatigue, nausea, vomiting, nervousness, anorexia, lowweight, mood changes, psychosis, polyneuropathy, ocular changes, coronary heart disease, gastritis, kidney damage, liver damage, eye damage, skin burns, dermatitis, decreased sperm count and libido in men, and menstrual disturbances in women (75). Carbon disulfide is a central nervous system (CNS) disrupter and high dose exposures may lead to death due to CNS complications. Chronic exposures have been shown to cause behavioral and neurophysiological changes and have been epidemiologically linked to increased incidence of coronary heart 
disease and angina (75). Carbon disulfide has not been classified as a human carcinogen (75).

Carbon disulfide is lipophilic and is therefore more highly concentrated in organs such as the live and brain, where it is metabolized to thiocarbamates (76). Conjugation of carbon disulfide or carbonyl sulfide with endogenous glutathione results in the formation of thiozolidine-2-thione-4-carboxylic acid and 2oxythiazolidine-4-carboxylic acid, which are excreted in the urine (76). These thiocarbamates are potent antithyroid substances and may be involved in direct injury to the endothelium coupled with hypothyroidism (77). Within a few hours of inhalation exposure to carbon disulfide approximately $70 \%$ is metabolized excreted; the remaining $30 \%$ is slowly excrete or metabolized and then excreted in the urine (78). Figures 1.17 and 1.18 provide a summary of existing information on the health effects of carbon disulfide (76).

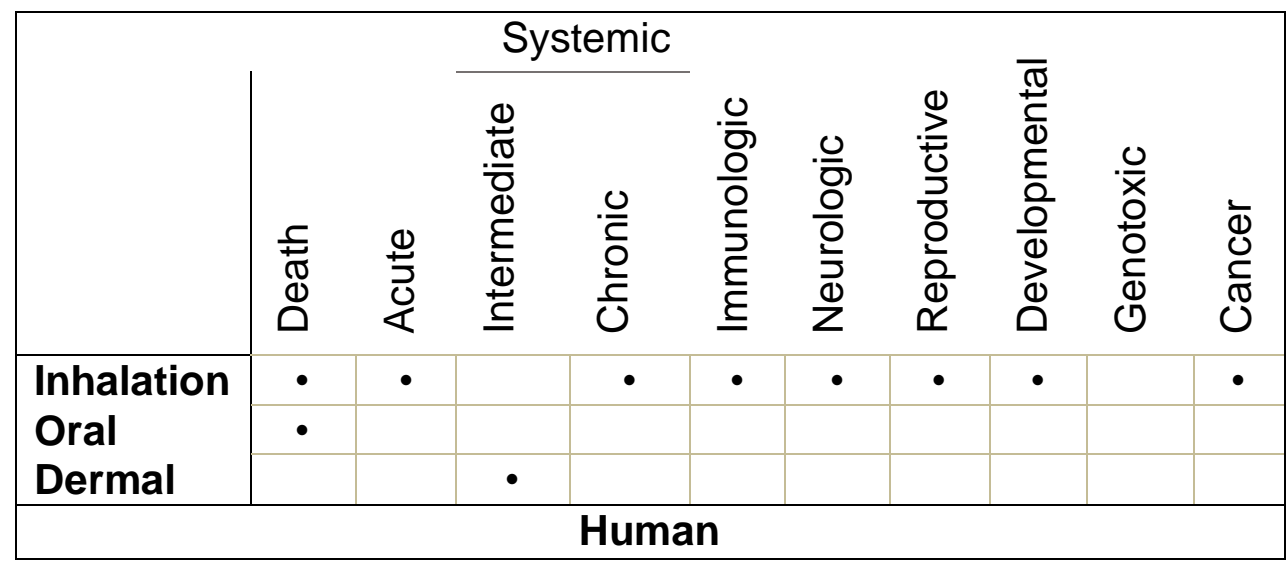

Figure 1.17. Existing Human Information on Health Effects of Carbon Disulfide (76) 


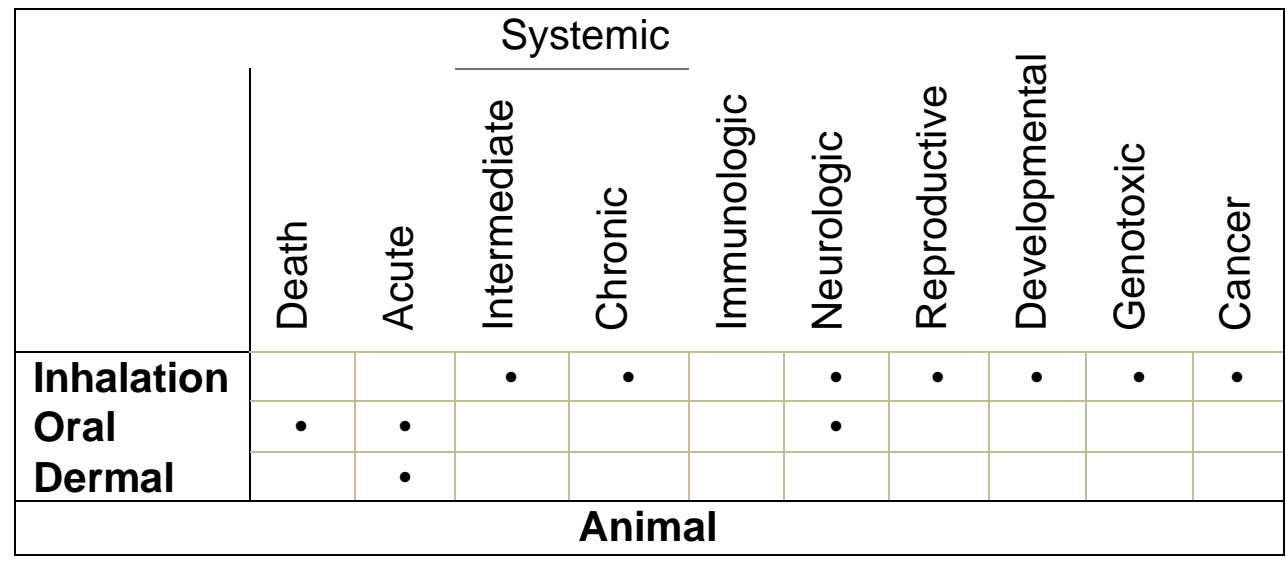

Figure 1.18. Existing Animal Information on Health Effects of Carbon Disulfide (79)

\section{Crotonaldehyde}

Crotonaldehyde is highly flammable, clear, colorless to straw colored liquid with a pungent suffocating odor. Crotonaldehyde is used in the manufacture of sorbic acid, a yeast and mold inhibitor and as a versatile intermediate in organic solvents and foodstuffs. Crotonaldehyde is used industrially as a chemical intermediate in the surfactants, textiles, paper, fuels, insecticides, and leather tanning industries. Crotonaldehyde is also used in chemical warfare (80).

Crotonaldehyde is emitted from the combustion of gasoline, the burning of wood and the burning of tobacco. The general public is most often exposed to crotonaldehyde by inhaling tobacco smoke, gasoline and diesel engine exhausts, and smoke from wood burning (80). Occupational inhalation and dermal exposures occur in industrial processes which utilize liquid crotonaldehyde in chemical reactions (80).

Symptoms of vapor inhalation, ingestion and dermal or eye absorption exposure include eye irritation, respiratory irritation, cough, burning sensation, 
sore throat, labored breathing, pulmonary edema, eye redness, pain, lacrimation, severe deep burns, diarrhea, nausea, vomiting. High-dose exposures of crotonaldehyde may cause a build-up of fluid in the lungs resulting in death (81). The possible carcinogenicity of crotonaldehyde is supported by studies of chronic exposure activities in F344 rats. These studies found that crotonaldehyde, $0.6 \mathrm{mM}$ dose, for 113 and 84 weeks, induced neoplastic lesions of the liver in 9 of 27 rats; 2 rats had hepatocellular carcinomas, and 9 rats had neoplastic nodules. It also caused altered liver cell foci in 23 of 27 rats (82).

The International Agency For Research On Caner evaluates crotonaldehyde as currently not classifiable as to the carcinogenicity to humans based on inadequate evidence in both human and animal studies but the American

Conference of Governmental Hygienists recognizes crotonaldehyde as a confirmed animal carcinogen and the U.S. Environmental Protection Agency classifies crotonaldehyde as a possible human carcinogen $(50,83,84)$.

\section{Cyanide}

Any chemical compound that contains a monovalently bound carbon and nitrogen atom is considered a cyanide. Cyanides are usually found joined with other chemicals to form compounds and can be found in various states of matter depending on their chemical composition. Hydrogen cyanide is a colorless gas with a faint, bitter, almond-like odor. Sodium cyanide and potassium cyanide are both white solids with a bitter, almond-like odor in damp air (85). Industrially cyanides are used in electroplating, metallurgy, organic chemicals production, 
photographic developing, manufacture of plastics, fumigation of ships, and some mining processes (85).

Cyanide can be found naturally in the soil and in a number of plants and foods such as: almonds, millet sprouts, lima beans, soy, spinach, bamboo shoots, and cassava roots. Exposure to cyanide in the general public commonly occurs from breathing air, drinking water, touching soil, or eating foods that contain or have been contaminated with cyanide. Additionally, smoking cigarettes and exposure to smoke from fires and fire places are a major sources of cyanide exposure (85). Exposures of airborne cyanide as low as $200 \mathrm{ppm}$ are sufficient to cause death in minutes (85).

Cyanide exposure causes the inhibition of the enzyme cytochrome c oxidase in the fourth complex of the electron transport chain, which prevents the transport of electrons from cytochrome $\mathrm{c}$ to oxygen resulting in the inability to aerobically produce adenosine triphosphate (ATP) for energy. The inability to aerobically produce ATP results in histotoxic hypoxia, which is more pronounced in tissues that rely heavily on aerobic respiration, notably the heart and central nervous system. Symptoms of cyanide exposure are; rapid difficulty breathing, seizures, unconsciousness, chest pain, vomiting, blood changes, headaches, and enlargement of the thyroid gland. Thiocyanate, the main metabolite of cyanide, is a reactive metabolite and is known to be responsible for damage to the thyroid gland and suspected to be the cause of the endocrine effects associated with cyanide exposure (85). The carcinogenic effects of cyanide are not well studied. Animal studies have demonstrated metabolic effects, decreased serum albumin 
and lowered calcium and potassium levels, reductions in mitochondrial respiratory control ratios, and reduced tissue ATP levels in the liver and heart $(86,87)$. Figures 1.19 and 1.20 provide a summary of existing information on the health effects of cyanide (85).

\begin{tabular}{|c|c|c|c|c|c|c|c|c|c|c|}
\hline & \multirow[b]{2}{*}{ 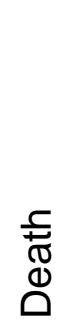 } & \multirow[b]{2}{*}{ 壱 } & \multicolumn{2}{|c|}{ Systemic } & \multirow[b]{2}{*}{ 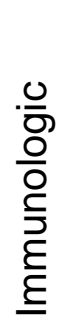 } & \multirow[b]{2}{*}{$\begin{array}{l}\frac{0}{2} \\
\frac{0}{0} \\
\frac{0}{3} \\
\stackrel{0}{Z}\end{array}$} & \multirow[b]{2}{*}{$\begin{array}{l}0 \\
\stackrel{0}{0} \\
\frac{0}{0} \\
\frac{0}{0} \\
\frac{0}{0} \\
\simeq\end{array}$} & \multirow[b]{2}{*}{ 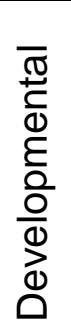 } & \multirow[b]{2}{*}{$\begin{array}{l}\frac{0}{x} \\
0 \\
0 \\
0 \\
0 \\
0 \\
0\end{array}$} & \multirow[b]{2}{*}{$\begin{array}{l}\bar{\Phi} \\
\overline{0} \\
\bar{\varpi} \\
0\end{array}$} \\
\hline & & & 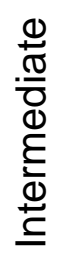 & $\begin{array}{l}\frac{0}{\frac{0}{D}} \\
\frac{0}{\frac{0}{U}}\end{array}$ & & & & & & \\
\hline \multirow{3}{*}{$\begin{array}{l}\text { Inhalation } \\
\text { Oral } \\
\text { Dermal }\end{array}$} & • & - & & - & & - & & & & \\
\hline & - & - & & - & & - & & & & \\
\hline & - & - & & & & - & & & & \\
\hline
\end{tabular}

Figure 1.19. Existing Human Information on Health Effects of Cyanide (85)

\begin{tabular}{|c|c|c|c|c|c|c|c|c|c|c|}
\hline & \multirow[b]{2}{*}{$\begin{array}{l}\text { F } \\
\text { స్ } \\
\stackrel{1}{0}\end{array}$} & \multirow[b]{2}{*}{ 壳 } & \multicolumn{2}{|c|}{ Systemic } & \multirow[b]{2}{*}{ 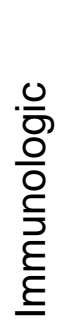 } & \multirow[b]{2}{*}{$\begin{array}{l}\frac{0}{3} \\
\frac{0}{0} \\
\frac{0}{2} \\
\frac{0}{2}\end{array}$} & \multirow[b]{2}{*}{ 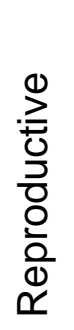 } & \multirow[b]{2}{*}{ 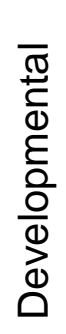 } & \multirow[b]{2}{*}{$\begin{array}{l}\frac{0}{x} \\
\frac{0}{0} \\
\frac{0}{0} \\
0\end{array}$} & \multirow[b]{2}{*}{$\begin{array}{l}\bar{\Phi} \\
\bar{U} \\
\bar{C} \\
0\end{array}$} \\
\hline & & & 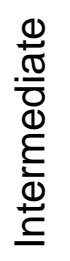 & 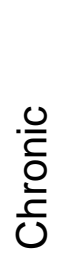 & & & & & & \\
\hline \multirow{3}{*}{$\begin{array}{l}\text { Inhalation } \\
\text { Oral } \\
\text { Dermal }\end{array}$} & - & - & - & & & - & & & & \\
\hline & - & - & - & - & - & - & - & - & - & \\
\hline & - & - & & & & - & & & & \\
\hline
\end{tabular}

Figure 1.20. Existing Animal Information on Health Effects of Cyanide (88)

\section{$N, N$-Dimethylformamide}

$\mathrm{N}, \mathrm{N}$-Dimethylformamide, also known as dimethylformamide or DMF for short, is a clear liquid, which is miscible with water and the majority of organic liquids. N, N-Dimethylformamide is widely used industrially as a solvent, additive, 
and/or as an intermediate in chemical processing due to its miscibility with most common organic solvents (89). N, N-Dimethylformamide is often used in the production of fibers, films, and surface coatings. The primary end users of $\mathrm{N}, \mathrm{N}$ dimethylformamide are pharmaceutical, electronic components, butadiene, and urethanes manufactures. It is also used as a resin cleanup solvent, reaction solvent, and processing solvent in the manufacture of polyimides, optical brighteners, semipermeable membranes, and pesticides (90).

$\mathrm{N}, \mathrm{N}$-Dimethylformamides' wide application has led to its selection as one of the four priority compounds for human field studies by the National Toxicology Program (NTP) of the US National Institute of Environmental Health Sciences (NIEHS). Exposures to N, N-dimethylformamide occurs most commonly in occupational setting through inhalation, or dermal contact. The general public has the greatest potential for exposure to $\mathrm{N}, \mathrm{N}$-dimethylformamide though pesticide use and exposure to contaminated air in the vicinity of industrial point sources (91).

Chronic low dose exposures to $\mathrm{N}, \mathrm{N}$-dimethylformamide generally result in symptoms such as gastrointestinal distress, abdominal pain, nausea, vomiting, fatigue, headache, dizziness, skin irritation, conjunctivitis, changes in blood pressure, liver injury, tachycardia \& electrocardiography (ECG) abnormalities. Very low dose exposure to N, N-dimethylformamide, may cause alcohol intolerance.

There is limited evidence that $\mathrm{N}, \mathrm{N}$-dimethylformamide is carcinogenic for humans and the International Agency for Research on Cancer has evaluated N, $\mathrm{N}$-dimethylformamide as not classifiable as a carcinogen in humans (Group 3) 
(50). Studies have reported increased incidence of testicular tumors, and tumors of the buccal cavity and pharynx, as well as increased frequency of miscarriages with chronic N, N-dimethylformamide (92). Additionally, excess mortality from ischemic heart disease and cardiac disturbances, including tachycardia and palpitations, have been reported in studies of $\mathrm{N}, \mathrm{N}$-dimethylformamide-exposed workers. Genotoxic effects have also been reported in humans, while reproductive, developmental, and neurological effects have been observed in animal studies (91).

The metabolism of $\mathrm{N}, \mathrm{N}$-dimethylformamide takes place primarily in the liver, where it is metabolized by the microsomal cytochrome $\mathrm{p}-450$ into mainly $\mathrm{N}$ hydroxymethyl- $\mathrm{N}$-methylformamide (HMMF), which is unstable and readily decomposes to $\mathrm{N}$-methyformamide (NMF). However, the detailed mechanism of its exact toxicity remain unclear (92).

\section{Ethylbenzene}

Ethylbenzene is a colorless, highly flammable liquid, with an odor similar to gasoline. Ethylbenzene is more dense that air but less dense than water and insoluble in water; hence ethylbenzene floats on the surface of water (93).

Ethylbenzene is commonly used industrially as a solvent in the production of other chemicals. One of the main consumers of ethylbenzene is the manufacture of styrene where it is used as an intermediate and in the production of polystyrene. Ethylbenzene is also used in the production of inks, insecticides, jet fuel, and paints, and can be found naturally in products such as coal tar and petroleum (63). 
Ethylbenzene is released into the environment from burning oil, gas, and coal, and from discharges of ethylbenzene from factories. Ethylbenzene is most commonly found in the air as a vapor and the general public is exposed to ethylbenzene from gasoline or from the use of pesticides, carpet glues, varnishes, pesticides, inks, and paints, and use of tobacco, which all contain ethylbenzene (63). Heavily trafficked areas and industrial manufacturing are known to be large sources of ambient ethylbenzene exposure (63).

High dose exposures of ethylbenzene are known to cause eye and throat irritation, dizziness, and distressed breathing. Ethylbenzene is known to affect the central nervous system and cause liver damage. In vivo studies have demonstrated deleterious effects of ethylbenzene on the inner ear, kidney function, liver, and kidney tumor development (94). Ethylbenzene is classified as a possible carcinogen by the International Agency for Research on Cancer (IRAC), and as a confirmed animal carcinogen with unknown relevance to humans by the American Conference of Governmental Industrial Hygienists (ACGIH), however the Environmental Protection Agency (EPA) has not determined ethylbenzene to be a carcinogen $(93,94)$. In humans ethylbenzene is mainly metabolized in the liver to mandelic acid (65-70\%) and phenylglyoxylic acid (20-25\%) (95). Figures 1.21 and 1.22 provide a summary of existing information on the health effects of ethylbenzene (94). 


\begin{tabular}{|c|c|c|c|c|c|c|c|c|c|c|}
\hline & \multirow[b]{2}{*}{ 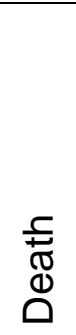 } & \multirow[b]{2}{*}{ 莺 } & \multicolumn{2}{|c|}{ Systemic } & \multirow[b]{2}{*}{ 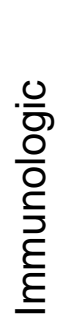 } & \multirow[b]{2}{*}{$\begin{array}{l}\frac{0}{2} \\
\frac{0}{0} \\
\frac{0}{3} \\
\stackrel{0}{Z}\end{array}$} & \multirow[b]{2}{*}{ 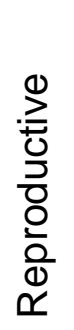 } & \multirow[b]{2}{*}{ 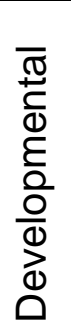 } & \multirow[b]{2}{*}{$\begin{array}{l}. \frac{0}{x} \\
0 \\
0 \\
\frac{0}{0} \\
0 \\
0\end{array}$} & \multirow[b]{2}{*}{$\begin{array}{l}\bar{\Phi} \\
\bar{U} \\
\overline{\mathbb{D}} \\
\mathcal{U}\end{array}$} \\
\hline & & & 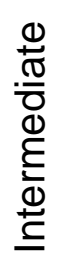 & $\begin{array}{l}\frac{0}{\frac{U}{\sigma}} \\
\frac{0}{\frac{0}{U}}\end{array}$ & & & & & & \\
\hline Inhalation & & - & & - & & - & & & & - \\
\hline $\begin{array}{l}\text { Oral } \\
\text { Dermal }\end{array}$ & & & & & & & & & & \\
\hline
\end{tabular}

Figure 1.21. Existing Human Information on Health Effects of Ethylbenzene (94)

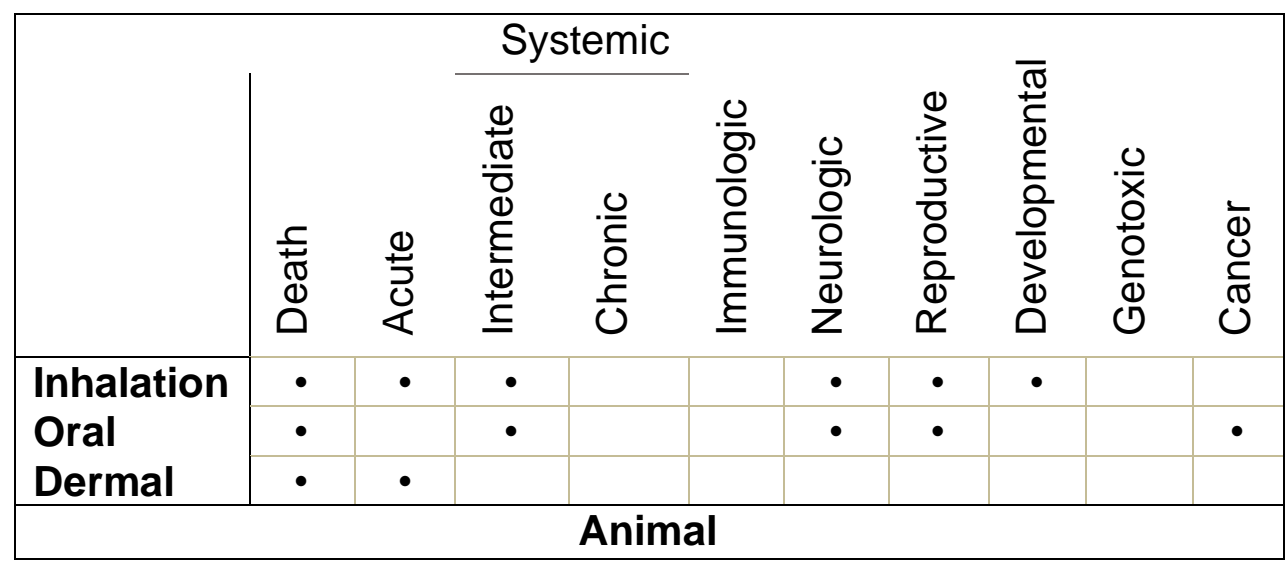

Figure 1.22. Existing Animal Information on Health Effects of Ethylbenzene (96)

\section{Styrene}

Styrene, also known as vinyl benzene, is a colorless oily liquid with a strong sweet aromatic smell. Styrene is primarily a man-made chemical used in the production of rubber and plastics and is present in organic combustion products such as cigarette smoke and automobile exhaust. Common consumer products that contain styrene include: packaging materials, insulation products, fiberglass, plastics, many "food use" items and carpeting materials. While styrene is primarily a man-made chemical, it can also be found naturally existing in a wide variety of 
foods such as fruits, vegetables, nuts, beverages, and meats and is used as a flavoring ingredient and an ion-exchange resins in food processing $(63,97)$.

Styrene can be found in air, soil, and water, but is not commonly found in drinking water. People are most often expose to styrene though contaminated air, food, or water but can also be absorbed through the skin. Point source releases to ambient air occur in the vicinity of styrene manufactures, while other common exposure sources include automobile exhaust, cigarette smoke, and photocopy machines (98). Occupational exposures are not uncommon; people working in the reinforced-plastics industry have the highest potential for exposure. Additionally, individuals who work in styrene polymerization, rubber manufacturing, and styrene-polyester resin facilities and workers at photocopy centers may also be exposed to styrene (98).

Acute high dose exposures, generally occurring in occupational settings, commonly involve the nervous system and include symptoms such as: changes in color vision, irritation of the eyes, nose, and throat, lethargy, feeling drunk and/or dizzy, headache, slowed reaction time, concentration problems, and balance problems. Chronic exposures have also reported nervous system affects but with more sever neurological complications such as: depression, concentration problems, muscle weakness, personality changes, and anxiety. In-vivo studies have shown significant liver damage, reproductive and developmental problems. The Agency for Toxic Substances and Disease Registry has classified styrene as reasonably anticipated to be a human carcinogen and the International Agency for Research on Cancer has classified styrene as possibly carcinogenic to humans 
(Group 2B) $(48,50,98)$. Metabolic activation is considered to be a critical step for styrene-induced toxicity. The CYP2E1 has been identified as the main cytochrome P450 isoform involved in human styrene metabolism. Styrene-7,8-oxide is a primary oxidative metabolite and 4-vinylphenol, a phenolic metabolite formed by aromatic hydroxylation has also been detected as a styrene metabolite $(99,100)$. Figures 1.23 and 1.24 provide a summary of existing information on the health effects of styrene (98).

\begin{tabular}{|c|c|c|c|c|c|c|c|c|c|c|}
\hline & \multirow[b]{2}{*}{$\begin{array}{l}\text { F } \\
\mathbb{\varpi} \\
\mathbb{D} \\
0\end{array}$} & \multirow[b]{2}{*}{$\begin{array}{l}\frac{0}{5} \\
\frac{0}{2}\end{array}$} & \multicolumn{2}{|c|}{ Systemic } & \multirow[b]{2}{*}{$\begin{array}{l}\text { 응 } \\
\text { 응 } \\
\underline{\bar{E}} \\
\underline{E}\end{array}$} & \multirow[b]{2}{*}{$\begin{array}{l}\frac{0}{3} \\
\text { 응 } \\
\frac{0}{3} \\
\text { Z }\end{array}$} & \multirow[b]{2}{*}{ 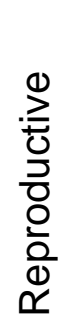 } & \multirow[b]{2}{*}{ 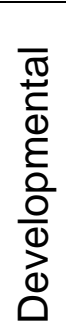 } & \multirow[b]{2}{*}{$\begin{array}{l}\frac{0}{x} \\
0 \\
0 \\
0 \\
0 \\
0 \\
0\end{array}$} & \multirow[b]{2}{*}{$\begin{array}{l}\bar{\Phi} \\
\bar{D} \\
\mathbb{N} \\
\mathcal{J}\end{array}$} \\
\hline & & & 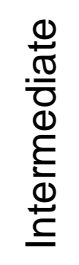 & $\begin{array}{l}\frac{.}{\bar{C}} \\
\stackrel{0}{\frac{D}{U}} \\
\end{array}$ & & & & & & \\
\hline Inhalation & & • & - & - & - & - & - & - & - & - \\
\hline $\begin{array}{l}\text { Oral } \\
\text { Dermal }\end{array}$ & & & & & & & & & & \\
\hline & & & & Hum & & & & & & \\
\hline
\end{tabular}

Figure 1.23. Existing Human Information on Health Effects of Styrene (101)

\begin{tabular}{|c|c|c|c|c|c|c|c|c|c|c|}
\hline & \multirow[b]{2}{*}{ 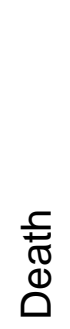 } & \multirow[b]{2}{*}{$\frac{0}{5}$} & \multicolumn{2}{|c|}{ Systemic } & \multirow[b]{2}{*}{ 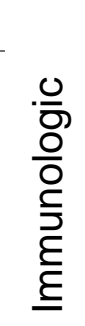 } & \multirow[b]{2}{*}{$\begin{array}{l}\frac{0}{2} \\
\frac{0}{0} \\
\frac{0}{3} \\
\frac{0}{2}\end{array}$} & \multirow[b]{2}{*}{ 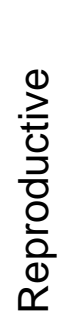 } & \multirow[b]{2}{*}{ 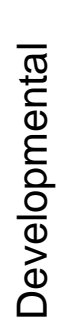 } & \multirow[b]{2}{*}{$\begin{array}{l}.0 \\
\frac{0}{X} \\
0 \\
0 \\
0 \\
0 \\
0 \\
0\end{array}$} & \multirow[b]{2}{*}{$\begin{array}{l}\bar{\Phi} \\
\frac{0}{\mathbb{N}} \\
\mathcal{O}\end{array}$} \\
\hline & & & 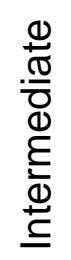 & $\begin{array}{l}\frac{0}{\frac{0}{D}} \\
\frac{0}{\bar{O}} \\
0\end{array}$ & & & & & & \\
\hline \multirow{3}{*}{$\begin{array}{l}\text { Inhalation } \\
\text { Oral } \\
\text { Dermal }\end{array}$} & - & - & - & - & & - & • & - & - & - \\
\hline & - & - & - & - & - & - & - & - & - & - \\
\hline & & - & & & & & & & & \\
\hline \multicolumn{11}{|c|}{ Animal } \\
\hline
\end{tabular}

Figure 1.24. Existing Animal Information on Health Effects of Styrene (98)

\section{Propylene Oxide}

Propylene oxide, also known as epoxypropae, is a clear colorless volatile 
liquid with a sweet, alcoholic, and ether-like odor. Propylene oxide vapor is heavier than air and highly soluble in water (102). Propylene oxide is produced on a large scale industrially with the majority of production being converted to polyether polyols, building blocks in the production of polyurethane plastics (102). Propylene oxide is also used in the production of propylene glycol and as a fumigant of foodstuffs and plastic medical instruments and as a herbicide, as a solvent, and in the preparation of lubricants, surfactants, and oil demulsifiers $(103,104)$.

Occupational exposures of propylene oxide through both inhalation and dermal routes in industries that produce, store, transport or use propylene oxide may be significant. The general public may be exposed to propylene oxide by point source releases from industrial plant as well as from the use of herbicides, plastics, or the consumption of fumigated food products $(102,103)$.

Acute propylene oxide exposure causes eye, skin, and respiratory irritation and lesions, coughing, dyspnea (difficulty in breathing), and pulmonary edema. Dermal contact may result in delayed burns and lesions. Propylene oxide is a known mild central nervous system depressant and acute high dose exposures may result in headache, motor weakness, incoordination, ataxia, and coma in humans. Chronic exposures in animal studies have shown neurological and tumorcausing effects, decreased weight gain, and increased mortality. The U.S. Environmental Protection Agency and the International Agency for Research on Cancer have classified propylene oxide as a Group B2, probable human carcinogen and the American Conference of Governmental Industrial Hygienists have classified propylene oxide as a confirmed animal carcinogen with unknown 
relevance to humans $(49,50,103)$. Propylene oxide reacts with DNA at neutral PH to yield two principal products, n-7-(2-hydroxypropyl)guanine and n-3-(2hydroxypropyl)adenine (50).

\section{Tetrachloroethylene}

Tetrachloroethylene, also known as perchloroethylene, is a colorless sweet smelling liquid that is volatile, highly stable, and nonflammable (105). It is a manmade chemical that is widely used in dry-cleaning fabrics and metal degreasing operations (106). Tetrachloroethylene is an excellent solvent and is used as a chemical building block in the production of many consumer products such as: water repellents, silicone lubricants, fabric finishers, spot removers, adhesives, and wood cleaners (63).

People usually are exposed to tetrachloroethylene by breathing contaminated air. Tetrachloroethylene is released into the environment, air, soil, and water, from industrial sources and/or industrial waste sites, but can also be exposed by drinking contaminated water and eating contaminated food. Tetrachloroethylene can stay in the air for a long time before it is broken down and easily travels through the soil and into ground water supplies $(63,106,107)$.

Acute high dose exposures of tetrachloroethylene may result in: skin irritation, dizziness, headache, sleepiness, confusion, nausea, difficulty in speaking and walking, mood and behavioral changes, unconsciousness, liver and kidney damage, and even death (63). Chronic exposure to tetrachloroethylene can cause nervous system problems, such as memory loss and concentration problems, impairments in cognitive and motor neurobehavioral functioning, and 
color vision impairment. Tetrachloroethylene exposure may also cause adverse effects in the kidney, liver, immune system, and hematologic system, and on developmental and reproductive systems. Studies of people exposed in the workplace have found associations with several types of cancer including bladder cancer, non-Hodgkin lymphoma, multiple myeloma, as well as reproductive complications such as menstrual disorders, altered sperm structure, and reduced fertility and inconclusive evidence of birth defects (106). Studies of dry-cleaning workers exposed to tetrachloroethylene have shown associations between exposure to tetrachloroethylene and several types of cancer, specifically bladder cancer, non-Hodgkin lymphoma and multiple myeloma. There is also limited evidence suggestive of associations with esophageal, kidney, cervical and breast cancer (106). In vivo studies of tetrachloroethylene exposures have reported mononuclear cell leukemias and liver and kidney tumors in male rats (106). The Agency for Toxic Substances and Disease Registry classifies tetrachloroethylene as likely to be carcinogenic in humans by all routes of exposure and the International Agency for Research on Cancer classifies tetrachloroethylene as probably carcinogenic to humans (Group 2A) $(50,107)$.

The disposition of an absorbed dose of tetrachloroethylene occurs primarily through pulmonary excretion; metabolism is less important than for other chlorinated solvents, such as trichloroethylene. Mass-balance studies in rats with $14 \mathrm{C}$-labeled tetrachloroethylene indicated that $70 \%$ or more of an oral or inhaled dose can be recovered in expired air as the parent compound. The next most important excreted fraction occurs in urine and feces, which may collectively 
account for up to $23 \%$ of an administered dose. A small portion of the dose (less than $3 \%$ ) may be converted to $\mathrm{CO} 2$ and exhaled. Most of the radioactivity recovered in urine can be attributed to formation of trichloroacetic acid, a nonvolatile metabolite of tetrachloroethylene that is excreted primarily in urine. This general pattern of disposition of tetrachloroethylene appears to be consistent after both oral and inhalation dosing (108). Figures 1.25 and 1.26 provide a summary of existing information on the health effects of tetrachloroethylene (107).

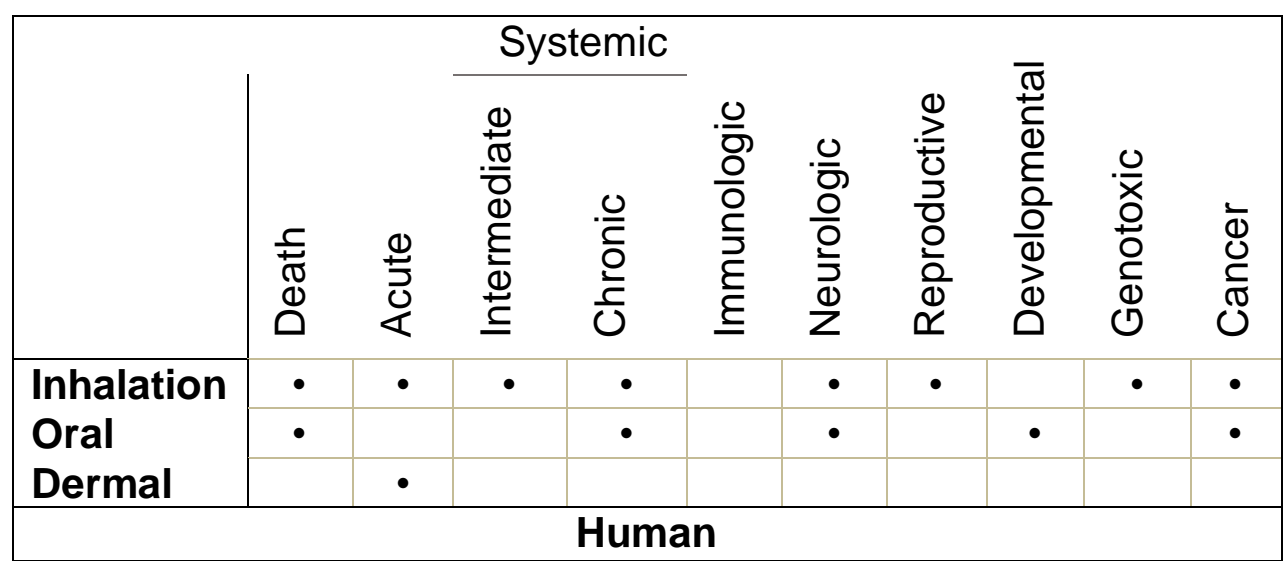

Figure 1.25. Existing Human Information on Health Effects of Tetrachloroethylene (107)

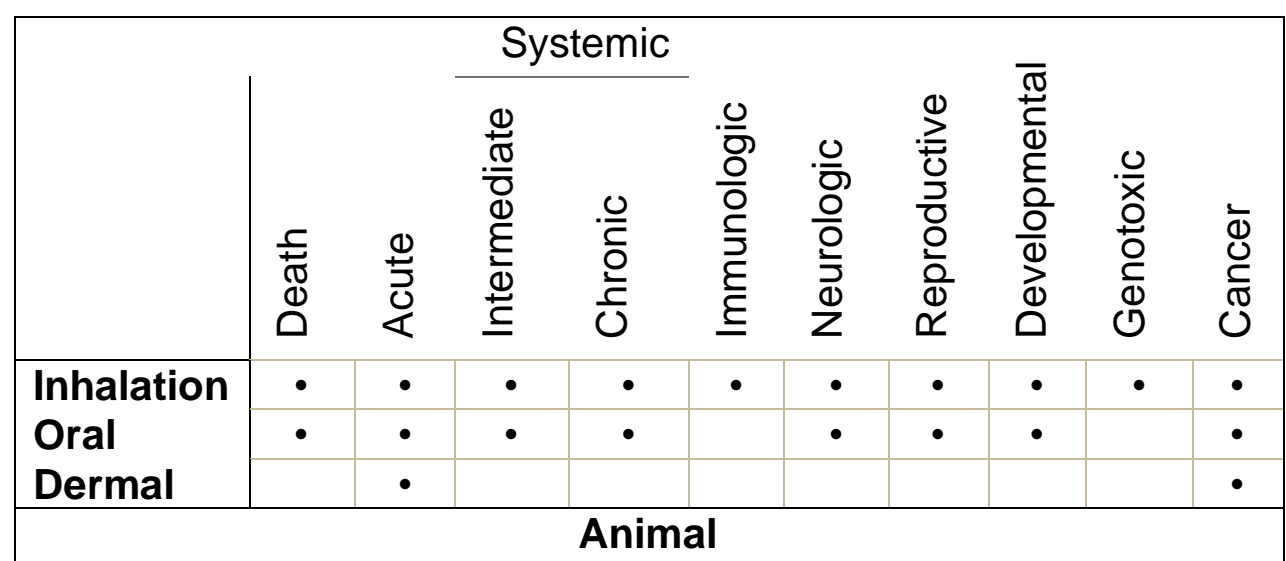

Figure 1.26. Existing Animal Information on Health Effects of Tetrachloroethylene (109) 


\section{Toluene}

Toluene is a colorless, aromatic liquid with a distinct odor (110). It is less dense than, and insoluble in water. Toluene occurs naturally in petroleum and coal tar and is synthesized industrially for use as a solvent (111).

Toluene is an important industrial solvent and is widely used in making paints, paint thinners, fingernail polish, lacquers, adhesives, and rubber, and in some printing and leather tanning processes. Additionally, toluene is a major component in jet fuel (63). Toluene's extensive use in industry and transportation make it quite abundant in the environment and therefore industrial exposure sources are virtually unavoidable. In addition to the abundant industrial emission exposures, the general public is exposed to toluene via numerous common-use products. Toluene is present in every-day products such as automobile exhaust, glues, solvents, and tobacco smoke (63).

Acute high dose exposures of toluene in humans have been reported to cause light-headiness, dizziness, sleepiness, unconsciousness, kidney damage and death (63). Animal studies have shown that moderate or high levels of toluene can cause liver, kidney, and lung dysfunction and damage (63). Additionally, chronic low dose exposures have been reported to include effects on the central nervous system, depression, difficulty concentrating, muscle weakness, memory loss, and personality changes (111). Figures 1.27 and 1.28 provide a summary of existing information on the health effects of toluene (112). 


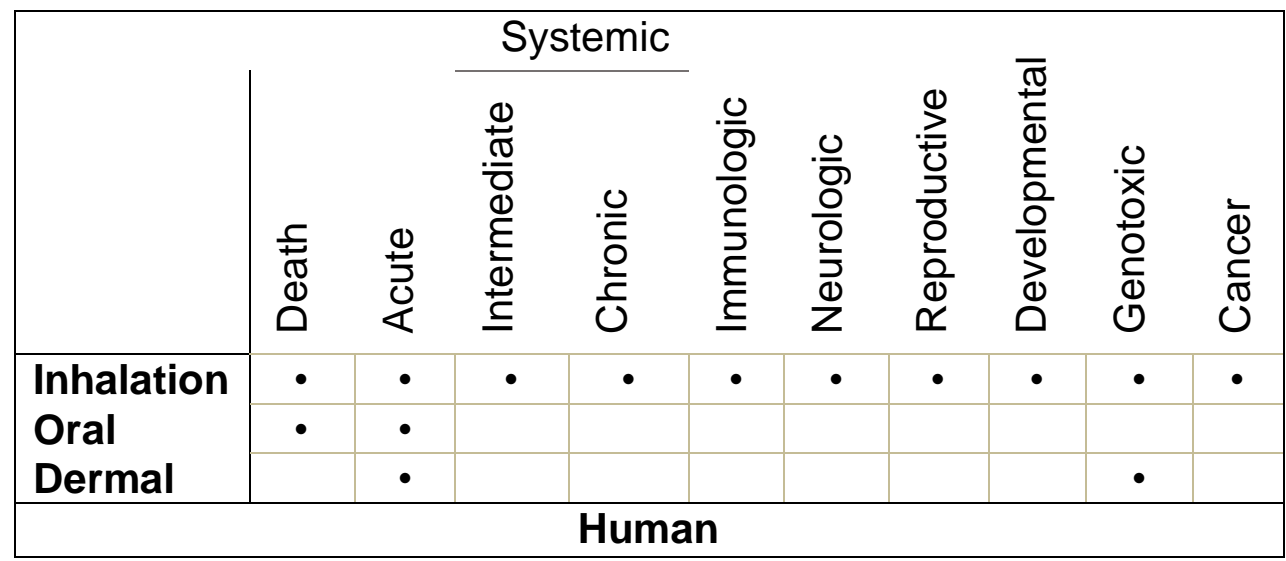

Figure 1.27. Existing Human Information on Health Effects of Toluene (112)

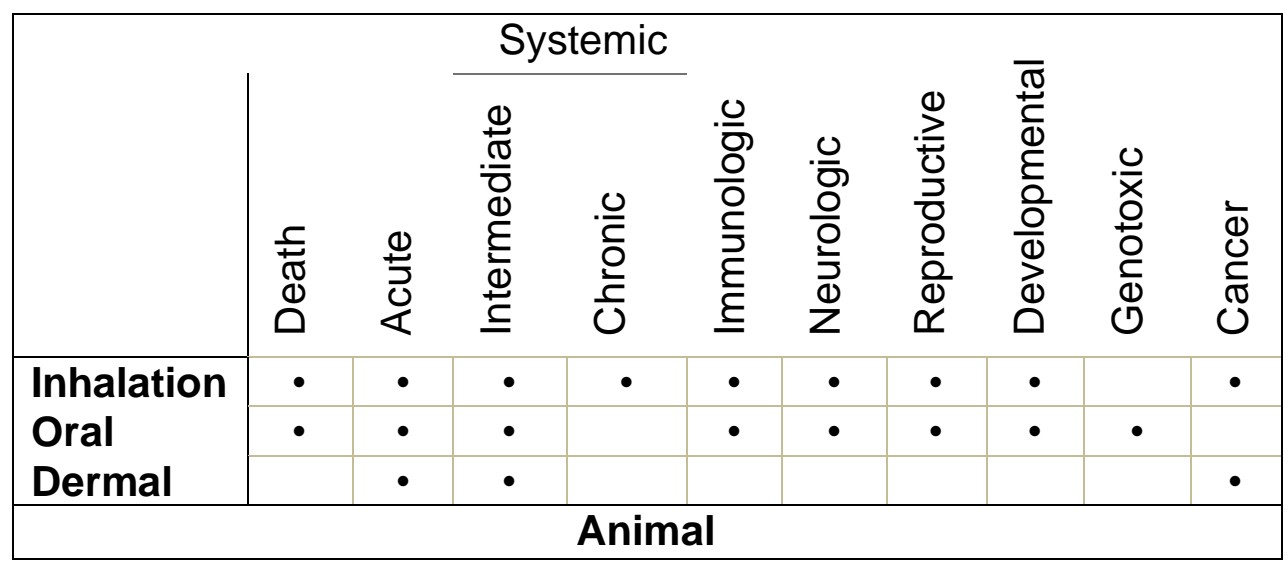

Figure 1.28. Existing Animal Information on Health Effects of Toluene (113)

\section{Trichloroethylene}

Trichloroethylene, also known as trichloroethene, is a man-made sweet smelling, nonflammable, colorless liquid. Trichloroethylene is commonly used as an industrial solvent and degreaser for metal parts, as a building-block in the production of the refrigerant HFC-134a, and is used as an inhalation anesthetic for short surgical procedures where light anesthesia with good analgesia is required. Trichloroethylene has also been used as an extraction solvent for greases, oils, fats, waxes, and tars; by the textile processing industry to scour cotton, wool, and other fabrics; in dry cleaning operations; and as a component of adhesives, 
lubricants, paints, varnishes, paint strippers, pesticides, and cold metal cleaners $(114,115)$.

People are exposed to trichloroethylene from breathing contaminated air or eating, drinking, or touching contaminated foodstuffs or consumer products. People who live near facilities that utilize or manufacture trichloroethylene or individuals who live near hazardous waste sites may have higher exposures to trichloroethylene. Individuals who live near these locations are at higher risk not only from breathing contaminated air but also because trichloroethylene breaks down slowly in the soil and in the water, making these sources additional potential chronic exposure sources (115). In fact ATSDR reports that trichloroethylene is the most frequently reported organic contaminant in groundwater (115). The general public is most likely to be exposed to trichloroethylene by drinking trichloroethylene-contaminated water. Individuals may also be exposed to trichloroethylene by breathing trichloroethylene released into the air from contaminated water. Individuals who work in the degreasing industry or other industry where trichloroethylene is used, may be exposed by breathing in trichloroethylene-contaminated air or by contacting the chemical with your skin.

Acute effects of trichloroethylene generally involve the central nervous system and include symptoms such as: sleepiness, fatigue, headache, confusion, hearing, sight and balance, changes and feelings of euphoria; and effects of the liver, kidneys, lung, heart, gastrointestinal system, and skin have been noted (116). Relatively short in vivo trichloroethylene exposures have resulted in harmful effects on the nervous system, liver, respiratory system, kidneys, blood, immune system, 
heart, and body weight. Chronic occupational exposure reports are similar to acute exposure reports and include symptoms of the central nervous system such as: dizziness, headache, sleepiness, nausea, confusion, blurred vision, facial numbness, and weakness. Chronic exposures have also been shown to affect the liver, kidneys, immune and endocrine systems and have been reported to cause scleroderma (a systemic autoimmune disease). Interestingly, studies have shown that simultaneous alcohol consumption and trichloroethylene inhalation increases the toxicity of trichloroethylene in humans. Epidemiologic studies have suggested trichloroethylene exposure may be associated with increased incidence of mismanages and congenital heart disease in children and animal studies have reported developmental effects from exposure to trichloroethylene and its metabolites (trichloroacetic acid [TCA] and dichloroacetic acid [DCA]) $(63,115$, 116).

Several well-designed epidemiologic studies have been published reporting trichloroethylene exposure to be associated with several types of cancers in humans, including; especially kidney, liver, cervix, and lymphatic system. These results are supported by molecular epidemiology studies showing specific renal cell mutations found primarily in renal cell carcinoma patients exposed to trichloroethylene, and in vivo studies showing increases in lung, liver, kidney, and testicular tumors and lymphoma (116). The Agency for Toxic Substances and Disease Registry and the International Agency for Research on Cancer (IARC) have determined that trichloroethylene is "carcinogenic to humans" $(50,115)$. 
Early studies showed that approximately $11 \%$ of absorbed trichloroethylene is eliminated unchanged from the lungs and another $2 \%$ is metabolized and eliminated by the lungs; $58 \%$ of absorbed trichloroethylene is eliminated in the urine and about $8 \%$ of the retained trichloroethylene is excreted as metabolites in the feces. Other more recent studies have shown up to $73 \%$ of the trichloroethylene retained after inhalation could be recovered in the urine. The trichloroethylene metabolites found in the urine are; monochloroacetic acid, 4\%; trichloroacetic acid, 19\%; \& trichloroethanol, $50 \%$. In humans, excretion of the metabolites of trichloroethylene is fastest for monochloroacetic acid, intermediate for trichloroethanol, and the slowest for trichloroacetic acid. Trichloroethylene is metabolized by hepatic P450 system and to a much lesser degree by direct conjugation with glutathione. The metabolite trichloroacetic acid is considered a principal metabolite responsible for trichloroethylene-induced liver cancer in mice (117). Figures 1.29 and 1.30 provide a summary of existing information on the health effects of trichloroethylene (115).

\begin{tabular}{|c|c|c|c|c|c|c|c|c|c|c|}
\hline & \multirow[b]{2}{*}{ 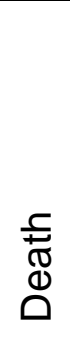 } & & \multicolumn{3}{|c|}{ Systemic } & \multirow[b]{2}{*}{$\begin{array}{l}\text { 응 } \\
\text { 응 } \\
\frac{1}{3} \\
\text { 之 }\end{array}$} & \multirow[b]{2}{*}{ 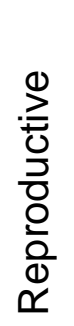 } & \multirow[b]{2}{*}{ 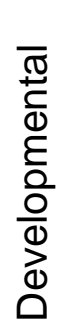 } & \multirow[b]{2}{*}{$\begin{array}{l}\frac{0}{x} \\
0 \\
0 \\
0 \\
0 \\
0 \\
0\end{array}$} & \multirow[b]{2}{*}{$\begin{array}{l}\bar{d} \\
\bar{U} \\
\bar{C} \\
\mathcal{D}\end{array}$} \\
\hline & & $\frac{0}{5}$ & 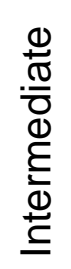 & 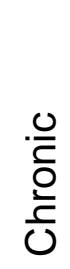 & 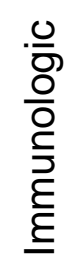 & & & & & \\
\hline \multirow{3}{*}{$\begin{array}{l}\text { Inhalation } \\
\text { Oral } \\
\text { Dermal }\end{array}$} & - & - & - & - & & - & - & - & - & - \\
\hline & - & - & - & - & - & - & & - & & - \\
\hline & & - & - & • & & & & & & \\
\hline \multicolumn{11}{|c|}{ Human } \\
\hline
\end{tabular}

Figure 1.29. Existing Human Information on Health Effects of Trichloroethylene (118) 


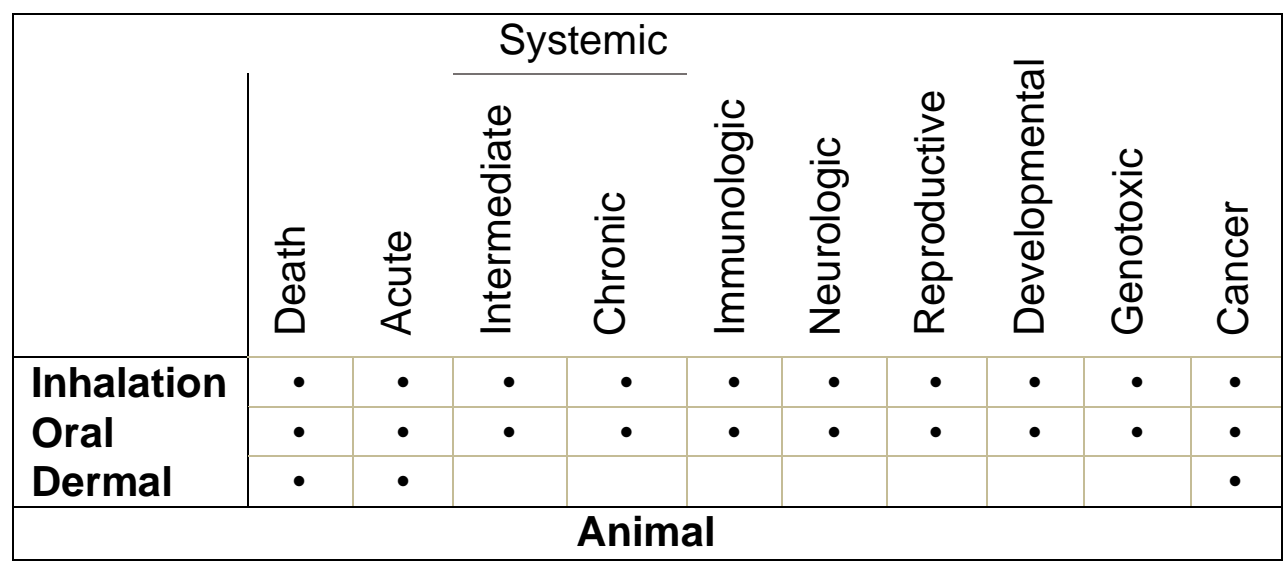

Figure 1.30. Existing Animal Information on Health Effects of Trichloroethylene (115)

\section{Xylene}

Xylene is a colorless, sweet smelling aromatic liquid, which is less dense than, and insoluble in water (119). Xylene occurs naturally in petroleum and coal tar and is synthesized industrially for use as a solvent. Xylene is one of the top 30 chemicals in productive us in the US (120).

Xylene is an important industrial solvent and is used widely in the printing, rubber, and leather industries as well as in cleaning agents, paint thinners, and in paints, varnishes, jet fuel and gasoline. While industrial emission sources have been identified and are regulated, xylene's extensive use in industry and transportation make it quite abundant in the environment and therefore industrial exposure sources are virtually unavoidable (120). In addition to the abundant industrial emission exposures, the general public is exposed to xylene via numerous common-use products. Xylene is present in every-day products, such as gasoline, paint varnish, shellac, rust preventatives, and cigarette smoke (120). 
Acute high levels of exposure to xylene have been reported to cause headaches, lack of muscle coordination, dizziness, confusion, impaired balance, skin irritation, eye irritation, nasal irritation, throat irritation, difficulty in breathing, delayed reaction time, memory difficulties, stomach discomfort, nausea, vomiting, unconsciousness and death, all of which are indicative of affects to the central nervous system. Chronic exposure to xylene may alter liver and kidney function (120). Xylene has been found to be associated with cancer in both mouse and human studies but the current evidence is non-conclusive $(50,121)$. Figures 1.31 and 1.32 provide a summary of existing information on the health effects of xylene (120).

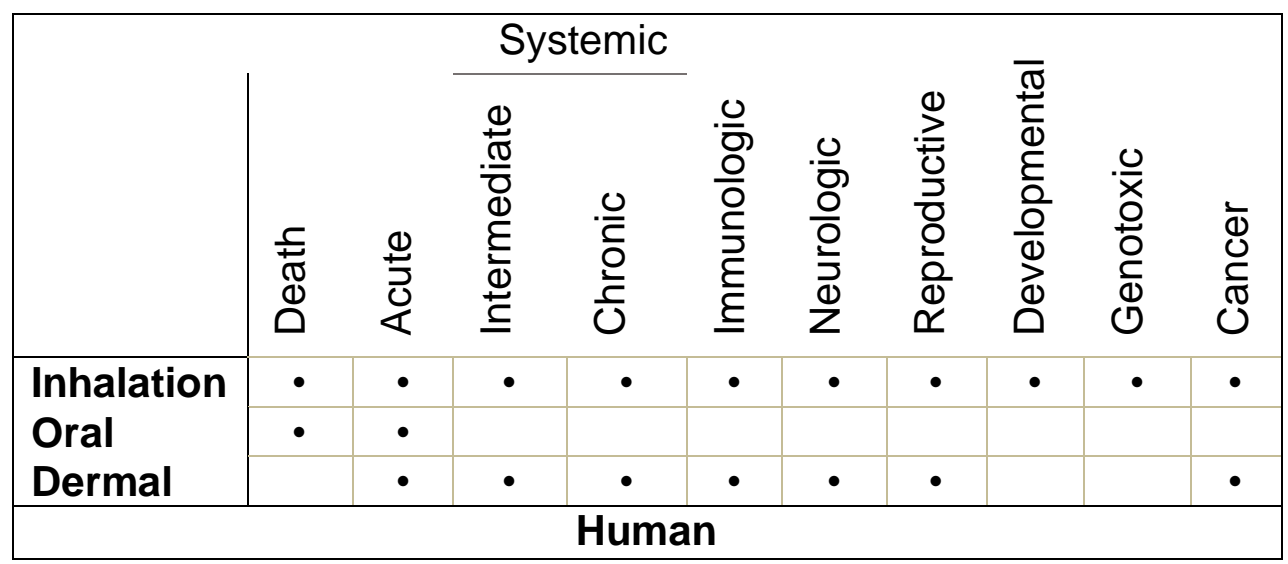

Figure 1.31. Existing Human Information on Health Effects of Xylene (120) 


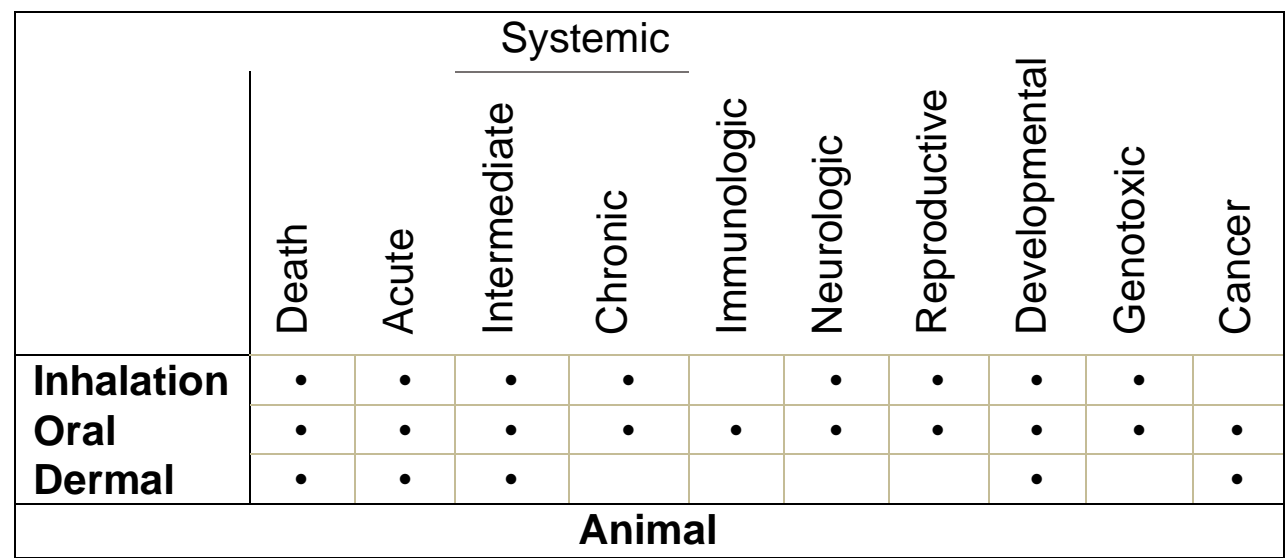

Figure 1.32. Existing Animal Information on Health Effects of Xylene (122)

\section{Multipollutant VOC Effects}

Nearly 200 air toxics have been associated with adverse health effects in occupational, epidemiologic, and/or laboratory studies. All 19 parent VOCs of interest in this investigation have been associated with adverse health effects.

Table 1.1 summarizes the existing information on the health effects of each of the VOC of interest, as reported by Agency for Toxic Substances and Disease Registry (ATSDR). The "H" indicates evidence from a human study what the "a" indicated evidence form an animal study. The letters indicate that one or more studies provide information associated with that particular effect. They do not necessarily imply anything about the quality of the study or studies. As shown in Table 1.1, all the VOCs of interest in this studies have been associated with numerous adverse health effects including cardiovascular and metabolic effects (40). 
Table 1.1. Compilation of Existing Information on Health Effects of the VOCs

Known Health Effects of VOC Exposures

Systemic

\begin{tabular}{|c|c|c|c|c|c|c|c|c|c|c|}
\hline & 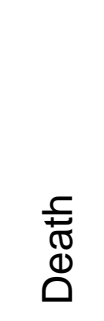 & 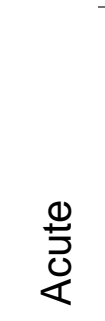 & 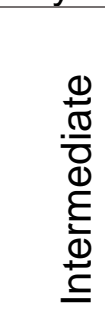 & $\begin{array}{l}\frac{0}{\bar{L}} \\
\frac{0}{\frac{D}{U}}\end{array}$ & $\begin{array}{l}\frac{0}{\bar{O}} \\
\frac{\mathrm{O}}{0} \\
\frac{\mathrm{E}}{\mathrm{E}} \\
\underline{\underline{E}}\end{array}$ & $\begin{array}{l}\frac{0}{2} \\
\frac{0}{0} \\
\frac{0}{2} \\
\frac{0}{2}\end{array}$ & $\begin{array}{l}\stackrel{0}{D} \\
\stackrel{0}{0} \\
\frac{0}{0} \\
\frac{0}{0} \\
\mathbb{0} \\
\simeq\end{array}$ & 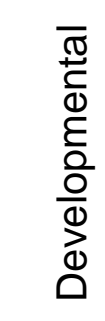 & $\begin{array}{l}\frac{0}{x} \\
0 \\
\frac{0}{0} \\
\frac{0}{0} \\
0\end{array}$ & 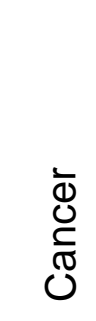 \\
\hline Acrolein & a & $\mathrm{H}, \mathrm{a}$ & $\mathrm{a}$ & a & a & a & a & a & & a \\
\hline Acrylamide & a & $\mathrm{H}, \mathrm{a}$ & a & a & & $\mathrm{H}, \mathrm{a}$ & a & a & a & $\mathrm{H}, \mathrm{a}$ \\
\hline Acrylonitrile & $\mathrm{H}, \mathrm{a}$ & $\mathrm{H}, \mathrm{a}$ & $\mathrm{a}$ & $\mathrm{s}$ & $\mathrm{H}$ & $\mathrm{H}, \mathrm{a}$ & $\mathrm{H}, \mathrm{a}$ & $\mathrm{H}, \mathrm{a}$ & $\mathrm{H}, \mathrm{a}$ & $\mathrm{H}, \mathrm{a}$ \\
\hline Vinyl Chloride & $\mathrm{H}, \mathrm{a}$ & $\mathrm{H}, \mathrm{a}$ & $\mathrm{H}, \mathrm{a}$ & $\mathrm{H}, \mathrm{a}$ & $\mathrm{H}, \mathrm{a}$ & $\mathrm{H}, \mathrm{a}$ & $\mathrm{H}, \mathrm{a}$ & $\mathrm{H}, \mathrm{a}$ & $\mathrm{H}, \mathrm{a}$ & $\mathrm{H}, \mathrm{a}$ \\
\hline Ethylene Oxide & $\mathrm{a}$ & $\mathrm{H}, \mathrm{a}$ & $\mathrm{H}, \mathrm{a}$ & $\mathrm{H}, \mathrm{a}$ & $\mathrm{H}, \mathrm{a}$ & $\mathrm{H}, \mathrm{a}$ & $\mathrm{H}, \mathrm{a}$ & $\mathrm{a}$ & $\mathrm{H}, \mathrm{a}$ & $\mathrm{H}, \mathrm{a}$ \\
\hline Benzene & $\mathrm{H}, \mathrm{a}$ & $\mathrm{H}, \mathrm{a}$ & $\mathrm{H}, \mathrm{a}$ & $\mathrm{H}, \mathrm{a}$ & $\mathrm{H}, \mathrm{a}$ & $\mathrm{H}, \mathrm{a}$ & $\mathrm{H}, \mathrm{a}$ & $\mathrm{H}, \mathrm{a}$ & $\mathrm{H}, \mathrm{a}$ & $\mathrm{H}, \mathrm{a}$ \\
\hline 1-Bromopropane & a & a & $\mathrm{H}, \mathrm{a}$ & a & $\mathrm{a}$ & $\mathrm{a}$ & a & a & a & a \\
\hline 1,3-Butadiene & a & $\mathrm{H}, \mathrm{a}$ & $\mathrm{a}$ & $\mathrm{H}, \mathrm{a}$ & a & $\mathrm{H}, \mathrm{a}$ & $\mathrm{a}$ & a & $\mathrm{H}, \mathrm{a}$ & $\mathrm{H}, \mathrm{a}$ \\
\hline Carbon-disulfide & $\mathrm{H}, \mathrm{a}$ & $\mathrm{H}, \mathrm{a}$ & $\mathrm{H}, \mathrm{a}$ & $\mathrm{H}, \mathrm{a}$ & $\mathrm{H}$ & $\mathrm{H}, \mathrm{a}$ & $\mathrm{H}, \mathrm{a}$ & $\mathrm{H}, \mathrm{a}$ & a & $\mathrm{H}$ \\
\hline Cyanide & $\mathrm{H}, \mathrm{a}$ & $\mathrm{H}, \mathrm{a}$ & a & $\mathrm{H}, \mathrm{a}$ & $\mathrm{a}$ & $\mathrm{H}, \mathrm{a}$ & a & $\mathrm{a}$ & a & \\
\hline Ethylbenzene & $\mathrm{a}$ & $\mathrm{H}, \mathrm{a}$ & $\mathrm{a}$ & $\mathrm{H}$ & & $\mathrm{H}, \mathrm{a}$ & a & a & $\mathrm{H}$ & $\mathrm{H}, \mathrm{a}$ \\
\hline Styrene & a & $\mathrm{H}, \mathrm{a}$ & $\mathrm{H}, \mathrm{a}$ & $\mathrm{H}, \mathrm{a}$ & $\mathrm{H}, \mathrm{a}$ & $\mathrm{H}, \mathrm{a}$ & $\mathrm{H}, \mathrm{a}$ & $\mathrm{H}, \mathrm{a}$ & $\mathrm{H}, \mathrm{a}$ & $\mathrm{H}, \mathrm{a}$ \\
\hline Tetrachloroethylene & $\mathrm{H}, \mathrm{a}$ & $\mathrm{H}, \mathrm{a}$ & $\mathrm{H}, \mathrm{a}$ & $\mathrm{H}, \mathrm{a}$ & a & $\mathrm{H}, \mathrm{a}$ & $\mathrm{H}, \mathrm{a}$ & $\mathrm{H}, \mathrm{a}$ & $\mathrm{H}, \mathrm{a}$ & $\mathrm{H}, \mathrm{a}$ \\
\hline Toluene & $\mathrm{H}, \mathrm{a}$ & $\mathrm{H}, \mathrm{a}$ & $\mathrm{H}, \mathrm{a}$ & $\mathrm{H}, \mathrm{a}$ & $\mathrm{H}, \mathrm{a}$ & $\mathrm{H}, \mathrm{a}$ & $\mathrm{H}, \mathrm{a}$ & $\mathrm{H}, \mathrm{a}$ & $\mathrm{H}, \mathrm{a}$ & $\mathrm{H}, \mathrm{a}$ \\
\hline Trichloroethylene & $\mathrm{H}, \mathrm{a}$ & $\mathrm{H}, \mathrm{a}$ & $\mathrm{H}, \mathrm{a}$ & $\mathrm{H}, \mathrm{a}$ & $\mathrm{H}, \mathrm{a}$ & $\mathrm{H}, \mathrm{a}$ & $\mathrm{H}, \mathrm{a}$ & $\mathrm{H}, \mathrm{a}$ & $\mathrm{H}, \mathrm{a}$ & $\mathrm{H}, \mathrm{a}$ \\
\hline Xylene & $\mathrm{H}, \mathrm{a}$ & $\mathrm{H}, \mathrm{a}$ & $\mathrm{H}, \mathrm{a}$ & $\mathrm{H}, \mathrm{a}$ & $\mathrm{H}, \mathrm{a}$ & $\mathrm{H}, \mathrm{a}$ & $\mathrm{H}, \mathrm{a}$ & $\mathrm{H}, \mathrm{a}$ & $\mathrm{H}, \mathrm{a}$ & $\mathrm{H}$ \\
\hline
\end{tabular}

Exposure to volatile organic compounds is ubiquitous but generally the health effects of the VOCs of interest in this investigation have been studied in the single exposure context, as exemplified in Table 1.1. However, because people are virtually never expose to one single pollutant, studying pollutant mixtures in a more natural exposure spectrum has been greatly desired. Recent advances in both pollutant measurement capabilities and statistical techniques have finally led to the ability to conduct miltipollutant exposure analyses.

Emerging techniques are being developed, which allow environmental 
pollutant mixtures to be studied in a more natural exposure spectrum i.e. mixtures of pollutant. These techniques are referred to as multipollutant models $(123,124)$. These techniques address the multicollinearity of the environmental exposures and provide more robust statistics for addressing or adjusting for multiple comparisons, confounding, and significant covariates.

To date, there have only been a handful of studies that have investigated VOC exposures from a multipollutant prospective $(123,125-128)$. More conventional "two-stage" approaches which estimate exposures and health outcomes first and then combined them into one model have been used to assess the associations between VOCs and non-accidental, cardiovascular, and cancer mortality, as well as fasting glucose, insulin, HOMA-IR, adiponectin, resistin, leptin, and $\mathrm{HbA1c}$. Theses conventional "two-stage" models have been very successful in demonstrating the value of exposure-based research analytic approaches but lack the sophistication of adjusting for or calculating estimates of uncertainty.

There has been a recent recognition of the importance of mixtures investigations in exposure science. While, there have been a handful of multipollutant exposure investigations on VOCs and numerous single-exposuretype investigations of the health effects of VOCs; this is the first investigation to study the combinatory effects of these specific VOCs, to utilize urinary metabolites of VOCs, or to investigate mixtures of these UM-VOCs and their associations with CMS. 


\section{Volatile Organic Compounds and Cardiometabolic Syndrome}

While the carcinogenicity of many of the VOCs of interest is relatively well established, very little is understood about the effect of VOCs on cardiometabolic syndrome. However, various links between aspects of CMS and exposures containing, or associated with, VOCs have been assessed in a variety of research discussed below.

A systematic review of all studies published between 1950 and 2007 of associations between long-term exposure to ambient air pollution and the risks in adults of non-accidental mortality and the incidence and mortality from cancer and cardiovascular and respiratory diseases showed that a $10 \mu \mathrm{g} / \mathrm{m} 3$ increase in longterm exposure to $\mathrm{PM} 2.5$ was associated with a $6 \%$ increased risk of non-accidental mortality, independent of age, gender, and geographic region. The corresponding risk for cardiovascular disease was 12\%-14\% (129).

A large cohort study of 57,053 participants of the Danish Diet, Cancer and Health cohort in the Hospital Discharge Register, assessed exposure to traffic related air pollution, which contains PM and VOCs, showed that traffic related air pollution contributes to non-specified fatal stroke (adjusted Hazard Ratio (HR): 1.79 (1.19-1.49)) (130). A study assessing long-term exposure of $P M 2.5$ found an incidence rate ratio (IRR) for hypertension of $1.48(95 \% \mathrm{Cl}, 0.95-2.31)$ for every 10-mg/m3 increase in PM2.5 and a corresponding IRR for diabetes mellitus of 1.63 $(95 \% \mathrm{Cl}, 0.78-3.44)$ (131). Additionally, a cross-sectional investigation of more

than 8,000 participants recruited form a diabetes-screening study found a significant increase in odds of Type 2 Diabetes (T2DM) for individuals who lived 
within 250 meters of a main roadway $1.44(95 \% \mathrm{Cl}, 1.01-2.05)$ (132). Trafficassociated excess cardiovascular mortality, assessed using single pollutant models has shown significant associations with excess increased risk of CVD mortality with exposure to propylene, benzene, and xylene (133). A large cohort study of more than 58,000 Toronto residents assessed VOC variation within urban areas, and showed associations with CVD mortality and exposure to benzene, hexane, and total hydrocarbon (126). An evaluation of the health effects of 72,000 individuals living within half-a-mile of 258 Superfund sites found excessive rates of stroke and T2DM, these rates were associated with exposure to elevated level of VOCs, such as benzene in drinking water (134). A recent case-control study found an increase in cumulative traffic near the home was associated with a $4 \%$ increase in the odds of acute myocardial infarction (MI) per interquartile range $(95 \% \mathrm{Cl} 2$ $7 \%$ ), and living near a major roadway was associated with a $5 \%$ increase in the odds of acute myocardial infarction $(95 \% \mathrm{Cl}, 3-6 \%)$. Furthermore, a casecrossover study of 2,134 myocardial infarctions, demonstrated a statistically significant association between concentrations of benzene and the onset of $\mathrm{Ml}$; the percent increase in risk of $\mathrm{Ml}$ for a $1 \mu \mathrm{g} / \mathrm{m}_{3}$ increase in ambient benzene concentration in the previous $0,0-1$ and 1 day before the $\mathrm{Ml}$ was $10.4(95 \% \mathrm{Cl}$, 318.2), $10.7(95 \% \mathrm{Cl}, 2.7-19.2)$ and $7.2(95 \% \mathrm{Cl}, 0.3-14.5)$, respectively (135).

Acrolein is a well-established cardiovascular toxicant based on in vitro and in vivo studies (136). It is believed that acrolein generates free oxygen radicals the heart; which decreases endothelial nitric oxide synthase phosphorylation and nitric oxide formation, allowing for the formation of cytoplasmic and nuclear protein 
adducts with myocyte and vascular endothelial cell proteins and causes vasospasm (136). Therefore chronic exposure to acrolein can cause myocyte dysfunction, myocyte necrosis and apoptosis, which ultimately would lead to cardiomyopathy and cardiac failure (136). While these pathways and effects have been well established in both in vitro and in vivo studies, rigorous epidemiologic investigations are needed to establish acrolein's cardiovascular toxicity in humans (136). Acrolein has also been shown to play a role in the disruption of lipid metabolism and neurogenic inflammatory responses $(137,138)$.

In a study of residential proximity to trichloroethylene-emitting sites, CHD risk was over three-fold greater among infants of exposed mothers compared to infants of none-exposed mothers (adjusted OR=3.2 (95\% Cl: 1.2-8.7)) and reports of trichloroethylene-exposures linked to diabetes have been published $(139,140)$.

Occupational exposures to VOCs have also been associated with aspects of CMS. An Italian cohort analysis of 1,658 male workers exposure to vinyl chloride found associations with excess CVD ( $R R=2.25)(141)$. A prospective cohort study of vinyl chloride exposed workers found a marginally significance relative risk for CVD of $1.4(95 \% \mathrm{Cl}: 1.0-1.8)$ for exposed persons as compared to non-exposed persons (142). In an evaluation of 25 liver biopsies of non-obese, highly exposed vinyl chloride workers it was found that the prevalence of steatohepatitis was $80 \%$, indicative of toxicant-associated steatohepatitis, which is characterized by insulin resistance, elevated proinflammatory cytokines and normal liver enzymes (143).

An epidemiologic study published in 2007 showed that a benzene exposed group had higher rates of hypertension than a non-exposed, control group but the 
study did not monitor the concentration and/or extent of the benzene exposure (144). An age- and sex- matched case-control study of 345 petrochemical factory workers found increased odds of arterial hypertension with both benzene- and xylene- exposure $2.44(95 \% \mathrm{Cl}, 1.24-4.85), 2.00$ (95\% Cl, 1.11-3.61) respectively. Pathologic changes on electrocardiogram were also observed for both benzene and xylene-exposed subjects $2.34(95 \% \mathrm{Cl}, 0.99-5.63), 2.75(95 \% \mathrm{Cl}, 1.28-5.97)$ respectively (145).

Excess mortality from ischemic heart disease in $\mathrm{N}, \mathrm{N}$-dimethylformamideexposed workers at an acrylonitrile fiber plant was observed. The investigation looked at workers in a plant exposed to N, N-dimethylformamide between 1950 and 1982 (146). Among the plant workers there were 62 deaths due to ischemic heart disease, which was significantly higher than expected (40.3 expected for company) ( $p$-value $<0.01$ ); However, the seemingly increased rate was not significant when compared with the state rates (146). Similarly a second group of 1,329 employees at a plant where workers were potentially exposed to both $\mathrm{N}, \mathrm{N}$-dimethylformamide and acrylonitrile observed a significantly higher number of deaths among workers; 65 deaths among the plant workers, when 48.3 was expected for company ( $p$ value< 0.05$)$ (146). However, again the observed number of deaths was not significantly higher than expected when compared to state or national rates (146). A small study in which electrocardiographic (ECG) monitoring was performed on workers at a synthetic leather plant where N, N-dimethylformamide was used found isolated ventricular premature beats after 2 hours of work, without pathological alterations (147). Reports of ECG changes, and cardiac disturbances, 
including tachycardia and palpitations have also been reported in workers exposed to N, N-dimethylformamide (148-150).

Cyanide exposure has been linked to diabetes through consumption of cassava and cassava workers exposed to hydrogen cyanide have been shown to have significantly higher triglycerides and atherogenic plasma; acute cyanide exposures have also demonstrated sever cardiovascular disruption (151-153).

One report of chronic carbon disulfide exposure causing severe vascular disease has been published and a critical review of epidemiologic literature conducted in 2002 found consistent reports of associations with carbon disulfide exposure and dyslipidemia $(154,155)$. Carbon disulfide poisoning has also been shown to significantly increase the risk of metabolic syndrome in Korean viscose rayon factory workers (prevalence ratio=1.57 (95\% Cl: 1.25-1.98)) (156).

1,3-Butadiene has well established laboratory and epidemiologic associations with cardiovascular disease as well as atherosclerosis and cardiac injury (157). A longitudinal epidemiologic investigation found significant associations between occupational exposure to 1,3-butadiene and CVD (157). In an analysis of mortality data, collected from more than 12,000 male workers at eight 1,3-butadiene-styrene polymer manufacturing plants in the U.S. and Canada from 1943 to 1982 , a significant increase in the standardized mortality ratio for arteriosclerotic heart disease $(\mathrm{SMR}=1.48)$ was found among black maintenance workers, as compared to the levels expected from those for the general population (158). 1,3-Butadiene has also been shown to significantly increase atherosclerotic plaque size in multiple in vivo and in vitro studies $(157,159,160)$. Taken together, 
these studies suggest that VOC exposure might be an important contributing factor to CMS risk.

\section{Cardiometabolic Syndrome}

Many studies have demonstrated the mounting problem posed by cardiometabolic syndrome both within the United States and worldwide (161-169). Given the associated monetary costs (U.S.: 80 billion dollars annually) and dramatically effected quality and longevity of life for those affected it is important to understand the driving factors of CMS, areas for future research, and how public health, clinical, and research communities may address this growing issue in our society (170).

Definition of Cardiometabolic Syndrome

Cardiometabolic syndrome (CMS) is a combination of maladaptive cardiovascular, renal, metabolic, prothrombotic, and inflammatory abnormalities that independently and interdependently dramatically increases an individual's cardiovascular disease (CVD) risk (1). The increased CVD risk associated with CMS has been shown to be a significant predictor of premature and severe CVD, coronary heart disease, myocardial infarction, and stroke and therefore highly associated with increased CVD morbidity and mortality (1).

There is no universally accepted definition of CMS. To date at least five organizations have proposed clinical criteria for diagnosing CMS (24). The most common characteristics for defining CMS are abdominal obesity (high body mass index (BMI), waist circumference, waist-to-hip circumference ratio (WHR)), insulin resistant glucose metabolism (hyperinsulinemia, impaired fasting glucose, 
impaired glucose tolerance, type II diabetes (T2DM)), dyslipidemia (high serum triglyceride and low serum high-density lipoprotein-cholesterol concentrations), and increased blood pressure. The criteria proposed by the World Health Organization (WHO) are the most widely accepted criteria, and will be utilized as the criteria of interest in this paper (Table 1.2) (24).

The WHO defines CMS as the presence of dysglycemia, which is abnormal blood glucose levels (measured by impaired fasting glucose (IFG)/ impaired glucose tolerance (IGT)/ T2DM)), plus 2 or more of the following criteria: visceral obesity (measured as; men: $>0.90 \mathrm{WHR}$ or BMI $\geq 30 \mathrm{~kg} / \mathrm{m} 2$, women: $>0.85 \mathrm{WHR}$ or $\mathrm{BMI} \geq 30 \mathrm{~kg} / \mathrm{m} 2$ ), dyslipidemia (classified by elevated triglycerides $(\geq 1.7 \mathrm{mmol} / \mathrm{L})$ ) and low HDL-cholesterol (defined as men: $<0.9 \mathrm{mmol} / \mathrm{L}$, women: $<1.0 \mathrm{mmol} / \mathrm{L}$ ), hypertension ( $\geq 140 / 90 \mathrm{~mm} \mathrm{Hg}$ ), and/or the presence of Microalbuminuria (defined as urinary excretion $\geq 20 \geq 20 \mu \mathrm{g} / \mathrm{min}$ or Albumin:Creatinine ratio $\geq 30 \mathrm{mg} / \mathrm{g}$ (24).

Table 1.2. World Health Organization Definition of Cardiometabolic Syndrome

\begin{tabular}{|c|c|}
\hline Condition & 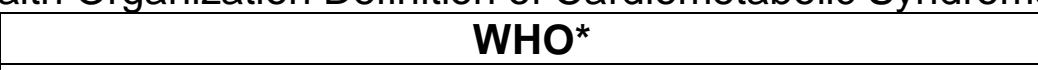 \\
\hline 1. Dysglycemia & IFG/IGT/T2DM \\
\hline \multicolumn{2}{|l|}{ 2. Visceral obesity } \\
\hline Men & $>0.90 \mathrm{WHR}$ and/or $\mathrm{BMI}>30 \mathrm{~kg} / \mathrm{m} 2$ \\
\hline Women & $>0.85 \mathrm{WHR}$ and/or $\mathrm{BMI}>30 \mathrm{~kg} / \mathrm{m} 2$ \\
\hline 3. Triglycerides & $\geq 1.7 \mathrm{mmol} / \mathrm{L}(150 \mathrm{mg} / \mathrm{dL})$ \\
\hline \multicolumn{2}{|l|}{ 4. HDL-cholesterol } \\
\hline Men & $<0.9 \mathrm{mmol} / \mathrm{L}(35 \mathrm{mg} / \mathrm{dL})$ \\
\hline Women & $<1.0 \mathrm{mmol} / \mathrm{L}(39 \mathrm{mg} / \mathrm{dL})$ \\
\hline 5. Hypertension & $\geq 140 / 90 \mathrm{~mm} \mathrm{Hg}$ \\
\hline 6. Microalbuminuria & $\begin{array}{l}\text { Urinary albumin excretion } \geq 20 \mu \mathrm{g} / \mathrm{min} \text { or } \\
\text { Albumin:Creatinine ratio } \geq 30 \mathrm{mg} / \mathrm{g}\end{array}$ \\
\hline $\begin{array}{l}\text { IFG=impaired fastins } \\
2 \text { diabetes mellitus; }\end{array}$ & $\begin{array}{l}\text { glucose; IGT = impaired glucose tolerance; T2DM=type } \\
\text { NHR=waist-to-hip circumference ratio }\end{array}$ \\
\hline
\end{tabular}




\section{Dysglycemia}

Dysglycemia is a broad term used in reference to any abnormalities in blood glucose levels (171). Dysglycemia is often tested with the impaired fasting glucose (IFG) test and impaired glucose tolerance (IGT) test $(24,171)$. IFG is defined as an elevated fasting plasma glucose (FPG) concentration ( $\geq 100$ and $<126 \mathrm{mg} / \mathrm{dl}$ ) and IGT is defined as an elevated two hour plasma glucose concentration ( $\geq 140$ and $<200 \mathrm{mg} / \mathrm{dl}$ ) after a 75 gram glucose load on the oral glucose tolerance test in the presence of an FPG concentration $<126 \mathrm{mg} / \mathrm{dl}$ (172). Both the IFG and IGT are early indicators of abnormal glucose metabolic abnormalities that precede diabetes (172). Diabetes is a group of diseases marked by high levels of blood glucose resulting from insulin dysfunction. Current estimates indicate that up to $70 \%$ of individuals with IFG or IGT in a pre-diabetic state will eventually develop T2DM, (172) hence the WHO criteria of displaying an abnormal IFG or IGT test or having been diagnosed the T2DM (172). A diagnosis of T2DM encompasses they presence of dysglycemia (171).

There are two main classes of diabetes: type I and type II diabetes: type I diabetes, which accounts for approximately $5 \%$ of all adult diabetes, is due to a limited or eliminated production of insulin by the beta cells in the pancreas (173). Type II diabetes, which accounts for $95 \%$ of diagnosed diabetes cases, is a result of insulin resistance, which can lead to a gradual loss of the ability of the beta cells in the pancreas to produce enough insulin as the insulin demand from malfunctioning cells rises (174). There are other types of diabetes that result from other medical complication such as surgery, medications, infections, pancreatic 
disease, pregnancy, or other illnesses, but these forms of diabetes are rare and only account for $1-5 \%$ of all diagnosed diabetes cases $(173,174)$.

Other common indicators of dysglycemia are the hemoglobin A1c (HbA1c) test and the homeostasis model assessment of insulin resistance (HOMA-IR) score. $\mathrm{HbA} 1 \mathrm{c}$ is used to measure long-term (2-3 months) blood glucose stability (175). A recent study suggests that an $\mathrm{HbA} 1 \mathrm{C} \geq 6.0 \%$ approximately doubles the rate of progression to diabetes in the IFG/IGT population (176). The "homeostasis model assessment of insulin resistance' (HOMA-IR) is an insulin resistance and sensitivity indices. An insulin resistance score is calculated using the HOMA-IR indices and is calculated by multiplying an individual's fasting plasma glucose $(\mathrm{mmol} / \mathrm{l})$ by his/her fasting serum insulin ( $\mathrm{mU} / \mathrm{l})$ and then dividing that by 22.5 (177).

\section{Visceral Obesity}

Visceral obesity is associated with insulin resistance; however, causation has not been established (i.e. it is not known if visceral obesity is a causal factor of insulin resistance or if visceral obesity and insulin resistance are simply associated). While visceral fat is only a small percentage of total body fat (10-15\%), it has been postulated to play a strong role in insulin resistance due to direct portal vein entry of fatty acids during lipolysis, which delivers fatty acids directly to the liver (178).

Measures of visceral obesity that have been established as predictors of CVD and CMS are body-mass index (men: BMI $\geq 30 \mathrm{~kg} / \mathrm{m} 2$, women: BMI $\geq 30$ $\mathrm{kg} / \mathrm{m} 2$ ), waist-to-hip circumference ratio (WHR) (men: WHR >0.90, women: WHR $>0.85$ ), and waist circumference (WC) (men: $W C \geq 94 \mathrm{~cm}$ or $>102 \mathrm{~cm}$, women: WC 
$\geq 80 \mathrm{~cm}$ or $>88 \mathrm{~cm})(179,180)$. It is recognized that abdominal fat mass, also referred to as central visceral fat, can also be an important consideration in identifying individuals with increased risk of obesity-related morbidity and mortality.

Waist-to-hip ratio is an index of both subcutaneous and intra-abdominal adipose tissue distribution and has long been shown to be a good, noninvasive, anthropometric indicator of abnormal adiposity and increased disease risk. In 1997 the WHO Expert Consultation on Obesity convened to discuss the efficacy of WHR and WC as additional measures in assessing risk. In 2000 the WHO suggested waist-to-hip ratio and waist circumference as additional measures to BMI, to aid in discerning body fat distribution.

A longitudinal cohort study, published by Larsson et al. in 1984, showed that waist-to-hip ratio in men was better at distinguishing increased risk of myocardial infarction, stroke, and premature death, than BMI (181). In women BMI was more associated with increased risk; however the study found that WHR was a stronger independent indicator of risk than BMI (181). Additionally, a recent study found that WC is a better indicator of CVD risk than BMI and proposed WC cutpoint values that are more sensitive than the BMI cut-points for predicting CVD risk (179).

While waist-to-hip ratio and waist circumference have proven to be a valuable tool in assessing risks associated with abnormal fat mass; the cut-points for diagnosing abnormal fat mass have been highly variable. The variability in the cut-point definitions is a result of the fact that WHR and WC cut-points are dependent on population characteristics (sex, age, ethnicity) and the outcome of 
interest, whether that be increased risk of CVD, diabetes, or overall mortality. The 2008 Waist Circumference and Waist-Hip Ratio report of a WHO Expert Consultation summarizes the complexities of defining cut-points and the existing evidence to support recommended cut-point action levels (182). While populationspecific cut-points may be useful, at the present time the WHO consultation reported too many unresolved issues in defining population-specific cut-points and opted for simple and universally applicable cut-points. The WHO consultation agreed that BMI, WHR, and WC are all predictive of the risk of chronic disease and may therefore be used independently or in conjunction with BMI $(182,183)$. The report indicates that a $\mathrm{BMI}$ of 30 is comparable in increased disease risk to a waist circumference of $>94 \mathrm{~cm}$ for men and $>80 \mathrm{~cm}$ for women and a substantially increased risk at $>102 \mathrm{~cm}$ for men and $>88 \mathrm{~cm}$ for women and a waist-to-hip ratio of cut-point of 0.91 for both me and women (Table1.3) $(182,183)$. The designations "increased" risk and "substantially increased" risk, referenced to in Table 1.3 and designated in the WHO report are based on the increased relative risks observed in a Netherlands study of a random sample of 2,183 men and 2,698 women aged 20-59 years. The Netherlands study found that compared with individuals below the identified waist measurement cut-points, the age and lifestyle adjusted odds ratios for having at least one cardiovascular risk factor (total cholesterol $>$ or $=6.5 \mathrm{mmol} / \mathrm{l}$, high density lipoprotein cholesterol $<$ or $=0.9 \mathrm{mmol} / \mathrm{l}$, systolic blood pressure $>$ or $=160 \mathrm{~mm} \mathrm{Hg}$, diastolic blood pressure $>$ or $=95 \mathrm{~mm}$ $\mathrm{Hg})$ was $2.2(95 \% \mathrm{Cl}, 1.8-2.8)$ in men with a waist measurement of $94-102 \mathrm{~cm}$ and $1.6(95 \% \mathrm{Cl}, 1.3-2.1)$ in women with a waist measurement of $80-88 \mathrm{~cm}$. In men 
and women with larger waist measurements these age and lifestyle adjusted odds ratios were $4.6(95 \% \mathrm{Cl}, 3.5-6.0)$ and $2.6(95 \% \mathrm{Cl}, 2.0-3.2)$ respectively (184).

The International Diabetes Federation recommends WC cut-points of $>94 \mathrm{~cm}$ for men and $>80 \mathrm{~cm}$ women of European descent, and $>90 \mathrm{~cm}$ for men and $>80 \mathrm{~cm}$ for women of South Asian, Chinese and Japanese descent $(180,185,186)$. The recommendations made by the United States National Cholesterol Education Program (NCEP) of the NIH's National Heart, Lung, and Blood Institute are a single set of sex-specific cut-point of $>102 \mathrm{~cm}$ for men and $>88 \mathrm{~cm}$ for women (122).

It is recommended that WC or WHR be used in conjunction with $\mathrm{BMI}$, as increased waist circumference can also be a marker of increased risk even in person of normal weight (187). Furthermore, increases in WC have been shown to be useful predictors of changes in CVD risk (188).

Table 1.3. World Health Organization Cut-Points and Risk of Metabolic Complications $(182,184)$

\begin{tabular}{|l|l|l|}
\hline Indicator & Cut-point & $\begin{array}{l}\text { Risk of Metabolic } \\
\text { Complications }\end{array}$ \\
\hline Waist circumference & $>94 \mathrm{~cm}(\mathrm{M}) ;>80 \mathrm{~cm}(\mathrm{~W})$ & Increased \\
\hline Waist circumference & $>102 \mathrm{~cm}(\mathrm{M}) ;>88 \mathrm{~cm}(\mathrm{~W})$ & Substantially increased \\
\hline Waist-hip ratio & $\geq 0.90 \mathrm{~cm}(\mathrm{M}) ; \geq 0.85 \mathrm{~cm}(\mathrm{~W})$ & Substantially increased \\
\hline Body Mass Index & $>30.0 \mathrm{~kg} / \mathrm{m} 2$ & Substantially increased \\
\hline
\end{tabular}

${ }^{*} \mathrm{M}$, men; W, women

\section{Dyslipidemia}

Atherosclerotic cardiovascular disease (CVD) risk and diabetes mellitus risk are significantly increased in patients with CMS. The increased risk is due to insulin resistance and atherogenic dyslipidemia (189). Atherogenic dyslipidemia is defined as the presence of low high-density lipoprotein (HDL) cholesterol and high triglyceride levels $(79,190)$. 
Alterations in free fatty acid metabolism are theorized to have a major role in the pathogenesis of dysglycemia and dyslipidemia associated with cardiometabolic syndrome (24). It is thought that increased plasma free fatty acids may impair the ability of insulin to stimulate muscle glucose uptake and suppress hepatic glucose production (24). Increased free fatty acid in the liver increases very low-density lipoprotein (vLDL) production and plasma triglyceride concentrations (24). The increase in plasma triglyceride concentration increases the rate of vLDL to $\mathrm{HDL}$ transfer, thereby increasing the overall HDL clearance and decreasing plasma HDL concentrations (24). Plasma HDL cholesterol concentrations are tested with a simple blood test. The WHO criteria for HDL-cholesterol as a risk factor for cardiometabolic syndrome is $<0.9 \mathrm{mmol} / \mathrm{L}$, for men and $<1.0 \mathrm{mmol} / \mathrm{L}$ for women (1). The WHO criteria for increased plasma triglycerides is, $\geq 1.7 \mathrm{mmol} / \mathrm{L}$, which has been shown to be a significant predictor for increased risk of developing cardiometabolic syndrome and is associated with increased CVD morbidity and mortality (24).

\section{Hypertension}

Insulin resistance and hypertension have a long-standing, well-established relationship and the mounting evidence of a linear relationship between blood pressure and cardiovascular mortality and interaction between hypertension and diabetes support a strong association between hypertension and several vascular and metabolic abnormalities that are components of the cardiometabolic syndrome. A recent report stated more than $80 \%$ of hypertensive patients present with at least one associated metabolic risk factor $(58,88)$. 
In clinical terms, hypertension is recognized as having elevated systolic and/or diastolic blood pressure. Each hypertensive stage definition and criteria are included below (51):

- $\quad$ Stage one hypertension: Systolic blood pressure from 140-159 mm $\mathrm{Hg}$ or a diastolic blood pressure of $90-99 \mathrm{~mm} \mathrm{Hg}(51)$.

- Stage two hypertension: Systolic blood pressure over $160 \mathrm{~mm} \mathrm{Hg}$ or a diastolic pressure over $100 \mathrm{~mm} \mathrm{Hg} \mathrm{(51).}$

Individuals who exhibit resistance to insulin have a decreased or even a complete loss of the vasodilatory effects of insulin (which in normal functioning individual, insulin acts as a vasodilator) (24). Individuals who have a decreased vasodilatory effect often maintain sodium reabsorption, leading to a

significantly increased blood pressure (191). It has been shown that $25-47 \%$ of patients with hypertension are also insulin resistant and/or have IGT, providing epidemiologic support that hypertension and insulin resistance may be causally related (1).

\section{Microalbuminuria}

Microalbuminuria has been associated with visceral obesity, dyslipidemia, and dysglycemia (1). Clinically, microalbuminuria is recognized as urinary excretion of albumin of 20 to $200 \mu \mathrm{g} / \mathrm{min}(30-300 \mathrm{mg} / \mathrm{d}$ ). Microalbuminuria is used as a predictor of CVD and premature CVD mortality. According to a recently published article in Diabetes Care, which investigated 3,606 subjects with and without metabolic syndrome, researchers found that microalbuminuria is the most strongly associated cardiometabolic syndrome criteria among all subjects who 
died from cardiovascular disease (relative risk: $2.80(95 \% \mathrm{Cl}, 1.62-4.83))(1,192)$. Despite the very strong association between microalbuminuria with CVD the mechanism is still not understood (1).

Cardiometabolic Syndrome Descriptive Epidemiology

Cardiometabolic syndrome (CMS) has become a major problem in the United States and worldwide. An analysis of data from the National Health and Nutrition Examination Survey (NHANES III) (1988-1994), found the age-adjusted rate of CMS in the United States was approximately $24 \%$ of the adult population (non-institutionalized U.S. population over the age of 20$)(54,118)$. This study found that among the U.S. male population, white and Mexican Americans had the highest prevalence of abdominal obesity, hypertriglyceridemia and low HDL levels, while African American males had the highest hypertension rates (age-adjusted) (118). Among the female U.S. population, Mexican Americans and African Americans had the highest rates of obesity (age-adjusted), while Mexican Americans had the highest rates of hypertriglyceridemia and low HDL levels, and hyperglycemia (118). The study found that prevalence of CMS differed by race with the highest prevalence being found in the Mexican American (31.9\%) population, and the lowest rates in the White (23.8\%) and African American (21.6\%) populations (54). The study also suggested a linear relationship exists between CMS and age with a prevalence of approximately $7 \%$ for $20-29$ year olds and $45 \%$ of individuals over the age of 60 (54). Notably, this study also suggests that CMS prevalence is steadily increasing (54). It has also been shown that rates have of CMS have been steadily increasing the U.S. A study published in 2004 
compared CMS prevalence from NHANES 1988-1994 with NHANES 1999-2000 and found that age-adjusted prevalence was 24.1 and $27.0 \%$ respectively (101). Other analyses of more recent NHANES data have published adult ( $\geq 20$ years of age) U.S. age-adjusted prevalence rates ranging from $35-39 \%(46,74,109)$. It has also been shown that the prevalence of CMS dramatically increases with age. One study found that the prevalence increased from $6.7 \%$ among participants aged 20 through 29 years to $43.5 \%$ and $42.0 \%$ for participants aged 60 through 69 years and aged at least 70 years, respectively (118).

It is very common that individuals who have been diagnosed with one cardiometabolic syndrome risk factor, such as IFG and IGT, will present with or develop multiple associated CVD and CMS risk factors. A recent analysis of overweight subjects (BMI $\geq 25$ ) aged 45 to 74 years found that $17.1 \%$ of had IGT, $11.9 \%$ had IFG, $22.6 \%$ had prediabetes, and that $5.6 \%$ of subjects had both IGT and IFG (193). The study determined 12 million people age 45-74 in the U.S. have dysglycemia that will likely evolve into T2DM (1). These individuals along with other insulin resistant individuals have been shown to have a nearly threefold increase of CVD and stroke incidence (1). Often individuals with insulin resistance and dysglycemia manifest other components of CMS, which amplify the CVD morbidity and mortality (163).

Cardiometabolic risk factors are increasingly acquired at alarming rates in the United States. A study of NHANES data showed a substantial increase of more than $3 \%$ in the median childhood BMI (ages 6-17) from two different study periods (1963-1970 and 1988-1994) (113). It has been estimated that by 2030 the rate of 
diabetes in the U.S. will have risen from 17.7 million in 2000 to nearly 30.3 million (5).

Currently T2DM afflicts $9.3 \%$ of the U.S. population, (29.1 million people), and is responsible for over 73,000 deaths per year (174). The prevalence of IFG and IGT in the U.S. are currently estimated to be approximately $26 \%$ and $15 \%$ respectively, but expected to steadily increase (172). Estimates of adult (age 20 to 74 years) dyslipidemia rates in the U.S. are approximately $36 \%$, while the prevalence of hypertension in the U.S. is estimated at $24-29 \%$ of the adult population ( $\geq 20$ years) $(70,96,164,165,194,195)$. The prevalence of creatinine normalized microalbuminuria was found to be $6.1 \%$ in the U.S. male population and $9.7 \%$ in the U.S. female population in an analysis of NHANES participants 20 years of age and older (196).

\section{Risk Factors, Potential Covariates and Effect Modifiers}

Cardiovascular and diabetic diseases encompassed in the definition of cardiometabolic syndrome are the leading and preventable causes of death worldwide (161). Currently, the leading preventable risk factor associated with these diseases is tobacco smoke exposure (161). While tobacco smoke exposure has declined over the past half-century, it is estimated that over 34 million Americans still use tobacco $(161,197)$. Tobacco smoke exposure is cited as the number one preventable risk factor of CVD, CMS, and metabolic syndrome and is included in nearly all publications and analysis of these conditions (161). Increased odds of metabolic syndrome have been observed in currently smoking individuals as compared to individuals self-reporting they are nonsmokers (198). An analysis 
of individuals included in the National Health and Nutrition Examinations 19881994 found that currently smoking men have a 1.5 times greater odds $(95 \% \mathrm{Cl}$, 1.1-2.2) of having CMS compared with men who report never smoking and currently smoking women have a 1.8 times greater odds $(95 \% \mathrm{Cl}, 1.2-2.6)$ of having CMS compared with women who report never smoking (198). It is currently hypothesized that cigarette smoke is linked to cardiometabolic syndrome through its effect on insulin resistance (198).

Cardiometabolic syndrome is thought to develop as a result of both genetic factors and acquired/environmental factors. It is hypothesized that genetic factors predispose individuals to magnified effects of environmental exposures, which in conjunction amplify the effects if cardiovascular and metabolic adversities (199). It is unutterably difficult to parse out all the covariates that could be considered for this analysis, as many may not be known. Of the immense number of potential covariates that could be considered, several of those deemed most significant are discussed below.

Each of the factors associated with developing cardiometabolic syndrome have no shortage of covariates associated with their onset. Covariates that have been assessed previously for their association with increased risk of cardiometabolic syndrome are: socioeconomic status, sex, age, ethnicity, alcohol consumption patterns, physical activity, BMI, dietary habits, and for women menopausal status $(198,200,201)$. A recent analysis of adults ( $\geq 20$ years old) who met the criteria for metabolic syndrome among NHANES data estimated the prevalence of metabolic syndrome to be similar among men and women, with a 
slightly elevated relative risk in postmenopausal versus premenopausal women (198). The risk of cardiometabolic syndrome and related complications increases with age. Evidence suggests that peak prevalence for the onset of cardiometabolic syndrome is around 60 years of age (198). Also, rates of cardiometabolic syndrome are highly stratified by ethnicity with black men having the lowest rates (13.9\%) and Mexican Americans women having the highest rates (27.2\%) (202). $\mathrm{BMI}$ is also a very highly associated factor for the development of cardiometabolic syndrome (198). In an NHANES analysis only $6 \%$ of normal weight adults meet the diagnostic criteria for metabolic syndrome, while $60 \%$ of moderately obese individuals (BMI $\geq 30$ ) meet the diagnostic criteria for metabolic syndrome (198). Numerous studies have linked socioeconomic status and education level with various cardiovascular risk factors including impaired glucose tolerance, diabetes, obesity, hypertension, and smoking rates $(79,169,198,203,204)$.

Epidemiologically, low to moderate consumption of alcohol has been shown to decrease risk of cardiovascular disease possibly due to the beneficial alteration of HDL and blood pressure (202). The same decreased risk associated with low to moderate alcohol consumption has been shown to reduce metabolic syndrome potentially due to the same beneficial alteration of HDL and blood pressure (202). It has been shown that a diet high in carbohydrates increases the risk of cardiometabolic syndrome (198).

\section{Comorbidities}

There are a large number of comorbidities associated with CMS. Diabetes, one of the diseases defined within CMS affects many parts of the body. Diabetes 
is associated with an increased risk of heart disease, stroke, blindness, kidney failure, microvascular disease, and lower-limb amputation (189). People with diabetes also have an increased risk of developing, non-alcoholic fatty liver disease, periodontal (gum) disease, hearing loss, erectile dysfunction, depression, and complications during pregnancy (174). A recent study conducted in Spain of more than 1,800 hypertensive patients, found that $50-70 \%$ and patients with metabolic syndrome also had hypertension (189). CMS often progresses to CVD. CMS and CVD share many comorbidities and risk factors. Cancer has recently been identified as a comorbidity of CVD. This is a recent finding but it is reasonable to assert that if CVD and cancer are comorbid conditions and CMS is often a precursor to CVD than CMS and cancer may also be comorbid conditions, especially given the common shared risk factors (poor diet, smoking, obesity, and diabetes) (205).

\section{Summary}

The relative importance of environmental exposures such as volatile organic compounds is one of the paramount public health priorities of our time, yet is presently unstudied and effectively unknown. CMS, a collection of dysfunctional cardiovascular and metabolic functions, is associated with an abundance of comorbidities and dramatically increases ones risk of CVD morbidity and mortality. The consequences of CMS are severe both socially and economically. A number of studies have found associations between numerous health effects, including both cardiovascular and metabolic outcomes and individual VOCs; however, to

date there have been no investigations of the associations between VOCs and 
CMS at a population level, nor have there been any investigations of the combinatory effects of the VOCs of interest with CMS. Exposures relating to or associated with VOCs have previously been significantly associated with hypertension, atherosclerosis, type II diabetes, stroke, and CVD mortality. However, the specific components of the broad classification-exposures (e.g., PM or "traffic-associated" exposure) assessed in these investigations that may be primarily responsible for these adverse health effects have not been identified. This study is a novel investigation of nationally representative individual-level data, which will interrogate associations between CMS and VOCs, from both an individual VOC exposure perspective and a multipollutant exposure perspective. The findings from this investigation are relevant and important for the development of public health interventions and policies surrounding modifiable environmental pollution exposures and their potential role in CMS, a condition which ultimately greatly increases one's risk of the deadliest non-communicable disease in the world, cardiovascular disease. 


\title{
CHAPTER TWO
}

\author{
METHODS
}

\section{Study Design}

Source of Study Population- National Health and Nutrition Examination Survey (NHANES)

The National Health and Nutrition Examination Survey (NHANES) is a cross-sectional, nationally representative survey of the non-institutionalized civilian population in the United States and is designed to assess the health and nutritional status of adults and children in the country (206). NHANES is conducted by the National Center for Health Statistics (NCHS; Centers for Disease Control and Prevention (CDC), Atlanta, $\mathrm{GA}$ ), which is part of the CDC and is responsible for producing vital and health statistics for the nation.

The National Health and Nutrition Examination Survey began in 1960, as the National Health Examination Survey. In 1971 a nutritional assessment and an environmental component were added to the survey thus assuming the name The National Health and Nutrition Examination Survey. NHANES I was conducted from 1971 to 1975 and a subset of this phase of NHANES, consisting of individuals over the age of 25, which became a longitudinal follow-up study through1992 known 
as the NHANES I Epidemiological Follow-up Survey (NHEFS). NHANES II was conducted from 1996 to 1980, NHANES III was conducted from 1988 to 1994, and in 1999 NHANES became a continuous annual survey, with data released to the public in 2-year intervals. The continuous NHANES assessment utilizes multistage stratified probability sampling of approximately 5,000 persons throughout fifteen counties across the U.S. each year. The multistage stratified probability sampling is based on selected counties, blocks, households, and individuals. Over sampling of certain vulnerable population, such as, non-Hispanic black individuals, Hispanic individuals, low-income white individuals, and older adults, 80 and older, is necessary to ensure estimates of these groups are adequately representative. The NCHS Research Ethics Review Board approves the NHANES study protocols and all participants provide written informed consent.

NHANES is the only national survey that collects extensive health and nutrition information from face-to-face interviews and medical examinations. The interview portion of NHANES includes demographic, socioeconomic, dietary, and health-related questions, while the examination component consists of medical, dental, and physiological measurements, as well as laboratory tests.

NHANES is designed to produce statistics for a nationally representative sample and is a complex, continuous survey. Therefore, design-based analytic procedures must be explicitly considered and features of the survey design, such as differential selection probabilities and geographic clustering considerations need be considered in the analysis. The product of a properly weighted NHANES analysis results in statistics that are generalizable to the national residential civilian 
noninstitutionalized U.S. population.

The primary design-based components of the NHANES survey that must be accounted for when conducting an analysis to generate nationally representative results are the clustering variable or the primary sampling unit (PSU), the weight variable or the two-year sample weight, and the stratification variable or stratum. The primary sampling units are counties or small groups of contiguous counties across the US. From the approximately 15 counties or PSUs visited each year a block containing a cluster of households is selected and specific addresses from the cluster is then chosen (207). Primary sampling units always include all four regions of the U.S. as well as metropolitan and nonmetropolitan representatives. Each selected address is contacted by a field interviewer who conducts a screener to enumerate the household and to determine the eligibility of individuals in the household for the survey. Eligibility is based on each individual in the households age, sex, race/ethnicity and income. Based on the screening information collected, a computer algorithm randomly selected one, some, all, or none of the individuals in the household to participate in the survey. On average 12,000 individuals are selected to participate, 10,500 individuals agree to complete the household survey, and 10,000 persons participate in data collection in the mobile exam center (207). A weight variable is assigned to every person and is considered a measure of the number of people in the U.S. represented by that particular sample participant. For example, if a man in the sample represents 12,000 men in his race and ethnicity-income-age category, then his sample weight to begin with is 12,000 . The weighting variable takes into 
account the differential probability of selection for the individual domains, nonresponse to survey instruments, and differences between the final sample and the total population (207).

The first step in assigning a sample weight is to compensate for the unequal probability of selection, given that some groups are oversampled. Next, the weighting variable is adjusted for participant nonresponse and finally the sample weights are post-stratified to match the noninstitutionalized U.S. population as represented by the U.S. Census Bureau (208). Stratification is the partitioning of a population of sampling units into exclusive categories (208). Stratification is used in NHANES for subpopulation analysis to increase the precision of estimates (208).

Research organizations, universities, healthcare providers and educators frequently use NHANES data to train, research, and educate. However, the primary users of NHANES data are the National Institutes of Health $(\mathrm{NIH})$, the Food and Drug Administration (FDA), the CDC and various other governmental agencies. These agencies rely on NHANES to assess the implementation and evaluation of various national programs and policies. Some of the most important accomplishments to date of NHANES have included national and global growth chart reference standards, blood lead level standards and policy to eliminate lead from gasoline and foodstuffs, national prevalence of overweight and diabetes and means of tracking national obesity and diabetes trends, national vitamin and mineral fortification regulations, identification of vulnerable populations for targeting national programs to reduce hypertension and cholesterol levels, and the development of new measures of lung function to help better describe the burden 
of asthma in the United States (209-215).

Volatile Organic Compounds in NHANES

The continuous NHANES survey has a changing focus on a variety of health and nutrition measurements to meet emerging needs. Through a partnership with the U.S. Environmental Protection Agency, NHANES has provided a platform for continued study of many important environmental influences on health. Three data release cycles, 2005/2006, 2011/2012, and 2013/2014 have included a urinary analysis of volatile organic compound metabolites in a subset of participants.

The metabolites were quantified using ultra performance liquid chromatography coupled with electro spray tandem mass spectrometry (UPLCESI-MS/MS) (25). Chromatographic separation is achieved in a column with 15 $\mathrm{mM}$ ammonium acetate and acetonitrile as the mobile phases. The eluent from the column is then ionized using an electrospray interface to generate and transmit negative ions into a mass spectrometer. Comparison of relative response factors (ratio of native analyte to stable isotope labeled internal standard) with known standard concentrations yield the individual analyte concentrations.

To the best of my knowledge only a handful of peer-reviewed publication have been published which utilized the NHANES urinary metabolites of VOCs (UM-VOCs) dataset. Four studies specifically address UM-VOC levels in children, one assesses differences in UM-VOCs in premium and discount cigarettes, and one analyzes UM-VOCs in marijuana users (216-220). Additionally, one study published in environmental toxicology and pharmacology in 2015 used the NHANES urinary metabolites of VOCs data to show that the distribution of 19 
urinary metabolites differs among some age, race, gender, and smoking demographic subsections (32). More specifically smokers were found to have significantly higher adjusted metabolites of acrolein, acrylamide, acrylonitrile, 1,3butadiene, carbon-disulfide, crotonaldehyde, cyanide, N,N-dimethylformamide, ethylbenzene-styrene, propylene oxide, styrene, and xylene as compared to nonsmokers (32). Female nonsmokers had lower adjusted levels of acrolein, carbondisulfide, and $\mathrm{N}, \mathrm{N}$-dimethylformamide metabolites than male nonsmokers but female smokers had higher levels of each of the metabolites than male smokers (32). Additionally, female smokers also had higher adjusted levels of 1,3butadiene, crotonaldehyde, cyanide, and ethylbenzene/styrene metabolites as compared to male smokers (32). Suggesting males and females metabolize and excrete some VOCs differentially. The study also reported that non-Hispanic whites had higher adjusted levels of 8 metabolites compared with non-Hispanic blacks and non-Hispanic blacks had significantly lower adjusted levels than Hispanics for 5 VOC metabolites and lower levels than non-Hispanic Asians for 6 metabolites (32). Hispanics had statistically higher levels than non-Hispanic Asians for 5 metabolites (32). Importantly the paper reported that levels of 11 metabolites increased with age (32).

Other methods of assessing VOC exposures have been employed in NHANES as well. Several studies have been published using serum VOC data. Cigarette smoke exposure has been established as a primary source of exposure for benzene, toluene, and styrene and an important source of exposure for xylene and ethylbenzene, showing clear dose-response relationships in NHANES data 
sets $(33,221)$. Marijuana use has also been established as a significant source of VOC exposure via serum VOC measurements from participants of the 2005-2012 NHANES surveys (220). NHANES serum VOC measurements have also been shown to have a positive dose-response effect with self-reported daily alcohol consumption (221).

A study assessing the effects of serum VOC measurements on neurobehavioral performance used principal component analysis to group 10 blood VOCs into 3 principle components mixtures, these three mixture principle components were used in quantile and ordinary least regression models. The study found impaired reaction times in the 90 th percentile of exposure (222). A study characterizing the national trends in VOCs from 1988-2004 utilized occupational and cotinine adjusted linear quantile models to show that while trends varied by VOC, there were overall decreasing tends for all quartiles of VOCs on the nation (223). For example, it was estimated that for $m, p$-xylene and tetrachloroethene there was a median exposure declined of 2.5 and 6.4 percent per year over the 15-year period, respectively (223). This pattern most probably reflects national occupational exposure regulations and general environmental regulations implemented during the time period (223).

Another method NHANES has used to assess VOC exposures is via passive air monitoring. In the 1999-2000 NHANES cohort, 851 participants wore passive exposure monitors for 48-72 hours, from which 10 VOCs were measured (207). A study using this data, published in 2014, found that overall, personal exposure was dominated by the home setting, although a large fraction of indoor 
VOC concentrations were due to outdoor sources (224). Another study looking at the 1999-2000 passive exposure monitoring dataset found that males had higher mean levels of all VOCs except toluene and benzene and that exposures were elevated in smokers and Hispanics (225). The study also found results which suggest that having an attached garage, closed windows, pumping gasoline and use of paint thinners results in higher exposure to VOCs (225). An analysis which built multiple linear regression models with the 1999-2000 passive exposure monitoring dataset to investigate the relationships between VOC levels in air and blood, while adjusting for effects of smoking and demographic factors, found that while levels of VOCs in blood were positively correlated with the corresponding air levels, the strength of association $\left(\mathrm{R}_{2}\right)$ widely varied $(0.02-0.68)(226)$.

A study assessing the associations between VOC exposures and asthma utilized factor analysis coupled with multiple logistic regression in the 1999-2000 passive exposure monitoring dataset. The study found the odds of physiciandiagnosed asthma was significantly higher among those exposed to aromatic compounds, adjusted $\mathrm{OR}=1.63(95 \% \mathrm{Cl}, 1.17-2.27)$, and a significant increase in odds was observed for one to two wheezing attacks for aromatic compounds, adjusted $\mathrm{OR}=1.68(95 \% \mathrm{Cl}, 1.08-2.61)$ and chlorinated hydrocarbons, adjusted $\mathrm{OR}=1.50(95 \% \mathrm{Cl}, 1.01-2.23)$ as compared to no wheezing (227). Another study which employed factor analysis and the 1999-2000 passive exposure monitoring dataset identified four common exposure sources: gasoline vapor and vehicle exhaust, methyl tert-butyl ether (MBTE) as a gasoline additive, tap water disinfectant products, and household cleaning products (228). 
NHANES VOC measurements have also been used to verify national mean levels of VOCs in various studies, to derive population-based blood/air distribution coefficients and to assess extreme values distributions $(30,229-232)$.

Study Population

All three waves of the National Health and Nutrition Examination Survey (NHANES) with data for urinary metabolites of volatile organic compounds (UMVOCs), 2005-2006, 2011-2012, and 2013-2014 were used in the analysis for the current study. Participants with available UM-VOC data were compiled/appended $\left(\mathrm{n}_{05 / 06}=3,516, \mathrm{n}_{11 / 12}=2,551, \mathrm{n}_{13 / 14}=2,724, \mathrm{~N}=8,791\right)$. This analysis was limited to individuals who were twenty years of age or older $(n=6030)$ and who have data available for all components of CMS ( $n=7070)$. Individuals who were pregnant $(n=233)$ or breastfeeding $(n=49)$ at the time of sample collection or who have diagnosed cardiovascular disease $(n=614)$ were excluded (Figure 2.1). 


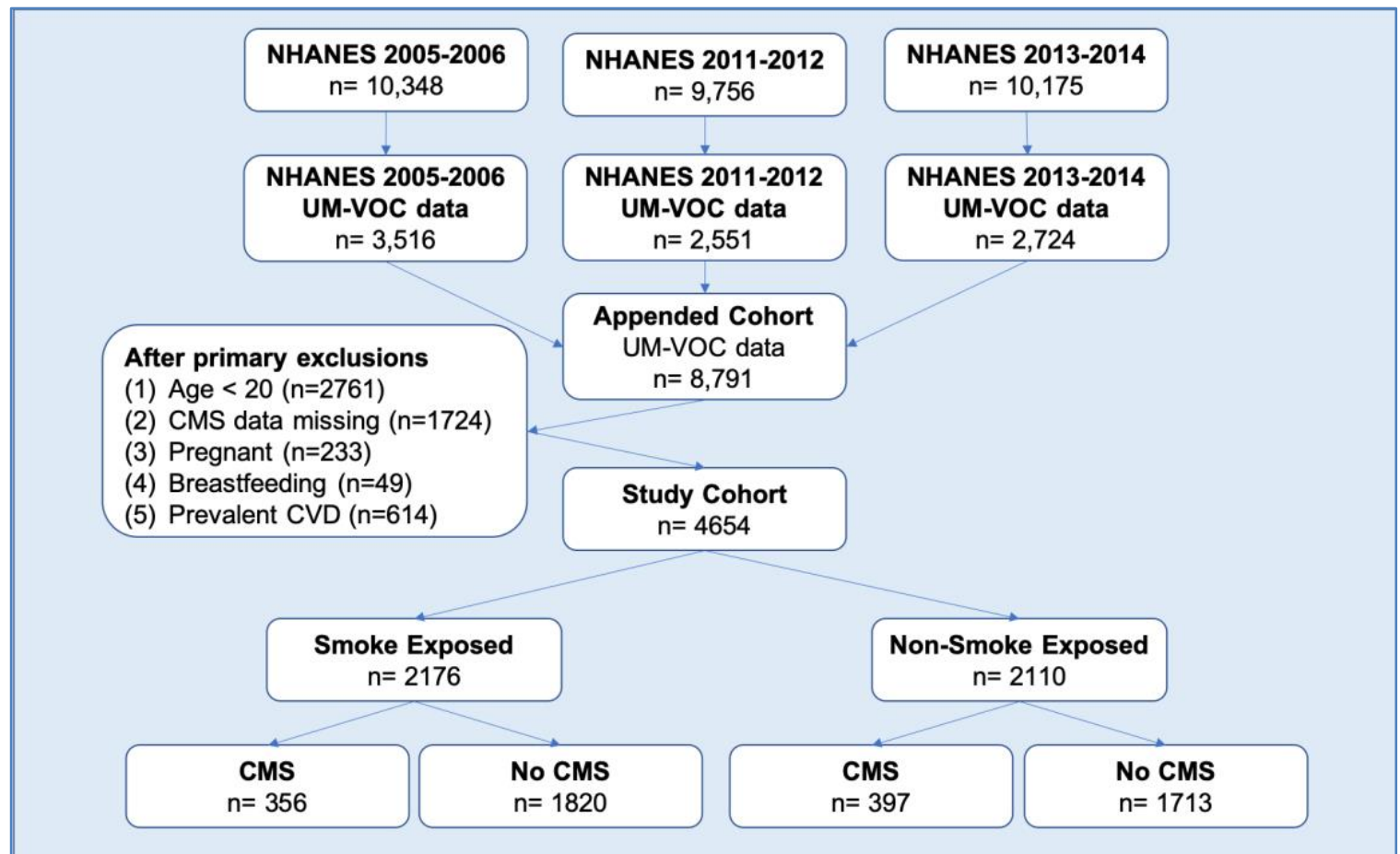

Figure 2.1. Schematic diagram depicting inclusion/exclusion criteria for 20052006, 2011-2012, and 2013-2014 NHANES subjects used to generate the study populations for further analysis.

To combine, or append the 2005-2006, 2011-2012, and 2013-2014 NHANES cohorts a new weighting variable was calculated. The 2005-2006 UMVOC cohort was from a $1 / 2$ sample of the full 2005-2006 NHANES cohort and the 2011-2012 and 2013-2014 UM-VOC cohorts were from a 1/3 sample of the full 2011-2012 and 2013-2014 NHANES cohorts. Because the sample size was smaller and therefore the weight of an observation may potentially be larger in the 2011-2012 and 2013-2014 cohorts, the weighting variable for the appended cohort was calculated by multiplying the $2005-2006$ weights by $3 / 7$, and the $2011-2012$ and 2013-2014 cohort weighting variables by $2 / 7$. The appended cohort weighting variable generated data representative of the non-institutionalized civilian population in the United States. 


\section{Exposure Measurements}

Concentrations of 27 urinary metabolites of volatile organic compounds (UM-VOCs) from 19 parent VOCs were measured by ultra performance liquid chromatography coupled with electro spray tandem mass spectrometry (UPLCESI/MSMS) (25). The Division of Laboratory Sciences (DLS), National Center for Environmental Health (NCEH), CDC, preformed the UM-VOC laboratory tests. Samples were stored at $-20^{\circ} \mathrm{C}$ until they were able to be shipped to NCEH for analysis. Metabolite levels lower than the detectable limit are reported in NHANES as the limit of detection divided by the square root of two (206). Exact laboratory protocol can be found in Alwis et al. (2012) and https://wwwn.cdc.gov/nchs/data/ nhanes/2011-2012/labmethods/UVOC_G_MET_VOC_Metabolites.pdf (25).

Residual stored urine samples were used to measure UM-VOCs from a one-half subsample of participants 12 years of age or older from the 2005-2006 NHANES wave. The 2011-2012 and 2013-2014 urine samples collected for UMVOC analysis were collected from a one-third subsample of participants 6 years of age of older (66). The UM-VOCs of interest and their parent VOC are listed in Table 2.1. 
Table 2.1. Parent Volatile Organic Compounds and Metabolites of Interest

\begin{tabular}{|c|c|c|}
\hline Parent VOC & $\begin{array}{l}\text { Common } \\
\text { Name }\end{array}$ & VOC metabolite (IUPAC name) \\
\hline \multirow{2}{*}{ Acrolein } & CEMA & N-Acetyl-S- (2-carboxyethyl)-L-cysteine \\
\hline & 3HPMA & N-Acetyl-S- (3-Hydroxypropyl)-L-cysteine \\
\hline \multirow[b]{2}{*}{ Acrylamide } & AAMA & \multirow{2}{*}{$\begin{array}{l}\text { N-Acetyl-S-(2-carbamoylethyl)-L-cysteine } \\
\text { N-Acetyl-S-(2-carbamoyl-2-hydroxyethyl)-L- } \\
\text { cysteine }\end{array}$} \\
\hline & GAMA & \\
\hline Acrylonitrile & CYMA & N-Acetyl-S-(2-cyanoethyl)-L-cysteine \\
\hline $\begin{array}{l}\text { Acrylonitrile, vinyl } \\
\text { chloride, ethylene } \\
\text { oxide }\end{array}$ & HEMA & N-Acetyl-S- (2-hydroxyethyl)-L-cysteine \\
\hline Benzene & PMA & N-Acetyl-S-(phenyl)-L-cysteine \\
\hline 1-Bromopropane & BPMA & N-Acetyl-S-(n-propyl)-L-cysteine \\
\hline \multirow{4}{*}{ 1,3-Butadiene } & DHBMA & N-Acetyl-S- (3,4-dihydroxybutyl)-L-cysteine \\
\hline & MHBMA1 & $\begin{array}{l}\text { N-Acetyl-S-(1-hydroxymethyl-2-propenyl)-L- } \\
\text { cysteine }\end{array}$ \\
\hline & MHBMA2 & \multirow{2}{*}{$\begin{array}{l}\text { N-Acetyl-S-(2-hydroxy-3-butenyl)-L-cysteine } \\
\text { N-Acetyl-S-(4-hydroxy-2-buten-1-yl)-L- } \\
\text { cysteine }\end{array}$} \\
\hline & MHBMA3 & \\
\hline Carbon-disulfide & TTCA & 2-Thioxothiazolidine-4-carboxylic acid \\
\hline Crotonaldehyde & HPMMA & $\begin{array}{l}\text { N-Acetyl-S-(3-hydroxypropyl-1-methyl)-L- } \\
\text { cysteine }\end{array}$ \\
\hline Cyanide & ATCA & 2-Aminothiazoline-4-carboxylic acid \\
\hline $\begin{array}{l}\mathrm{N}, \mathrm{N}- \\
\text { Dimethylformamide }\end{array}$ & AMCC & N-Acetyl-S-(N-methylcarbamoyl)-L-cysteine \\
\hline $\begin{array}{l}\text { Ethylbenzene, } \\
\text { styrene }\end{array}$ & PGA & Phenylglyoxylic acid \\
\hline Propylene oxide & 2HPMA & N-Acetyl-S-(2-hydroxypropyl)-L-cysteine \\
\hline \multirow[t]{2}{*}{ Styrene } & PHEMA & $\begin{array}{l}\text { N-Acetyl-S-(1-phenyl-2-hydroxyethyl-L- } \\
\text { cysteine + N-Acetyl-S-(2-phenyl-2- } \\
\text { hydroxyethyl)-L-cysteine }\end{array}$ \\
\hline & MA & Mandelic acid \\
\hline Tetrachloroethylene & TCVMA & N-Acetyl-S-(trichlorovinyl)-L-cysteine \\
\hline Toluene & BMA & N-Acetyl-S-(benzyl)-L-cysteine \\
\hline \multirow{2}{*}{ Trichloroethylene } & $1,2 \mathrm{DCVMA}$ & N-Acetyl-S-(1,2-dichlorovinyl)-L-cysteine \\
\hline & 2,2DCVMA & N-Acetyl-S-(2,2-dichlorovinyl)-L-cysteine \\
\hline \multirow{3}{*}{ Xylene } & DPMA & N-Acetyl-S-(dimethylphenyl)-L-cysteine \\
\hline & $2 \mathrm{MHA}$ & 2-Methylhippuric acid \\
\hline & $3 \& 4 \mathrm{MHA}$ & 3-Methylhippuric acid + 4-Methylhippur acid \\
\hline
\end{tabular}


Outcomes of interest

\section{Cardiometabolic Syndrome}

\section{Dichotomous health outcome}

A dichotomous variable generated to identify if an individual had prevalent cardiometabolic syndrome was created. The variable was generated using a modified World Health Organization (WHO) definition for clinically diagnosing cardiometabolic syndrome. The definition is considered modified because the definition for CMS used in this investigation does not include triglycerides; NHANES only conducts fasting analyses (needed for triglycerides measures) on a subset of participants which would have resulted in a significantly depressed sample size. For this analysis CMS was defined as the presence of dysglycemia, which is abnormal blood glucose levels (measured by self-reported type II diabetes mellitus (T2DM) status or $\mathrm{HbA} 1 \mathrm{c} \geq 5.7$ ), plus 2 or more of the following criteria:

1. Visceral obesity measured as body mass index (BMI) $\geq 30 \mathrm{~kg} / \mathrm{m} 2$ or a waist circumference $(\mathrm{WC})$ greater than $102 \mathrm{~cm}$ for men or greater than $88 \mathrm{~cm}$ for women.

2. Dyslipidemia classified by depressed HDL-cholesterol of $\leq 35 \mathrm{mg} / \mathrm{dL}$ in men or $\leq 39 \mathrm{mg} / \mathrm{dL}$ in women.

3. Hypertension defined as systolic blood pressure $\geq 140 \mathrm{~mm} \mathrm{Hg}$ or diastolic blood pressure $\geq 90 \mathrm{~mm} \mathrm{Hg}$ or self-report "currently taking blood pressure medication". 
4. Presence of Microalbuminuria measured as urinary albumin $\geq 30 \mathrm{mg} / \mathrm{g}$ (24).

Table 2.2 shows the definitions and variables for each risk factor of cardiometabolic syndrome that will be used in the subsequent investigation.

Table 2.2. Cardiometabolic Syndrome Risk Factor Variables and Definitions.

\begin{tabular}{|c|c|c|}
\hline Condition & Yes & No \\
\hline $\mathrm{CMS}^{*}$ & $\begin{array}{l}\text { Dysglycemia plus two other CM } \\
\text { risk factors }\end{array}$ & \\
\hline 1. Dysglycemia & Self-report T2DM or $\mathrm{HbA} 1 \mathrm{c} \geq 5.7$ & $\begin{array}{l}\text { No self-report } \\
\text { T2DM and } \\
\mathrm{HbA} 1 \mathrm{c}<5.7\end{array}$ \\
\hline \multicolumn{3}{|l|}{ 2. Visceral obesity } \\
\hline 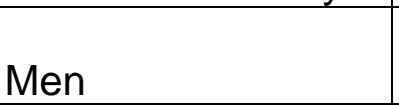 & $\begin{array}{l}>102 \mathrm{~cm} \text { waist circumference } \\
\text { and } / \mathrm{or} \mathrm{BMl}>30 \mathrm{~kg} / \mathrm{m} 2\end{array}$ & $\begin{array}{l}\leq 102 \mathrm{WC} \text { and } \\
\mathrm{BMI} \leq 30 \mathrm{~kg} / \mathrm{m} 2\end{array}$ \\
\hline Women & $\begin{array}{l}>88 \mathrm{~cm} \text { waist circumference } \\
\text { and } / \mathrm{or} \mathrm{BMl}>30 \mathrm{~kg} / \mathrm{m} 2\end{array}$ & $\begin{array}{l}\leq 88 \mathrm{WC} \text { and } \\
\mathrm{BMI} \leq 30 \mathrm{~kg} / \mathrm{m} 2\end{array}$ \\
\hline †Triglycerides & $\geq 1.7 \mathrm{mmol} / \mathrm{L}$ & $<1.7 \mathrm{mmol} / \mathrm{L}$ \\
\hline \multicolumn{3}{|l|}{ 3. HDL-cholesterol } \\
\hline Men & $\mathrm{HDL} \leq 35 \mathrm{mg} / \mathrm{dL}$ & $>35 \mathrm{mg} / \mathrm{dL}$ \\
\hline Women & $\mathrm{HDL} \leq 39 \mathrm{mg} / \mathrm{dL}$ & $>39 \mathrm{mg} / \mathrm{dL}$ \\
\hline 4. Hypertension & $\begin{array}{l}\text { blood pressure medication or } \\
\text { Systolic BP } \geq 140 \mathrm{~mm} \mathrm{Hg} \text { or } \\
\text { Diastolic BP } \geq 90 \mathrm{~mm} \mathrm{Hg}\end{array}$ & $\begin{array}{l}\text { No medication } \\
\text { and } \\
<140 / 90 \mathrm{~mm} \mathrm{Hg}\end{array}$ \\
\hline 5. Microalbuminuria & $\begin{array}{l}\text { urinary albumin:creatinine ratio } \\
\geq 30 \mathrm{mg} / \mathrm{g}\end{array}$ & $<30 \mathrm{mg} / \mathrm{g}$ \\
\hline \multicolumn{3}{|c|}{$\begin{array}{l}\text { CMS, Cardiometabolic Syndrome; CM, Cardiometabolic; T2DM, type } 2 \\
\text { diabetes mellitus; BP, blood pressure }\end{array}$} \\
\hline \multicolumn{3}{|c|}{$\begin{array}{l}\text { *T2DM or HbA1c } \geq 5.7 \text { plus } 2 \text { or more } \mathrm{CM} \text { risk factor criteria } \\
\text { †Triglycerides were removed as a risk factor for this analysis because } \\
\text { only a fasting subset of participants have triglyceride measurements. }\end{array}$} \\
\hline
\end{tabular}

\section{Ordinal health outcome}

An ordinal score variable was generated to assess an individual's severity of cardiometabolic risk factors. The variable was generated using the above modified World Health Organization (WHO) definitions and variables for clinically diagnosing cardiometabolic syndrome. For the ordinal CMS-Risk Score (CMS-RS) 
outcome variable individuals were assigned a score based on individual prevalence of CMS risk factors. Individuals with 0 risk factors were assigned a CMS-RS of 0 . Individuals with 1 risk factor were assigned a CMS-RS of 1 . Individuals with 2 risk factors were assigned a CMS-RS of 2. Individuals with 3 risk factors, but not considered to have CMS (not having dysglycemia as a risk factor) were assigned a CMS-RS of 3 . Individuals with 4 risk factors, but not considered to have CMS (not having dysglycemia as a risk factor) were assigned a CMS-RS of 4 . Individuals with CMS, that is they had dysglycemia plus 2 additional risk factors were assigned a CMS-RS of 5. Individuals with CMS plus 1 additional risk factor, that is they had dysglycemia plus 3 additional risk factors were assigned a CMS-RS of 6 . Finally, individuals with CMS plus 2 additional risk factors, that is they had dysglycemia plus 4 additional risk factors were assigned a CMS-RS of 7 . Table 2.3 shows the number of risk factors and distinction of diagnosable CMS for each CMS-RS.

Table 2.3. Cardiometabolic Syndrome Risk Score.

\begin{tabular}{|c|c|c|}
\hline CMS-RS & Number of Risk Factors & CMS \\
\hline 0 & 0 & No \\
1 & 1 & No \\
2 & 2 & No \\
3 & 3 & No \\
4 & 4 & No \\
5 & $\mathrm{CMS}$ & Yes \\
6 & $\mathrm{CMS}+1$ & Yes \\
7 & $\mathrm{CMS}+2$ & Yes \\
\hline
\end{tabular}

Note: $\mathrm{CMS}-\mathrm{RS}=$ cardiometabolic syndrome risk score; $\mathrm{CMS}=$ cardiometabolic syndrome

Covariates of Interest

Cardiometabolic syndrome is thought to develop as a result of both genetic 
factors and acquired/environmental factors. It is hypothesized that genetic factors predispose individuals to magnified effects of environmental exposures, which in conjunction amplify the effects of cardiovascular and metabolic adversities (199). Of the immense number of potential covariates that could be considered, this analysis will a priori consider socioeconomic status, gender, age, ethnicity, alcohol consumption patterns, physical activity, body mass index $(198,200,201)$. Other covariates may additionally be considered for the analysis.

\section{Sociodemographic}

Participants self-reported information regarding age, educational statues and race. Age information was specified as continuous. Race information was classified as either Non-Hispanic White, Non-Hispanic Black, Mexican American Other Hispanic, and Other/Multi-Racial. Educational status was classified according to traditional U.S. grades; Less than high school graduate/GED, versus more than a high school graduate/GED was used in this analysis. Annual house hold income was used to assess Socio-economic status. Having an annual house hold income of less than $\$ 20,000$ a year was defined as the cutoff point for living below the poverty level (above would indicate not).

\section{Anthropometry}

Body mass index (BMI) was calculated as weight in Kilograms divided by the square of height in meters. BMI was assessed as a continuous variable. No modifications or adjustments were made for race or ethnic group. Within the CMS definition individuals were considered to be obese with a $\mathrm{BMI}>30 \mathrm{~kg} / \mathrm{m} 2$. Waist circumference was measured to the nearest $0.1 \mathrm{~cm}$ at the level of the iliac crest. 
Females were considered obese within the CMS definition if they had a waist circumference of $>88 \mathrm{~cm}$. Males were considered obese in the CMS definition if they had a waist circumference of $>102 \mathrm{~cm}$.

\section{Medical History}

Participants participated in a home interview. They were asked about family members medical history. For the purpose of this question, a family member was defined as an immediate blood relation living or deceased, which would include grandparents, parents, brothers and sisters. Participants were asked about family history and personal history of doctor diagnosed heart disease and cancer.

Blood pressure was measured using a mercury sphygmomanometer. These measurements were taken while the participant was seated. Participants were asked about, physician diagnosed hypertension, or current medication use for elevated blood pressure.

Non-fasting serum levels of HDL-cholesterol were measured using the Roche/Boehringer-Mannheim Diagnostics direct HDL method.

In the NHANES III data sets, fasting measures of triglycerides were obtained for only approximately $50 \%$ of participants attending the morning examinations. This resulted in a significantly reduced sample size of participants with triglyceride measurements making a measure of triglycerides unavailable for use in the definition for CMS for the analysis.

\section{Lifestyle Factors}

Self-reported tobacco use and self-reported use of tobacco and cigarette use questions and a question about the occurrence of in-home smoking were used 
in conjunction with serum cotinine levels to classify each participant as a 'current smoker", "secondhand smoke exposed", or "non-exposed" to smoke. A variable "Smoking exposure" combined these self-reported responses with serum cotinine cut points of current smoker ( $>15 \mathrm{ng} / \mathrm{mL}$ cotinine), secondhand smoke exposed ( $>0.05 \mathrm{ng} / \mathrm{mL}$ cotinine to $<15 \mathrm{ng} / \mathrm{mL}$ coninine), non-exposed $(<0.05 \mathrm{ng} / \mathrm{mL}$ coninine).

With respect to physical activity, the participants were asked about work, and recreational activity. If a participant reported no vigorous or moderate work or recreational physical activity they were considered physically inactive. Activity was defined by self-reported heart rate or breathing increase; activities that require hard physical effort and cause large increases in breathing or heart rate, and 'moderateintensity activities' are activities that require moderate physical effort and cause small increases in breathing or heart rate for more than 10 minutes.

\section{Power}

A minimum detectable effect size for each UM-VOC was calculated. To test the hypothesis $H_{0}: \mu_{1}=\mu_{2}$ vs. $H_{1}: \mu_{1} \neq \mu_{2}$ with the alterative $\left|\mu_{1}-\mu_{2}\right|=\Delta$, significance level $\alpha=0.05$, and power $80 \%$ a two-tailed minimum detectable effect size was calculated using the equations Power $=\Phi\left[-z_{1-\alpha / 2}+\left(\frac{\Delta}{\sqrt{\sigma_{1}^{2} / n_{1}+\sigma_{2}^{2} / n_{2}}}\right)\right]$.

Where $\left(\mu_{1}, \sigma_{1}\right)$ and $\left(\mu_{2}, \sigma_{2}\right)$ are the means and standard deviation of each nonsurvey weighted creatinine normalized log-transformed UM-VOC for participants with CMS and participants without CMS and $\mathrm{n}_{1}$ and $\mathrm{n}_{2}$ and the sample sizes of the two respective groups (Table 2.4). 
For a two-tailed difference in means there was sufficient power to detect differences in at least 8 non-survey weighted, creatinine normalized, logtransformed UM-VOCs.

Table 2.4. Non-Survey Weighted Minimal Detectable Difference Calculation Data

\begin{tabular}{|c|c|c|c|c|c|c|c|c|c|c|c|}
\hline \multirow[b]{2}{*}{ Parent Compound } & \multirow[b]{2}{*}{ Metabolite } & \multicolumn{3}{|c|}{ CMS } & \multicolumn{3}{|c|}{ No CMS } & \multicolumn{4}{|c|}{ Minimal } \\
\hline & & $\mathrm{n}$ & mean & SD & $\mathrm{n}$ & mean & SD & $\begin{array}{l}\text { Difference } \\
\text { in means }\end{array}$ & $\begin{array}{l}\text { Detectable } \\
\text { Difference }\end{array}$ & Z score & Power \\
\hline \multirow[t]{2}{*}{ Acrolein } & CEMA & 791 & 4.741 & 0.781 & 3769 & 4.540 & 0.825 & $0.201^{*}$ & 0.088 & 0.901 & 0.816 \\
\hline & 3HPMA & 791 & 5.570 & 0.937 & 3765 & 5.568 & 0.977 & 0.002 & 0.104 & 0.851 & 0.803 \\
\hline \multirow[t]{2}{*}{ Acrylamide } & AAMA & 781 & 3.889 & 0.757 & 3723 & 3.995 & 0.818 & $0.105^{*}$ & 0.087 & 0.906 & 0.817 \\
\hline & GAMA & 791 & 2.477 & 0.677 & 3782 & 2.494 & 0.697 & 0.017 & 0.078 & 0.954 & 0.830 \\
\hline Acrylonitrile & CYMA & 790 & 1.293 & 1.891 & 3751 & 1.495 & 2.051 & $0.202^{*}$ & 0.200 & 0.699 & 0.758 \\
\hline $\begin{array}{l}\text { Acrylonitrile, vinyl chloride, } \\
\text { ethylene oxide }\end{array}$ & HEMA & 791 & -0.046 & 0.893 & 3762 & 0.037 & 0.964 & 0.083 & 0.100 & 0.855 & 0.804 \\
\hline Benzene & PMA & 783 & -0.459 & 0.748 & 3748 & -0.476 & 0.775 & 0.017 & 0.085 & 0.919 & 0.821 \\
\hline 1-Bromopropane & BPMA & 781 & 1.462 & 1.335 & 3752 & 1.495 & 1.368 & 0.033 & 0.144 & 0.776 & 0.781 \\
\hline \multirow[t]{2}{*}{ 1,3-Butadiene } & DHBMA & 793 & 5.687 & 0.493 & 3781 & 5.587 & 0.526 & $0.100 *$ & 0.059 & 1.079 & 0.860 \\
\hline & MHBMA3 & 779 & 1.901 & 0.984 & 3721 & 1.881 & 1.076 & 0.020 & 0.110 & 0.825 & 0.795 \\
\hline Carbon-disulfide & TTCA & 793 & 2.774 & 1.090 & 3782 & 2.691 & 1.035 & 0.083 & 0.118 & 0.838 & 0.799 \\
\hline Crotonaldehyde & HPMMA & 791 & 6.248 & 0.930 & 3751 & 6.138 & 0.981 & $0.109 *$ & 0.103 & 0.850 & 0.802 \\
\hline Cyanide & ATCA & 780 & 4.642 & 1.159 & 3728 & 4.507 & 1.227 & $0.136^{*}$ & 0.127 & 0.795 & 0.787 \\
\hline$N, N$ - Dimethylformamide & AMCC & 791 & 5.072 & 0.860 & 3779 & 5.000 & 0.961 & 0.072 & 0.097 & 0.857 & 0.804 \\
\hline Ethylbenzene, styrene & PGA & 789 & 5.124 & 0.844 & 3747 & 4.963 & 0.987 & $0.162 *$ & 0.096 & 0.849 & 0.802 \\
\hline Propylene oxide & 2HPMA & 788 & 3.509 & 0.933 & 3763 & 3.555 & 0.979 & 0.046 & 0.104 & 0.851 & 0.803 \\
\hline \multirow[t]{2}{*}{ Styrene } & PHEMA & 786 & -0.325 & 0.828 & 3752 & -0.330 & 0.857 & 0.005 & 0.093 & 0.887 & 0.813 \\
\hline & MA & 783 & 4.928 & 0.732 & 3739 & 4.920 & 0.710 & 0.009 & 0.083 & 0.938 & 0.826 \\
\hline Toluene & BMA & 792 & 2.050 & 0.890 & 3775 & 1.974 & 0.880 & 0.076 & 0.098 & 0.877 & 0.810 \\
\hline \multirow[t]{2}{*}{ Xylene } & $2 \mathrm{MHA}$ & 766 & 3.432 & 1.162 & 3655 & 3.571 & 1.099 & $0.139 *$ & 0.127 & 0.825 & 0.795 \\
\hline & $3 \& 4 \mathrm{MHA}$ & 791 & 5.414 & 1.090 & 3776 & 5.502 & 1.079 & 0.088 & 0.119 & 0.827 & 0.796 \\
\hline
\end{tabular}

*Difference in means sufficient to detect minimum detectable difference. tmean and standard deviation for non-survey-weighted creatinine normalized log-transformed UM-VOCs

\section{Statistical Analysis Overview}

All statistical analyses were performed using SAS, version 9.4 or R 3.3.2. SAS survey procedures were used in all appropriate analyses and sampling design variables, sample weights, cluster and stratification weightings were incorporated in all appropriate analyses to address the complex survey design of NHANES. SAS survey procedures incorporate the complex sampling design and sampling weights, which without accounting for the cluster design and differential probability of selection in NHANES would result in bias parameter estimates and overstated statistical significance. Specific analyses are discussed in subsequent sections. 
The majority of the current knowledge, associations, and regulations of VOCs and cardiovascular and metabolic dysfunction have been linked to or studied in terms of particulate matter (PM) exposure. However, more than $98 \%$ of urban air pollution is composed of gasses or vapor-phase compounds such as $\mathrm{CO}$, nonmethane hydrocarbons, and volatile organic compounds (VOCs). Each of these components of ambient air pollution may have independent, synergistic, or antagonistic effects with each other and/or with PM; for this reason the effects of VOCs on health need to be considered both in an individual manner (addressed in aim 2 and aim 3) and also from a global viewpoint i.e. multipollutant combinatory modeling, (addressed in aim 4) (14).

The hypothesis is that CMS is positively associated with increasing UMVOCs. The methods for exploring this hypothesis used the 2005-2006, 2011-2012, and 2013-2014 NHANES UM-VOC sub-sample cohorts, as described above. The hypothesis was tested as outlined in the following aims.

Analyses of Aim 1

Specific aim 1 is to statistically describe and compare distributions, trends, and cohort characteristics of the 2005-2006, 2011-2012, and 2013-2014 NHANES UM-VOC sub-sample.

Demographic characteristics of the cohort including sample size, mean, percent, and standard error were generated using SAS survey procedures SURVEYFREQ and SURVEYMEANS. Demographic characteristic proportional distribution statistics describe and compare the cohort according to CMS status. The two mutually exclusive groups were individuals with cardiometabolic 
syndrome and individuals without cardiometabolic syndrome. SAS versions 9.4 SURVEYREG was used to generate weighted basic linear regression models and calculate $p$-values for the differences in continuous demographic variables by CMS status and NHANES wave. SAS SURVEYFREQ was used to conduct first-order Rao-Scott chi-square tests to generate $p$-values across levels of categorical variables. These survey procedures were also used to generate demographic descriptive and comparative statistics between NHANES waves (2005/06, 2011/12, and 2013/14).

The Rao-Scott chi-square test is a design-adjusted version of the Pearson chi-square test, which corrects for design effects of the table cell proportion estimates and, for two-way tables, the design effects of the marginal proportion estimates (234). The Rao-Scott design-adjusted chi-square goodness-of-fit test involves differences in observed and expected frequencies with a default null hypothesis of equal proportions. For this analysis the first-order Rao-Scott chisquare statistic for the goodness-of-fit test was computed as $Q_{R S I}=Q_{P} / D$. Where $Q_{P}$ is the Pearson chi-square based on estimated totals and $D$ is the first-order design correction $D=\sum_{c}\left(1-\widehat{P}_{c}\right) \operatorname{Deff}\left(\widehat{P}_{c} /(c-1)\right.$. Where $\operatorname{Deff}\left(\widehat{P}_{c}\right)=\widehat{\operatorname{Var}}\left(\widehat{P}_{c}\right) /$ $\operatorname{Var}_{s r s}\left(\hat{P}_{c}\right)$. Where $\hat{P}_{c}$ equals the proportion estimate for level $c$ and $\widehat{\operatorname{Var}}\left(\hat{P}_{c}\right)$ is the variance estimate. For a first-order one-way table with $C$ levels, the Pearson chisquare is computed as $Q_{P}=\left(\frac{n}{\hat{N}}\right) \sum_{c}\left(\widehat{N_{c}}-{\widehat{E_{c}}}^{2} / E_{c}\right.$. Where $\mathrm{n}$ is the sample size, $\widehat{N}$ is the estimated overall total, $\widehat{N}_{c}$ is the estimated total for level $c$ and $E_{c}$ is the expected total level of $c$ under the null hypothesis of equal proportions. The expected total for each level is $E_{c}=\widehat{N} / C$. Under the null hypothesis the first-order 
Rao-Scott chi-square statistic $Q_{R S I}$ approximately follows the chi-square distribution with $(C-1)$ degrees of freedom (234). First-order Rao-Scott chisquare tests for two-way tables tests associations between row and column variables; for two-way tables $Q_{P}=\left(\frac{n}{\widehat{N}}\right) \sum_{r} \sum_{c}\left(\widehat{N_{r c}}-\widehat{E_{r c}}\right)^{2} / E_{r c}$, where $\mathrm{n}$ is the sample size, $\widehat{N}$ is the estimated overall total; $\widehat{N_{r c}}$ is the estimated total for cell $(r, c)$ and $E_{r c}$ is the expected total cell $(r, c)$ under the null hypothesis of equal proportions/no association. The expected total for each level is $E_{r c}=\widehat{N}_{r} \widehat{N}_{c} / \widehat{N}$ (234). Under the null hypothesis the first-order Rao-Scott chi-square statistic $Q_{R S I}$ approximately follows the chi-square distribution with $(R-1)(C-1)$ degrees of freedom (234).

Urinary metabolites of volatile organic compounds in the NHANES dataset were first assessed for level of detection insufficiencies. The level of detection or LOD is the lowest quantifiable level that is able to be detected by the laboratory protocol for each metabolite (235). For this analysis, any metabolite with less than $15 \%$ of participants at or below the LOD was removed from any further analysis due to unreliability of statistical tests with very limited sample sizes. For metabolites with greater than $15 \%$ of samples measured above LOD; values of analytes with analytic results below the LOD, were reported by NHANES using the standard imputations method of $L O D / \sqrt{2}$ (236). UM-VOCs were normalized to individual level urinary creatinine concentration to adjust for dilution/concentration of the urine.

The same SAS survey procedures as discussed above were used to describe the basic descriptive statistics for volatile organic compound urinary 
metabolites. The geometric mean, median, standard error, and quartiles distributions for each metabolite by NHANES wave (2005-06, 2011-12, and 201314) as well as for the full-appended cohort were described. The appended cohort was further stratified by CMS status and the same descriptive statistics were used to describe UM-VOCs by CMS status; UM-VOCs by CMS status were also be further stratified by smoking status. Additionally, levelplots using the lattice package in the statistical software $R$ version 3.3.2 were used to generate correlation matrices in order to gain an initial assessment of any possible collinearity of the UM-VOCs.

Analyses of Aim 2

Specific aim 2 is to study the effects of individual continuous UM-VOCs and prevalent CMS after adjusting for select covariates.

The main objective for this aim was to determine whether particular UMVOCs, treated continuously, are associated with CMS status, measured as a dichotomous variable. The null hypothesis was that a particular UM-VOC was not associated with CMS (Ho: B1=0), with an alternative hypothesis being that a particular UM-VOC is associated with CMS $(\mathrm{H} 1$ : B1 $\neq 0)$.

Multivariable logistic regression models were used to determine whether a particular UM-VOC, treated as a continuous variable, was associated with CMS prevalence. Variables were considered for inclusion in the final model based on both a priori knowledge of an important role of a variable in the relationship between UM-VOCs and CMS, as well as their statistical significance in the model (discussed in more detail below). 
A priori adjustments were identified as important from both the scientific literature as well as from statistically significant differences in population descriptive statistics stratified by CMS status, identified in the aim one of this dissertation. Age was significantly different ( $p$-value $<0.0001)$ between individuals with and without CMS in the study cohort. Individuals with CMS were more likely to be older than individuals without CMS. Older age is associated with the majority of the diseases that define CMS and the prevalence of CMS significantly increases with age (192). Significant increases in the prevalence of CMS are observed at about age 30 , with peak increases in prevalence for men occurring between the ages of 50 to 70 ; and peak increases in prevalence for women occurring approximately a decade later, between the ages of 60 to 80 (198). Sex was not significantly different between individuals with and without CMS in the study cohort but significant sex differences exist for the majority of the metabolic and cardiovascular conditions used the define CMS $(3,198)$. Obesity, systolic blood pressure, BMI, and diabetes have all been shown to vary significantly by sex (237). Additionally, rates of change in the prevalence of the CMS risk factors have been reported to be different by sex since the 1980 s, indicating the need to account for these differences in population-level analyses(237). Race was significantly different ( $p$-value $<0.0001$ ) between individuals with and without CMS in the study cohort. Rates of cardiometabolic syndrome and a number of the conditions used to define CMS are highly stratified by ethnicity. Mexican American women have the highest rates of CMS in America while Black men have the lowest rates (202). These ethnic differences persist even after adjusting for age, smoking and drinking 
habits, socioeconomic status, physical inactivity, and menopausal status among women $(198,238,239)$. Twelve of the $21 \mathrm{VOC}$ concentrations in this investigation were significantly different between the NHANES cohort years (2005/06, 2011/12, and 2013/14) (Table A.3). Konkle et. al confirms these observations in a 2020 publication showing significant increasing trends in urinary VOC metabolite concentrations over this time period (2005 to 2014) (10).

Models were initially a priori adjusted for age, sex, race, and NHANES cohort year. Interactions between each of these terms were assessed using product terms in the model; a significant interaction was observed with sex and race. Additional potential variables of interest that may have had some impact on the relationship, such as cancer and socioeconomic status, were assessed based on a liberal $p$-value threshold for statistical significance $(p<0.2)$. These analyses did not reveal any further significant effects and did not confound the association between VOCs and CMS, and therefore were not included in the final models. Covariates were removed individually via backwards elimination to assess potential confounding effects with the remaining variables in the model. Variables that resulted in a $10 \%$ change of the odds ratio (OR) of the exposure of interest would be re-inserted into the model. Based on the significance of the covariates and interaction terms $(p<0.1)$, and the lack of confounding, the final models were adjusted for age, sex and race interaction, and NHANES cohort year.

Age, sex, race, and NHANES cohort year as well as the interaction between race and sex were found to be significant in multivariate logistic regression models of individual VOCs and CMS. In the interest of parsimony and comparability of 
models, all adjusted models were adjusted for age, sex, race, sex and race interaction, and NHANES cohort year.

Because of the large number of exposures examined, a multiple comparisons adjustment was implemented by using a new significance threshold identified by a modified Bonferroni correction. The Bonferroni adjustment is one of the most frequently and widely used multiple testing approaches because of its ease of uses and intuitive interpretability (240). However, the traditional Bonferroni approach is often viewed as being overly conservative and suffers from poor statistical performance when used with correlated test data. Therefore, adaptation of the traditional Bonferroni adjustments have been developed to help overcome these shortcomings (241). One such modified Bonferroni adjustment is based on the concept of intraclass correlation to imposes a correction for the number of classes/groups of correlated data, rather than adjusting for every test; therefore, producing a less conservative adjustment (242).

For this analysis the number of classes of correlated data were identified by Factor Analysis. The corrected significance threshold was calculated by dividing the type I error rate, $\alpha(0.05)$ by the number of independent classes/groupings of VOC metabolites identified by factor analysis. The factor analysis was performed using log-transformed metabolites.

Due to the number of moderate to highly correlated data in the tobacco smoke exposed cohort (Figure A.1, and Figure A.2), the factor analysis was conducted using only the non-tobacco smoke exposed sub-populations of the study cohort. Additionally, UM-VOCs with less than $40 \%$ of sample above LOD 
were excluded in the factor analysis; therefore, $16 \mathrm{UM}$-VOCs were included in the factor analysis. Creatinine normalized log-transformed UM-VOCs were assessed. The maximum likelihood estimation factor extraction method with squared multiple correlations prior communality estimates were used in the SAS Proc FACTOR procedure.

Analyses of Aim 3

Specific aim 3 is to evaluate the dose-response relationships of individual categorical UM-VOC exposures and CMS.

The objective of this aim was to determine whether a dose-response relationship exists between each UM-VOC and CMS. The dose-response relationship was explored using urinary metabolite concentrations as an estimation of dose. Because tobacco smoke exposure was used as a chronic high-dose exposure and the dose-response analyses were not impacted but correlations; the analysis in this section was conducted using the entire study cohort. To visually assess if a dose-response relationship exists, each UM-VOC was placed into equally numbered quintiles i.e. approximately the same number of participants were placed in each quintile, and figures were generated by plotting CMS prevalence on the $y$-axis against UM-VOC quantile concentration cut-points on the $\mathrm{x}$-axis. To examine trends that were not adjusted for any covariables, chi-square tests for trend were applied to UM-VOC quantile concentration cut-points. Logistic regression models were built to assess trends after adjusting for age, sex, race, and NHANES cohort year. In the models, the UM-VOC quintiles were first treated ordinally to assess linear trends, and second treated categorically, to examine 
whether any of the quintiles had significantly higher odds of CMS versus the referent category (lowest exposure quintile).

Analysis of Aim 4

Specific aim 4 is to explore combinatory effects of VOCs on CMS.

The main research question for this aim was: which combinatorial UMVOCs are more likely to predict CMS? To examine this research question, exploratory analyses were conducted, including: ordinal logistic regression using a CMS-risk score (CMS-RS); a traditional backwards-selection regression modelbuilding approach to examine two-way interactions; factor analysis; and LASSO penalized regression; as well as a multipollutant approach using a cumulative VOC exposure score. These methods used in this aim are complementary of each other and are used to identify potential important groupings and interactions among UMVOCs, and their associations with CMS. The methods used in this aim were independent, unrelated exploratory analyses conducted in order to add evidence that combinatory VOC exposures are predictive of CMS.

\section{Ordinal Logistic Regression}

The ordinal logistic regression analysis was conducted assuming that an intrinsic order in the increase in CMS components is associated with an increased disease state, but that the distance between the level of increase in disease progression is unknown. Due to the non-interval nature of the CMS-RS (OLS regression would be used if CMS-RS were interval data) and the desire to retain the information contained in the order of the CMS-RS, ordinal logistic regression was chosen for this analysis. 
To produce the ordinal CMS-RS, each of the components of the CMS definition (abnormal blood glucose levels, visceral obesity, dyslipidemia, low HDLcholesterol, hypertension, and the presence of microalbuminuria) were dichotomized as present or absent. Individuals received a score reflective of the number of components of CMS they had at the time of data collection (Table 2.3). These scores were assessed in an ordinal manner for associations with UM-VOCs.

Table 2.3. Cardiometabolic Syndrome Risk Score.
\begin{tabular}{|c|c|c|}
\hline CMS-RS & Number of Risk Factors & CMS \\
\hline 0 & 0 & No \\
1 & 1 & No \\
2 & 2 & No \\
3 & 3 & No \\
4 & 4 & No \\
5 & $\mathrm{CMS}$ & Yes \\
6 & $\mathrm{CMS}+1$ & Yes \\
7 & $\mathrm{CMS}+2$ & Yes \\
\hline
\end{tabular}

Note: $\mathrm{CMS}-\mathrm{RS}=$ cardiometabolic syndrome risk score; $\mathrm{CMS}=$ cardiometabolic syndrome

In contrast to binary logistic regression, which models the response itself, ordinal logistic regression uses logistic regression to model the probability of events. In ordinal logistic regression a cumulative logit model assumes that the effect of an explanatory variable is identical for all modeled logits. This assumption is known as the assumption of proportional odds (243). Survey-weighted proportional-odds cumulative logit models were used to investigate the response, CMS-RS with respect to each individual UM-VOC. The Chi-Square Score test was used to test the proportional odds assumption. For simplicity a model with one predictor would take the form: $\operatorname{logit} P(Y \leq j)=\alpha_{j} \beta x$ and the cumulative probabilities would be $P(Y \leq j)=\exp \left(\alpha_{j}+\beta_{x}\right) /\left(1+\exp \left(\alpha_{j}+\beta_{x}\right)\right)$. Beta is 
constant; therefore, the curves of the cumulative probabilities plotted against $x$ are parallel. The odds ratios for these models are proportional to the differences between $\mathrm{x}_{1}$ and $\mathrm{x}_{2}$ where beta is the constant of proportionality: $\exp \left[\beta\left(x_{1}-x_{2}\right)\right]$ (233).

\section{Backwards Selection}

Potential two-way interactions between UM-VOCs were identified using a binary logistic regression backward selection method. The backwards elimination technique begins with a full model, including all effects. The effects with the smallest contribution were removed one by one until a specified threshold was met; for this analysis effects were removed from the model until all effects were significant at the 0.05 level. This analysis was conducted using CMS (dichotomous), rather than the ordinal CMS-RS because as the number of predictors increases in an ordinal logistic regression model the less likely that the proportional odds assumption will be met. Initially, an unadjusted logistic regression model was used to identify the interactions of interest. Subsequently, the identified important interactions were placed in an adjusted survey weighted logistic regression model.

Log-transformed metabolites with greater that $40 \%$ of the samples above the LOD were used to assess two-way interactions. To minimize any potential multicollinearity, 2-way interactions were assessed in non-tobacco smoke exposed individuals, and only 1 metabolite per parent VOC was selected for inclusion in the logistic regression backwards selection analysis. A representative metabolite for

each parent VOC was selected. If a parent VOC had multiple metabolites, the 
metabolite with the greatest significance was selected; if none of the metabolites were significant, the most abundant metabolite was selected to represent the parent VOC.

To assess if the response of CMS depends on the interaction identified from the backward selection model, significant interaction terms were then placed $\left(\mathrm{CMS}=\beta_{0}+\beta_{1} \mathrm{UM}-\mathrm{VOC}_{1}+\beta_{2} \mathrm{UM}-\mathrm{VOC}_{2}+\beta_{3}\left(\mathrm{UM}-\mathrm{VOC}_{1}\right.\right.$ * UM-VOC $)+$ adjustments $)$ into survey weighted ordinal logistic regression models adjusted for age, sex, race, the interaction between sex and race, and NHANES cohort year. If the interaction term was not significant ( $p$-value $<0.05$ ) in the adjusted survey weighted logistic regression model, then a model removing the interaction term while maintaining the individual UM-VOCs that made up the interaction term was built and assessed, if the UM-VOCs were significant ( $p$-value $<0.05)\left(C M S=\beta_{0}+\beta_{1} U_{M}-V_{O C}+\beta_{2} U M-\right.$ $\mathrm{VOC}_{2}+$ adjustments)

\section{Factor Analysis}

Another method that was used to examine the effects of multiple UM-VOCs was factor analysis. Factor analysis is a statistical method that describes the variability among variables and is often used as a dimension reduction technique. Factor analysis groups correlated data into unobserved terms known as factors. The purpose of the factor analysis in this investigation was to group the UM-VOCs into a reduced number of unobserved latent variables or factors.

After the initial extraction of the 3 latent factors, preformed in aim 2, a rotated factor pattern analysis in the non-tobacco smoke exposed cohort was conducted using UM-VOCs with greater the $40 \%$ above LOD (16 metabolites). The maximum 
likelihood estimation factor extraction method with squared multiple correlations prior communality estimates were used in the SAS Proc FACTOR procedure. An oblique rotation, which allows the factors to be correlated with one another, was implemented via the quartimin rotation. A path diagram showing directed links from the 3 factors identified in aim 2 to variables that are associated with a 0.3 or greater magnitude in loading estimates was created in this aim. Once the factors were identified, an adjusted ordinal logistic regression analysis was conducted with the 3 identified latent variables and the CMS-RS.

\section{Least Absolute Shrinkage and Selection Operator (LASSO) Method}

Traditional stepwise selection methods, like the backward selection previously used, can experience high variability and low prediction accuracy, especially when there are many predictor variables or correlated predictor variables (or both). Therefore, the least absolute shrinkage and selection operator or LASSO penalized regression method was additionally used to explore the prediction accuracy of UM-VOC concentrations and prevalent CMS. The LASSO method shrinks the maximum likelihood estimates of model parameters toward zero by imposing an $L_{1}$ norm penalty (the sum of the absolute values of the estimates) to the log likelihood function used in parameter estimations (244). A regression model of all 21 UM-VOCs was conducted using SAS Proc GLMSELECT on the full cohort. The LASSO method was used to identify the most parsimonious model for all UM-VOCs individually in a model as well as for all 2way interactions in a model, for the best predictive model of UM-VOCs and CMS (dichotomous). The SAS Selection statement requesting a LASSO fit procedure 
was used for this analysis. The LASSO analysis method can be used as a variable selection method as the penalizations often shrink coefficients of less explanatory variables to zero within the regression model. The penalization process and subsequent coefficient shrinkage leading to fewer variables in the LASSO method also decrease overfitting.

The large sample size of the cohort allowed for the optimum model to be selected via calculating the prediction error of the models directly; using training observations $(n=2886)$ to produce the solution path and a validation set $(n=1186)$ as the tuning method by estimating the prediction error of each model on the solution path. The model with the smallest average square error (ASE) produced by the validation data was selected as the optimum model. A coefficients plot was generated, which shows how the standardized coefficients change during the selection process. The coefficients plot shows on the $x$-axis what can be interpreted as the shrinkage parameter " $\mathrm{t}$ " and the $\mathrm{y}$-axis shows the standardized coefficients for the variables.

\section{Cumulative VOC Exposure Score ( $\Sigma$ UM-VOC)}

A cumulative VOC exposure score ( $(\mathrm{UM}-\mathrm{VOC})$ was generated as a mixtures or multiple exposures-approach of assessing the association of multipollutant VOC exposures with CMS. To minimize any potential multicollinearity, the cumulative VOC exposure score was generated in the nontobacco smoke exposed participants of the cohort, and only with metabolites with greater than $40 \%$ of samples measured above the LOD. For each parent VOC, a representative metabolite was selected. If a parent VOC had multiple eligible 
metabolites, the more significant metabolite from the ordinal logistic regression models was selected as the representative. If none were significant, the metabolite with the highest abundance was selected as the representative metabolite for that VOC. The representative UM-VOCs were categorized into quintiles using cutoff points of $20_{\mathrm{th}}, 40_{\mathrm{th}}, 60_{\mathrm{th}}$, and $80_{\mathrm{th}}$ percentile values, and assigned rank values of 1 through 5, respectively. UM-VOCs at or below the LOD were forced into the lowest exposure group, the 20th percentile group.

To generate a cumulative measure for UM-VOCs, the ranks were summed for each of the representative UM-VOCs. The summary values were subsequently treated as a new continuous exposure measure with a maximum value of 65 (5 rank values $\times 13$ metabolites). The cumulative VOC exposure score was then used in survey-weighted regression models as the independent variable to assess the associations between cumulative VOC exposures and CMS. 


\section{CHAPTER THREE}

\section{RESULTS}

\section{Aim One}

Characteristics of the Study Cohort

Demographic data from 4654 participants who were at least 20 years of age, not pregnant or breastfeeding, have UM-VOC and all CMS criteria data, and had no prevalent CVD at the time of data collection are presented in Table 3.1.1 by NHANES cycle (2005-2006, 2011-2012, and 2013-2014). The means, percents, and standard errors presented in this table have all been appropriately weighted for the complex survey design of NHANES. Throughout the years of the cross-sectional NHANES cohorts used in this study, the basic sociodemographic and behavioral characteristics of the 3 cohorts had no significant change among age (mean 45.68 year), sex (female: 51.31\%, male: $48.69 \%$ ), race (non-Hispanic white: $68.51 \%$, non-Hispanic black: 10.88\%, Mexican American: 8.53\%, other Hispanic: $5.25 \%$, and other/multi-racial: $6.83 \%)$, education (15.04\% obtain less than a high school education), household income (13.58\% below $\$ 20,000$ annually), alcohol consumption (78.34\%), or physical inactivity (27.83\%).

There was a significant difference among the percent of participants 
exposed to tobacco smoke, with trends towards less exposure in recent years. A number of medical history outcomes and covariates assessed varied significantly by year. The percent of participants having type 2 diabetes mellitus, dysglycemia, and CMS as well as the mean $\mathrm{HbA} 1 \mathrm{c}$ and urinary creatinine $(\mathrm{mg} / \mathrm{dL})$ were all significantly different across NHANES waves with highest percent/means being observed in the 2011-2012 NHANES wave with the exception of urinary creatinine whose highest mean was observed in the 2005-2006 NHANES wave.

Table 3.1.1. Study Population Characteristics Stratified by NHANES Survey Cycle

\begin{tabular}{|c|c|c|c|c|c|c|c|c|c|c|c|c|c|c|}
\hline & \multicolumn{4}{|c|}{$\begin{array}{c}\text { Full Cohort } \\
\mathrm{N}=4654\end{array}$} & \multicolumn{3}{|c|}{$\begin{array}{r}2005-06 \\
n=1784\end{array}$} & \multicolumn{3}{|c|}{$\begin{array}{c}2011-12 \\
n=1380\end{array}$} & \multicolumn{3}{|c|}{$\begin{array}{c}2013-14 \\
n=1490\end{array}$} & \multirow[b]{2}{*}{ p-value } \\
\hline & $\mathrm{n}$ & $\mathrm{n}$ miss & mean $/ \%$ & SE & $\mathrm{n}$ & mean $/ \%$ & SE & $\mathrm{n}$ & mean $/ \%$ & SE & $\mathrm{n}$ & mean $/ \%$ & SE & \\
\hline Age & & 0 & 45.68 & 0.39 & & 45.23 & 0.67 & & 45.86 & 0.87 & & 46.11 & 0.40 & 0.272 \\
\hline $\operatorname{Sex}(\%)$ & & 0 & & & & & & & & & & & & 0.753 \\
\hline Female & 2351 & & 51.31 & 0.69 & 890 & 50.73 & 0.80 & 682 & 52.03 & 1.32 & 779 & 51.38 & 1.56 & \\
\hline Male & 2303 & & 48.69 & 0.69 & 894 & 49.27 & 0.80 & 698 & 47.97 & 1.32 & 711 & 48.62 & 1.56 & \\
\hline Race or ethnic origin (\%) & & 0 & & & & & & & & & & & & 0.282 \\
\hline Mexican American & 734 & & 8.53 & 0.95 & 378 & 8.12 & 1.08 & 128 & 7.38 & 1.83 & 228 & 10.20 & 2.16 & \\
\hline Other Hispanic & 338 & & 5.25 & 0.67 & 54 & 3.43 & 0.87 & 145 & 7.39 & 1.60 & 139 & 5.64 & 1.19 & \\
\hline Non-Hispanic White & 2006 & & 68.51 & 1.84 & 870 & 72.06 & 2.48 & 505 & 67.19 & 3.86 & 631 & 65.02 & 3.47 & \\
\hline Non-Hispanic Black & 1042 & & 10.88 & 1.15 & 405 & 10.83 & 1.98 & 352 & 10.94 & 2.17 & 285 & 10.88 & 1.70 & \\
\hline Other/Multi-Racial & 534 & & 6.83 & 0.49 & 77 & 5.57 & 0.73 & 250 & 7.10 & 1.00 & 207 & 8.25 & 0.87 & \\
\hline \multicolumn{15}{|l|}{ Medical History } \\
\hline $\mathrm{BMI}(\mathrm{kg} / \mathrm{m} 2)$ & & 0 & 28.58 & 0.14 & & 28.41 & 0.23 & & 28.56 & 0.30 & & 28.85 & 0.21 & 0.372 \\
\hline Overwieght (\%) & 2563 & 0 & 54.46 & 1.13 & 957 & 52.79 & 1.93 & 752 & 54.71 & 2.58 & 854 & 56.47 & 1.26 & 0.408 \\
\hline Type 2 diabetes (\%) & 460 & 0 & 7.70 & 0.47 & 164 & 6.48 & 0.78 & 154 & 9.36 & 0.66 & 142 & 7.72 & 0.87 & 0.030 \\
\hline Glycohemoglobin(\%)-HbA1c & & 0 & 5.51 & 0.02 & & 5.42 & 0.03 & & 5.61 & 0.02 & & 5.53 & 0.02 & $<.0001$ \\
\hline Dysglycemia (\%) & 1595 & 0 & 26.79 & 0.80 & 504 & 21.68 & 1.25 & 560 & 31.68 & 1.31 & 531 & 28.91 & 1.61 & $<.0001$ \\
\hline HDL-cholesterol & & 0 & & & & & & & & & & & & \\
\hline Men & & & 48.32 & 0.38 & & 48.20 & 0.46 & & 47.90 & 0.72 & & 48.87 & 0.84 & 0.676 \\
\hline Women & & & 58.65 & 0.41 & & 58.88 & 0.69 & & 58.28 & 0.80 & & 58.70 & 0.65 & 0.846 \\
\hline Low HDL-cholesterol (\%) & 495 & 0 & 10.39 & 0.68 & 175 & 9.86 & 0.88 & 144 & 10.66 & 1.55 & 176 & 10.84 & 1.20 & 0.818 \\
\hline Systolic Blood Pressure & & 0 & 121.26 & 0.37 & & 122.01 & 0.52 & & 120.44 & 0.86 & & 121.03 & 0.55 & 0.227 \\
\hline Diastolic Blood Pressure & & 0 & 70.98 & 0.34 & & 71.34 & 0.52 & & 71.37 & 0.73 & & 70.13 & 0.52 & 0.204 \\
\hline Hypertension (\%) & 1412 & 0 & 26.87 & 0.79 & 544 & 26.64 & 1.29 & 410 & 26.01 & 1.74 & 458 & 28.02 & 1.02 & 0.603 \\
\hline Albumin:Creatinine Ratio (mg/g) & & 0 & 23.93 & 2.02 & & 25.69 & 3.39 & & 21.20 & 2.65 & & 24.22 & 4.14 & 0.562 \\
\hline Microalbuminuria (\%) & 460 & 0 & 7.80 & 0.46 & 184 & 7.81 & 0.49 & 144 & 8.07 & 0.89 & 132 & 7.52 & 1.09 & 0.908 \\
\hline Urinary creatinine $(\mathrm{mg} / \mathrm{dL})$ & & 0 & 116.99 & 1.45 & & 122.56 & 2.06 & & 114.06 & 3.15 & & 112.33 & 2.39 & 0.005 \\
\hline Cardiometacolic Syndrome (\%) & 811 & 0 & 13.80 & 0.55 & 264 & 11.21 & 0.69 & 268 & 16.08 & 1.24 & 279 & 15.07 & 1.03 & 0.001 \\
\hline Blood pressure treatment (\%) & 987 & 264 & 19.84 & 0.76 & 354 & 18.65 & 1.03 & 302 & 20.41 & 1.64 & 331 & 20.89 & 1.40 & 0.437 \\
\hline Cancer $(\%)$ & 350 & 232 & 8.58 & 0.58 & 135 & 8.12 & 0.93 & 94 & 8.27 & 0.99 & 121 & 9.49 & 1.14 & 0.585 \\
\hline \multicolumn{15}{|l|}{ Lifestyle } \\
\hline$<$ High school Education (\%) & 1064 & & 15.04 & 0.97 & 470 & 15.88 & 1.48 & 288 & 14.80 & 2.07 & 306 & 14.14 & 1.52 & 0.751 \\
\hline Below $\$ 20,000 \mathrm{HH}$ income (\%) & 867 & 294 & 13.58 & 0.77 & 321 & 12.58 & 0.83 & 284 & 15.89 & 1.88 & 262 & 12.76 & 1.52 & 0.195 \\
\hline Smoking exposure (\%) & & 368 & & & & & & & & & & & & $<.0001$ \\
\hline Current smoker & 1234 & & 29.04 & 1.05 & 513 & 31.01 & 1.98 & 331 & 26.56 & 1.56 & 390 & 28.70 & 1.60 & \\
\hline Secondhand Smoke exp & 942 & & 19.83 & 0.79 & 447 & 24.69 & 1.13 & 235 & 15.17 & 1.26 & 260 & 17.52 & 1.80 & \\
\hline Non-exposed & 2110 & & 51.13 & 1.35 & 725 & 44.31 & 2.18 & 693 & 58.27 & 1.90 & 692 & 53.78 & 2.90 & \\
\hline Serum cotinine (ng/mL) & 4652 & 2 & 60.19 & 3.11 & 1784 & 62.91 & 5.02 & 1380 & 51.41 & 4.29 & 1488 & 65.08 & 6.52 & 0.118 \\
\hline Consume alcohol (\%) & 3128 & 353 & 78.34 & 1.14 & 1183 & 75.76 & 1.92 & 932 & 81.54 & 1.77 & 1013 & 78.82 & 2.24 & 0.114 \\
\hline Physically inactive (\%) & 1521 & 11 & 27.83 & 1.08 & 658 & 31.62 & 2.30 & 425 & 25.08 & 1.24 & 438 & 25.39 & 1.27 & 0.435 \\
\hline
\end{tabular}


Demographic characteristics stratified by cardiometabolic syndrome status for the 4,654 participants from the three NHANES cycles are presented in Table 3.1.2, with appropriate survey weighting variables applied. Sex and tobacco smoke exposure did not vary significantly by CMS status. However, individuals with CMS were significantly older (56.8 years) than individuals without CMS (43.9 years). A significant difference in race/ethnic origin was observed among individuals with CMS when compared to individuals without CMS ( $p<0.0001$ ); Non-Hispanic blacks had a disproportionate number of individuals with CMS. In fact, nonHispanic blacks accounted for $17.5 \%$ of individuals with CMS as compared to only $9.8 \%$ of individuals without CMS. Over a quarter of non-Hispanic blacks had CMS as compared to an average of $15.6 \%$ among all other races. Additionally, women had a slightly higher prevalence of CMS as compared to men (56.0\% vs. $44.0 \%)$ $(p=0.0718)$ and non-Hispanic black women had the highest prevalence of CMS (29.1\%) as compared to all other race/ethnic origin or sex groups (appendix Table A.1.)

Individuals with CMS had significantly unhealthier medical history outcomes and covariates as compared to individuals without CMS including all components of the CMS definition. More specifically, individuals with CMS compared to individuals without CMS had significantly higher BMI ( $\left.\mathrm{kg} / \mathrm{m}_{2}\right)$ (34.1 vs. 27.7), HbA1c (glycohemoglobin percent) (6.5 vs. 5.4), systolic blood pressure (mmHg) (132.9 vs. 119.4), diastolic blood pressure $(\mathrm{mmHg})$ (73.2 vs. 70.6), albumin:creatinine ratio (mg/g) (84.5 vs. 14.2), and lower HDL-cholesterol levels (men: 42.4 vs. 49.2 , women: 51.2 vs. 60.0 ). Individuals with CMS were more likely 
to be over weight $(94.1 \%$ vs. $48.1 \%)$, have type 2 diabetes mellitus $(39.8 \%$ vs. $2.9 \%$ ), dysglycemia ( $100 \%$ vs. $15.1 \%$ ), low HDL-cholesterol ( $24.5 \%$ vs. $8.1 \%$ ), hypertension ( $80.9 \%$ vs. $18.2 \%)$, microalbuminuria ( $25.9 \%$ vs. $4.9 \%)$ and high blood pressure (65.3\% vs. $12.5 \%)$ and nearly twice as likely to have had cancer (14.6\% vs. $7.6 \%$ ). Individuals without CMS did not have significantly higher $(\mathrm{p}=0.465)$ urinary creatinine $(\mathrm{mg} / \mathrm{dL})$ as compared to individuals with CMS (117.4 vs. $114.7, p=0.465)$. Additionally, individuals with CMS were significantly more likely to have less than a high school education $(p<0.0001)$, have a household income less than $\$ 20,000$ annually $(p=0.0017)$, and be less physically active $(p=0.0048)$. 
Table 3.1.2. Study Population Characteristics Stratified by Cardiometabolic Syndrome Status

\begin{tabular}{|c|c|c|c|c|c|c|c|c|c|c|}
\hline & \multicolumn{3}{|c|}{$\begin{array}{l}\text { Full Cohort } \\
\qquad \mathrm{N}=4654\end{array}$} & \multicolumn{3}{|c|}{$\begin{array}{c}\text { CMS } \\
n=811(17.43 \%) \\
\end{array}$} & \multicolumn{3}{|c|}{$\begin{array}{c}\text { No CMS } \\
n=3843(82.57 \%)\end{array}$} & \multirow[b]{2}{*}{ p-value } \\
\hline & $\mathrm{n}$ & mean/\% & SE & $\mathrm{n}$ & mean/\% & SE & $\mathrm{n}$ & mean/\% & SE & \\
\hline Age (years) & 4654 & 45.68 & 0.39 & 811 & 56.81 & 0.56 & 3843 & 43.90 & 0.40 & $<.0001$ \\
\hline Sex (\%) & & & & & & & & & & 0.0718 \\
\hline Female & 2351 & 51.31 & 0.69 & 460 & 56.04 & 2.62 & 1891 & 50.55 & 0.85 & \\
\hline Male & 2303 & 48.69 & 0.69 & 351 & 43.96 & 2.62 & 1952 & 49.45 & 0.85 & \\
\hline Race or ethnic origin (\%) & & & & & & & & & & $<.0001$ \\
\hline Mexican American & 734 & 8.53 & 0.95 & 136 & 8.91 & 1.59 & 598 & 8.47 & 0.92 & \\
\hline Other Hispanic & 338 & 5.25 & 0.67 & 52 & 4.34 & 0.85 & 286 & 5.40 & 0.69 & \\
\hline Non-Hispanic White & 2006 & 68.51 & 1.84 & 286 & 62.69 & 2.77 & 1720 & 69.45 & 1.79 & \\
\hline Non-Hispanic Black & 1042 & 10.88 & 1.15 & 261 & 17.54 & 2.14 & 781 & 9.81 & 1.03 & \\
\hline Other/Multi-Racial & 534 & 6.83 & 0.49 & 76 & 6.53 & 1.00 & 458 & 6.87 & 0.50 & \\
\hline \multicolumn{11}{|l|}{ Medical History } \\
\hline BMI (kg/m2) & & 28.58 & 0.14 & 808 & 34.07 & 0.38 & 3837 & 27.71 & 0.14 & $<.0001$ \\
\hline Overweight (\%) & 2563 & 54.46 & 1.13 & 745 & 94.13 & 1.14 & 1818 & 48.11 & 1.20 & $<.0001$ \\
\hline Type 2 diabetes (\%) & 460 & 7.70 & 0.47 & 308 & 39.77 & 2.06 & 152 & 2.86 & 0.31 & $<.0001$ \\
\hline $\mathrm{HbA} 1 \mathrm{c}$ & & 5.51 & 0.02 & 810 & 6.53 & 0.05 & 3843 & 5.35 & 0.01 & $<.0001$ \\
\hline Dysglycemia (\%) & 1595 & 26.79 & 0.80 & 811 & 100.00 & 0.00 & 784 & 15.07 & 0.65 & \\
\hline \multicolumn{11}{|l|}{ HDL-cholesterol } \\
\hline Men & & 48.32 & 0.38 & 351 & 42.40 & 0.96 & 1952 & 49.16 & 0.42 & $<.0001$ \\
\hline Women & & 58.65 & 0.41 & 460 & 51.16 & 0.80 & 1891 & 59.98 & 0.46 & $<.0001$ \\
\hline Low HDL-cholesterol (\%) & 495 & 10.39 & 0.68 & 187 & 24.48 & 2.23 & 308 & 8.13 & 0.70 & $<.0001$ \\
\hline Systolic Blood Pressure & & 121.26 & 0.37 & 780 & 132.85 & 1.23 & 3728 & 119.42 & 0.34 & $<.0001$ \\
\hline Diastolic Blood Pressure & & 70.98 & 0.34 & 775 & 73.24 & 0.74 & 3718 & 70.63 & 0.33 & 0.0003 \\
\hline Hypertension (\%) & 1412 & 26.87 & 0.79 & 663 & 80.93 & 2.13 & 749 & 18.21 & 0.68 & $<.0001$ \\
\hline Albumin:Creatinine Ratio & & 23.93 & 2.02 & 811 & 84.47 & 13.69 & 3843 & 14.23 & 0.99 & $<.0001$ \\
\hline Microalbuminuria (\%) & 460 & 7.80 & 0.46 & 241 & 25.92 & 2.40 & 219 & 4.90 & 0.40 & $<.0001$ \\
\hline Urinary creatinine & & 116.99 & 1.45 & 811 & 114.70 & 3.30 & 3843 & 117.35 & 1.58 & 0.465 \\
\hline Blood pressure treatment (\%) & 987 & 19.84 & 0.76 & 512 & 65.33 & 2.56 & 475 & 12.48 & 0.68 & $<.0001$ \\
\hline Ever had Cancer (\%) & 350 & 8.58 & 0.58 & 100 & 14.59 & 1.18 & 250 & 7.61 & 0.62 & $<.0001$ \\
\hline \multicolumn{11}{|l|}{ Lifestyle } \\
\hline < High school Education (\%) & 1064 & 15.04 & 0.97 & 252 & 20.76 & 1.86 & 812 & 14.13 & 0.96 & $<.0001$ \\
\hline Below $\$ 20,000 \mathrm{HH}$ income (\%) & 867 & 13.58 & 0.77 & 194 & 18.47 & 1.71 & 673 & 12.80 & 0.86 & 0.0017 \\
\hline Smoking exposure (\%) & & & & & & & & & & 0.0634 \\
\hline Current smoker & 1234 & 29.04 & 1.05 & 183 & 24.70 & 2.10 & 1051 & 29.75 & 1.17 & \\
\hline Secondhand Smoke exp & 942 & 19.83 & 0.79 & 173 & 19.32 & 2.15 & 769 & 19.91 & 0.77 & \\
\hline Non-exposed & 2110 & 51.13 & 1.35 & 397 & 55.98 & 2.21 & 1713 & 50.34 & 1.45 & \\
\hline Serum cotinine & 4652 & 60.19 & 3.11 & 811 & 54.90 & 6.12 & 3841 & 61.03 & 3.41 & \\
\hline Consume alcohol (\%) & 3128 & 78.34 & 1.14 & 479 & 69.10 & 2.43 & 2649 & 79.83 & 1.14 & $<.0001$ \\
\hline Physically inactive (\%) & 1521 & 27.83 & 1.08 & 355 & 39.17 & 2.11 & 1166 & 26.01 & 1.21 & 0.0048 \\
\hline
\end{tabular}

\section{Urinary Metabolites of Volatile Organic Compounds Characteristics}

Data from 27 urinary metabolites of volatile organic compounds (UM-VOCs) 
metabolized from 19 parent VOCs are presented in Table 3.1.3. Table 3.1.3 includes the International Union of Pure and Applied Chemistry (IUPAC) name, the abbreviation of each UM-VOC as well as the lower level of detection (LLOD) and the percent of participants with values for each UM-VOC at or above the level of detection (LOD) for the full-appended cohort as well as for each wave of NHANES assessed. Percent values for UM-VOCs at or above the LOD varied from less than $1 \%$ for N-Acetyl-S-(1,2-dichlorovinyl)-L-cysteine (1,2DCVMA) to over $99 \%$ for $\mathrm{N}$ Acetyl-S-(3-hydroxypropyl-1-methyl)-L-cysteine (ATCA). There were six UMVOCs that had less than $15 \%$ of participants with values at or above the LOD. These metabolites were two 1,3-Butadiene metabolites N-Acetyl-S-(1hydroxymethyl-2-propenyl)-L-cysteine (MHBMA1), N-Acetyl-S-(2-hydroxy-3butenyl)-L-cysteine (MHBMA2), the tetrachloroehtylene metabolite N-Acetyl-S(trichlorovinyl)-L-cysteine (TCVMA), two trichloroethylene metabolites N-Acetyl-S(1,2-dichlorovinyl)-L-cysteine $\quad(1,2 \mathrm{DCVMA}), \quad \mathrm{N}$-Acetyl-S-(2,2-dichlorovinyl)-Lcysteine (2,2DCVMA), and one xylene metabolite N-Acetyl-S-(dimethylphenyl)-Lcysteine (DPMA). These six UM-VOCs were removed from any further analyses due to unreliability of statistical tests with very limited sample sizes. Therefore, further analyses were limited to the remaining 21 UM-VOCs from 17 parent VOCs, namely, N-Acetyl-S- (2-carboxyethyl)-L-cysteine (CEMA), N-Acetyl-S- (3Hydroxypropyl)-L-cysteine (3HPMA), N-Acetyl-S-(2-carbamoylethyl)-L-cysteine (AAMA), N-Acetyl-S-(2-carbamoyl-2-hydroxyethyl)-L-cysteine (GAMA), N-AcetylS-(2-cyanoethyl)-L-cysteine (CYMA), N-Acetyl-S- (2-hydroxyethyl)-L-cysteine (HEMA), N-Acetyl-S-(phenyl)-L-cysteine (PMA), N-Acetyl-S-(n-propyl)-L-cysteine 
(BPMA), N-Acetyl-S- (3,4-dihydroxybutyl)-L-cysteine (DHBMA), N-Acetyl-S-(4hydroxy-2-buten-1-yl)-L-cysteine (MHBMA3), 2-Thioxothiazolidine-4-carboxylic acid (TTCA), N-Acetyl-S-(3-hydroxypropyl-1-methyl)-L-cysteine (HPMMA), 2Aminothiazoline-4-carboxylic acid (ATCA), N-Acetyl-S-(N-methylcarbamoyl)-Lcysteine (AMCC), Phenylglyoxylic acid (PGA), N-Acetyl-S-(2-hydroxypropyl)-Lcysteine (2HPMA), N-Acetyl-S-(1-phenyl-2-hydroxyethyl-L-cysteine + N-Acetyl-S(2-phenyl-2-hydroxyethyl)-L-cysteine (PHEMA), Mandelic acid (MA), N-Acetyl-S(benzyl)-L-cysteine (BMA), 2-Methylhippuric acid (2MHA), and 3-Methylhippuric acid + 4-Methylhippuric acid (3\&4MHA).

Table 3.1.3. Volatile Organic Compound Metabolite Level of Detection

\begin{tabular}{|c|c|c|c|c|c|c|c|}
\hline \multirow[b]{2}{*}{ Parent Compound } & \multirow[b]{2}{*}{ VOC metabolite (IUPAC name) } & \multirow[b]{2}{*}{$\begin{array}{l}\text { Metabolite } \\
\text { Abbreviation }\end{array}$} & \multirow[b]{2}{*}{$\begin{array}{l}\text { LLOD } \\
(\mathrm{ng} / \mathrm{mL})\end{array}$} & \multicolumn{4}{|c|}{ Percent at or Above LOD } \\
\hline & & & & $\begin{array}{l}\text { Appended } \\
\mathrm{N}=4654\end{array}$ & $\begin{array}{l}2005 / 06 \\
n=1784\end{array}$ & $\begin{array}{c}2011 / 12 \\
n=1380\end{array}$ & $\begin{array}{c}2013 / 14 \\
n=1490\end{array}$ \\
\hline \multirow[t]{2}{*}{ Acrolein } & N-Acetyl-S- (2-carboxyethyl)-L-cysteine & CEMA & 6.96 & 98.62 & 97.94 & 99.26 & 98.83 \\
\hline & N-Acetyl-S- (3-Hydroxypropyl)-L-cysteine & 3НPMA & 13.0 & 99.41 & 98.63 & 99.93 & 99.86 \\
\hline \multirow[t]{2}{*}{ Acrylamide } & N-Acetyl-S-(2-carbamoylethyl)-L-cysteine & AAMA & 2.20 & 99.49 & 98.86 & 99.93 & 99.86 \\
\hline & $\begin{array}{l}\text { N-Acetyl-S-(2-carbamoyl-2-hydroxyethyl)-L- } \\
\text { cysteine }\end{array}$ & GAMA & 9.40 & 47.93 & 58.60 & 43.26 & 39.54 \\
\hline Acrylonitrile & N-Acetyl-S-(2-cyanoethyl)-L-cysteine & CYMA & 0.500 & 90.09 & 89.82 & 90.64 & 89.90 \\
\hline $\begin{array}{l}\text { Acrylonitrile, vinyl } \\
\text { chloride, ethylene }\end{array}$ & N-Acetyl-S- (2-hydroxyethyl)-L-cysteine & HEMA & 0.791 & 40.63 & 27.16 & 54.97 & 43.47 \\
\hline Benzene & N-Acetyl-S-(phenyl)-L-cysteine & PMA & 0.600 & 32.42 & 20.64 & 44.58 & 35.30 \\
\hline 1-Bromopropane & N-Acetyl-S-( $n$-propyl)-L-cysteine & BPMA & 1.20 & 73.02 & 70.50 & 79.33 & 70.33 \\
\hline \multirow[t]{4}{*}{ 1,3-Butadiene } & N-Acetyl-S- (3,4-dihydroxybutyl)-L-cysteine & DHBMA & 5.25 & 99.76 & 99.43 & 99.93 & 100.00 \\
\hline & $\begin{array}{l}\mathrm{N} \text {-Acetyl-S-(1-hydroxymethyl-2-propenyl)-L- } \\
\text { cysteine }\end{array}$ & MHBMA1 & 0.700 & $0.88^{*}$ & 0.69 & 1.25 & 0.76 \\
\hline & N-Acetyl-S-(2-hydroxy-3-butenyl)-L-cysteine & MHBMA2 & 0.700 & $14.08^{*}$ & 15.61 & 12.82 & 13.42 \\
\hline & $\begin{array}{l}\mathrm{N} \text {-Acetyl-S-(4-hydroxy-2-buten-1-yl)-L- } \\
\text { cysteine }\end{array}$ & MHBMA3 & 0.600 & 97.51 & 96.91 & 97.27 & 98.49 \\
\hline Carbon-disulfide & 2-Thioxothiazolidine-4-carboxylic acid & TTCA & 11.2 & 38.38 & 39.62 & 38.61 & 36.69 \\
\hline Crotonaldehyde & $\begin{array}{l}\text { N-Acetyl-S-(3-hydroxypropyl-1-methyl)-L- } \\
\text { cysteine }\end{array}$ & HPMMA & 2.00 & 99.93 & 99.83 & 100.00 & 100.00 \\
\hline Cyanide & 2-Aminothiazoline-4-carboxylic acid & ATCA & 15.0 & 85.45 & 69.58 & 96.32 & 94.72 \\
\hline $\begin{array}{l}N, N- \\
\text { Dimethylformamide }\end{array}$ & $\mathrm{N}$-Acetyl-S-(N-methylcarbamoyl)-L-cysteine & AMCC & 6.26 & 99.28 & 98.34 & 99.85 & 99.86 \\
\hline $\begin{array}{l}\text { Ethylbenzene, } \\
\text { styrene }\end{array}$ & Phenylglyoxylic acid & PGA & 12.0 & 92.99 & 83.70 & 98.97 & 98.67 \\
\hline Propylene oxide & N-Acetyl-S-(2-hydroxypropyl)-L-cysteine & 2HPMA & 5.30 & 93.89 & 93.08 & 95.06 & 93.77 \\
\hline \multirow[t]{2}{*}{ Styrene } & $\begin{array}{l}\mathrm{N} \text {-Acetyl-S-(1-phenyl-2-hydroxyethyl-L- } \\
\text { cysteine }+N \text {-Acetyl-S-(2-phenyl-2- } \\
\text { hydroxyethyl)-L-cysteine }\end{array}$ & PHEMA & 0.700 & 24.97 & 8.46 & 34.41 & 36.17 \\
\hline & Mandelic acid & MA & 12.0 & 98.52 & 98.63 & 98.82 & 98.09 \\
\hline Tetrachloroethylene & N-Acetyl-S-(trichlorovinyl)-L-cysteine & TCVMA & 3.00 & $0.07^{*}$ & 0.11 & 0.07 & 0.00 \\
\hline Toluene & N-Acetyl-S-(benzyl)-L-cysteine & BMA & 0.500 & 99.12 & 99.09 & 99.26 & 99.04 \\
\hline \multirow[t]{2}{*}{ Trichloroethylene } & $\mathrm{N}$-Acetyl-S-(1,2-dichlorovinyl)-L-cysteine & $1,2 \mathrm{DCVMA}$ & 12.6 & $0.04^{*}$ & 0.06 & 0.00 & 0.07 \\
\hline & N-Acetyl-S-(2,2-dichlorovinyl)-L-cysteine & 2,2DCVMA & 4.70 & $0.35^{\star}$ & 0.69 & 0.29 & 0.00 \\
\hline \multirow[t]{3}{*}{ Xylene } & N-Acetyl-S-(dimethylphenyl)-L-cysteine & DPMA & 0.500 & $0.31^{*}$ & 0.29 & 0.29 & 0.36 \\
\hline & 2-Methylhippuric acid & $2 \mathrm{MHA}$ & 5.00 & 93.82 & 94.28 & 95.43 & 91.56 \\
\hline & 3-Methylhippuric acid + 4-Methylhippuric acid & $3 \& 4 \mathrm{MHA}$ & 8.00 & 99.54 & 99.31 & 99.93 & 99.45 \\
\hline
\end{tabular}

†App- Appended Full Cohort, LLOD- lower level of detection, *UM-VOC with less than 15\% above LOD 
To ensure that the cut-point ( $15 \%$ at or above the LOD) wasn't driven by the nearly $50 \%$ of participants who were exposed to tobacco smoke, Table 3.1 .3 was stratified by tobacco smoke exposure status (Appendix Table A.2). Upon stratification there were no additional UM-VOCs that had significantly lower values for the percent of measurements at or above the LOD and therefore the previously identified 21 UM-VOCs from 17 parent VOCs were maintained for further analysis.

The descriptive statistics for the 21-creatinine normalized UM-VOCs from 17 parent VOCs including geometric mean, standard error, minimum, quartile one, median, quartile three, and maximum values from the full-appended cohort are presented in Table 3.1.4. Table A.3 further stratifies Table 3.1.4 by NHANES wave. There were 12 UM-VOCs with significant differences between the NHANES cycles (2005-06, 2011-12, 2013-14). All significant differences observed in UM-VOCs between the NHANES waves were significant increases in UM-VOCs throughout the years, except a metabolite of propylene oxide, 2HPMA ( $p$-value=0.0287), which decreased from a geometric mean of $38.3 \mathrm{ng} / \mathrm{mg}$ creatinine in the $2005-06$ NHANES cycle to a geometric mean of $31.5 \mathrm{ng} / \mathrm{mg}$ creatinine in the $2013-14$ NHANES cycle. 
Table 3.1.4. Description of Volatile Organic Compound Metabolites for Appended NHANES Study Population

\begin{tabular}{|c|c|c|c|c|c|c|c|c|}
\hline \multirow[b]{2}{*}{ Parent Compound } & \multirow[b]{2}{*}{ Metabolite } & \multicolumn{7}{|c|}{ Appended Full Cohort ( $N=4654)$} \\
\hline & & GM & SE & Min & Q1 & Median & Q3 & Max \\
\hline \multirow[t]{2}{*}{ Acrolein } & CEMA & 93.97 & 2.20 & 1.02 & 54.41 & 90.00 & 157.02 & 4352.63 \\
\hline & 3HPMA & 259.47 & 7.13 & 1.90 & 130.31 & 214.95 & 450.64 & 8782.05 \\
\hline \multirow[t]{2}{*}{ Acrylamide } & AAMA & 55.49 & 1.29 & 0.32 & 32.47 & 51.48 & 90.91 & 1518.75 \\
\hline & GAMA & 12.83 & 0.23 & 1.37 & 7.92 & 12.55 & 20.38 & 168.57 \\
\hline Acrylonitrile & CYMA & 4.39 & 0.21 & 0.07 & 1.04 & 1.78 & 12.22 & 1623.08 \\
\hline $\begin{array}{l}\text { Acrylonitrile, vinyl chloride, } \\
\text { ethylene oxide }\end{array}$ & HEMA & 1.05 & 0.03 & 0.10 & 0.51 & 0.96 & 1.93 & 44.63 \\
\hline Benzene & PMA & 0.66 & 0.02 & 0.08 & 0.37 & 0.64 & 1.10 & 21.43 \\
\hline 1-Bromopropane & BPMA & 4.34 & 0.12 & 0.18 & 1.62 & 4.01 & 10.34 & 2288.89 \\
\hline \multirow[t]{2}{*}{ 1,3-Butadiene } & DHBMA & 275.63 & 6.39 & 0.77 & 210.55 & 279.20 & 371.51 & 3800.00 \\
\hline & МHBMA3 & 6.61 & 0.21 & 0.09 & 3.10 & 5.32 & 10.99 & 328.42 \\
\hline Carbon-disulfide & TTCA & 15.36 & 0.32 & 1.43 & 7.26 & 13.38 & 27.14 & 2140.74 \\
\hline Crotonaldehyde & HPMMA & 478.45 & 13.50 & 0.29 & 248.10 & 366.35 & 741.47 & 19421.00 \\
\hline Cyanide & ATCA & 90.29 & 3.62 & 1.92 & 46.87 & 111.12 & 216.57 & 2179.41 \\
\hline$N, N$ - Dimethylformamide & AMCC & 164.95 & 4.54 & 0.92 & 88.16 & 161.33 & 301.14 & 3578.95 \\
\hline Ethylbenzene, styrene & PGA & 149.80 & 4.30 & 1.76 & 110.01 & 177.67 & 259.90 & 55667.00 \\
\hline Propylene oxide & 2HPMA & 36.03 & 1.05 & 0.77 & 19.06 & 31.61 & 60.66 & 28130.00 \\
\hline \multirow[t]{2}{*}{ Styrene } & PHEMA & 0.73 & 0.01 & 0.09 & 0.39 & 0.67 & 1.24 & 665.56 \\
\hline & MA & 141.71 & 2.95 & 1.76 & 95.00 & 135.17 & 204.80 & 31000.00 \\
\hline Toluene & BMA & 7.08 & 0.14 & 0.07 & 3.99 & 6.46 & 11.45 & 7144.14 \\
\hline \multirow[t]{2}{*}{ Xylene } & $2 \mathrm{MHA}$ & 37.16 & 1.14 & 0.73 & 16.66 & 35.59 & 80.63 & 18384.00 \\
\hline & $3 \& 4 \mathrm{MHA}$ & 258.20 & 6.82 & 1.17 & 113.21 & 225.55 & 583.49 & 102459.00 \\
\hline
\end{tabular}

$\mathrm{GM}$ - geometric mean. Values are $\mathrm{ng} / \mathrm{mg}$ creatinine

UM-VOCs stratified by CMS status are presented in Table 3.1.5. Eight of the 21 UM-VOCs were significantly different between individuals with CMS and individuals without CMS. Four UM-VOCs were significantly higher in individuals with CMS: one acrolein metabolite CEMA, a metabolite of 1,3-butadiene DHBMA, one cyanide metabolite ATCA, and a metabolite of ethylbenzene/styrene PGA. Four UM-VOCs were significantly lower in individuals with CMS: a metabolite of acrylamide AAMA, two metabolites of acrylonitrile CYMA and HEMA, and a metabolite of xylene 2MHA. 
Table 3.1.5. Description of Volatile Organic Compound Metabolites Stratified by Cardiometabolic Syndrome Status

\begin{tabular}{|c|c|c|c|c|c|c|c|c|c|c|c|c|c|c|c|c|}
\hline \multirow[b]{2}{*}{ Parent Compound } & \multirow[b]{2}{*}{ Metabolite } & \multicolumn{7}{|c|}{ CMS $(n=811)$} & \multicolumn{7}{|c|}{ NonCMS $(n=3843)$} & \multirow[b]{2}{*}{ p-value } \\
\hline & & GM & $\mathrm{SE}$ & Min & Q1 & Median & Q3 & $\operatorname{Max}$ & GM & SE & Min & Q1 & Median & Q3 & $\operatorname{Max}$ & \\
\hline \multirow[t]{2}{*}{ Acrolein } & CEMA & 112.85 & 5.13 & 2.54 & 68.22 & 103.48 & 181.70 & 1538.46 & 91.29 & 2.17 & 1.02 & 52.99 & 86.32 & 153.75 & 4352.63 & $<.0001$ \\
\hline & 3HPMA & 260.15 & 12.51 & 6.81 & 142.27 & 217.31 & 406.96 & 7205.13 & 259.36 & 7.58 & 1.90 & 128.45 & 214.42 & 458.26 & 8782.05 & 0.9519 \\
\hline \multirow[t]{2}{*}{ Acrylamide } & AAMA & 51.43 & 2.21 & 1.16 & 31.15 & 48.88 & 81.65 & 616.67 & 56.16 & 1.31 & 0.32 & 32.80 & 51.95 & 91.53 & 1518.75 & 0.0308 \\
\hline & GAMA & 12.52 & 0.37 & 2.00 & 8.15 & 12.07 & 18.47 & 162.05 & 12.88 & 0.25 & 1.37 & 7.91 & 12.69 & 20.78 & 168.57 & 0.3827 \\
\hline Acrylonitrile & CYMA & 3.59 & 0.33 & 0.22 & 1.08 & 1.70 & 4.55 & 1623.08 & 4.53 & 0.23 & 0.07 & 1.04 & 1.81 & 14.72 & 1247.37 & 0.0159 \\
\hline $\begin{array}{l}\text { Acrylonitrile, vinyl } \\
\text { chloride, ethylene } \\
\text { oxide }\end{array}$ & HEMA & 0.97 & 0.05 & 0.10 & 0.50 & 0.90 & 1.63 & 38.43 & 1.07 & 0.03 & 0.12 & 0.51 & 0.97 & 1.99 & 44.63 & 0.0336 \\
\hline Benzene & PMA & 0.66 & 0.03 & 0.08 & 0.37 & 0.61 & 0.99 & 14.44 & 0.66 & 0.02 & 0.09 & 0.37 & 0.65 & 1.11 & 21.43 & 0.8877 \\
\hline 1-Bromopropane & BPMA & 4.45 & 0.27 & 0.25 & 1.60 & 4.22 & 9.60 & 369.85 & 4.32 & 0.13 & 0.18 & 1.63 & 3.95 & 10.45 & 2288.89 & 0.6542 \\
\hline \multirow[t]{2}{*}{ 1,3-Butadiene } & DHBMA & 299.47 & 9.61 & 2.75 & 233.98 & 304.29 & 402.59 & 2294.87 & 272.02 & 6.45 & 0.77 & 206.96 & 276.35 & 366.56 & 3800.00 & 0.0011 \\
\hline & MHBMA3 & 6.75 & 0.34 & 0.31 & 3.43 & 5.49 & 10.30 & 255.81 & 6.59 & 0.22 & 0.09 & 3.03 & 5.29 & 11.25 & 328.42 & 0.6286 \\
\hline Carbon-disulfide & TTCA & 16.55 & 0.87 & 1.43 & 7.11 & 14.22 & 26.72 & 1169.01 & 15.18 & 0.35 & 1.64 & 7.28 & 13.23 & 27.17 & 2140.74 & 0.1360 \\
\hline Crotonaldehyde & HPMMA & 514.44 & 23.92 & 2.11 & 285.93 & 397.75 & 765.52 & 16462.00 & 472.95 & 13.77 & 0.29 & 243.97 & 360.71 & 730.45 & 19421.00 & 0.0644 \\
\hline Cyanide & ATCA & 105.12 & 5.48 & 1.92 & 57.56 & 125.47 & 226.45 & 1121.43 & 88.15 & 4.00 & 2.19 & 44.55 & 109.04 & 211.73 & 2179.41 & 0.0130 \\
\hline $\begin{array}{l}\mathrm{N}, \mathrm{N}- \\
\text { Dimethylformamide }\end{array}$ & AMCC & 171.63 & 7.36 & 3.28 & 92.15 & 162.58 & 296.04 & 2507.14 & 163.91 & 4.67 & 0.92 & 87.42 & 160.99 & 301.16 & 3578.95 & 0.2629 \\
\hline Ethylbenzene, styrene & PGA & 175.84 & 6.82 & 4.59 & 126.84 & 185.68 & 272.58 & 32525.00 & 146.01 & 4.68 & 1.76 & 106.32 & 176.05 & 256.50 & 55667.00 & 0.0003 \\
\hline Propylene oxide & 2HPMA & 35.32 & 2.04 & 1.94 & 19.38 & 31.18 & 56.08 & 5421.05 & 36.15 & 1.10 & 0.77 & 19.03 & 31.71 & 60.88 & 28130.00 & 0.6921 \\
\hline \multirow[t]{2}{*}{ Styrene } & PHEMA & 0.72 & 0.03 & 0.09 & 0.39 & 0.66 & 1.17 & 555.56 & 0.73 & 0.01 & 0.10 & 0.39 & 0.68 & 1.24 & 665.56 & 0.7112 \\
\hline & MA & 142.69 & 5.19 & 4.67 & 94.43 & 133.59 & 207.03 & 27778.00 & 141.56 & 3.15 & 1.76 & 95.11 & 135.59 & 204.58 & 31000.00 & 0.8367 \\
\hline Toluene & BMA & 7.19 & 0.25 & 0.26 & 4.17 & 6.75 & 12.15 & 216.67 & 7.06 & 0.14 & 0.07 & 3.96 & 6.44 & 11.33 & 7144.14 & 0.6203 \\
\hline \multirow[t]{2}{*}{ Xylene } & $2 \mathrm{MHA}$ & 33.06 & 2.53 & 1.32 & 14.89 & 30.20 & 68.04 & 18384.00 & 37.86 & 1.04 & 0.73 & 16.77 & 36.42 & 81.82 & 1537.31 & 0.0469 \\
\hline & $3 \& 4 \mathrm{MHA}$ & 239.87 & 12.96 & 11.55 & 109.32 & 193.06 & 520.73 & 102459.00 & 261.24 & 6.70 & 1.17 & 114.09 & 233.17 & 593.97 & 12456.00 & 0.0835 \\
\hline
\end{tabular}

Among the tobacco smoke exposed study population, CEMA $(p=0.0208)$ and PGA ( $p=0.0203)$, metabolites of acrolein and ethlybenzene/styrene, respectively, were significantly higher in individuals exposed to tobacco smoke with CMS as compared to individuals exposed to tobacco smoke without CMS (Tablem3.1.6). AAMA ( $p=0.0403)$ a metabolite of acrylamide was significantly lower in individuals exposed to tobacco smoke with CMS as compared to individuals exposed to tobacco smoke without CMS. Amongst the non-tobacco smoke exposed inindividuals the study population all six UM-VOCs that were significantly different were higher in individuals exposed to tobacco smoke with CMS (CEMA: $p<0.0001$, DHBMA: $p=0.0010$, MHBMA3: $p=0.0424$, HPMMA: $p=0.0034$, ATCA: $p=0.0075$, PGA: $p=0.0003$ ) (Table 3.1.6). 
Table 3.1.6 Description of Volatile Organic Compound Metabolites with Significant Differences Based on CMS Status Stratified by Tobacco Smoke Exposure

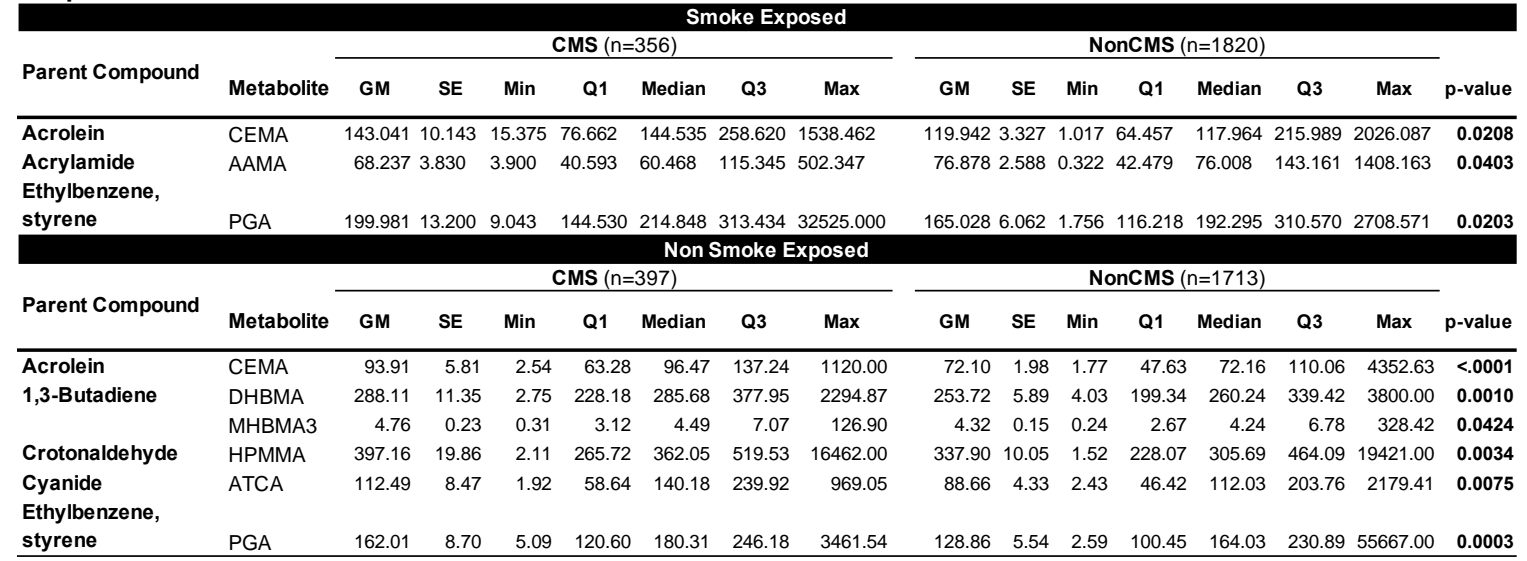

Note: GM- geometric mean. Values are $\mathrm{ng} / \mathrm{mg}$ creatinine. Tobacco smoke exposed defined using serum cotinine values $(n=4652)$ and questionnaire responses $(n=4286)$.

Correlation matrices revealed an abundance of moderate correlations; correlation coefficient $r=0.3-0.7$, for creatinine normalized log transformed UMVOCs. A few highly correlated UM-VOCs were observed; correlation coefficient $r>0.7$. The highly correlated creatinine normalized log transformed UM-VOCs for the full study group were the two metabolites of xylene MHA2 and 3\&4MHA ( $r=0.841$ ), followed by MHBMA3 a metabolite of 1,3-butadiene and HPMMA and metabolite of crotonaldehyde $(r=0.840)$, MHBMA3 and CYMA a metabolite of acrylonitrile $(r=0.800)$, and MHBMA3 and HPMA3 a metabolite of acrolein $(r=0.798)$. The least correlated UM-VOCs were in the full study group were $3 \& 4 \mathrm{MHA}$ and BPMA a metabolite of 1 -bromopropane $(r=0.003)$, AMCC a metabolite of $\mathrm{N}, \mathrm{N}$-dimethylformamide and BPMA $(\mathrm{r}=0.015)$, and $3 \& 4 \mathrm{MHA}$ and TTCA a metabolite of carbon-disulfide ( $r=0.032)$. Additionally, a very weak inverse correlation was observed between BPMA a metabolite of 1-bromopropane and CYMA a metabolite of acrylonitrile $(r=-0.008)$ (Figure 3.1.1). Tobacco smoke 
exposed individuals had many more correlated UM-VOCs than non-tobacco tobacco smoke exposed individuals (Figure A1 and Figure A2).

Theoretically, metabolites from different parent VOCs may be correlated without the parent VOCs themselves being correlated. These correlations may reflect common sources and/or metabolic pathways. Disentangling the effects of highly correlated UM-VOCs on outcomes may be challenging and therefore important to identify (Table A.4 Figure 3.1.1, Figure A.1., and Figure A.2.).

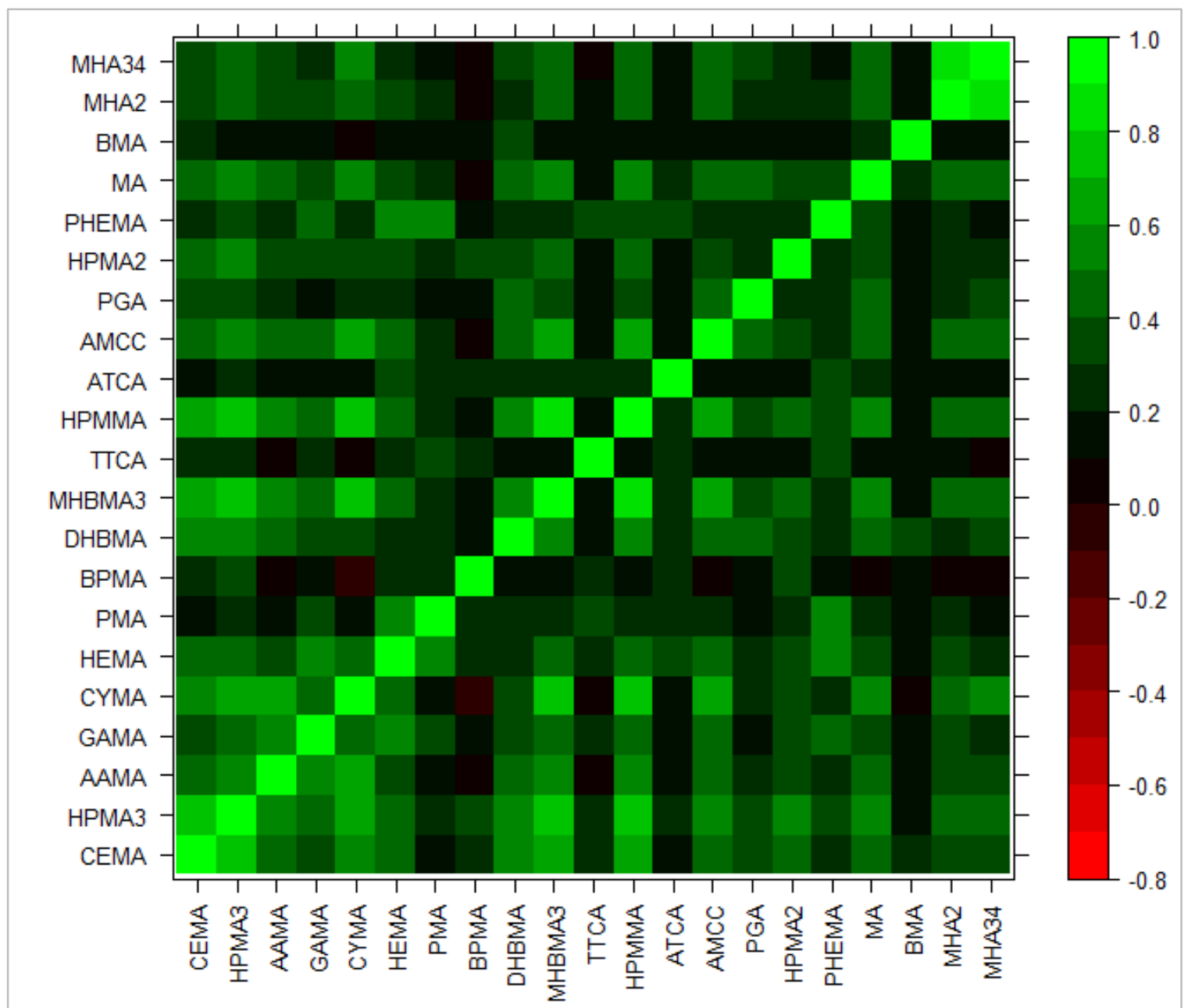

Figure 3.1.1. Correlations of each UM-VOC with each UM-VOC in the Full Study Cohort. 


\section{Aim Two}

UM-VOCs Associated with CMS

UM-VOCs were individually assessed to identify if particular metabolites were associated with CMS status using survey-weighted logistic regression models. Figure 3.2.1 shows the forest plot of the unadjusted odds ratios and $95 \%$ confidence intervals for each metabolite for the full study cohort. Overall, eight metabolites were significantly associated with CMS prevalence. Four UM-VOCs (CEMA- acrolein, DHBMA-1,3-butadiene, ATCA- cyanide, and PGAethylbenzene/styrene) were associated with significant increases in the odds of CMS, while 4 UM-VOCs (AAMA-acrylamide, CYMA-acrylonitrile, PMA- benzene, 2MHA- xylene) were associated with a significant decrease in the odds of CMS (Table A.5).

To reduce the likelihood of a type I error, a multiple comparisons adjustment was calculated and a new significance threshold was identified. The multiple comparisons adjusted $p$-value was calculated by dividing alpha, 0.05 , by the number of factors that were identified in the factor analysis. Based on the factor analysis, three latent variables were identified from the VOC metabolites. The multiple comparisons adjusted $p$-value threshold is $<0.0167$.

After adjustment for multiple comparisons, the full cohort unadjusted models showed 4 odds ratios of UM-VOCs associated with CMS (dichotomous) remained significant (CEMA OR: 1.35, 95\%Cl, 1.19-1.54; DHBMA OR: 1.44, 95\%Cl, 1.15-1.81; ATCA OR: 1.13, 95\%Cl, 1.03-1.24; PGA OR: 1.24 95\%Cl, 1.11 1.37) (Figure 3.2.1 and Table A.5). 
When stratified by smoking status, the non-tobacco smoke exposed cohort had 6 metabolites significantly positively associated with CMS (CEMA, DHBMA, MHBMA3, HPMMA, ATCA and PGA) (Figure 3.2.2 and Table A.6), while tobacco smoke exposed participants showed 3 metabolites significantly associations with CMS; two were positively associated with CMS (CEMA and PGA) and 1 negatively associated with CMS (AAMA) (Figure 3.2.3 and Table A.6)

To further investigate the UM-VOC and CMS relationship, logistic regression models adjusted for age, NHANES cohort year, and a race and sex interaction term were used. 5 UM-VOCs were associated with CMS in the full cohort fully adjusted models (Figure 3.2.4 and Table A5). Two UM-VOCs (CEMAacrolein and PGA-ethylbenzene/styrene) were significantly associated with increased odds of CMS (Figure 3.2.4). Three UM-VOCs (HEMA-acrylonitrile/vinyl chloride/ethylene oxide, PHEMA- styrene, and 3MHA/4MHA-xylene) were significantly associated with decreased odds of CMS. After adjustment for multiple comparisons, the cull cohort fully adjusted models showed 2 adjusted odds ratios of UM-VOCs associated with the prevalence of CMS remained significant (CEMA OR: 1.24, 95\%Cl: 1.08-1.43; PGA OR: 1.20, 95\%Cl: 1.06-1.37) (Table A.5). The odds ratios associated with a decreased risk did not maintain statistical significance at the multiple comparisons $p$-value threshold.

Figures 3.2.5 and Figure 3.2.6 display fully adjusted logistic regression models stratified by tobacco smoke exposure status. Among the non-tobacco smoke exposure fully adjusted models, 7 metabolites were significantly associated with CMS. Again, CEMA-acrolein and PGA-ethylbenzene/styrene were 
significantly associated with increased odds of CMS. Decreased odds of CMS were associated with HEMA-acrylonitrile/vinyl chloride/ethylene oxide, PMAbenzene, PHEMA- styrene, 2MHA-xylene, and 3MHA/4MHA-xylene (Figure 3.2.5 and Table A.7). After adjustment for multiple comparisons, none of the adjusted odds ratios of UM-VOCs associated with the prevalence of CMS remained significant among non-tobacco smoke expose participants. Among the smoke exposed individuals, zero UM-VOC were significantly associated with CMS (Figure 3.2.6). 


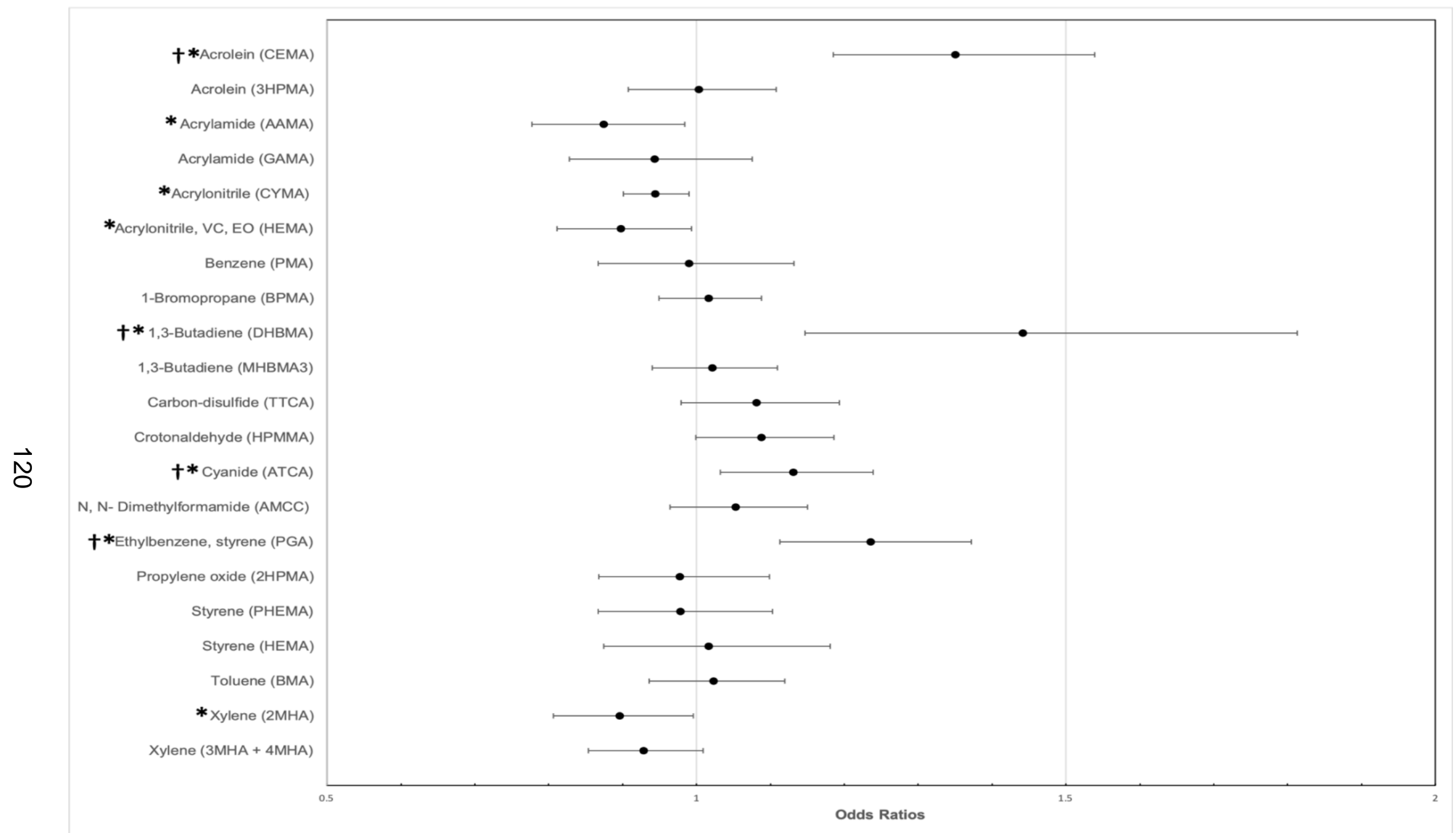

Figure 3.2.1. Forest Plot- Full Study Cohort Unadjusted Odds And 95\% CI For 21 UM-VOCs and CMS (Dichotomous) Note: ${ }^{*}=$ significance at a $p$-value threshold of $<0.05 ; \dagger=$ significant at a $p$-value threshold of $<0.0167$ 


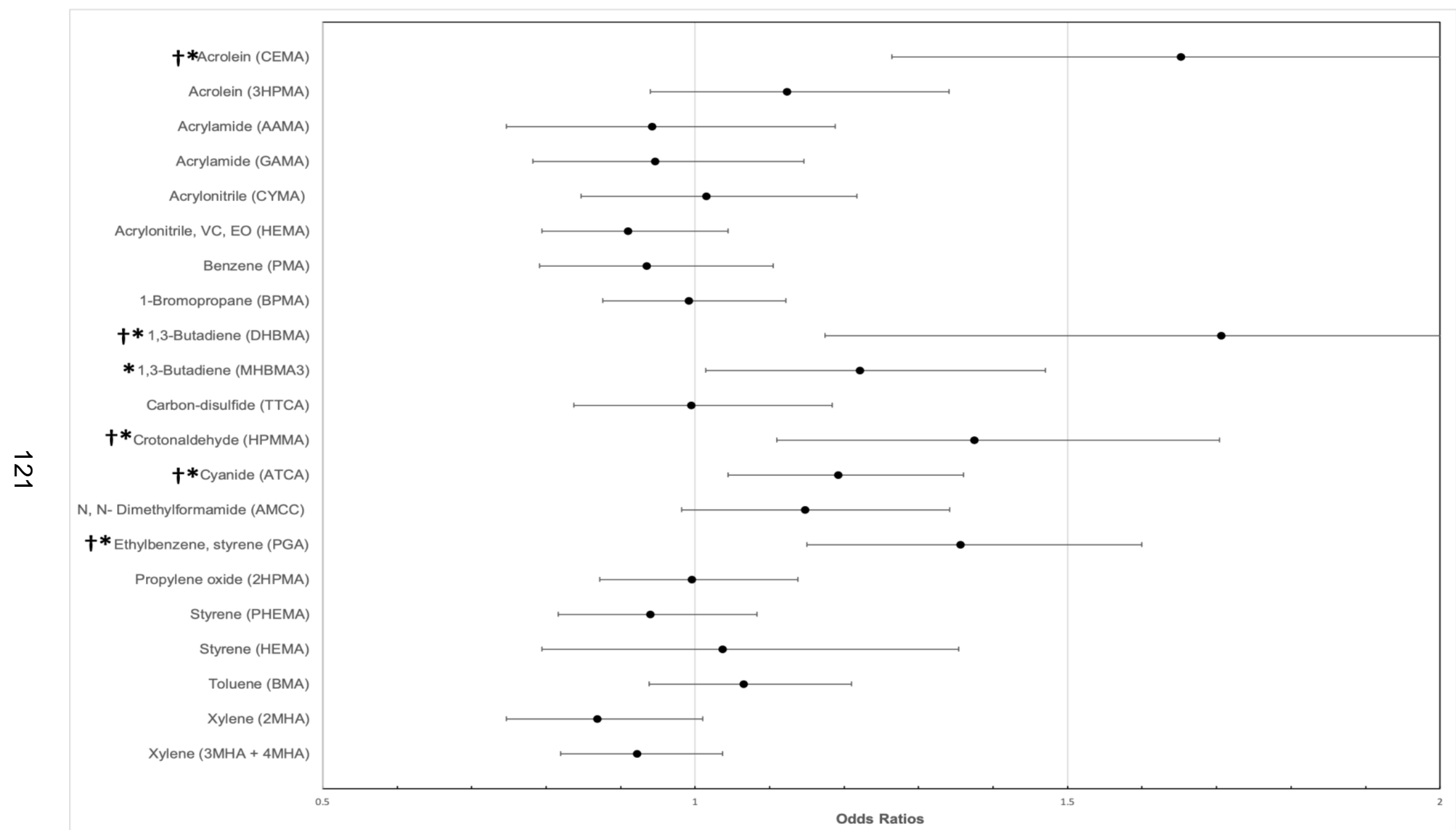

Figure 3.2.2. Forest Plot- Non-Tobacco Smoke Exposed Sub-Population, Unadjusted Odds And 95\% CI For 21 UM-VOCs and CMS (Dichotomous)

Note: ${ }^{*}=$ significance at a $p$-value threshold of $<0.05 ; \dagger=$ significant at a $p$-value threshold of $<0.0167$ 


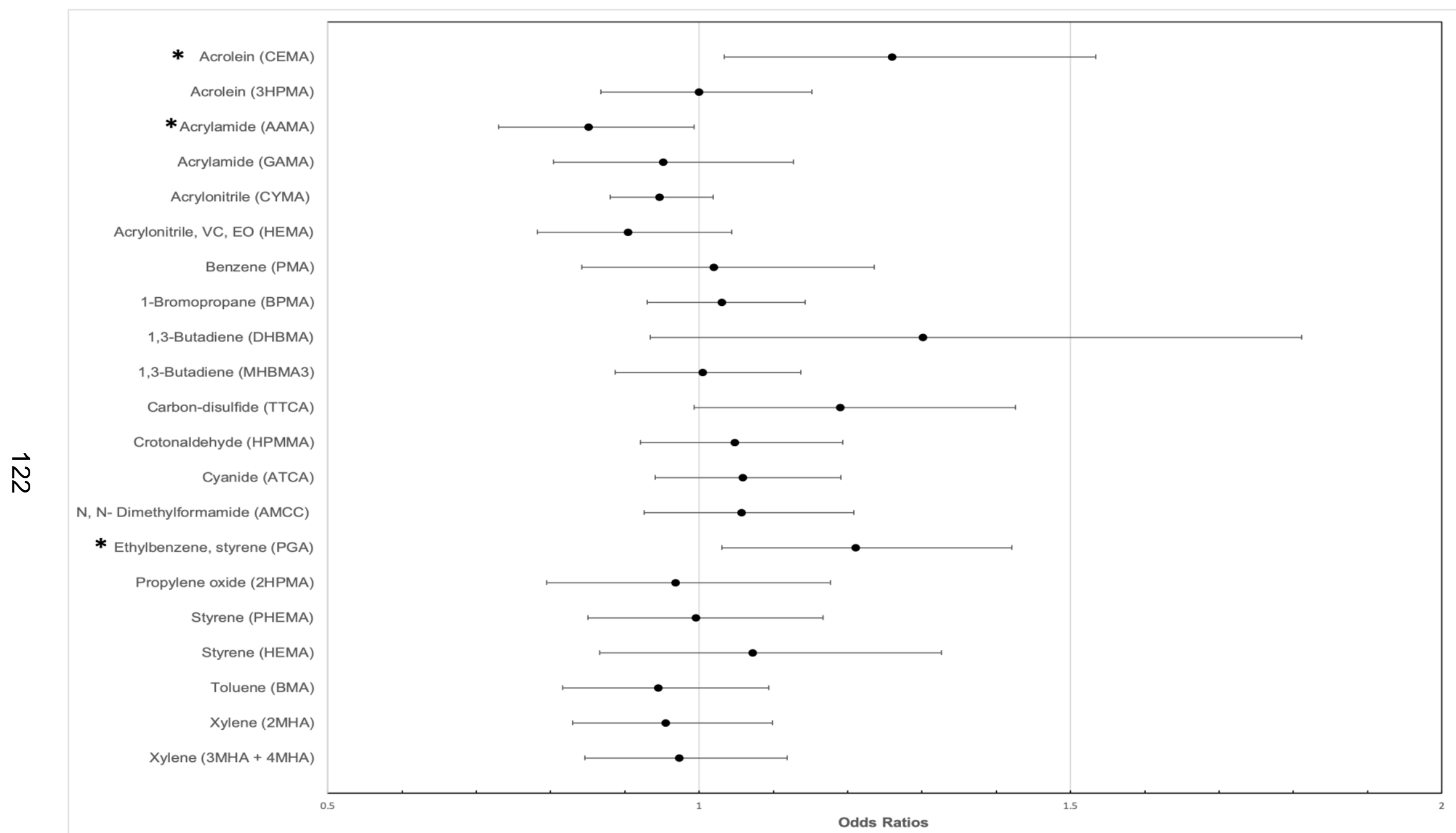

Figure 3.2.3. Forest Plot-Tobacco Smoke Exposed Sub-Population, Unadjusted Odds And 95\% Cl For 21 UM-VOCs and CMS (Dichotomous)

Note: ${ }^{\star}=$ significance at a $p$-value threshold of $<0.05 ; \dagger=$ significant at a $p$-value threshold of $<0.0167$ 


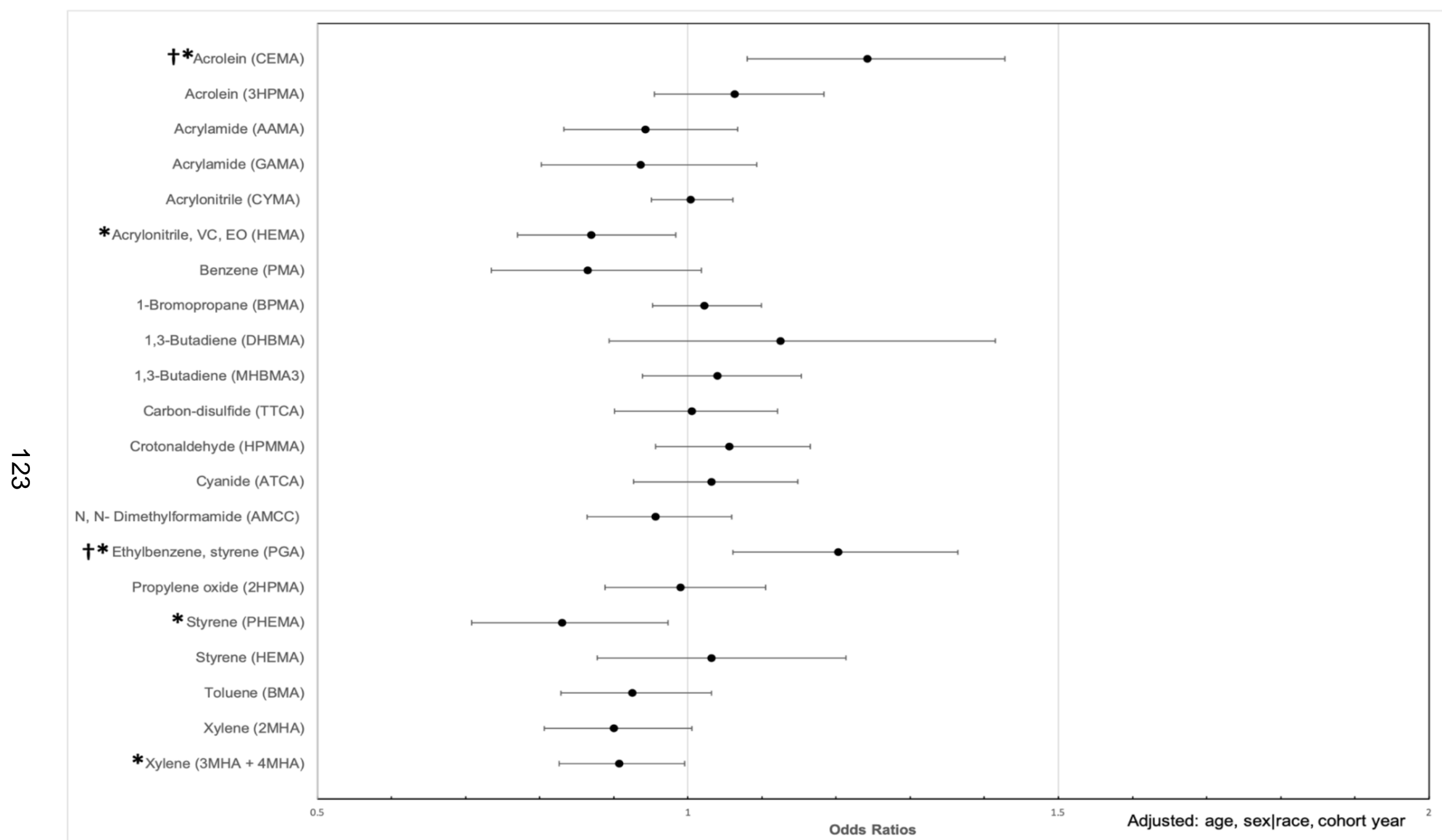

Figure 3.2.4. Forest Plot- Full Cohort, Adjusted Odds and 95\% Cl for $21 \mathrm{UM}-\mathrm{VOCs}$ and CMS (Dichotomous) Note: ${ }^{*}=$ significance at a $p$-value threshold of $<0.05 ; \dagger=$ significant at a $p$-value threshold of $<0.0167$ 


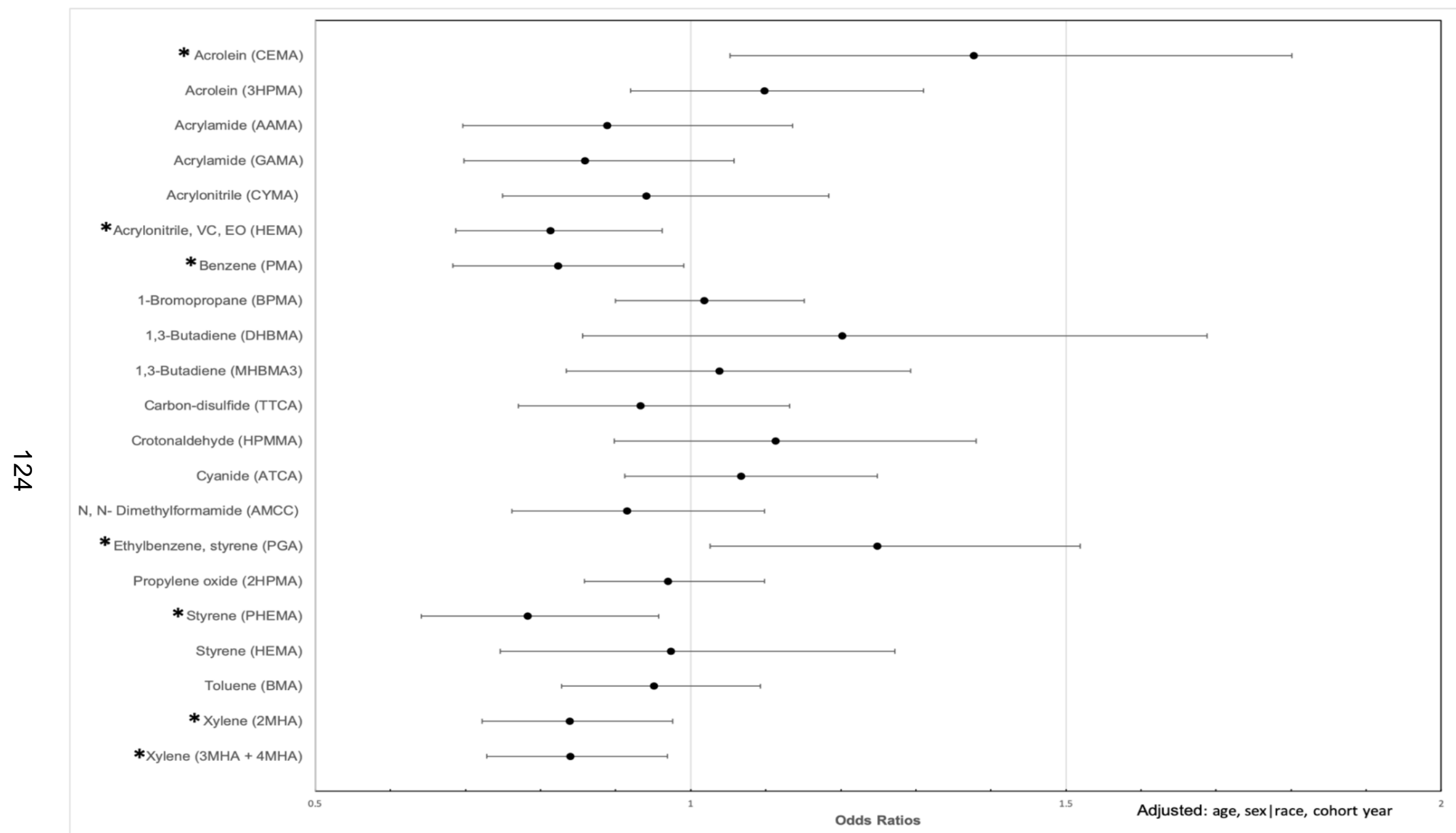

Figure 3.2.5. Forest Plot- Non-Tobacco Smoke Exposed Sub-Population, Adjusted Odds And 95\% CI For 21 UM-VOCs and CMS (Dichotomous)

Note: ${ }^{*}=$ significance at a $p$-value threshold of $<0.05 ; \dagger=$ significant at a $p$-value threshold of $<0.0167$ 


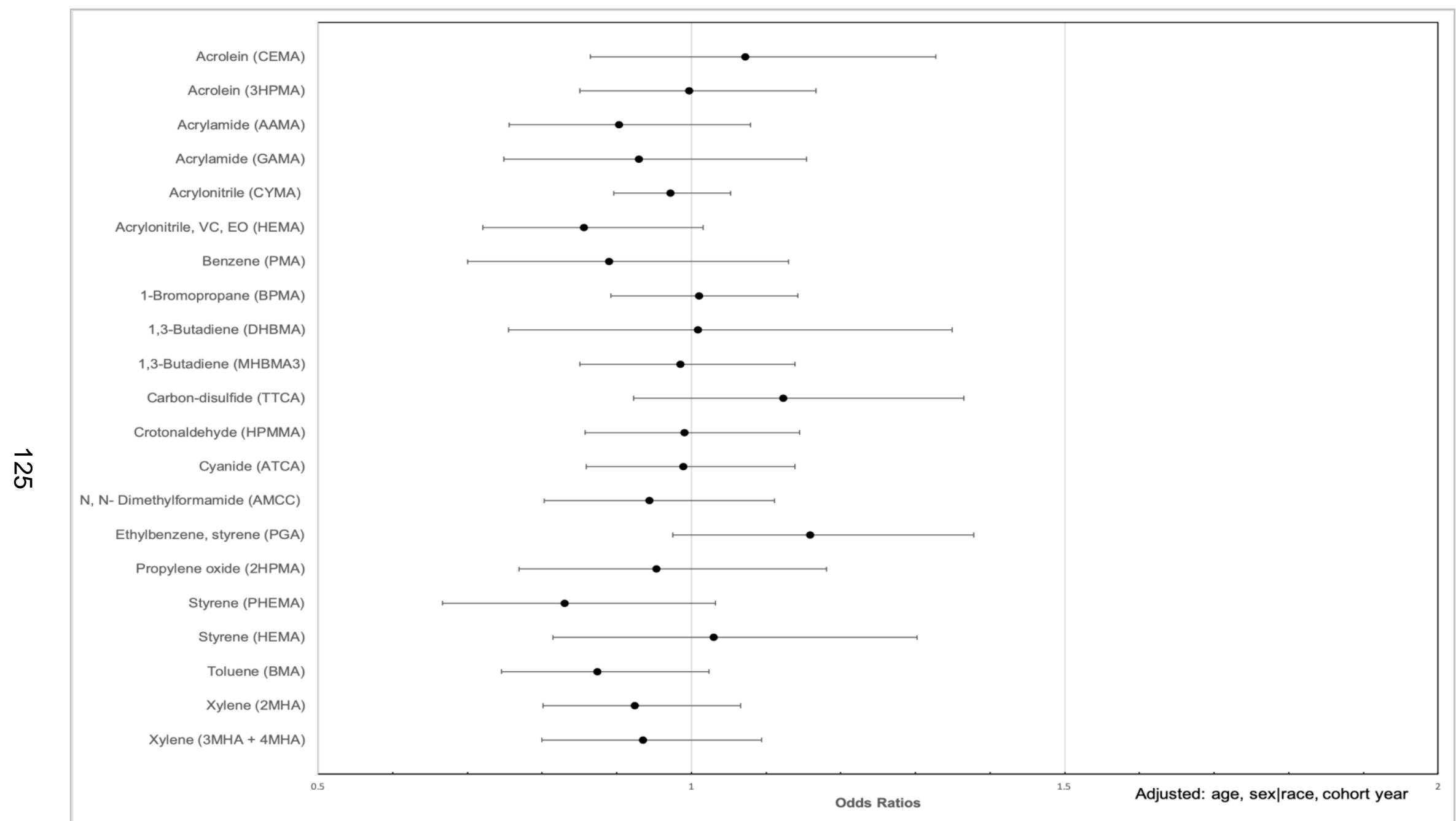

Figure 3.2.6. Forest Plot- Tobacco Smoke Exposed Sub-Population, Adjusted Odds And 95\% CI For 21 UM-VOCs and CMS (Dichotomous)

Note: ${ }^{*}=$ significance at a $p$-value threshold of $<0.05 ; \dagger=$ significant at a $p$-value threshold of $<0.0167$ 


\section{Aim Three}

Dose-response Relationships of UM-VOCs and CMS

Dose-response relationships were assessed by plotting CMS prevalence on the $y$-axis against UM-VOC quantile concentration cut-points on the x-axis. VOC dose was defined using measures urinary metabolites of VOC as a proxy for exposure. UM-VOC quintiles were treated ordinally to assess linear trends, as well as categorically, to examine whether any of the quintiles had significantly higher odds of CMS versus the referent category (lowest exposure quintile).

\section{Unadjusted Trends}

Metabolites of seven VOCs had significant linear trends for the prevalence of CMS at p-value $<0.05$; five UM-VOC (quintiles) were associated with increased odds of CMS (CEMA, DHBMA, HPMMA, ATCA and PGA) (Figure 3.3.1). When the multiple comparisons adjusted significance threshold was applied ( $p$-value $<0.0167$ ), three metabolites had significant linear trends with the prevalence of CMS (CEMA OR: 1.20, 95\%Cl, 1.12-1.29; DHBMA OR, 1.18, 95\%Cl: 1.09-1.28; HPMMA OR, 1.11, 95\%Cl: 1.03-1.19) (Table A8). All three metabolites had an increased odds CMS (Figure 3.3.1). Neither of metabolites inversely associated with CMS prevalence remained significant after multiple comparisons adjustments were applied. 


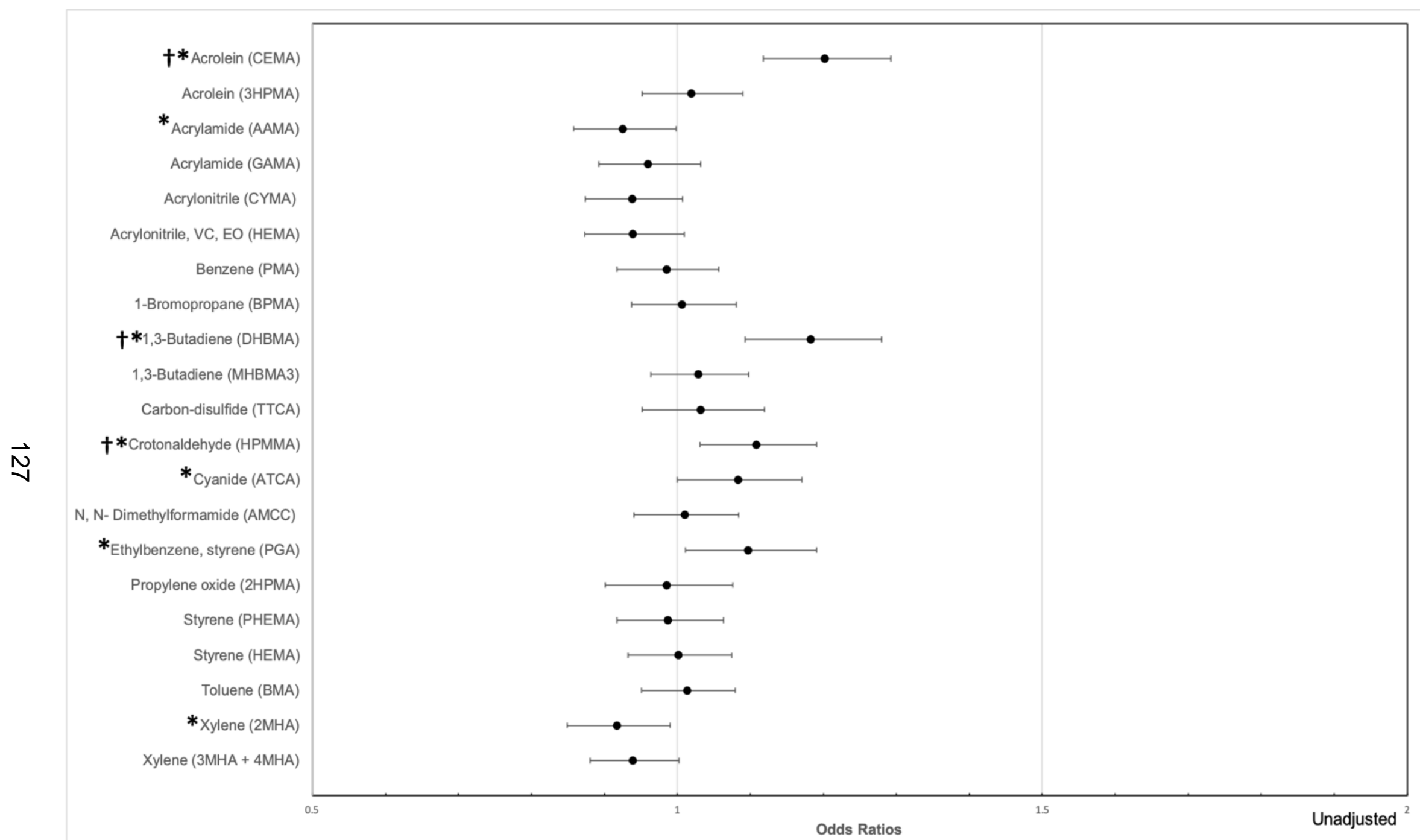

Figure 3.3.1. Forest Plot- Full Cohort, Unadjusted Odds and 95\% Cl for 21 UM-VOCs (quantiles) and CMS (Dichotomous) Note: ${ }^{*}=$ significance at a $p$-value threshold of $<0.05 ; \dagger=$ significant at a $p$-value threshold of $<0.0167$ 
CEMA (acrolein) and DHBMA (1,3-butadiene) had significant doseresponse type relationships- with both significant linear and step-wise categorical relationships with CMS prevalence (Figure 3.3.2). CEMA and DHBMA have significant increases in the prevalence of CMS with each quintile increase in metabolite concentration. HPMMA, a crotonaldehyde metabolite, exhibits a similar pattern with an overall significant linear trend; however, the last quintile of HPMMA concentration falls off with a decrease in prevalence of CMS. ATCA (cyanide) and PGA (ethylbenzene) did not have significant step-wise dose-response relationships but did show a significant ( $p$-value $<0.05$ ) linear trend of increasing CMS prevalence with increasing metabolite concentration. The trends observed with ATCA and PGA did not hold their significance at the p-value $<0.0167$ significance threshold (Table A.8). 

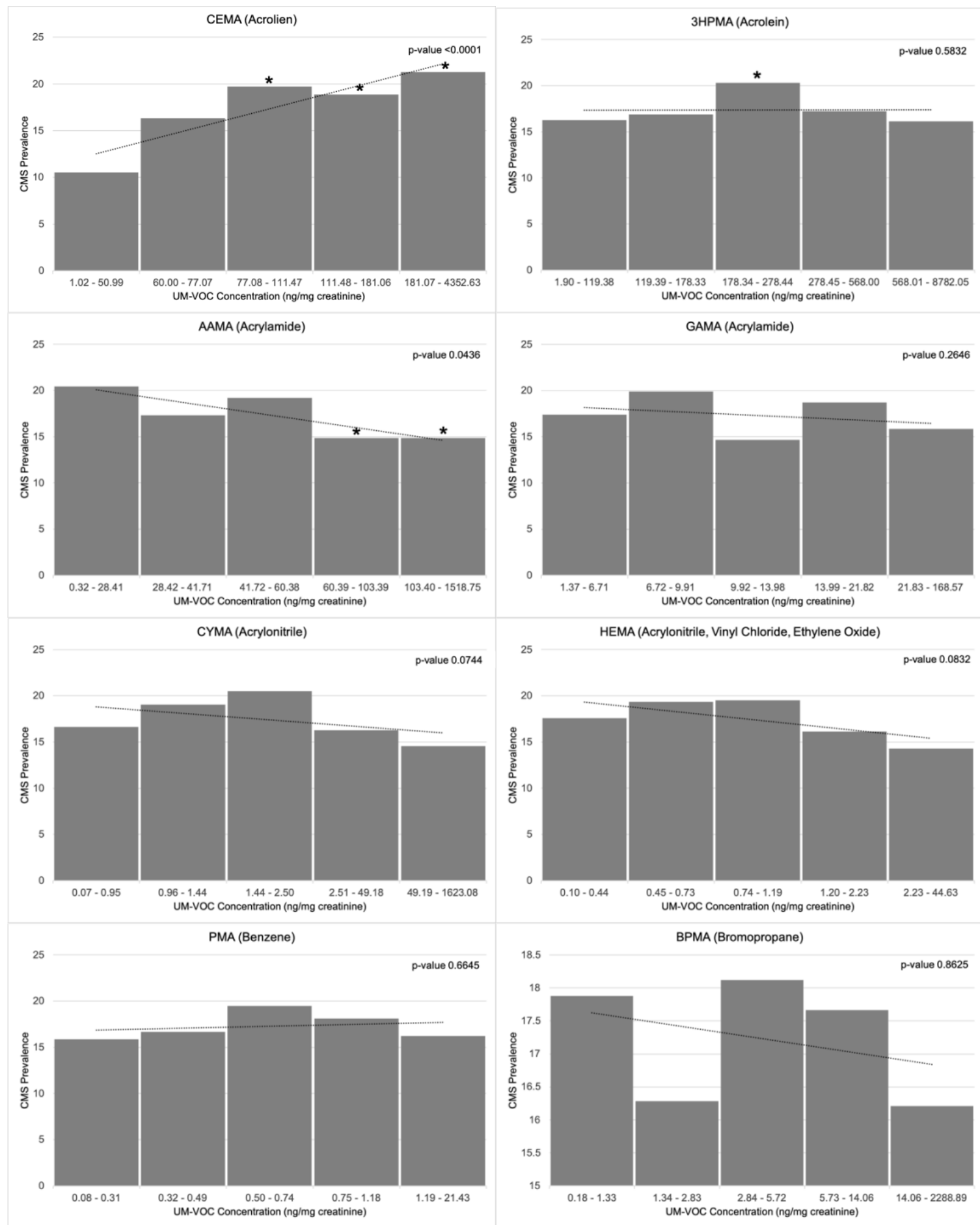


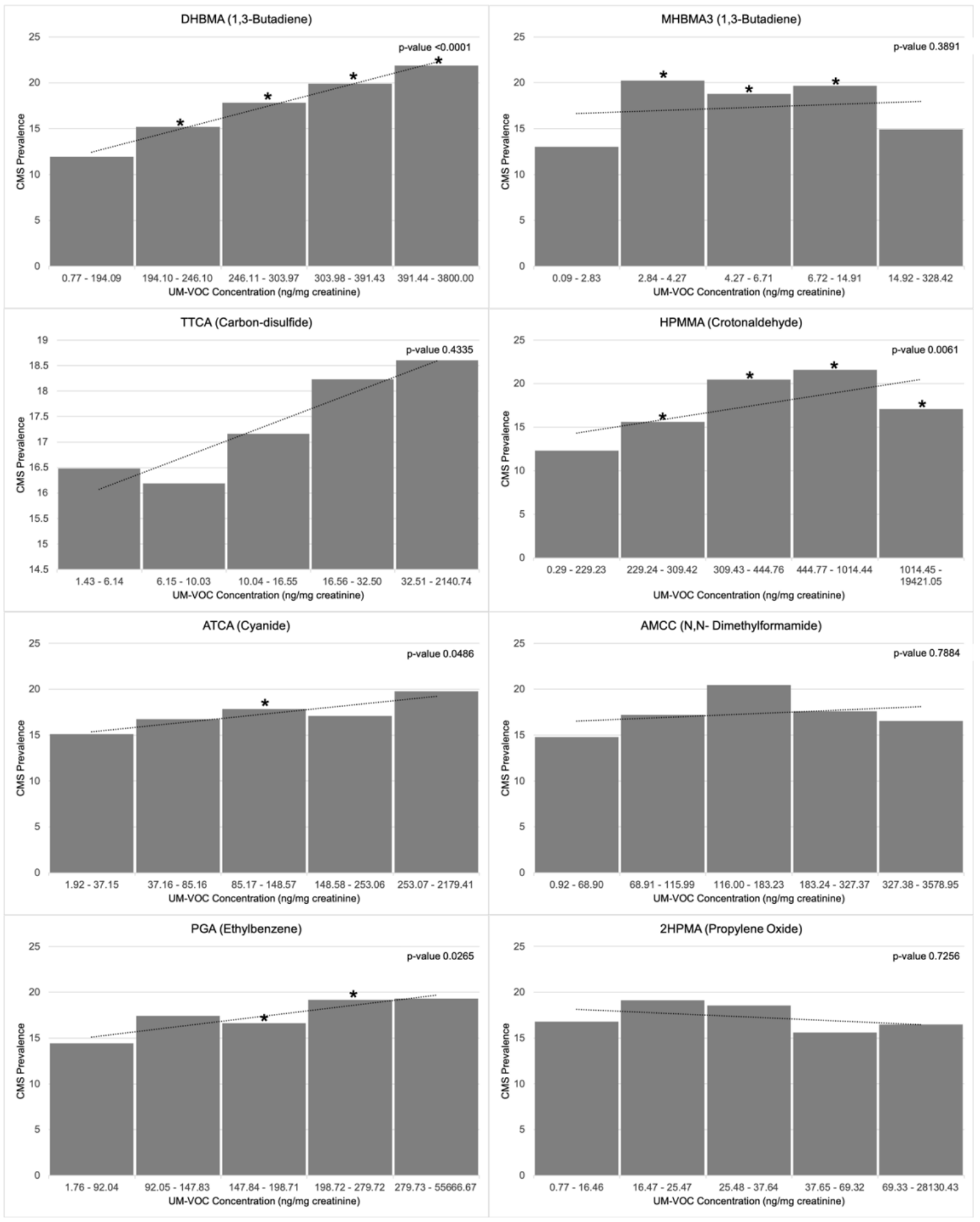



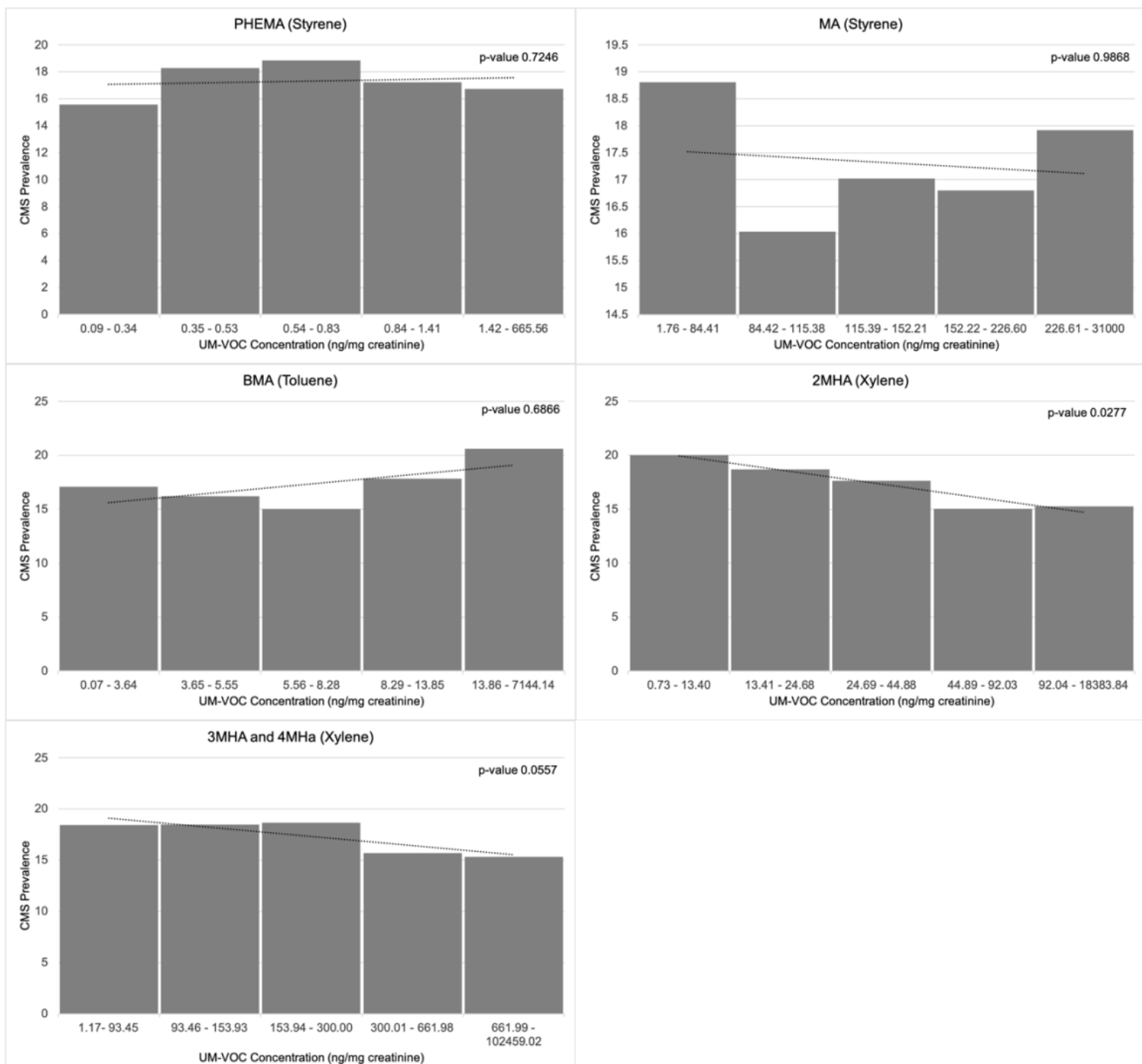

Figure 3.3.2. Full-Cohort, CMS Prevalence by UM-VOC Quintile concentration. Note: $p$-values are the Wald chi-square statistic for linear logistic regression trends; * $=p$-value for significant $(<0.05)$ categorical Wald chi-square comparisons statistic with the referent group being the first quintile of exposure.

\section{Adjusted Trends}

The multivariable logistic regression models adjusted for race, sex, cohort year, and the race and sex interaction showed an increasing trend in the odds of CMS (dichotomous) as CEMA (acrolein) quintiles increased (Figure 3.3.3). Participants had approximately $15 \%$ (CEMA OR: $1.15,95 \% \mathrm{Cl}: 1.07-1.24)$ greater odds of CMS with each ng of CEMA per mg creatinine increase ( $p$-value: 0.0004 ) 
(Table A.9). This relationship was persevered even after the multiple comparisons p-value threshold adjustment. CEMA also displayed significant step-wise increases in CMS with each quintile increase in metabolite concentration. A styrene metabolite, PHEMA, displayed significant decreases in the odds of CMS with increasing metabolite concentrations (PHEMA OR: 0.89, 95\% $\mathrm{Cl}$ : 0.81-0.98) $(p$-value $=0.0146)($ Table A.9). HEMA, PMA, and both xylene metabolites showed significant decreasing trends in the odds of CMS with each quintile increase in metabolite concentration at the $p$-value $<0.05$ threshold but these relationships did not remain significant at the multiple comparisons adjusted significance threshold, p-value $<0.0167$. 


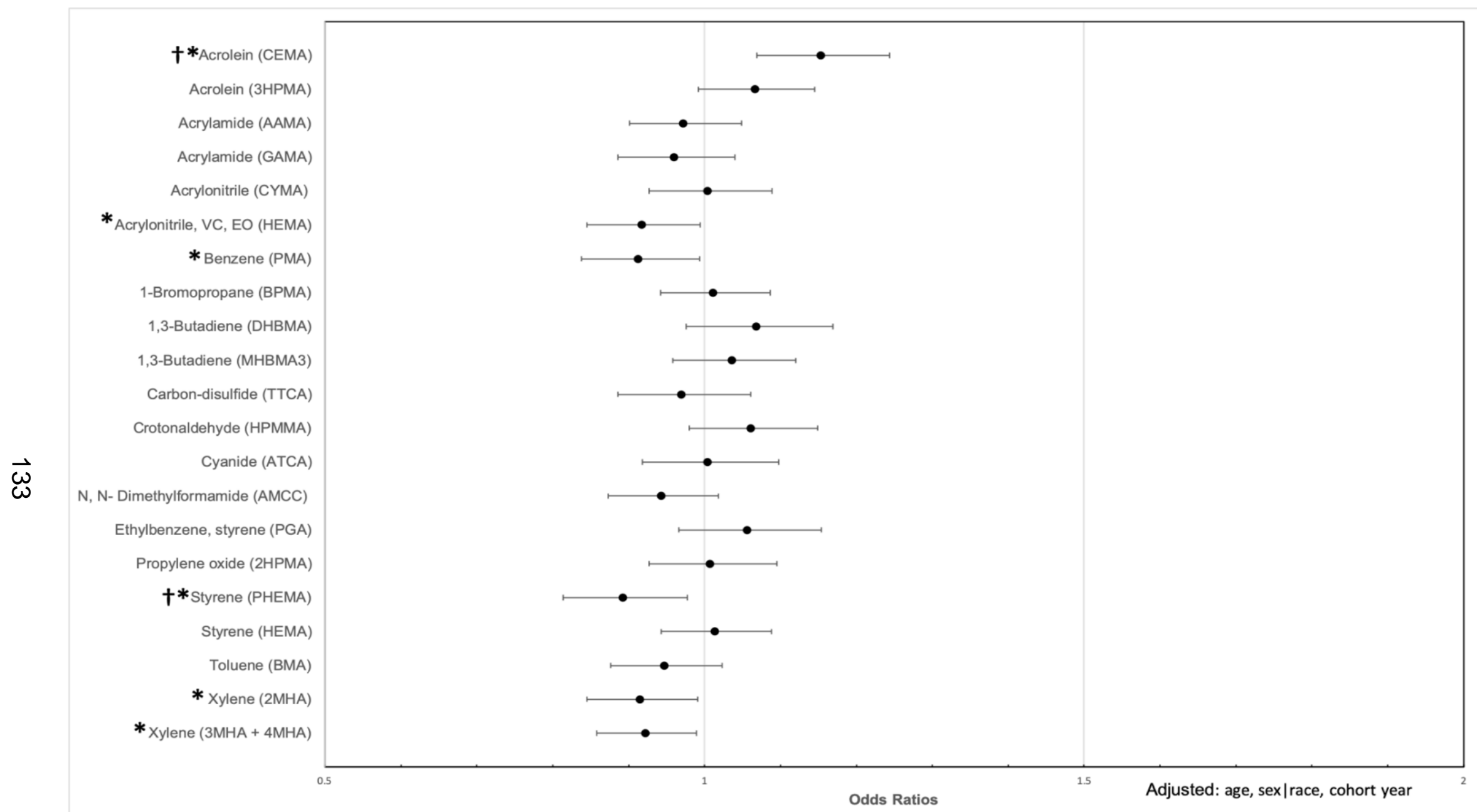

Figure 3.3.3. Forest Plot- Full Cohort, Adjusted Odds and $95 \% \mathrm{Cl}$ for $21 \mathrm{UM}-\mathrm{VOCs}$ (quantiles) and CMS (Dichotomous) Note: Adjusted for age, sex, race and the interaction between sex and race, and NHANES cohort year; odds ratio estimates and the corresponding 95\% Wald confidence intervals $(\mathrm{Cl}) ; p$-value of the Wald chi-square statistic * ${ }^{*}$ significance at a $p$-value threshold of $<0.05 ; \dagger=$ significant at a $p$-value threshold of $<0.0167$. 


\section{Smoking and Non-Smoking Results}

Among the participants in the full appended NHANES cohort who were not exposed to tobacco smoke, two metabolites were significantly associated with CMS at the multiple comparisons adjusted $p$-value threshold. One UM-VOC was significantly positively associated with CMS at the p-value threshold of 0.0167 ; CMEA, a metabolite of acrolein, was associated with increased odds of CMS (CEMA OR: 1.226, 95\% Cl: 0.078-0.391) (Figure 3.3.4). Quintiles of CEMA also maintained a significant step-wise/dose-response type relationship with CMS, as was observed in the unadjusted analysis. 3 and $4 \mathrm{MHA}$ a metabolite of xylene was significantly inversely associated with CMS (3\&4MHA OR: $0.88,95 \% \mathrm{Cl}: 0.79$ 0.97) (Table A.10).

Among the participants in the cohort who were exposed to tobacco smoke, a styrene and a toluene metabolite were inversely associated with CMS at the significance threshold of $p$-value $<0.05$; however, CMS was not significantly associated with any metabolites at the multiple comparisons adjusted significance threshold (p-value 0.0167) (Figure 3.3.5 and Table A.11). 


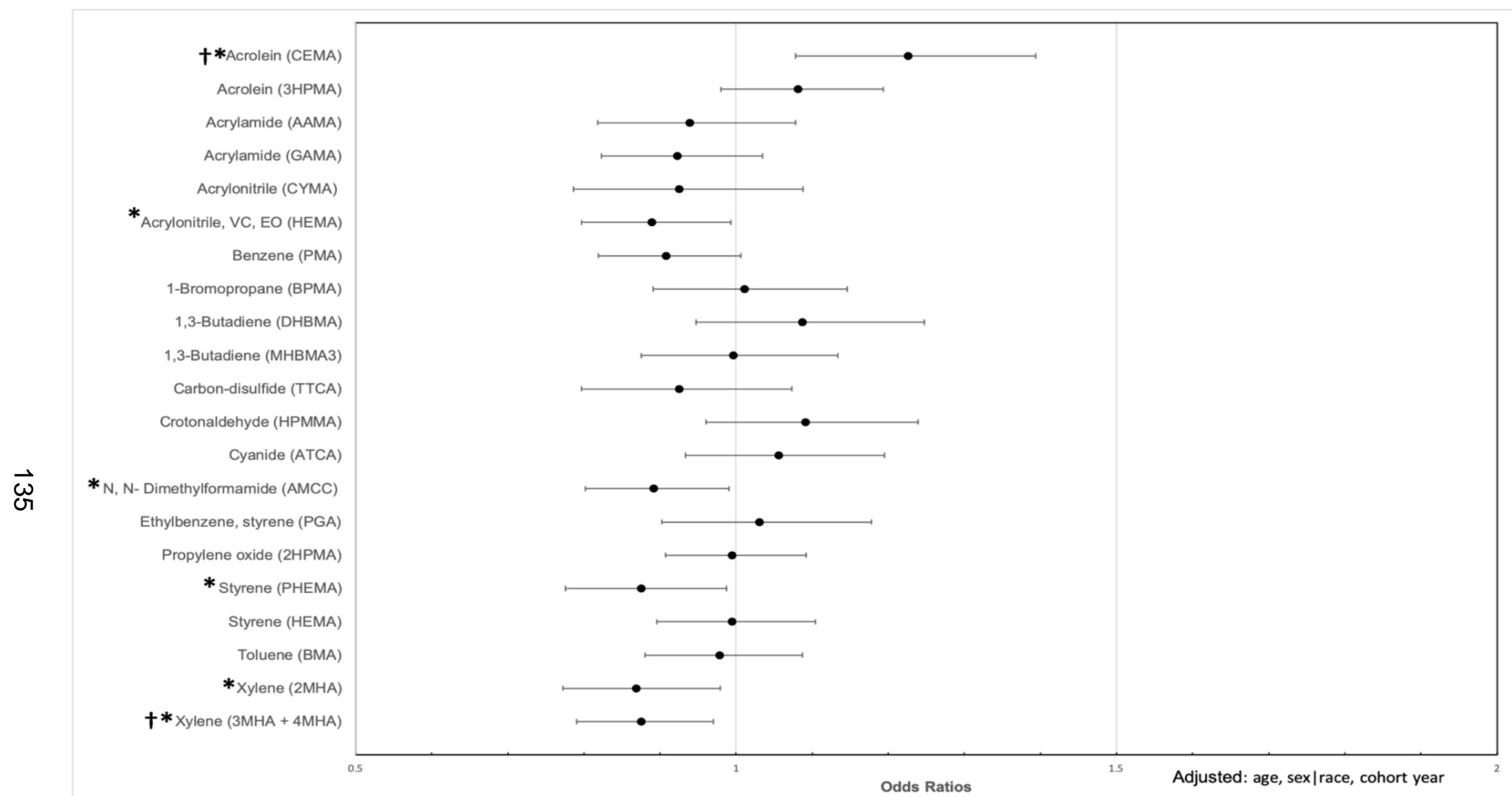

Figure 3.3.4. Forest Plot- Non-Smoke Exposed: Adjusted Odds and 95\% Cl for 21 UM-VOCs (quantiles) and CMS (Dichotomous)

Note: Adjusted for age, sex, race and the interaction between sex and race, and NHANES cohort year; odds ratio estimates and the corresponding $95 \%$ Wald confidence intervals $(\mathrm{Cl}) ; \mathrm{p}$-value of the Wald chi-square statistic * ${ }^{*}$ significance at a $p$-value threshold of $<0.05 ; \dagger=$ significant at a $p$-value threshold of $<0.0167$. 


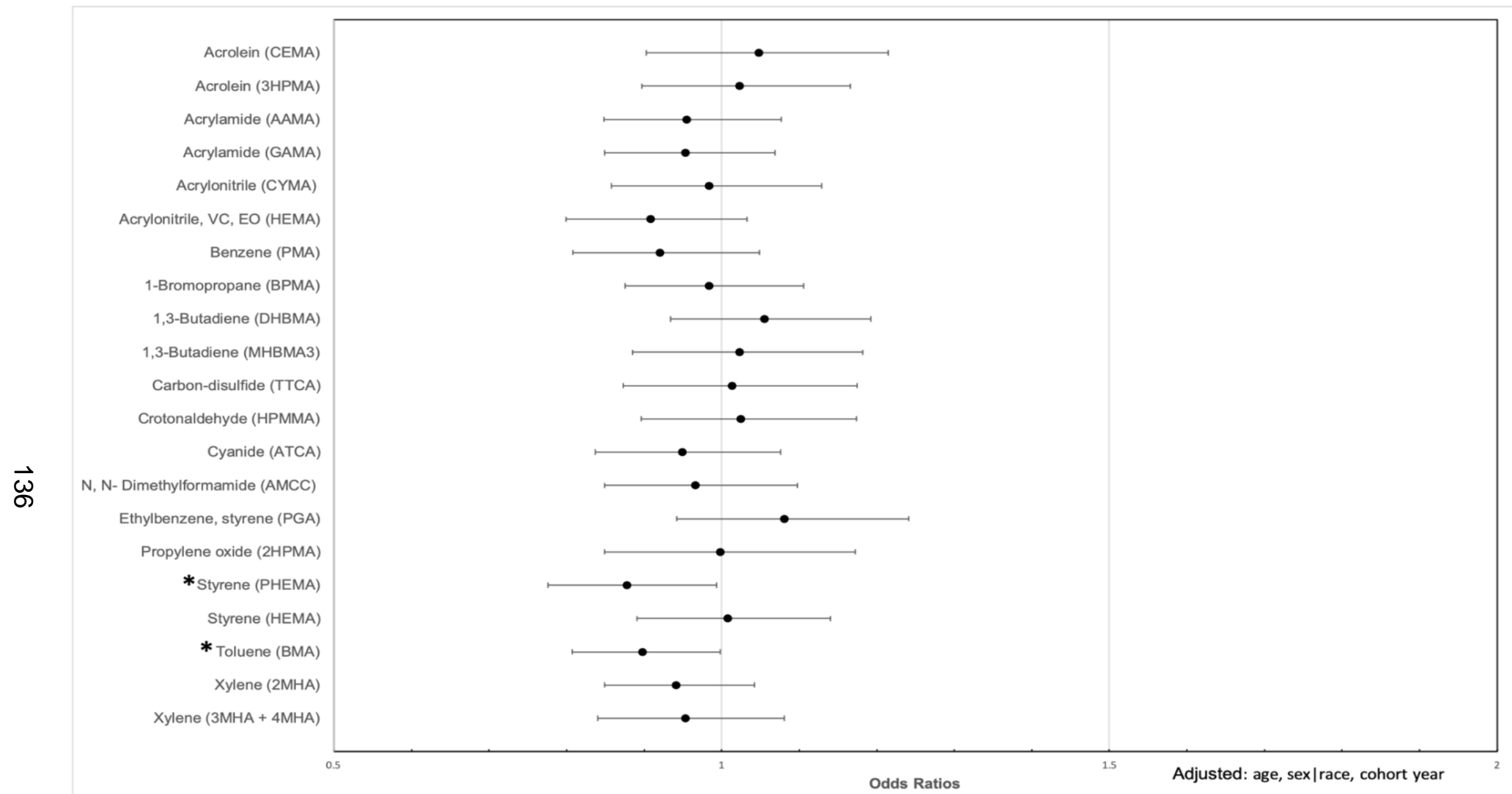

Figure 3.3.5. Forest Plot- Smoke Exposed, Adjusted Odds and 95\% CI for 21 UM-VOCs (quantiles) and CMS (Dichotomous)

Note: Adjusted for age, sex, race and the interaction between sex and race, and NHANES cohort year; odds ratio estimates and the corresponding $95 \%$ Wald confidence intervals $(\mathrm{Cl}) ; p$-value of the Wald chi-square statistic * $=$ significance at a $p$-value threshold of $<0.05 ; \dagger=$ significant at a $p$-value threshold of $<0.0167$. 


\section{Aim Four}

The combinatory effects of VOCs associated with CMS.

\section{Ordinal Logistic Regression}

UM-VOCs were first individually assessed for associations with the ordinal CMS-risk score (CMS-RS). The CMS-RS is an ordinal variable designed to organize individuals into ordinal categories based on the number of CMS risk factors an individual has (0-7) (Table 2.3). Due to low numbers in categories 4 and 7 of the proposed ordinal categories for the CMS-RS, categories 3 and 4 and categories 6 and 7 were condensed into 1 category each; making 6 categories (CMS-RS 0-5) instead of 8 . The Score Chi-Square test for proportional odds assumption was not significant with respect to the chi-square distribution, which indicates that the proportional odds model adequately fit the data for all VOC's.

The unadjusted ordinal survey-weighted logistic regression models identified 8 UM-VOCs significant at the p-value $<0.05$ threshold, with six metabolites positively associated with the ordinal CMS risk score (Figure 3.4.1) After applying the multiple comparisons adjusted $p$-value threshold, ( $p$-value $<0.0167)$ four UM-VOCs remained significantly positively associated with the ordinal CMS-RS (CEMA OR: 1.38, 95\%Cl: 1.27-1.51; DHBMA OR: 1.34, 95\%Cl: 1.14-1.58; AMCC OR: 1.12, 95\%Cl: 1.03-1.21; PGA OR: 1.18, 95\%Cl: 1.11-1.25;) (Table A.12). CYMA (OR: $1.20,95 \% \mathrm{Cl}: 1.12-1.29)$ was significantly negatively associated with CMS-RS (Table A.12).

Ordinal logistic regression models adjusted for age, sex, race, the 
interaction between sex and race, and NHANES cohort year were significant for six metabolites (Figure 3.4.2); two of these metabolites were positively associated with the CMS-RS (CEMA OR: 1.27, 95\%Cl: 1.16-1.39; PGA OR: 1.17, 95\%Cl: 1.09-1.25), and four were negatively associated (HEMA OR: $0.89,95 \% \mathrm{Cl}: 0.82-$ 0.98; PMA OR: $0.80,95 \% \mathrm{Cl}$ : 0.71-0.89; TTCA OR: 0.90, 95\%Cl: $0.84-0.96$; PHEMA OR: 0.84, 95\%Cl: 0.76-0.93) (Table A.13). 


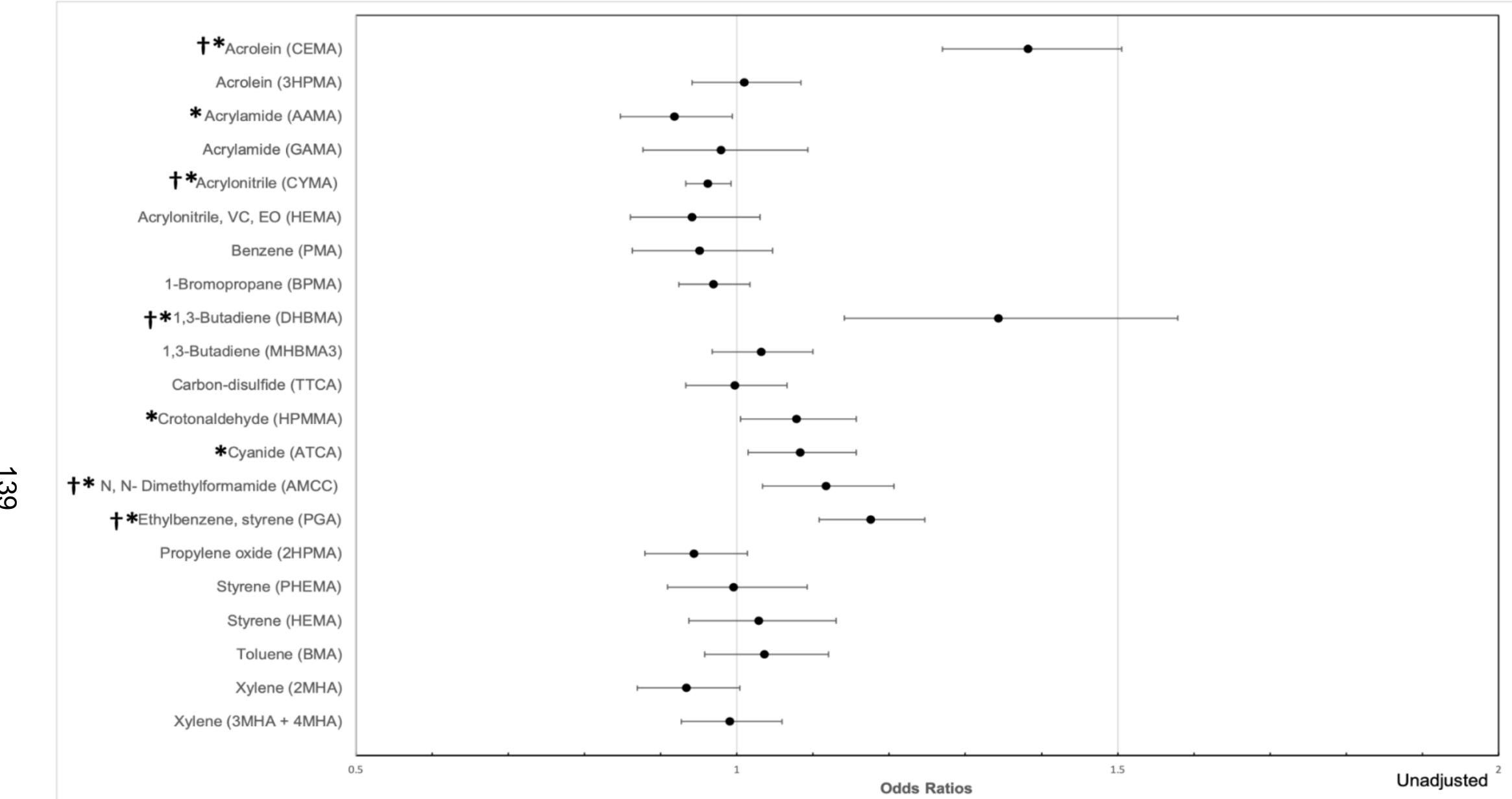

Figure 3.4.1. Forest Plot- Full cohort, Unadjusted ordinal odds and 95\% Cl for $21 \mathrm{UM}$-VOCs and cardiometabolic syndrome risk score (CMS-RS).

Note: ${ }^{*}=$ significance at a $p$-value threshold of $<0.05 ; \dagger=$ significant at a $p$-value threshold of $<0.0167$. 


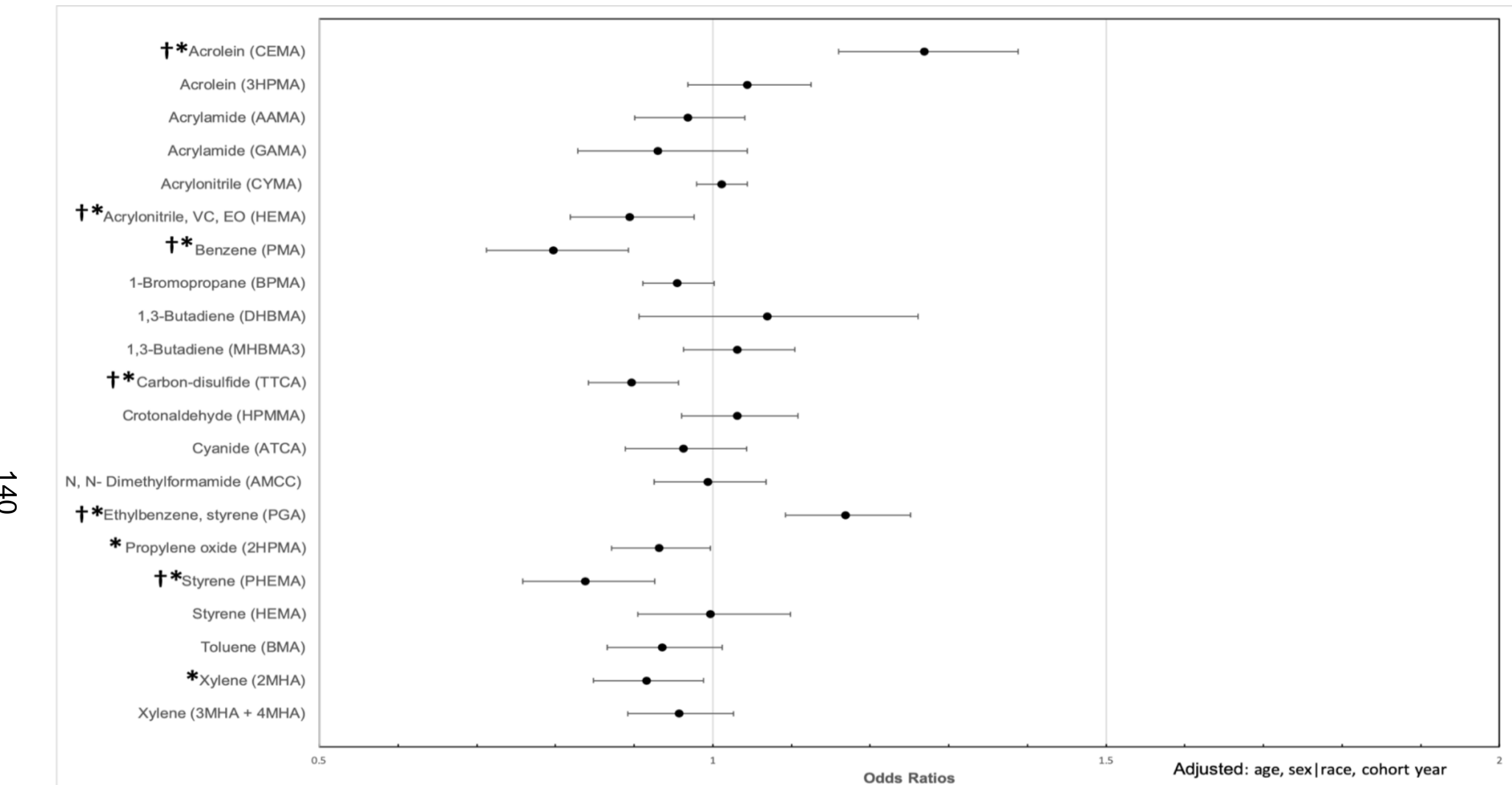

Figure 3.4.2. Forest Plot- Full cohort, Adjusted ordinal odds and 95\% Cl for 21 UM-VOCs and cardiometabolic syndrome risk score (CMS-RS).

Note: Adjusted for age, sex, race and the interaction between sex and race, and NHANES cohort year; odds ratio estimates and the corresponding 95\% Wald confidence intervals $(\mathrm{Cl}) ; \mathrm{p}$-value of the Wald chi-square statistic * ${ }^{*}$ significance at a $p$-value threshold of $<0.05 ; \dagger=$ significant at a $p$-value threshold of $<0.0167$. 
The models were then stratified by tobacco smoke exposure. Among the tobacco smoke exposed subpopulation, only one metabolite, PGA (a metabolite of ethylbenzene/styrene) was significantly positively associated ( $p$-value threshold $<0.0167$ ) with the CMS-RS (PGA OR: 1.17, 95\%Cl: 1.05-1.31) (Figure 3.4.3, Table A.14). Among the non-tobacco smoke exposed cohort, one metabolite, CEMA (a metabolite of acrolein) was significantly positively associated $(p<0.0167)$ with the CMS-RS (CEMA OR: 1.39, 95\%Cl: 1.19-1.62) (Figure 3.4.4, Table A.15). Several of the VOC's were significantly inversely associated with the CMS-RS in both the smoke and non-smoke exposed subpopulations. 


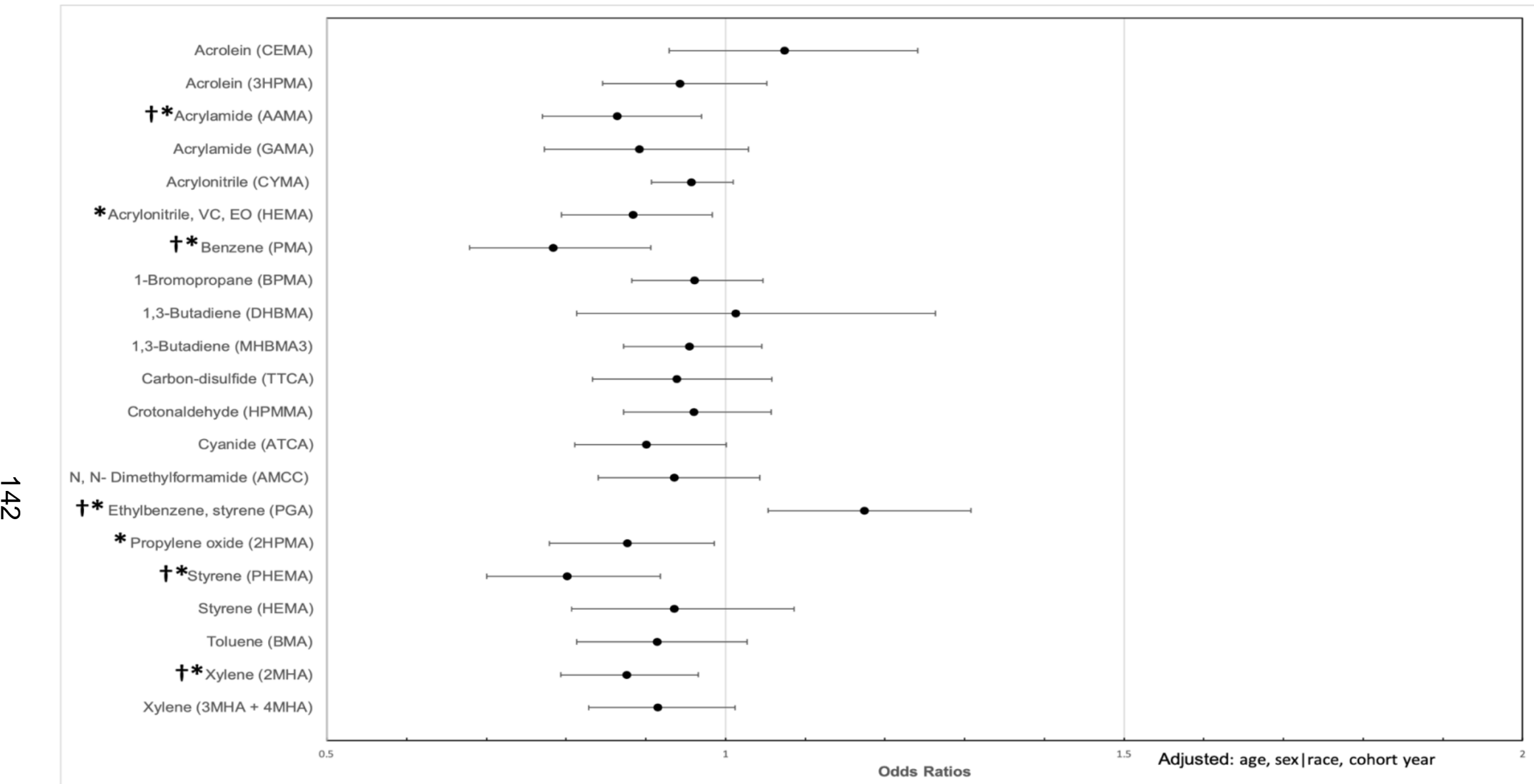

Figure 3.4.3. Forest Plot- Tobacco Smoke Exposed, Adjusted ordinal odds and 95\% Cl for 21 UM-VOCs and cardiometabolic syndrome risk score (CMS-RS).

Note: Adjusted for age, sex, race and the interaction between sex and race, and NHANES cohort year; odds ratio estimates and the corresponding $95 \%$ Wald confidence intervals $(\mathrm{Cl}) ; \mathrm{p}$-value of the Wald chi-square statistic * ${ }^{*}$ significance at a $p$-value threshold of $<0.05 ; \dagger=$ significant at a $p$-value threshold of $<0.0167$. 


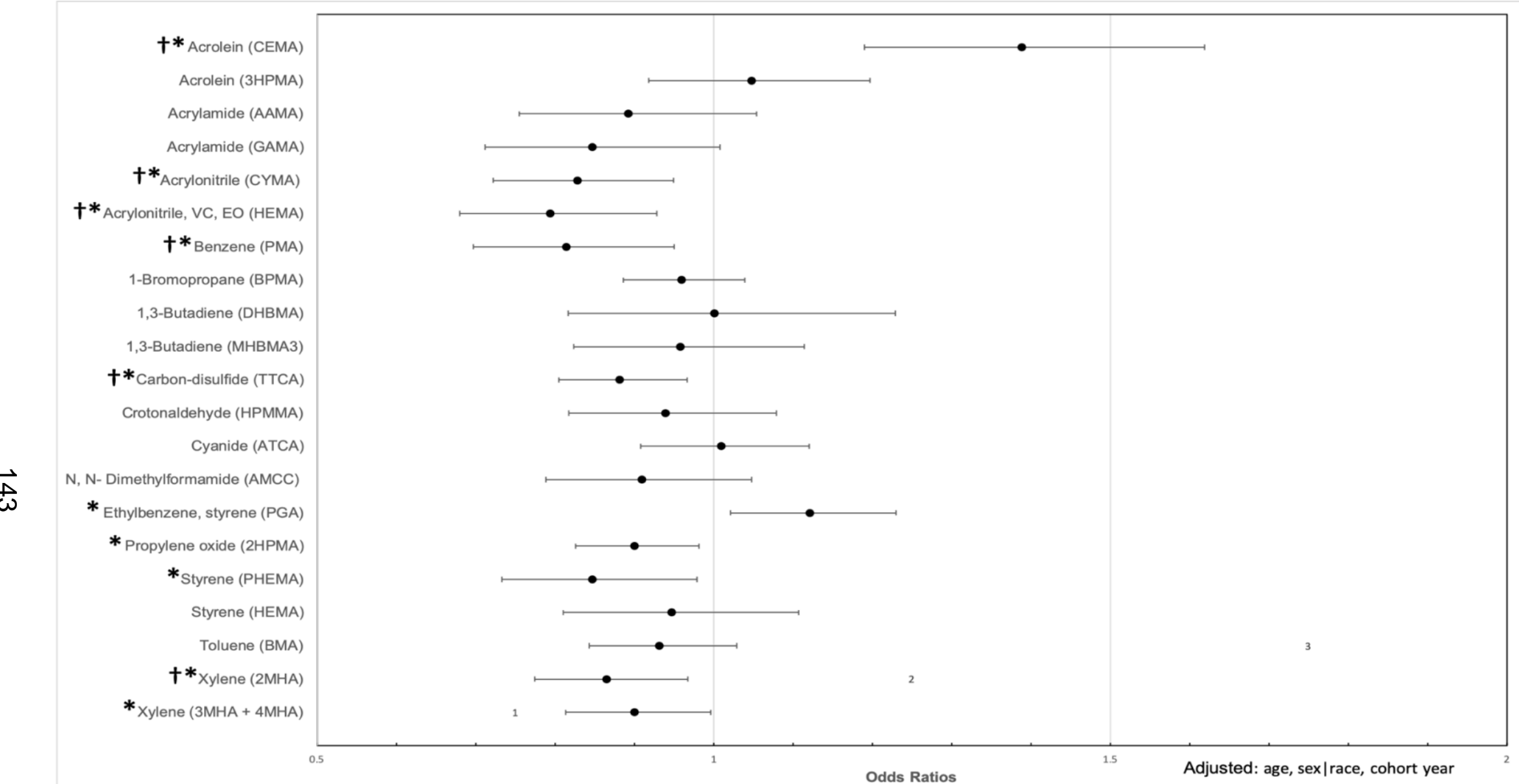

Figure 3.4.4. Forest Plot- Non-tobacco Smoke Exposed, Adjusted ordinal odds and 95\% Cl for 21 UM-VOCs and cardiometabolic syndrome risk score (CMS-RS).

Note: Adjusted for age, sex, race and the interaction between sex and race, and NHANES cohort year; odds ratio estimates and the corresponding 95\% Wald confidence intervals $(\mathrm{Cl}) ; \mathrm{p}$-value of the Wald chi-square statistic * ${ }^{*}$ significance at a $p$-value threshold of $<0.05 ; \dagger=$ significant at a $p$-value threshold of $<0.0167$. 


\section{Backwards Selection}

Two-way interactions were assessed to address if the effect of a given metabolite is dependent on the concentrations of another metabolite, ultimately affecting the response of CMS. UM-VOCs selected for inclusion in the backward elimination analysis to identify potentially significant 2-way interactions are identified in Table 3.4.1. Thirteen representative UM-VOCs were included in the logistic regression backward elimination analysis, including one representative

metabolite for each parent VOC. Representative metabolites were selected only from metabolites with greater than $40 \%$ of samples measured above LOD. If a parent VOC had multiple metabolites, the metabolite with the greatest significance in the fully adjusted ordinal logistic regression models was selected. If a VOC had multiple metabolites but none with significant associations with CMS in the fully adjusted ordinal logistic regression models, the metabolite with the greatest abundance was selected as the representative metabolite for that VOC.

The backward elimination analysis utilized PROC LOGISTIC. Ninety-two effects were entered into the model; 10 individual UM-VOCs and 6 two-way interactions between UM-VOCs were retained in the model using a significance level of 0.05 . The 10 individual UM-VOCs retained in the model were; CEMA, AAMA, CYMA, BPMA, DHBMA, HPMMA, ATCA, PGA, MA, and 2MHA. The six two-way interactions that were retained in the model were: AAMA and BPMA, CYMA and BPMA, AAMA and HPMMA, ATCA and MA, CEMA and 2MHA, and DHBMA and 2MHA.

To assess how the response to CMS depends on the interactions identified 
from the backward selection analysis, each of the six identified interaction terms and each of the 10 individual UM-VOCs, were then individually placed into its own survey weighted logistic regression model adjusted for age, sex, race, the interaction between sex and race, and NHANES cohort year.

After assessing the significance ( $p$-value $<0.05$ ) of the interaction terms and the individual UM-VOCs in their own models, the final model identified from the backwards selection logistic regression technique with CMS (yes/no) as the outcome variable included the individual UM-VOCs CEMA, PGA, and 2MHA as well as an interaction term and lower order components for CYMA and BPMA; adjustments for age, sex, race, the interaction between sex and race, and NHANES cohort year were also included in the model. 
Table 3.4.1. Identification of UM-VOCs included in backward selection analysis.

\begin{tabular}{|c|c|c|c|c|c|c|c|c|}
\hline \multirow[b]{2}{*}{ Parent Compound } & \multirow[b]{2}{*}{ Metabolite } & \multirow[b]{2}{*}{$\begin{array}{l}\text { Considered } \\
\text { for 2-way } \\
\text { Interactions }\end{array}$} & \multicolumn{3}{|c|}{$\begin{array}{c}\text { Significance in Ordinal } \\
\text { Logistic Regrassion } \\
\text { Models }\end{array}$} & \multicolumn{3}{|c|}{ Percent Above LOD } \\
\hline & & & $\begin{array}{c}\text { Full } \\
\text { Cohort }\end{array}$ & $\begin{array}{l}\text { Smoke } \\
\text { Exposed }\end{array}$ & $\begin{array}{c}\text { Non } \\
\text { Smoke } \\
\text { Exposed }\end{array}$ & $\begin{array}{c}\text { Appended } \\
n=4654\end{array}$ & $\begin{array}{l}\text { Smoke } \\
\text { Exposed } \\
n=2176\end{array}$ & $\begin{array}{c}\text { Non } \\
\text { Smoke } \\
\text { Exposed } \\
n=2110\end{array}$ \\
\hline \multirow[t]{2}{*}{ Acrolein } & CEMA & $\#$ & $t^{*}$ & & $t^{*}$ & 98.62 & 99.27 & 97.49 \\
\hline & 3HPMA & & & & & 99.41 & 99.56 & 99.06 \\
\hline \multirow[t]{2}{*}{ Acrylamide } & AAMA & $\#$ & & $t^{*}$ & & 99.49 & 99.68 & 99.38 \\
\hline & GAMA & & & & & 47.93 & 60.66 & $37.73^{\wedge}$ \\
\hline Acrylonitrile & CYMA & $\#$ & & & $\dagger^{*}$ & 90.09 & 95.29 & 82.32 \\
\hline $\begin{array}{l}\text { Acrylonitrile, vinyl } \\
\text { chloride, ethylene oxide }\end{array}$ & HEMA & & $\dagger^{*}$ & * & $\dagger^{*}$ & 40.63 & 50.59 & $26.87^{\wedge}$ \\
\hline Benzene & PMA & & $\dagger^{*}$ & $\dagger^{*}$ & $\dagger^{*}$ & $32.42^{\wedge}$ & $35.99^{\wedge}$ & $29.45^{\wedge}$ \\
\hline 1-Bromopropane & BPMA & \# & & & & 73.02 & 72.03 & 71.50 \\
\hline \multirow[t]{2}{*}{ 1,3-Butadiene } & DHBMA & $\#$ & & & & 99.76 & 99.64 & 99.79 \\
\hline & МНBMAЗ & & & & & 97.51 & 97.87 & 96.37 \\
\hline Carbon-disulfide & TTCA & & $\dagger^{*}$ & & $\dagger^{*}$ & $38.38^{\wedge}$ & $39.99^{\wedge}$ & $33.82^{\wedge}$ \\
\hline Crotonaldehyde & HPMMA & $\#$ & & & & 99.93 & 99.94 & 99.99 \\
\hline Cyanide & ATCA & $\#$ & & & & 85.45 & 82.58 & 84.85 \\
\hline \multicolumn{9}{|l|}{ Dimethylformamide } \\
\hline Ethylbenzene, styrene & PGA & $\#$ & $\dagger^{*}$ & $\dagger^{*}$ & * & 92.99 & 92.48 & 91.11 \\
\hline Propylene oxide & 2HPMA & $\#$ & * & * & * & 93.89 & 96.52 & 91.04 \\
\hline \multirow[t]{2}{*}{ Styrene } & PHEMA & & $\dagger^{*}$ & $\dagger^{*}$ & * & $24.97^{\wedge}$ & $27.94^{\wedge}$ & $16.87^{\wedge}$ \\
\hline & MA & $\#$ & & & & 98.52 & 98.97 & 98.29 \\
\hline Toluene & BMA & $\#$ & & & & 99.12 & 98.61 & 99.09 \\
\hline \multirow[t]{2}{*}{ Xylene } & $2 \mathrm{MHA}$ & $\#$ & $\dagger^{*}$ & $t^{*}$ & $\dagger^{*}$ & 93.82 & 96.84 & 91.47 \\
\hline & $3 \& 4 \mathrm{MHA}$ & & & & * & 99.54 & 99.84 & 99.39 \\
\hline
\end{tabular}

Note: UM-VOC significance in ordinal logistic regression models and percent above LOD for each metabolite for the full cohort, tobacco smoke exposed individuals, and non-tobacco smoke exposed individuals. ${ }^{\wedge}=<40 \%$ of samples above LOD. ${ }^{*}=$ Wald chi-square statistic, significance at a $p$-value threshold of $<0.05$; $\dagger=$ significant at a $p$-value threshold of $<0.0167$. $\#=$ metabolite selected for each parent VOC to be tested for 2-way interactions.

In the final full model built using the backward selection technique, the betas for CYMA and BPMA were not significant. However, the betas for CEMA, PGA, 2MHA, and the interaction term CYMA*BPMA were significant. The beta for the interaction of CYMA and BPMA was negative, indicating that the combined effect of both VOC's is slightly less than would be expected if they were behaving completely independently (Table 3.4.2). The beta for 2MHA was also negative. 
Table 3.4.2. Analysis of maximum likelihood estimates for the final logistic regression model produced by the backwards selection method

\begin{tabular}{lccc}
\hline & \multicolumn{3}{c}{ Standard } \\
Parameter & Estimate & Error & $\operatorname{Pr}>|\mathrm{t}|$ \\
\hline CYMA & 0.171 & 0.197 & 0.3904 \\
BPMA & 0.055 & 0.0654 & 0.4089 \\
CYMA ${ }^{*}$ BPMA & -0.198 & 0.0776 & 0.0143 \\
CEMA & 0.326 & 0.1604 & 0.0476 \\
PGA & 0.253 & 0.0962 & 0.0114 \\
MHA2 & -0.195 & 0.0693 & 0.0072 \\
\hline
\end{tabular}

\section{Factor Analysis}

Three latent variables were identified from the factor analysis conducted in Aim 2. To help reduce collinearity, the factor analysis was conducted using only the non-tobacco smoke exposed population of the cohort and only with UM-VOCs with greater than $40 \%$ of samples measured above LOD. The rotated factor pattern analysis and accompanying path diagram (Figure 3.4.5) show directed links from factors to variables that are associated with a 0.3 or greater magnitude in loading estimates. The key metabolites found in factor 1 were toluene, N,Ndimethylformamide, 1,3-butadiene, acrylamide, ethylbenzene/styrene, and styrene, which explained $56 \%$ of the variance in the data. Factor 2, which explained $36 \%$ of the variance in the data, was distinguished by propylene oxide, 1-brompropane, and acrolein. Factors 1 and 2 share crotonaldehyde, 1,3butadiene, and acrolein. Factor 3 explained $8 \%$ of the variance in the data. The metabolites extracted by factor 3 were both of the xylene metabolites.

In an ordinal logistic regression analysis of the identified 3 latent factors and the CMS-RS, factors 1 and 2 were not significant in the model after adjusting for 
age, sex, race, the interaction between sex and race, and NHANES cohort year (factor 1 OR: 1.11, 95\%Cl: 0.97-1.26; factor 2 OR: 0.96, 95\%Cl: 0.85-1.08). Factor 3 was significantly associated with a decreased odds of ordinal CMS risk. For every unit increase in of factor 3 there was a significant $0.79(95 \% \mathrm{Cl}: 0.72-0.87)$ times lower odds in ordinal CMS risk. The proportional odds assumption was met for this model. 


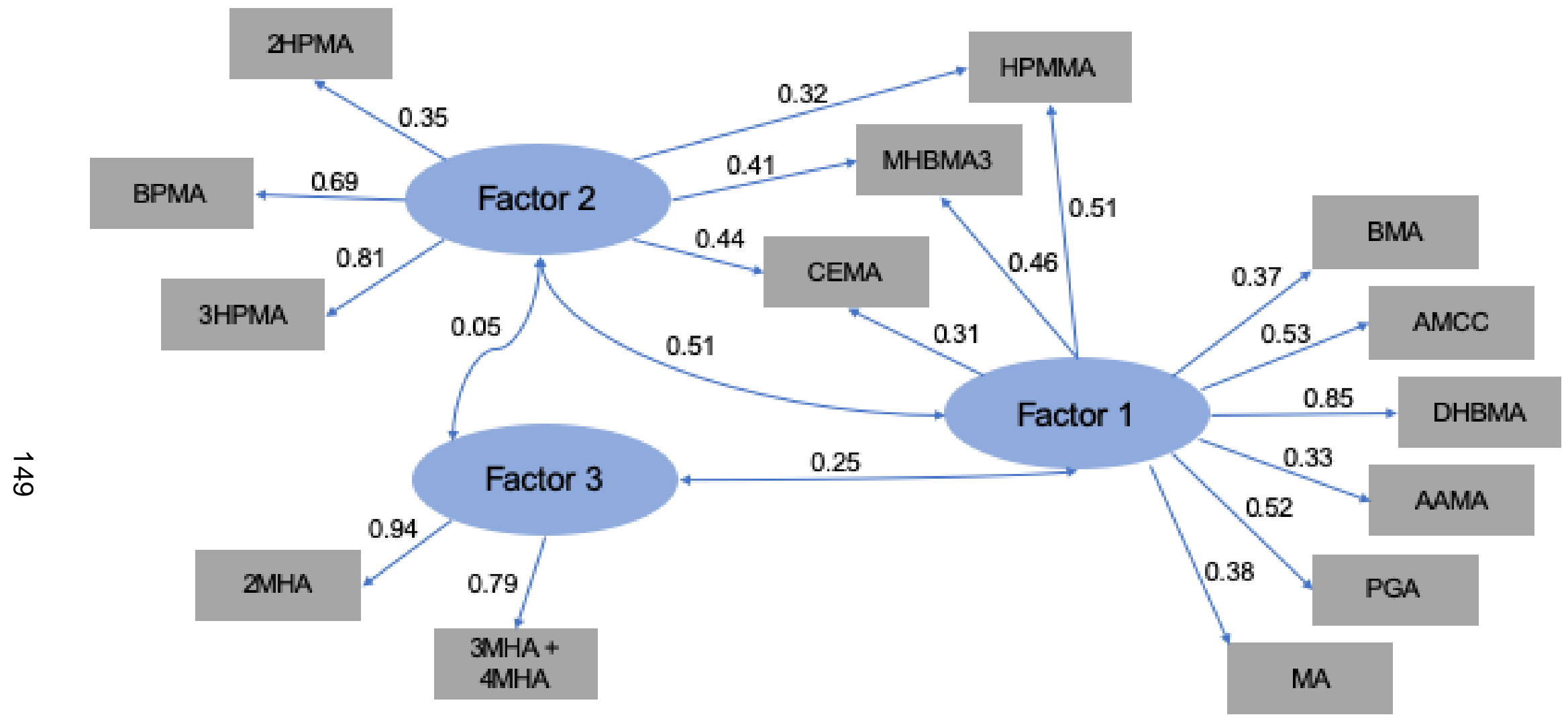

Figure 3.4.5. Factor analysis path diagram of NHANES urinary metabolites of volatile organic compounds.

Note: Creatinine-normalized log-transformed UM-VOCs were used in the factor analysis. Loading estimates $>0.3$ are indicated by the directed links from factors to variables. The double-headed links show correlations between factors.

NHANES= National Health and Nutrition Examination Survey; UM-VOC= urinary metabolite of volatile organic compound. 


\section{Least Absolute Shrinkage and Selection Operator (LASSO) Method}

A generalized linear model employing the LASSO fit procedure was used to identify which of the $21 \mathrm{UM}$-VOCs best predicts prevalent CMS. The large sample size of the cohort allowed for the optimum model to be selected by estimating the prediction error of the models directly via the use of a validation data set to tune the penalized regression. The training observations set $(n=2886)$ was used to produce the solution path while the validation set $(n=1186)$ was used to estimate the prediction error of each of the models on the solution path.

Figure 3.4.6 shows the ASE of the models on the LASSO solution path separately for the training and validation sets. The LASSO solution path is created on a grid of the tuning parameter value $(\mathrm{t})$. Along the $\mathrm{X}$ axis is the normalized tuning values (the $L_{1}$ norm of the regression coefficients divided by the $L_{1}$ norm of the ordinary least squares solution). Model 18 is the model with the smallest ASE produced by the validation data and is therefore the best fit model. Moving from left to right across the $X$ axis, the model complexity increases; i.e., the amount of shrinkage that is imposed on the regression coefficients deceases. For the training data, the model complexity increases and the ASE decreases; but for the validation data, the prediction error decreases and then increases as the model gets more complex. The vertical line in the errors plots indicate the point of minimum ASE. This point indicates that the variables before this line of distinction have important effects in explaining the variation in CMS. The variables after this line describe random noise in the training data that is not reproducibly observed in the validation data. 
The variables with explanatory value chosen by the validation criteria in a generalized linear model employing the LASSO fit procedure for all 21 UM-VOCs in the full study cohort can be observed in the coefficient progression plot (Figure 3.4.7). The UM-VOCs with explanatory power in a linear model predicting prevalent CMS are: CEMA, 3HPMA, AAMA, GAMA, CYMA, HEMA, PMA, BPMA, DHBMA, TTCA, HPMMA, ATCA, AMCC, PGA, 2HPMA, PHEMA, MA, and 2MHA.

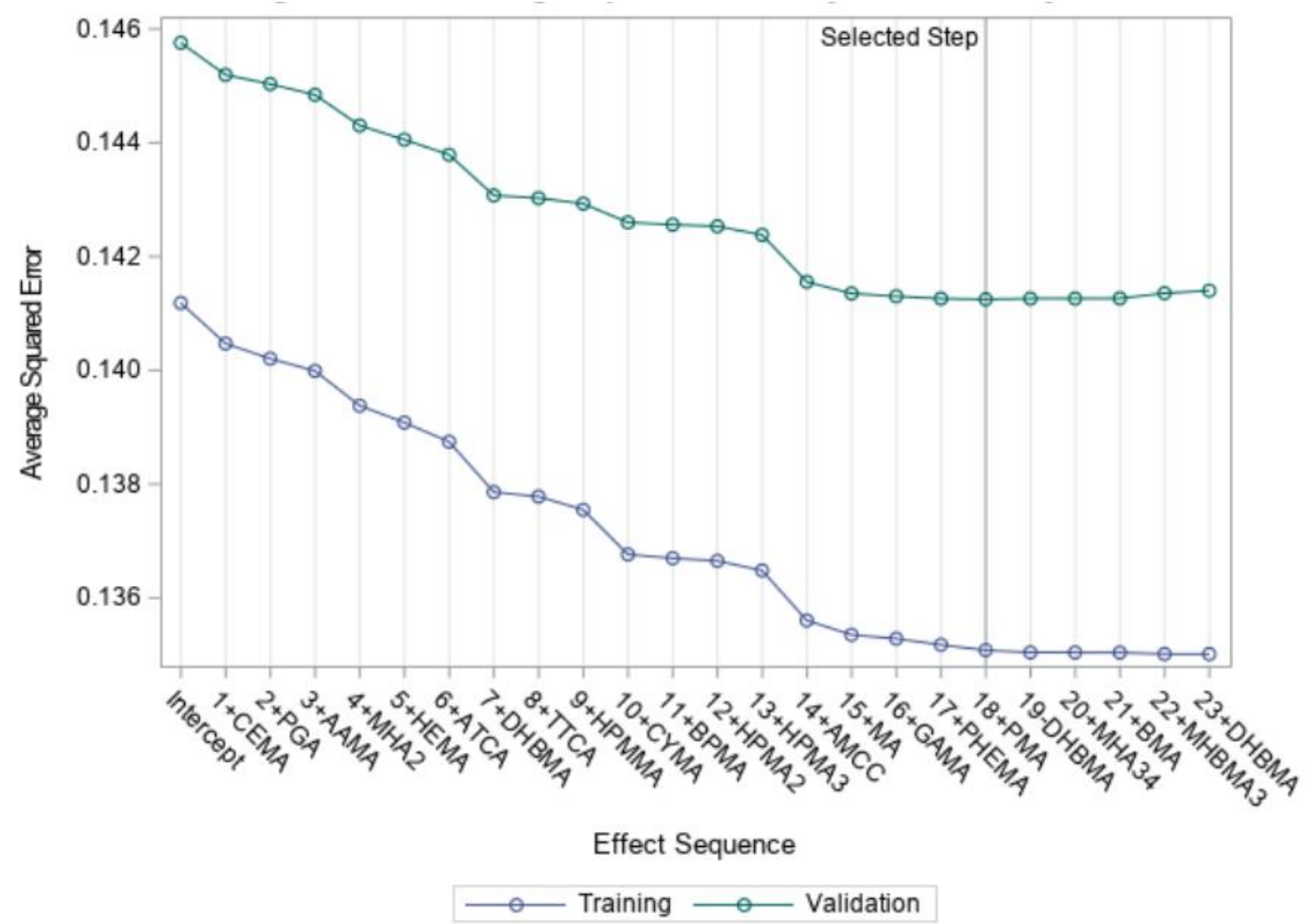

Figure 3.4.6. Training set and validation set errors plots for the generalized linear model employing the LASSO fit for $21 \mathrm{UM}$-VOCs in the full cohort.

Note: The vertical line labeled "selected step" is the model with the smallest $\mathrm{ASE}=$ best fit model. 


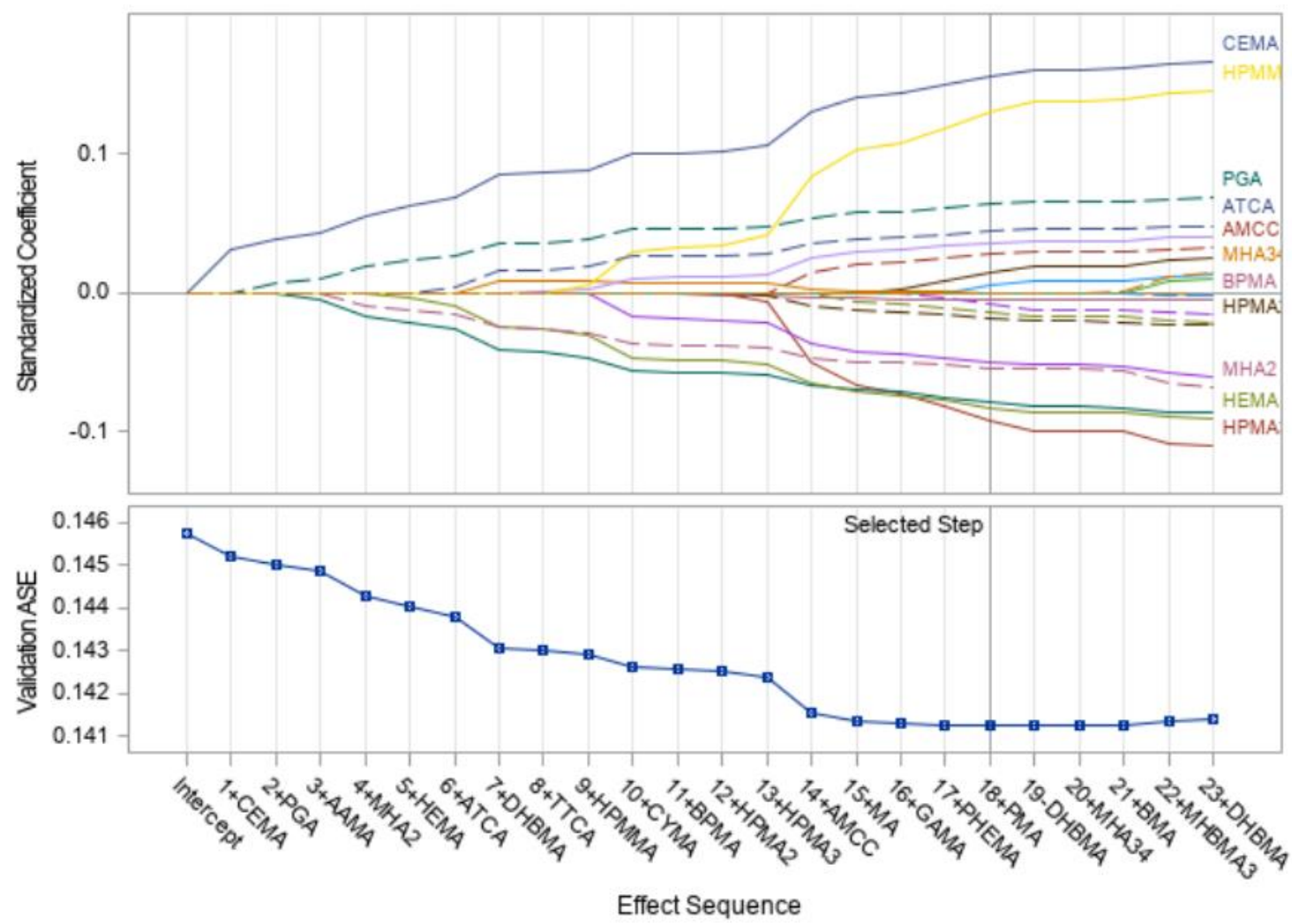

Figure 3.4.7 Coefficient Progression Plot- Standardized coefficients estimates as a function of the validations tuning parameter values for the generalized linear model employing the LASSO fit for $21 \mathrm{UM}-\mathrm{VOCs}$ in the full cohort.

Additionally, a linear model requesting the LASSO fit method for the full cohort was built using all 21 UM-VOCs and their two-way interactions. Again $30 \%$ of the data was reserved as validation data for tuning the model, which was used to estimate the prediction error for model selection; the remaining $70 \%$ of the data was used as the training data to fit the models. 232 variables were considered for selection which includes all two-way interactions.

Figure 3.4.8 shows the optimum model for predicting prevalent CMS using all 21 UM-VOCs and their two-way interactions (Model 30). The variables with explanatory value were chosen by the validation criteria in a generalized linear model employing the LASSO fit procedure for all $21 \mathrm{UM-VOCs}$ and all two-way 
interactions in the full study cohort can be observed in the coefficient progression plot (Figure 3.4.9). The UM-VOCs with most explanatory power in a linear model including all two-way interactions predicting prevalent CMS were 3HPMA and 2HPMA, and the two-way interactions retained in the model were: $3 \mathrm{HPMA}^{*} \mathrm{AAMA}$, AAMA ${ }^{*}$ CYMA, CEMA*DHBMA, AAMA*MHBMA3, BPMA*TTCA, CEMA*HPMMA, DHBMA*HPMMA, CEMA*ATCA, HEMA*ATCA, CEMA*AMCC, TTCA*AMCC, CEMA*PGA, HPMMA*PGA, PMA*PHEMA, CEMA*BMA, HEMA*BMA, AAMA*MHA2, PMA*2MHA.

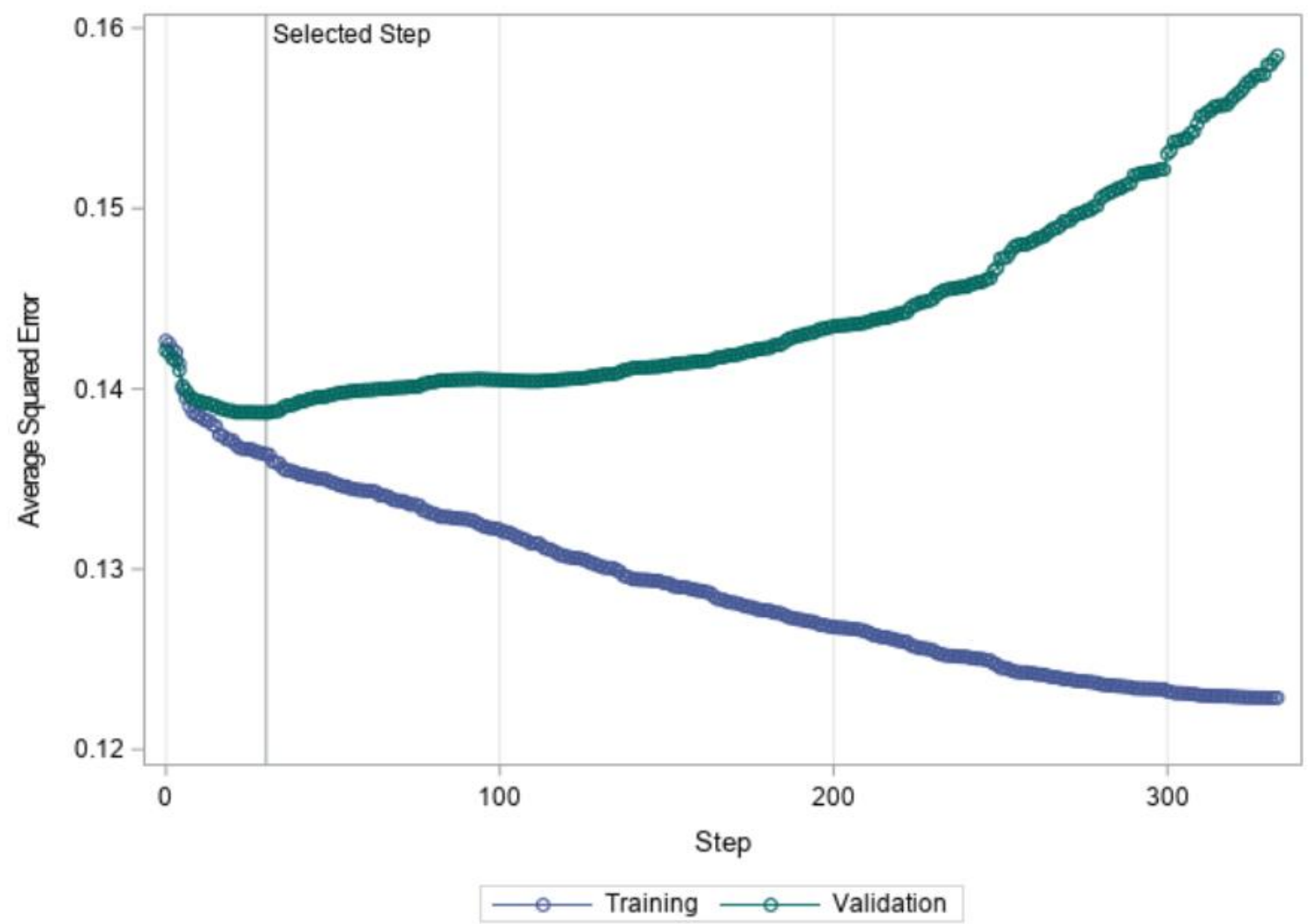

Figure 3.4.8. Training set and validation set errors for the generalized linear model employing the LASSO fit for $21 \mathrm{UM}$-VOCs and all two-way interactions in the full cohort.

Note: The vertical line labeled "selected step" is the model with the smallest ASE $=$ best fit model. 


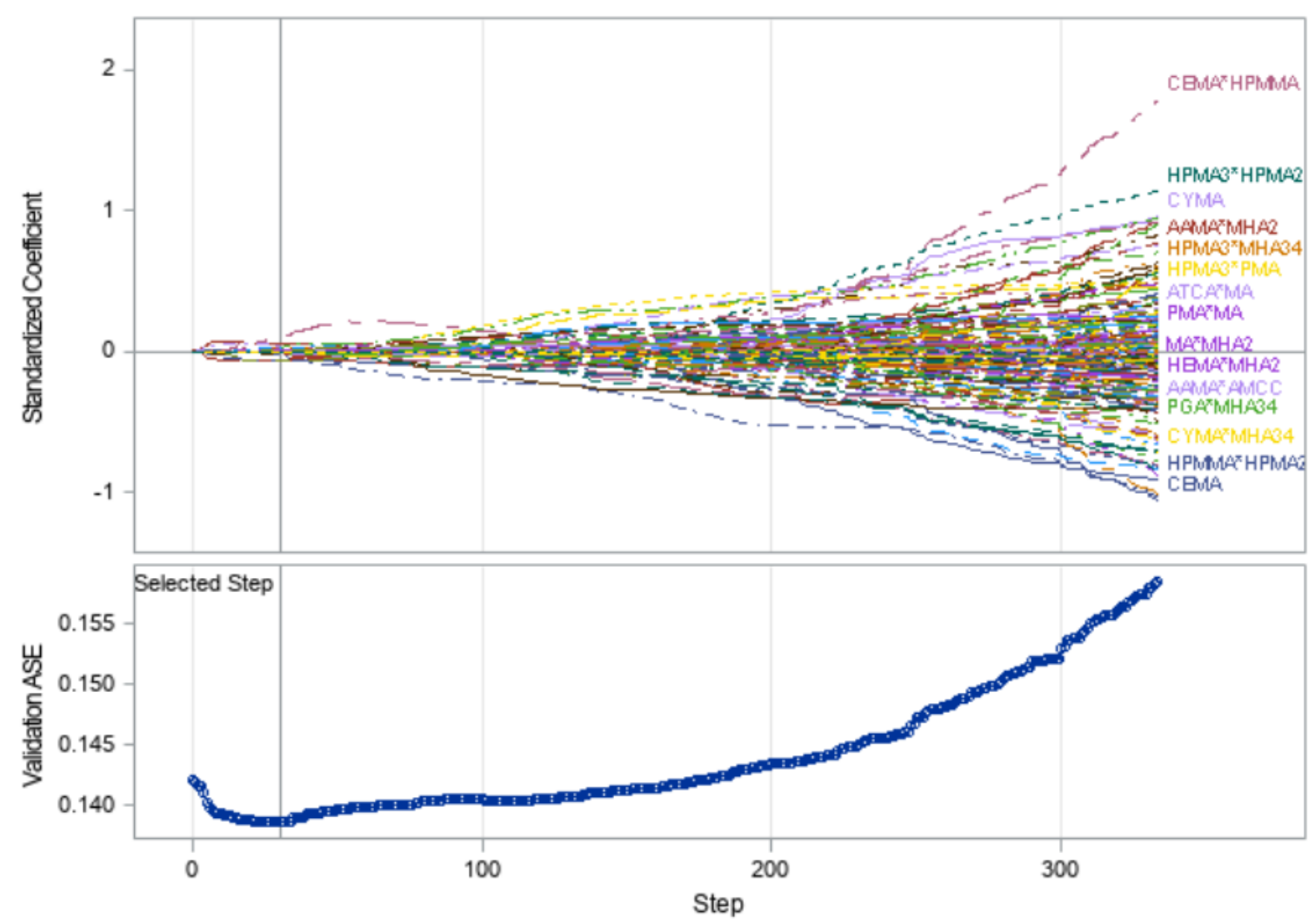

Figure 3.4.9 Coefficient Progression Plot- Standardized coefficients estimates as a function of the validations tuning parameter values for the generalized linear model employing the LASSO fit for $21 \mathrm{UM}$-VOCs and all two-way interactions in the full cohort.

\section{Cumulative VOC Exposure Score ( $\mathrm{UMM}-\mathrm{VOC})$}

A cumulative VOC exposure score was computed by adding quintile scores for thirteen representative UM-VOCs for each NHANES participant. The $\Sigma$ UMVOC exposure score was then used to identify if cumulative VOC exposures were associated with prevalent CMS and ordinal disease progression in non-tobacco smoke exposed participants. There were significant mean differences in the $\Sigma U M-$ VOC exposure score for all model adjustment variables (age, sex, race, and NHANES cohort year) (Table 3.4.3). 
Table 3.4.3 Mean $\Sigma U M-V O C$ exposure score for all adjustment variables in the $\Sigma \mathrm{UM}$-VOC exposure score logistic regression models for non-tobacco smoke exposed participants.

\begin{tabular}{llll}
\hline & \multicolumn{3}{c}{ Mean (SE) } \\
& \multicolumn{3}{c}{ EUM-VOC } \\
& $\mathrm{n}=2079$ Exposure & p-value \\
\hline Non-Smoke Exposed & & $38.12(0.50)$ & 0.017 \\
CMS & 388 & $39.93(0.99)$ & \\
No CMS & 1691 & $37.79(0.48)$ & \\
Age & 2079 & $38.12(0.59)$ & $<.0001$ \\
$\leq 51.0$ & 1076 & $36.10(0.57)$ & \\
$>51.0$ & 1103 & $40.63(0.63)$ & \\
Sex & 2079 & $38.12(0.50)$ & $<.0001$ \\
Male & 896 & $35.59(0.56)$ & \\
Female & 1183 & $40.04(0.59)$ & \\
Race or Ethnic Origin & 2079 & $38.12(0.50)$ & $<.0001$ \\
Mexican American & 364 & $38.62(0.74)$ & \\
Other Hispanic & 197 & $37.74(0.79)$ & \\
Non-Hispanic White & 921 & $38.52(0.62)$ & \\
Non-Hispanic Black & 333 & $34.31(0.65)$ & \\
Other/Multi Racial & 264 & $37.55(1.24)$ & \\
NHANES Cohort Year & 2079 & $38.12(0.50)$ & $<.0001$ \\
2005-2006 & 713 & $36.02(5.8)$ & \\
2011-2012 & 680 & $40.82(0.56)$ & \\
2013-2014 & 686 & $37.69(1.26)$ & \\
\hline
\end{tabular}

Note: 51.0 was the mean age of non-smokers

The mean $\Sigma \mathrm{UM}-\mathrm{VOC}$ exposure score for participants without CMS was $(37.79 \pm 0.48)$, while the mean $\Sigma U M-V O C$ exposure score for participants with CMS was (39.93 \pm 0.99$)$; representing a significant mean difference in cumulative VOC concentrations between individuals with and without CMS ( $p$-value $=0.0170$ ). For every one unit increase in the $\Sigma U M-V O C$ exposure score, the odds of having CMS increased by a factor of 1.020 (95\% Cl: 1.003, 1.037); however, after adjusting for age, sex, race, the interaction between sex and race, and NHANES cohort year, the association was no longer significant $(\mathrm{OR}=1.004 ; 95 \% \mathrm{Cl}: 0.988$, 
1.021). Additionally, the $\Sigma \cup M-V O C$ exposure score was significantly associated with the progression of disease towards clinically diagnosable CMS. There was a significant linear trend in the mean $\Sigma \mathrm{UM}-\mathrm{VOC}$ exposure score as the number of components of CMS increased ( $p$-value $=0.0018)($ Figure 3.4.10). An unadjusted ordinal logistic regression model also demonstrated a significant association between $\Sigma \mathrm{UM}-\mathrm{VOC}$ and CMS components treated ordinally $(\mathrm{OR}=1.015 ; 95 \% \mathrm{Cl}$ : $1.007,1.024)$. However, after adjusting for covariates, the association became attenuated (OR=0.996; 95\% Cl: 0.988, 1.004).

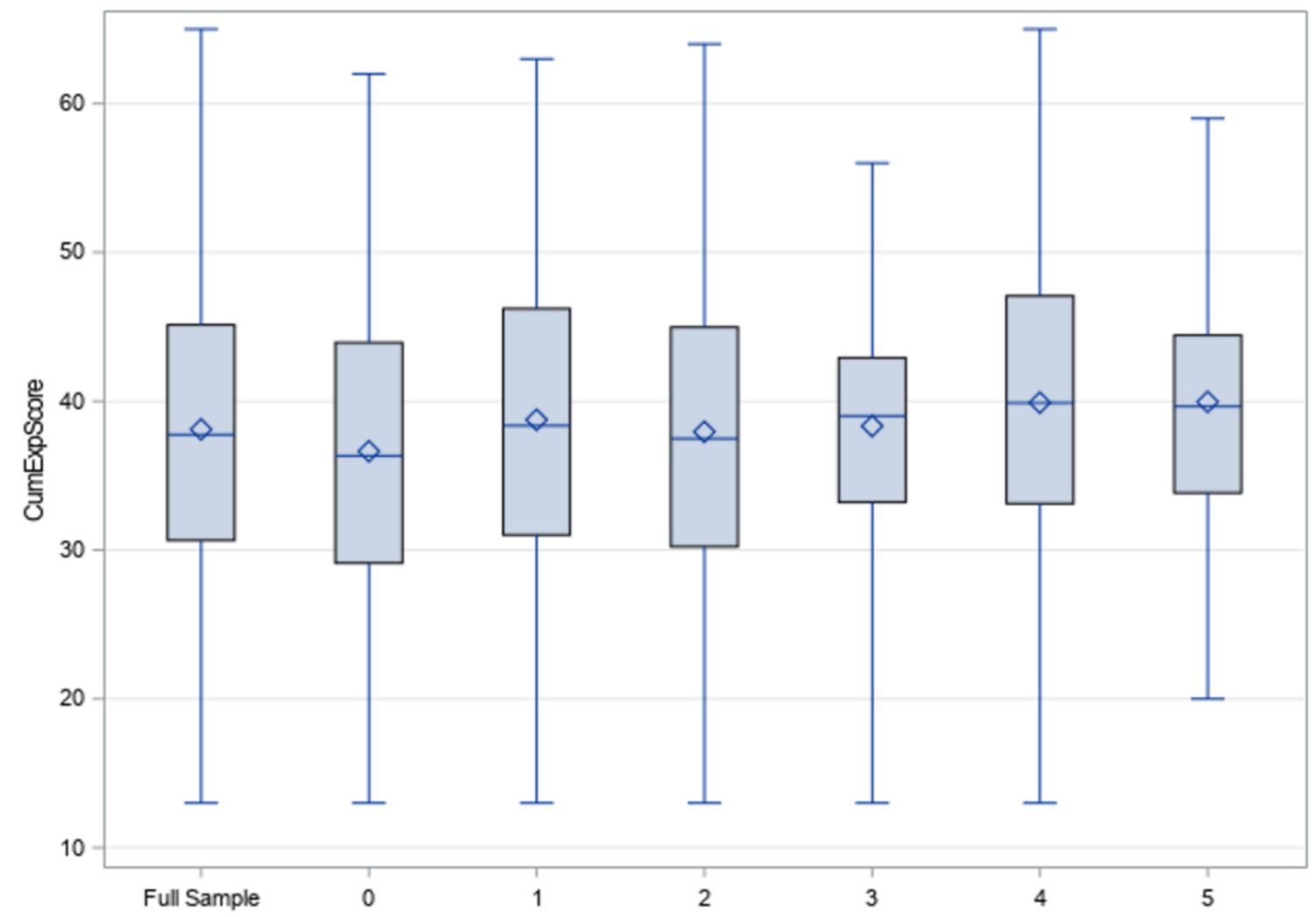

Figure 3.4.10. Box plot of $\Sigma U M-V O C$ exposure scores for the non-tobacco smoke exposed study cohort stratified by increasing number of components of CMS. 


\section{CHAPTER FOUR}

\section{SUMMARY AND DISCUSSION}

In this study nationally-representative associations of CMS and individuallevel concentrations of VOCs were examined, from both an individual VOC exposure perspective as well as a multipollutant exposure perspective. UM-VOC concentrations were significantly different between individuals with CMS compared with individuals without CMS and both prevalence and disease progression towards clinically diagnosable CMS was associated with UM-VOC concentrations. Furthermore, dose-response type relationships were observed with metabolites, supporting the hypothesis that CMS is positively associated with increasing UM-VOC concentrations. Taken together, these findings suggest that current general-population VOC exposures are of public health significance and may be playing a meaningful role in the increasing global CMS rates.

VOCs have been most widely studied with respect to their acute health effects or with respect to their carcinogenicity; however, exposure to VOCs is ubiquitous and the VOCs of interest in this study have previously been associated with numerous other adverse health effects. Additionally, in vivo investigations have established probable mechanistic links $(136,157,159,160)$; occupational 
studies have established significant associations with acute and chronic relativelyhigh-dose VOC exposures (141-143, 157, 158); and environmental and epidemiologic studies have provided significant evidence of associations between lower-dose chronic, and air pollution-related VOC exposures and CMS or components of CMS $(126,133)$. However, few studies have produced national estimates of personal-level VOC exposures. This research expands considerably on previous epidemiologic findings and provides support that relatively-low concentration, general-population VOC exposure levels are associated with CMS.

National-level distributions of personal-level measures of exposure to VOCs are lacking. This analysis is the first quantitative and representative investigation to use urinary metabolites of VOCs to assess associations with CMS. The majority of previous environmental and epidemiologic research demonstrating adverse health effects with VOCs have been conducted using exposure estimates generated with modeling techniques. The two most widely used modeling approaches are the bottom-up approach using emissions inventories and the topdown approach using ambient measures modeling (22, 245, 246). A limited number of studies have used other more individual-level exposure estimates such as personal air monitors and blood VOC measures $(225,230,247)$. Still fewer have used urinary metabolites as the measure of exposure(248-253).

Urinary metabolites of VOCs were used to estimate total VOC exposure in this analysis. These urinary metabolites of VOCs are measures of urinary mercapturic acids (MAs) and can be used as biomarkers of an individuals' internal toxicant dose (254). Excretion of MAs denotes electrophilic compound absorption 
or formation in the body from glutathione S-conjugates via the glutathione-pathway (235). Because glutathione is an essential endogenous compound used to protect against electrophiles and radicals, MAs reflect a toxicologically relevant absorbed dose (255). In comparison with blood VOC measures, the urinary metabolites of VOCs have fewer kinetic influences and have a relatively delayed clearance, resulting in measurements representative of a broader exposure window: minutes to hours for blood, versus hours to days for urine (256). Consequently, UM-VOCs better reflect an individual's day-to-day VOC exposure spectrum, and therefore UM-VOCs are able to capture indoor, personal-care, and volatile chemical products exposures, whose concentrations would quickly diminish in blood biomonitoring measurements.

Additionally, MAs have been used in environmental, biological, biological effects, and health surveillance monitoring for decades, and federal agencies have widely used MAs such as UM-VOCs as accurate, sensitive, and precise biomarkers of occupational and environmental exposures $(254,255,257,258)$. UM-VOCs have been used as biomarkers for tobacco-smoke exposure, and the detection and quantitation of numerous MAs has become an important tool for characterizing chemical exposures $(25,254,255)$. External measures of exposure to VOCs can present significant challenges. It has been shown that personal exposures are often greater than the sum of indoor and outdoor pollution levels, perhaps because fully accounting for the numerous routes and sources of exposure is extremely challenging (259). However, biomarker estimates better account for variations in toxicant absorption and metabolism which provide an 
individuals' internal toxicant dose (255).

Among the non-tobacco smoke exposed individuals investigated in this study, increased odds of CMS were found with metabolites of acrolein, 1,3butadiene, crotonaldehyde, cyanide, and ethylbenzene/styrene. Dose-response type relationships with metabolites of acrolein, 1,3-butadiene, and crotonaldehyde were observed. Acrolein and ethylbenzene/styrene metabolites were associated with disease progression towards clinically diagnosable CMS.

One of the major VOCs that was associated with CMS in this study was acrolein. Not only was acrolein associated with CMS dichotomously and ordinally in all sub-population analyses, but acrolein showed strong evidence of a doseresponse relationship with CMS. These patterns reflect the importance of acrolein exposures. The unwavering association between CEMA and CMS indicates the public health importance of even very low-dose exposures to acrolein. The relationship between CMS and acrolein at the population level suggest acrolein may be playing a role in the development or progression of CMS. Due to inadequate evidence, acrolein has not been classified as a carcinogen (41). However, acrolein is a known respiratory toxicant and has been liked to numerous adverse health effects including pulmonary edema, chronic obstructive pulmonary disease, and Alzheimer's $(41,260,261)$. More importantly for the support of this research, a limited number of studies have found acrolein is associated with increased risk of cardiovascular disease, atherosclerosis, diabetes, diabetic complications, and insulin resistance $(249,250,262-264)$. Furthermore, recent in vitro human endothelial cell work has shown acrolein exposure is associated with 
dysregulation of glucose transport and in vivo mice studies have begun to elucidate a potential mechanism $(265,266)$.

The mechanistic hypothesis for health effects associated with acrolein exposure involves the transient receptor potential ankyrin 1 (TRPA1) channel. TRPA1 is a non-selective cation (calcium) channel that is an uninhibited sensory receptor which mediates pain reception/transmission and irritant-related reflexes and is known to play a primary role in the inflammatory pain response (267). TRPA1 receptors are concentrated in sensory fibers but have also been identified in non-neuronal location, including the endothelium, urothelium, vasculature, and heart (268-277). The health effects hypothesis for acrolein exposure begins with acrolein activating the TRPA1 channel through aldehyde conjugation with free cysteines in the TRPA1 channel. The activation of the TRPA1 receptor incites an inflammatory/pain response known as 'neurogenic inflammation'. The neurogenic inflammation response induces the release of vasoactive peptides, substance P (a neuropeptide which acts as a neurotransmitter and as a neuromodulator), and calcitonin gene-related peptide, which increase blood flow, vascular permeability, and leukocyte binding $(269,278-285)$. Chronic systemic activation of the neurogenic inflammatory response would lead to endothelial dysfunction, proatherosclerotic changes, hypertension, and cardiometabolic dysfunction.

Crotonaldehyde and 1,3-butadiene were significantly associated with CMS in this study. Because crotonaldehyde is a major metabolite of 1,3-butadiene, it can be assumed that crotonaldehyde and 1,3-butadiene associations would likely be parallel and/or correlated. Like acrolein, crotonaldehyde is also an unsaturated 
aldehyde and preliminary unpublished work has shown crotonaldehyde produces similar TRPA1 neurogenic inflammatory effects as acrolein exposures (286). Furthermore, this study identified that acrolein had significant interactions with both 1,3-butadiene and crotonaldehyde in multipollutant model building analyses and all 3 VOCs were shared between factor 1 and 2 in the factor analysis, suggesting they explain a sizable amount of variance in both factors. Despite data showing the negative cardiovascular effects of unsaturated aldehydes are TRPA1dependent, the role of TRPA1 in the potential cardiometabolic toxicity of other aldehydes and mixtures is not well known (267, 287-289). The findings from this research support the hypothesis that the activation of the TRPA1 channel by unsaturated aldehydes, which is proposed to occur through aldehyde conjugation with the TRPA1 $\mathrm{N}$-terminal free cysteines, may induce cardiometabolic toxicity (290).

The ethylbenzene/styrene metabolite PGA was significantly associated with CMS in fully adjusted models as well as in tobacco smoke and non-tobacco smoke exposed subpopulation analyses. PGA was the only metabolite that was consistently associated with CMS in the tobacco smoke exposed subpopulation analyses. Ethylbenzene and styrene generate reactive oxygen species in the body. Styrene exposure has even been shown to increase lipid peroxidation and reactive oxygen species (ROS) formation in a dose-dependent manner $(291,292)$. ROS formation has also been shown to activate the TRPA1 channel $(271,293-295)$. While, the effect of styrene exposure on the general population at current levels of 
exposure is not known, findings in this study indicate an important link between ethylbenzene/styrene metabolites and CMS.

Acrolein, crotonaldehyde, and 1,3-butadiene were the three most associated VOCs with CMS. Evidence that all three activate neurogenic inflammation via the TRPA1 receptor has been reported. While, ethylbenzene/styrene metabolite activation of the TRPA1 receptor via ROS formation has not been explicitly studied ethylbenzene and styrene are known to generate high levels of oxidative stress and ROS has additionally been identified as an activator of the neurogenic inflammatory response via the TRPA1 receptor. Due to the cross-sectional nature of this study, evidence for causative links cannot be provided; however, the findings support speculation that chronic unsaturated aldehyde activation of the TRPA1 receptor is likely to be an important major contributor of the observed associations between VOCs and CMS.

In this study, differences between individuals exposed to tobacco smoke and those not exposed to tobacco smoke were explored. It has been well established that smokers and tobacco-smoke exposed individuals have significantly elevated levels of a number of the VOCs investigated in this study (25, 32, 33, 224, 296). Therefore, it was hypothesized that including individuals exposed to tobacco smoke would represent the high end of VOC exposure and that associations with VOCs and CMS would not only be maintained in the tobacco-smoke exposed individuals but would show greater associations. However, it was unexpected that individuals exposed to tobacco-smoke had few significant UM-VOCs associated with CMS. This lack of a relationship between 
VOC concentrations and CMS in tobacco smoke-exposed individuals has not been previously shown. While it was found that significant differences in the concentrations of VOCs existed between tobacco-smoke exposed and nontobacco smoke exposed individuals, there was not consistent evidence of a compelling relationship between VOC exposure and CMS in tobacco smoke exposed individuals. The inability to establish a relationship between VOCs and CMS in the tobacco smoke exposed subpopulation may be partially explained by the significantly higher VOC concentrations in the tobacco smoke-exposed individuals. The very high levels of VOCs in all individuals in this subpopulation make uncovering any association between UM-VOCs and CMS difficult. Factors, other than VOC exposure, are likely playing a larger role in determining the development of CMS among those exposed to tobacco smoke.

There are many strengths of this study. The study includes participants from three different NHANES cohorts and covers a relatively large span of time. The inclusion of multiple cohort years is noteworthy as it ensures that associations are steadfast and not identified by chance or mistake in one study population. The data analyzed come from a well-powered cohort representative of the entire United States. Few studies have assessed health effects of VOCs in a nationally representative manner. Still fewer have assessed these health effects from a multipollutant perspective. Several meaningful multipollutant groupings and interactions were found among UM-VOCs, and their associations with CMS. The logistic regression backward-selection method identified six significant two-way interactions between UM-VOC and CMS; whereas the LASSO penalized 
regression method, which is more equipped to handle the correlated exposures, identified eighteen significant two-way interactions. These two model-building techniques identifying such large numbers of two-way interactions emphasizes the importance of multipollutant approaches that consider the joint effects of multiple constituents. Furthermore, the cumulative VOC exposure score provided significant linear and unadjusted ordinal trends towards clinically diagnosable CMS, further illustrating the importance of the multipollutant mixtures perspective. Hence, the present study is the first to assess multipollutant health effects of VOCs using urinary metabolites as biomarkers of individual-level exposure to VOCs. The NHANES data are unique and valuable in providing a quantitative multi-year history of population exposures to VOCs in the U.S. Finally, the novel generation of a cumulative VOC exposure score provides unique insight into the nature and the associations of CMS and VOC exposures on a national level.

Nonetheless, the study has some limitations. First, all analytical methods of UM-VOC analysis in each NHANES waves were unchanged. However, upon close review of NHANES documentation, it was noted the 2005-2006 UM-VOC analysis was conducted on residual urine samples (NCHS, 2018). The 2005-2006 surplus urine samples were processed and then stored at less than $-20^{\circ} \mathrm{C}$ until samples were pulled for VOC analysis. Urinary metabolites are generally stable, and internal stability studies conducted by NHANES showed no evidence for metabolite degradation. The proper handling of samples, stability of the urinary VOC metabolites, consistency of analytical protocol, and internal validation studies provide a sufficient indication of the validity of the observed concentrations. 
A second limitation is the fact that inverse associations suggesting that increases in some UM-VOC concentrations are protective of CMS were observed in all subgroup analyses, in both adjusted and unadjusted models. Inverse associations between VOC exposures and health effects are not particularly plausible. VOCs are not likely to be protective of CMS status. It is possible that the metabolites associated with decreased odds of CMS are indicative of demographic subgroups with less CMS prevalence. For example, walking/cycling and home renovations have been identified as determinates of toluene exposure in a number of previous studies (247). Lifestyle factors such as physical activity and home renovations or new construction could be indicative of specific subpopulations with generally healthier lifestyles or overall better health for any number of reasons.

It has been well established that benzene is highly correlated with exposure to tobacco smoke and is a known carcinogen, endocrine disruptor and has been previously linked to cardiometabolic disease $(50,63,247,297)$. Findings that benzene is inversely associated with CMS in the tobacco smoke exposed subpopulation is suspect. It is possible that confounding may have played a role in the inverse associations identified with VOCs and CMS. Some evidence of confounding by age was present in this analysis. The inverse associations between UM-VOCs and CMS identified among the tobacco smoke exposed populations of this study warrant further investigation.

Finally, uncertainties arise from the lack of information on actual exposures and individual variation including genetic and physiological differences that likely lead to variation in rates of VOC metabolism. The analysis showed age, sex, and 
race were important variables to adjust for in models. Age, sex, and race each influence metabolism but individual variation or subgroup variation characterizations have not been well studied or defined. As the field of metabolomics continues to evolve, attention to the subgroup variations and the ability to handle these discrepancies will undoubtedly need special consideration. There is sufficient warrant to conduct more detailed exposure assessments and metabolomic analyses to identify new potential factors responsible for variations within and across populations. Once identified, these data could be added to future studies to aid in the identification of the major contributors of subpopulation differences in VOCs to help improve future analyses.

Additional work is also needed to assess VOC exposures geographically and by urban/rural subgroups. It is currently unknown if or to what extent spatial variability exists in personal exposure to VOCs; however, the observed associations between VOCs and CMS in the United States, identified in this investigation, are not likely to be solely attributed to this variation. Nevertheless, the increasing proportion of people living in urban environments may be an important contributing factor. Additional analyses of other cardiometabolic health related effects and updated economic implications of national level health effects associated with VOC exposures is needed. It is probable that the direct and indirect economic losses accrued over several generations could outweigh the capital investments needed for the implementation of volatile organic compoundreduction initiatives. These analyses would strengthen the rationale for the 
importance of national VOC regulations particularly for environmental VOC regulations. 


\section{CONCLUSION}

Recent work suggests that exposure to environmental air pollution is an important contributor to cardiovascular and metabolic dysfunction. Exceedingly little is known about the extent to which environmental air pollution exposures contribute to cardiometabolic syndrome or if VOCs are an important contributor to insulin resistance and cardiometabolic syndrome. Exposure and disease rate associations are important to study as their implications are significant for public health, clinical and mechanistic advances.

The importance of mixtures investigations in exposure science has recently been underscored by academic researchers and policy makers. Numerous singleexposure-type investigations of the health effects of VOCs have been conducted but to date only a handful of investigations have assessed multipollutant VOC exposures and their associations with health effects; this is the first investigation to study the combinatory effects of these specific VOCs using urinary metabolites, or to investigate mixtures of these UM-VOCs and their associations with CMS.

The findings from this research are an important step in elucidating the importance of VOCs' contributions to chronic disease processes, specifically CMS. Information regarding the links between VOC and CMS would strongly reinforce priorities in public health policy and planning and incentives for pursuing specific strategies to change unhealthy environmental contributions and ultimately reduce cardiometabolic health-related burdens. This investigation of the associations 
between VOCs with CMS have provided fundamental insight into the contribution of a major modifiable environmental pollutant and its role in CMS.

These novel nationally-representative estimates of personal-level exposure to VOCs derived from individual participants' urinary metabolites provide unique insight into associations and patterns of personal exposure to VOCs and their associations with CMS in the United States. To date, quantitative and nationally representative associations of individual-level exposure to VOCs using urinary biomarkers of exposure and their associations with CMS prevalence have not been reported.

In conclusion, it was hypothesized that increasing levels of UM-VOCs would be positively associated with an increase in the prevalence of CMS. Significant associations were found with a number of VOCs, namely acrolein, 1,3-butadiene, crotanaldehyde, ethylbenzene and styrene, and CMS. The heart and vascular system are highly vulnerable structures. There are a multitude of vehicles for injury to these systems as well as a multitude of outcomes associated with these injuries. The specific processes of these injuries are important to define. Cardiometabolic syndrome is an amalgamation of these processes of injuries and the resulting conditions. These findings significantly contribute to the growing body of evidence demonstrating the importance of chronic neurogenic inflammation via the activations of the TRPA1 receptor, one likely mechanism of VOC-induced health effects. 


\section{REFERENCES}

1. Castro JP, El-Atat FA, McFarlane SI, et al. Cardiometabolic syndrome: pathophysiology and treatment. Curr Hypertens Rep 2003;5(5):393-401.

2. Statistics CfDCaPNCfH. CDC WONDER: multiple cause of death 19992017. December 2018.; 2018. (http://wonder.cdc.gov/mcd-icd10.html). (Accessed January 12020 ).

3. Organization WH. Noncommunicable diseases country profiles 2018. Geneva, 2018.

4. Murray CJ, Atkinson C, Bhalla K, et al. The state of US health, 1990-2010: burden of diseases, injuries, and risk factors. Jama 2013;310(6):591-608.

5. Govindarajan G, Whaley-Connell A, Mugo M, et al. The cardiometabolic syndrome as a cardiovascular risk factor. The American journal of the medical sciences 2005;330(6):311-8.

6. Jardim TV, Mozaffarian D, Abrahams-Gessel S, et al. Cardiometabolic disease costs associated with suboptimal diet in the United States: A cost analysis based on a microsimulation model. PLOS Med 2019;16(12):e1002981.

7. Vincent GE, Jay SM, Sargent C, et al. Improving Cardiometabolic Health with Diet, Physical Activity, and Breaking Up Sitting: What about Sleep? Front Physiol 2017;8:865.

8. Landrigan PJ, Fuller R, Acosta NJR, et al. The Lancet Commission on pollution and health. Lancet 2018;391(10119):462-512.

9. Organization) WWH. Burden of disease from the joint effects of Household and Ambient Air Pollution for 2012- Description of Method. Geneva, Switzerland: World Health Organization; 2016.

(www.who.int/phe/health_topics/outdoorair/databases/AP_jointeffect_BoD _results_Nov2016.pdf). (Accessed 14 February 2019).

10. Konkle SL, Zierold KM, Taylor KC, et al. National secular trends in ambient air volatile organic compound levels and biomarkers of exposure in the United States. Environmental research 2019;182:108991. 
11. Dockery DW, Pope CA, 3rd, Xu X, et al. An association between air pollution and mortality in six U.S. cities. The New England journal of medicine 1993;329(24):1753-9.

12. Jerrett M, Burnett RT, Pope CA, 3rd, et al. Long-term ozone exposure and mortality. The New England journal of medicine 2009;360(11):1085-95.

13. Jimenez JL, Canagaratna MR, Donahue NM, et al. Evolution of organic aerosols in the atmosphere. Science 2009;326(5959):1525-9.

14. Brook RD, Rajagopalan S, Pope CA, 3rd, et al. Particulate matter air pollution and cardiovascular disease: An update to the scientific statement from the American Heart Association. Circulation 2010;121(21):2331-78.

15. Pope CA, 3rd, Burnett RT, Thurston GD, et al. Cardiovascular mortality and long-term exposure to particulate air pollution: epidemiological evidence of general pathophysiological pathways of disease. Circulation 2004;109(1):71-7.

16. Di Q, Wang Y, Zanobetti A, et al. Air Pollution and Mortality in the Medicare Population. The New England journal of medicine 2017;376(26):2513-22.

17. Bell ML, Ebisu K, Peng RD, et al. Hospital admissions and chemical composition of fine particle air pollution. American journal of respiratory and critical care medicine 2009;179(12):1115-20.

18. Franklin $\mathrm{M}$, Koutrakis $\mathrm{P}$, Schwartz $\mathrm{P}$. The role of particle composition on the association between PM2.5 and mortality. Epidemiology 2008;19(5):680-9.

19. Laden F, Neas LM, Dockery DW, et al. Association of fine particulate matter from different sources with daily mortality in six U.S. cities. Environmental health perspectives 2000;108(10):941-7.

20. Ostro B, Feng WY, Broadwin R, et al. The effects of components of fine particulate air pollution on mortality in california: results from CALFINE. Environmental health perspectives 2007;115(1):13-9.

21. Zanobetti $\mathrm{A}$, Franklin $\mathrm{M}$, Koutrakis $\mathrm{P}$, et al. Fine particulate air pollution and its components in association with cause-specific emergency admissions. Environmental health : a global access science source 2009;8:58.

22. Agency) USEUSEP. 2014 National Emissions Inventory Report. 2016. (https://www.epa.gov/air-emissions-inventories/2014-national-emissionsinventory-nei-data). (Accessed 12 February 2019). 
23. Registry) AAfTSaD. ATSDR's Substance Priority List. www.atsdr.cdc.gov: Agency for Toxic Substances and Disease Registry; 2017. (https://www.atsdr.cdc.gov/spl/\#2017spl). (Accessed 3 April 2019).

24. Kirk EP, Klein S. Pathogenesis and pathophysiology of the cardiometabolic syndrome. J Clin Hypertens (Greenwich) 2009;11(12):761-5.

25. Alwis KU, Blount BC, Britt AS, et al. Simultaneous analysis of 28 urinary VOC metabolites using ultra high performance liquid chromatography coupled with electrospray ionization tandem mass spectrometry (UPLCESI/MSMS). Anal Chim Acta 2012;750:152-60.

26. Technical Overview of Volatile Organic Compounds. EPA.gov: EPA; 2017. (https://www.epa.gov/indoor-air-quality-iaq/technical-overview-volatileorganic-compounds). (Accessed April 182017 2017).

27. Publications ADMaAWBS. IUPAC. Compendium of Chemical Terminology, (the "Gold Book"). Oxford 1997.

28. Rubin, J. I., A. J. Kean, R. A. Harley, D. B. Millet, and A. H. Goldstein (2006), Temperature dependence of volatile organic compound evaporative emissions from motor vehicles, J. Geophys. Res., 111, D03305, doi:10.1029/2005JD006458.

29. Sillman, S., and P. J. Samson (1995), Impact of temperature on oxidant photochemistry in urban, polluted rural and remote environments, J. Geophys. Res., 100(D6), 11,497-11,508.

30. Sexton K, Adgate JL, Church TR, et al. Children's exposure to volatile organic compounds as determined by longitudinal measurements in blood. Environmental health perspectives 2005;113(3):342-9.

31. Handy R, D. Smith, N. Castillo, C. Sparacino, AND K. Thomas. Total Exposure Assessment Methodology (TEAM) Study: Standard Operating Procedures Employed In Support Of An Exposure Assessment Study. Washington, D.C., EPA/600/6-87/002D, (Agency USEP

32. Jain RB. Distributions of selected urinary metabolites of volatile organic compounds by age, gender, race/ethnicity, and smoking status in a representative sample of U.S. adults. Environ Toxicol Pharmacol 2015;40(2):471-9.

33. Chambers DM, Ocariz JM, McGuirk MF, et al. Impact of cigarette smoking on volatile organic compound (VOC) blood levels in the U.S. population: NHANES 2003-2004. Environment international 2011;37(8):1321-8. 
34. Volatile Organic Compounds Emissions. EPA.gov: EPA; 2014. (https://cfpub.epa.gov/roe/indicator.cfm?i=23). (Accessed June 19 2017).

35. Guenther A, Hewitt, C. N., Erickson, D., Fall, R., Geron, C., Graedel, T., Harley, P., Klinger, L., Lerdau, M., McKay, W. A., Pierce, T., Scholes, B., Steinbrecher, R., Tallamraju, R., Tay- lor, J., and Zimmerman, P. A global model of natural volatile organic compound emissions. Geophys Res 1995.

36. Pekka Rantala LJr, Risto Taipale, Terhi K. Laurila, Johanna Patokoski, Maija K. Kajos, Mona Kurppa, Sami Haapanala, Erkki Siivola, Tuukka Petäjä, Taina M. Ruuskanen, and Janne Rinne. Anthropogenic and biogenic influence on VOC fluxes at an urban background site in Helsinki, Finland. Atmos Chem Phys 2016.

37. CIESIN (CENTER FOR INTERNATIONAL EARTH SCIENCE INFORMATION NETWORK), 2004, Global Rural-Urban Mapping Project (GRUMP), Alpha Version: Urban Extents. Available online at: http://sedac.ciesin.columbia.edu/gpw (accessed 16 October 2017).

38. Canada Go. Toxic Substances List - Schedule 1. www.ec.gc.ca: Government of Canada, 2017, (Canada EaCC

39. Code of Federal Regualtions, 40: Chapter 1, Subchapter C, Part 51, Subpart f, 51100 .

40. Registry) AAfTSaD. Agency for Toxic Substances and Disease Registry. atsdr.cdc.gov: U.S. Department of Health \& Human Services; 2019. (https://www.atsdr.cdc.gov). (Accessed 12 May 2019).

41. Registry AfTSaD. Toxicological Profile for Acrolein. atsdr.cdc.gov: CDC; 2007. (https://www.atsdr.cdc.gov/toxprofiles/tp.asp?id=557\&tid=102). (Accessed 2017).

42. Information. NCfB. PubChem Compound Database; Acrolein, CID 7847. nih.gov; 2004. (https://pubchem.ncbi.nlm.nih.gov/compound/7847\#section=Top). (Accessed May 2017).

43. Information NCfB. PubChem Compound Database; Acrylamide CID 6579. nih.gov; 2004. (https://pubchem.ncbi.nlm.nih.gov/compound/6579). (Accessed 2017).

44. Registry AfTSaD. Toxicological Profile for Acrylamide. atsdr.cdc.gov: CDC; 2012.

(https://www.atsdr.cdc.gov/toxprofiles/tp.asp?id=1112\&tid=236). (Accessed 2017). 
45. Administration OSaH. Permissible Exposure Limits. osha.gov United States Department of Labor, (Labor USDo

46. Ford ES, Li C, Zhao G. Prevalence and correlates of metabolic syndrome based on a harmonious definition among adults in the US. Journal of diabetes 2010;2(3):180-93.

47. Information NCfB. PubChem Compound Database; Acrylonitrile, CID 7855. nih.gov; 2014.

(https://pubchem.ncbi.nlm.nih.gov/compound/7855\#section=Top). (Accessed June 2017).

48. Registry AfTSaD. Toxicological Profile for Acrylonitrile. atsdr.cdc.gov: CDC; 1990. (https://www.atsdr.cdc.gov/toxprofiles/tp.asp?id=447\&tid=78). (Accessed June 2017).

49. USEPA Office of Pesticide Programs HED, Science Information Management Branch: . Chemicals Evaluated for Carcinogenic Potential by the Office of Pesticide Programs. 2006. (http://www.fluoridealert.org/wpcontent/pesticides/pesticides.cancer.potential.2006.pdf). (Accessed June 2017).

50. Cancer IAfRo. IARC Monograohs on the Evaluation of Carcinogenic Risks to Humans World Health Organization 2017.

(http://monographs.iarc.fr/ENG/Classification/index.php). (Accessed 2017).

51. Simonneau G, Robbins IM, Beghetti M, et al. Updated clinical classification of pulmonary hypertension. J Am Coll Cardiol 2009;54(1 Suppl):S43-54.

52. Registry AfTSaD. Toxicological Profile for Vinyl Chloride. atsdr.cdc.gov: CDC; 2004.

(https://www.atsdr.cdc.gov/toxprofiles/TP.asp?id=282\&tid=51). (Accessed June 2017).

53. Information NCfB. PubChem Compound Database; Vinyl chloride, CDI 6338. nih.gov; 2004.

(https://pubchem.ncbi.nlm.nih.gov/compound/6338\#section=Top). (Accessed June 2017).

54. Lender D, Sysko SK. The metabolic syndrome and cardiometabolic risk: scope of the problem and current standard of care. Pharmacotherapy 2006;26(5 Pt 2):3S-12S.

55. Information NCfB. PubChem Compound Database; Oxirane, CID 6354. nih.gov; 2004. 
(https://pubchem.ncbi.nlm.nih.gov/compound/6354\#section=Top). (Accessed June 2017).

56. Registry AfTSaD. Toxicological Profile for Ethylene Oxide atsdr.cdc.gov: CDC; 1990. (https://www.atsdr.cdc.gov/PHS/PHS.asp?id=732\&tid=133). (Accessed June 2017).

57. National Toxicology Program DoHaHS. Ethylene Oxide. nih.gov. (https://ntp.niehs.nih.gov/ntp/roc/content/profiles/ethyleneoxide.pdf). (Accessed June 2017).

58. Manrique C, Lastra G, Whaley-Connell A, et al. Hypertension and the cardiometabolic syndrome. J Clin Hypertens (Greenwich) 2005;7(8):471-6.

59. Kim S, Thiessen PA, Bolton EE, et al. PubChem Substance and Compound databases. Nucleic acids research 2016;44(D1):D1202-13.

60. Wilbur S, Wohlers D, Paikoff S, et al. ATSDR evaluation of potential for human exposure to benzene. Toxicology and industrial health 2008;24(56):399-442.

61. National emission standards for hazardous air pollutants for ethylene oxide commercial sterilization and fumigation operations--EPA. Interim final rule. Fed Regist 1998;63(233):66990-4.

62. Wallace L. Environmental exposure to benzene: an update. Environmental health perspectives 1996;104 Suppl 6:1129-36.

63. CDC. Glossary of Volatile Organic Compounds. CDC.gov: Centers for Disease Control and Prevention (https://www.cdc.gov/nceh/clusters/Fallon/Glossary-VOC.pdf). (Accessed).

64. Wilbur S, Wohlers D, Paikoff S, et al. ATSDR evaluation of health effects of benzene and relevance to public health. Toxicology and industrial health 2008;24(5-6):263-398.

65. (IRIS) IRIS. Chemical Assessment Summary- Benzene; CASRN 71-43-2. U.S. Environmental Protection Agency.

66. Statistics NCfH. National Health and Nutrition Examination Survey (NHANES)- MEC Laboratory Procedures Manual 2013.

67. Information NCfB. PubChem Compound Database; 1-Bromopropane, CID 7840. nih.gov; 2005.

(https://pubchem.ncbi.nlm.nih.gov/compound/7840\#section=Top). (Accessed June 2017). 
68. Registry AfTSaD. Toxicological Profile for 1-Bromopropane. atsdr.cdc.gov: CDC; 2016.

(https://www.atsdr.cdc.gov/toxprofiles/tp.asp?id=1471\&tid=285). (Accessed June 2017).

69. Agency EC. Agreement Of The Member State Committee On The Identification Of 1-Bromopropane As A Substance Of Very High Concern. Articles 57 and 59 of Regulation (EC) 1907/2006, 2012.

70. Cutler JA, Sorlie PD, Wolz M, et al. Trends in hypertension prevalence, awareness, treatment, and control rates in United States adults between 1988-1994 and 1999-2004. Hypertension 2008;52(5):818-27.

71. Information NCfB. PubChem Compound Database; 13-Butadiene, CID 7845. nih.gov; 2005.

(https://pubchem.ncbi.nlm.nih.gov/compound/7845\#section=Top). (Accessed June 2017).

72. Registry AfTSaD. Toxicological Profile for 1,3-Butadiene. atsdr.cdc.gov: CDC; 2012. (https://www.atsdr.cdc.gov/PHS/PHS.asp?id=457\&tid=81). (Accessed June 2017).

73. Toxics EA. Health Assessment of 1,3-Butadiene. epa.gov; 2002. (https://cfpub.epa.gov/si/si_public_record_report.cfm?direntryid=54499). (Accessed June 2017).

74. Ford ES. Prevalence of the metabolic syndrome defined by the International Diabetes Federation among adults in the U.S. Diabetes care 2005;28(11):2745-9.

75. Information NCfB. PubChem Compound Database; Carbon Disulfide, CID 6348. nih.gov; 2004.

(https://pubchem.ncbi.nlm.nih.gov/compound/6348\#section=Top). (Accessed July 2017).

76. Registry AfTSaD. Toxicological Profile for Carbon Disulfide atsdr.cdc.gov: CDC; 1996. (https://www.atsdr.cdc.gov/toxprofiles/tp.asp?id=474\&tid=84). (Accessed July 2017).

77. Klaassen CD. Casarett and Doull's Toxicology. The Basic Science of Poisons. New York, NY: McGraw-Hill; 2001.

78. Gosselin RE, R.P. Smith, H.C. Hodge. Clinical Toxicology of Commercial Products. 5th ed.: Baltimore: Williams and Wilkins; 1984.

79. Eckel $\mathrm{RH}$, Kahn R, Robertson RM, et al. Preventing cardiovascular disease and diabetes A call to action from the American Diabetes 
Association and the American Heart Association. Circulation 2006;113(25):2943-6.

80. Toxicological Profile for Crotonaldehyde. atsdr.cdc.gov: CDC; 2002. (https://www.atsdr.cdc.gov/toxfaqs/tf.asp?id=948\&tid=197). (Accessed July 2017).

81. Information NCfB. PubChem Compound Database; 1-Bromopropane, CID 447466. nih.gov; 2005.

(https://pubchem.ncbi.nlm.nih.gov/compound/447466\#section=Top). (Accessed July 2017).

82. Chung FL, Tanaka T, Hecht SS. Induction of liver tumors in F344 rats by crotonaldehyde. Cancer research 1986;46(3):1285-9.

83. (IRIS) USEPAsIRIS. Summary on Crotonaldehyde; CASRN 123-73-9. 1991. (http://www.epa.gov/iris/ ). (Accessed July 2017).

84. BEls ACoGIHTa. Threshold Limit Values for Chemical Substances and Physical Agents and Biological Exposure Indices. Cincinnati, $\mathrm{OH}$, 2008:22.

85. Registry AfTSaD. Toxicological Profile for Cyanide. atsdr.cdc.gov: CDC; 2006. (https://www.atsdr.cdc.gov/toxfaqs/tf.asp?id=71\&tid=19). (Accessed July 2017).

86. Pritsos CA, Ma J. Biochemical assessment of cyanide-induced toxicity in migratory birds from gold mining hazardous waste ponds. Toxicology and industrial health 1997;13(2-3):203-9.

87. Ibebunjo C, Kamalu BP, Ihemelandu EC. Comparison of the effects of cassava (Manihot esculenta Crantz) organic cyanide and inorganic cyanide on muscle and bone development in a Nigerian breed of dog. The British journal of nutrition 1992;68(2):483-91.

88. Neves MF, Virdis A, Sanjuliani AF, et al. Hypertension and cardiometabolic risk factors. Int J Hypertens 2013;2013:634798.

89. Information NCfB. PubChem Compound Database; Oxirane, CID 6228. nih.gov; 2004.

(https://pubchem.ncbi.nlm.nih.gov/compound/6228\#section=Top). (Accessed July 2017).

90. National Research Council Committee on Acute Exposure Guideline L. Acute Exposure Guideline Levels for Selected Airborne Chemicals. Washington, DC: The National Academies Press, 2011. 
91. G. Long and M.E. Meek ML. Concise International Chemical Assessment; N, N-Dimethylformamide Geneva: World Health Organization, 2001.

92. Network TD. N,N-DIMETHYLFORMAMIDE. nih.gov NIH; 2014. (https://toxnet.nlm.nih.gov/cgibin/sis/search/a?dbs+hsdb:@term+@DOCNO+78). (Accessed July 2017).

93. Information NCfB. PubChem Compound Database; Ethylbenzene, CID 7500. nih.gov: NIH; 2004.

(https://pubchem.ncbi.nlm.nih.gov/compound/7500\#section=Top). (Accessed July 2017).

94. Registry AfTSaD. Toxicological Profile for Ethylbenzene. atsdr.cdc.gov: CDC; 2016. (https://www.atsdr.cdc.gov/PHS/PHS.asp?id=381\&tid=66). (Accessed July 2017).

95. Bingham EC, B.; Powell, C.H. Patty's Toxicology. New York, N.Y.: John Wiley \& Sons; 2001.

96. Burt VL, Whelton P, Roccella EJ, et al. Prevalence of hypertension in the US adult population. Results from the Third National Health and Nutrition Examination Survey, 1988-1991. Hypertension 1995;25(3):305-13.

97. Information NCfB. PubChem Compound Database; Stryene, CID 7501. nih.gov: NIH; 2004.

(https://pubchem.ncbi.nlm.nih.gov/compound/7501\#section=Top). (Accessed July 2017).

98. Registry AfTSaD. Toxicological Profile for Styrene. atsdr.cdc.gov: CDC; 2012. (https://www.atsdr.cdc.gov/PHS/PHS.asp?id=419\&tid=74). (Accessed July 2017).

99. Shen S, Zhang F, Gao L, et al. Detection of phenolic metabolites of styrene in mouse liver and lung microsomal incubations. Drug Metab Dispos 2010;38(11):1934-43.

100. Prieto-Castello MJ, Cardona A, Marhuenda D, et al. Use of the CYP2E1 genotype and phenotype for the biological monitoring of occupational exposure to styrene. Toxicology letters 2010;192(1):34-9.

101. Ford ES, Giles WH, Mokdad AH. Increasing prevalence of the metabolic syndrome among u.s. Adults. Diabetes care 2004;27(10):2444-9.

102. Agency USEP. Propylene oxide. epa.gov: EPA; 1992. (https://www3.epa.gov/ttn/atw/). (Accessed July 2017).

103. Agency USEP. Summary Review of the Health Effects Associated with Propylene Oxide. Environmental Criteria and Assessment Office, Office of 
Health and Environmental Assessment, Office of Research and Development, Research Triangle Park, NC., 1987.

104. Budavari. ES. The Merck Index An Encyclopedia of Chemicals, Drugs, and Biologicals. 11th ed. Rahway, NJ.: Merck and Co. Inc.; 1989.

105. Information NCfB. PubChem Compound Database; Tetrachloroethene, CID 31373. nih.gov: NIH; 2004. (https://pubchem.ncbi.nlm.nih.gov/compound/31373\#section=Top). (Accessed July 2017).

106. Agency USEP. Tetrachloroethylene (Perchloroethylene). epa.gov: EPA, 2000 ,

107. Registry AfTSaD. Toxicological Profile for Tetrachloroethylene atsdr.cdc.gov: CDC; 2015.

(https://www.atsdr.cdc.gov/PHS/PHS.asp?id=263\&tid=48). (Accessed July 2017).

108. Committee to Review EPA's Toxicological Assessment of Tetrachloroethylene BoESaT, Division on Earth and Life Studies. Review of the Environmental Protection Agency's Draft IRIS Assessment of Tetrachloroethylene. 500 Fifth Street, NW Washington, DC 20001, 2010:186.

109. Ford ES, Giles WH. A comparison of the prevalence of the metabolic syndrome using two proposed definitions. Diabetes care 2003;26(3):57581.

110. Information NCfB. PubChem Compound Database; CID, 1140 NIH.gov; 2004. (https://pubchem.ncbi.nlm.nih.gov/compound/1140). (Accessed May 2017).

111. Toxic Substances Portal. Centers for Disease Control and Prevention. 2011 ; (https://www.atsdr.cdc.gov/substances/toxsubstance. asp?toxid=29). (Accessed May 9, 2017).

112. Registry AfTSaD. Toxicological Profile for Toluene atsdr.cdc.gov: CDC; 2016. (https://www.atsdr.cdc.gov/toxprofiles/tp.asp?id=161\&tid=29). (Accessed 2017).

113. Troiano RP, Flegal KM. Overweight children and adolescents: description, epidemiology, and demographics. Pediatrics 1998;101(3 Pt 2):497-504.

114. Information NCfB. PubChem Compound Database; Trichloroethene, CID 6575. nih.gov: NIH; 2004.

(https://pubchem.ncbi.nlm.nih.gov/compound/6575\#section=Top). (Accessed July 2017). 
115. Registry AfTSaD. Toxicological Profile for Trichloroethylene. atsdr.cdc.gov: CDC; 2015.

(https://www.atsdr.cdc.gov/PHS/PHS.asp?id=171\&tid=30). (Accessed July 2017).

116. Agency USEP. EPA Health Effects Notebook for Hazardous Air Pollutants: Trichloroethylene. epa.gov: EPA; 2000.

(https://www.epa.gov/sites/production/files/2016-

09/documents/trichloroethylene.pdf). (Accessed July 2017).

117. Lash LH, Fisher JW, Lipscomb JC, et al. Metabolism of trichloroethylene. Environmental health perspectives 2000;108 Suppl 2:177-200.

118. Ford ES, Giles WH, Dietz WH. Prevalence of the metabolic syndrome among US adults: findings from the third National Health and Nutrition Examination Survey. Jama 2002;287(3):356-9.

119. Information NCfB. PubChem Compound Database; Xylene, CID 7237. PubChem Open Chemistry Database; 2005.

(https://pubchem.ncbi.nlm.nih.gov/compound/7237\#section=ComputedProperties). (Accessed June 2017).

120. Registry AfTSaD. Toxic Substances Portal - Xylenes. CDC.gov; 2007. (https://www.atsdr.cdc.gov/toxfaqs/tf.asp?id=295\&tid=53). (Accessed 2017).

121. Gerin M, Siemiatycki J, Desy M, et al. Associations between several sites of cancer and occupational exposure to benzene, toluene, xylene, and styrene: results of a case-control study in Montreal. American journal of industrial medicine 1998;34(2):144-56.

122. III A. Third report of the expert panel on detection, evaluation, and treatment of high blood cholesterol in adults, Adult Treatment Panel (APT) III. National Heart, Lung and Blood Institute, 2001.

123. Park ES, Symanski E, Han D, et al. Part 2. Development of Enhanced Statistical Methods for Assessing Health Effects Associated with an Unknown Number of Major Sources of Multiple Air Pollutants. Research report (Health Effects Institute) 2015(183 Pt 1-2):51-113.

124. Coull BA, Bobb JF, Wellenius GA, et al. Part 1. Statistical Learning Methods for the Effects of Multiple Air Pollution Constituents. Research report (Health Effects Institute) 2015(183 Pt 1-2):5-50.

125. Fournier K, Glorennec $\mathrm{P}$, Bonvallot N. An exposure-based framework for grouping pollutants for a cumulative risk assessment approach: case study of indoor semi-volatile organic compounds. Environmental research 2014;130:20-8. 
126. Villeneuve PJ, Jerrett M, Su J, et al. A cohort study of intra-urban variations in volatile organic compounds and mortality, Toronto, Canada. Environmental pollution 2013;183:30-9.

127. Riley EA, Schaal L, Sasakura M, et al. Correlations between short-term mobile monitoring and long-term passive sampler measurements of trafficrelated air pollution. Atmos Environ (1994) 2016;132:229-39.

128. Li W, Dorans KS, Wilker EH, et al. Ambient air pollution, adipokines, and glucose homeostasis: The Framingham Heart Study. Environment international 2017;111:14-22.

129. Chen H, Goldberg MS, Villeneuve PJ. A systematic review of the relation between long-term exposure to ambient air pollution and chronic diseases. Reviews on environmental health 2008;23(4):243-97.

130. Andersen ZJ, Kristiansen LC, Andersen KK, et al. Stroke and long-term exposure to outdoor air pollution from nitrogen dioxide: a cohort study. Stroke 2012;43(2):320-5.

131. Coogan PF, White LF, Jerrett M, et al. Air pollution and incidence of hypertension and diabetes mellitus in black women living in Los Angeles. Circulation 2012;125(6):767-72.

132. Dijkema MB, Mallant SF, Gehring U, et al. Long-term exposure to trafficrelated air pollution and type 2 diabetes prevalence in a cross-sectional screening-study in the Netherlands. Environmental health : a global access science source 2011;10:76.

133. Tsai DH, Wang JL, Chuang KJ, et al. Traffic-related air pollution and cardiovascular mortality in central Taiwan. The Science of the total environment 2010;408(8):1818-23.

134. Lybarger JA, Lee R, Vogt DP, et al. Medical costs and lost productivity from health conditions at volatile organic compound-contaminated superfund sites. Environmental research 1998;79(1):9-19.

135. Tonne C, Melly S, Mittleman M, et al. A case-control analysis of exposure to traffic and acute myocardial infarction. Environmental health perspectives 2007;115(1):53-7.

136. Henning RJ, Johnson GT, Coyle JP, et al. Acrolein Can Cause Cardiovascular Disease: A Review. Cardiovasc Toxicol 2017;17(3):22736.

137. Burcham PC. Acrolein and Human Disease: Untangling the Knotty Exposure Scenarios Accompanying Several Diverse Disorders. Chem Res Toxicol 2017;30(1):145-61. 
138. Galligan JJ, Smathers RL, Fritz KS, et al. Protein carbonylation in a murine model for early alcoholic liver disease. Chem Res Toxicol 2012;25(5):1012-21.

139. Gist GL, Burg JR. Trichloroethylene--a review of the literature from a health effects perspective. Toxicology and industrial health 1995;11(3):253-307.

140. Yauck JS, Malloy ME, Blair K, et al. Proximity of residence to trichloroethylene-emitting sites and increased risk of offspring congenital heart defects among older women. Birth defects research Part A, Clinical and molecular teratology 2004;70(10):808-14.

141. Gennaro V, Ceppi M, Crosignani $P$, et al. Reanalysis of updated mortality among vinyl and polyvinyl chloride workers: Confirmation of historical evidence and new findings. BMC public health 2008;8:21.

142. Laplanche A, Clavel-Chapelon F, Contassot JC, et al. Exposure to vinyl chloride monomer: results of a cohort study after a seven year follow up. The French VCM Group. Br J Ind Med 1992;49(2):134-7.

143. Cave M, Falkner KC, Ray M, et al. Toxicant-associated steatohepatitis in vinyl chloride workers. Hepatology 2010;51(2):474-81.

144. Wiwanitkit V. Benzene exposure and hypertension: an observation. Cardiovasc J Afr 2007;18(4):264-5.

145. Kotseva K, Popov T. Study of the cardiovascular effects of occupational exposure to organic solvents. International archives of occupational and environmental health 1998;71 Suppl:S87-91.

146. Chen JL, Fayerweather WE, Pell S. Mortality study of workers exposed to dimethylformamide and/or acrylonitrile. Journal of occupational medicine : official publication of the Industrial Medical Association 1988;30(10):81921.

147. Taccola A CG, Baruffini A Cardiotoxicity of dimethylformamide (DMF). Electrocardiographic findings and continuous electrocardiographic monitoring (Holter). . Giornale Italiano di Medicina del Lavoro 1981;3:149_ 51.

148. Kang-De C H-LZ. Observation on the effects of dimethylformamide on human health. Abstracts of the 9th International Congress on Occupational Health in the Chemical Industry 1981.

149. Cirla AM, Pisati G, Invernizzi E, et al. Epidemiological study on workers exposed to low dimethylformamide concentrations. G Ital Med Lav 1984;6(3-4):149-56. 
150. Fiorito A LF, Molinari S, Zanin T Liver function alterations in synthetic leather workers exposed to dimethyl formamide. . American journal of industrial medicine 1997;32:255-60.

151. Davidson JC. Cyanide, cassava, and diabetes. Lancet 1979;2(8143):635.

152. Dhas PK, Chitra P, Jayakumar S, et al. Study of the effects of hydrogen cyanide exposure in Cassava workers. Indian J Occup Environ Med $2011 ; 15(3): 133-6$.

153. Vick JA, Froehlich HL. Studies of cyanide poisoning. Archives internationales de pharmacodynamie et de therapie 1985;273(2):314-22.

154. Sulsky SI, Hooven FH, Burch MT, et al. Critical review of the epidemiological literature on the potential cardiovascular effects of occupational carbon disulfide exposure. International archives of occupational and environmental health 2002;75(6):365-80.

155. Attinger E. [Chronic carbondisulfide poisoning simulating severe vascular disease]. Schweiz Med Wochenschr 1952;82(33):829-30.

156. Jhun HJ, Lee SY, Yim SH, et al. Metabolic syndrome in carbon disulfidepoisoned subjects in Korea: does chemical poisoning induce metabolic syndrome? International archives of occupational and environmental health 2009;82(7):827-32.

157. Penn A, Snyder CA. 1,3-Butadiene exposure and cardiovascular disease. Mutation research 2007;621(1-2):42-9.

158. Matanoski GM, Santos-Burgoa C, Schwartz L. Mortality of a cohort of workers in the styrene-butadiene polymer manufacturing industry (19431982). Environmental health perspectives 1990;86:107-17.

159. Penn A, Snyder CA. Inhalation of sidestream cigarette smoke accelerates development of arteriosclerotic plaques. Circulation 1993;88(4 Pt 1):18205 .

160. Yang Z, Knight CA, Mamerow MM, et al. Prenatal environmental tobacco smoke exposure promotes adult atherogenesis and mitochondrial damage in apolipoprotein E-/- mice fed a chow diet. Circulation 2004;110(24):371520.

161. Warren GW, Alberg AJ, Kraft AS, et al. The 2014 Surgeon General's report: "The health consequences of smoking--50 years of progress": a paradigm shift in cancer care. Cancer 2014;120(13):1914-6.

162. Perez CM, Ortiz AP, Fuentes-Mattei E, et al. High prevalence of cardiometabolic risk factors in Hispanic adolescents: correlations with 
adipocytokines and markers of inflammation. Journal of immigrant and minority health / Center for Minority Public Health 2014;16(5):865-73.

163. Papakonstantinou E, Lambadiari V, Dimitriadis G, et al. Metabolic syndrome and cardiometabolic risk factors. Curr Vasc Pharmacol 2013;11(6):858-79.

164. Ong KL, Cheung BM, Man YB, et al. Prevalence, awareness, treatment, and control of hypertension among United States adults 1999-2004. Hypertension 2007;49(1):69-75.

165. Jacobs MJ, Kleisli T, Pio JR, et al. Prevalence and control of dyslipidemia among persons with diabetes in the United States. Diabetes research and clinical practice 2005;70(3):263-9.

166. Ford ES. Prevalence of the metabolic syndrome in US populations. Endocrinol Metab Clin North Am 2004;33(2):333-50.

167. Wild S, Roglic G, Green A, et al. Global prevalence of diabetes: estimates for the year 2000 and projections for 2030. Diabetes care 2004;27(5):1047-53.

168. Setayeshgar S, Whiting SJ, Vatanparast H. Prevalence of 10-year risk of cardiovascular diseases and associated risks in canadian adults: the contribution of cardiometabolic risk assessment introduction. Int $J$ Hypertens 2013;2013:276564.

169. Daviglus ML, Talavera GA, Aviles-Santa ML, et al. Prevalence of major cardiovascular risk factors and cardiovascular diseases among Hispanic/Latino individuals of diverse backgrounds in the United States. Jama 2012;308(17):1775-84.

170. Sullivan PW, Ghushchyan V, Wyatt HR, et al. The medical cost of cardiometabolic risk factor clusters in the United States. Obesity 2007;15(12):3150-8.

171. Monnier L, Colette C, Dejager S, et al. "Mild dysglycemia" in type 2 diabetes: to be neglected or not? Journal of diabetes and its complications $2015 ; 29(3): 451-8$.

172. Nathan DM, Davidson MB, DeFronzo RA, et al. Impaired fasting glucose and impaired glucose tolerance: implications for care. Diabetes care 2007;30(3):753-9.

173. Control CfD, Prevention. Diabetes: successes and opportunities for population - based prevention and control at a glance 2011. 2011. 
174. National diabetes statistics report: Estimates of diabetes and its burden in the united states, 2014. 2014.

175. Trevino G. Consensus statement on the Worldwide Standardization of the Hemoglobin A1C Measurement: the American Diabetes Association, European Association for the Study of Diabetes, International Federation of Clinical Chemistry and Laboratory Medicine, and the International Diabetes Federation: response to the Consensus Committee. Diabetes care 2007;30(12):e141.

176. Nguyen QC, Whitsel EA, Tabor JW, et al. Blood spot-based measures of glucose homeostasis and diabetes prevalence in a nationally representative population of young US adults. Annals of epidemiology 2014;24(12):903-9 e1.

177. Bonora E, Formentini G, Calcaterra F, et al. HOMA-estimated insulin resistance is an independent predictor of cardiovascular disease in type 2 diabetic subjects: prospective data from the Verona Diabetes Complications Study. Diabetes care 2002;25(7):1135-41.

178. Havel RJ, Kane JP, Balasse EO, et al. Splanchnic metabolism of free fatty acids and production of triglycerides of very low density lipoproteins in normotriglyceridemic and hypertriglyceridemic humans. The Journal of clinical investigation 1970;49(11):2017-35.

179. Zhu S, Heymsfield SB, Toyoshima H, et al. Race-ethnicity-specific waist circumference cutoffs for identifying cardiovascular disease risk factors. The American journal of clinical nutrition 2005;81(2):409-15.

180. Alberti KG, Zimmet P, Shaw J. Metabolic syndrome--a new world-wide definition. A Consensus Statement from the International Diabetes Federation. Diabetic medicine : a journal of the British Diabetic Association 2006;23(5):469-80.

181. Larsson B, Svardsudd K, Welin L, et al. Abdominal adipose tissue distribution, obesity, and risk of cardiovascular disease and death: 13 year follow up of participants in the study of men born in 1913. British medical journal 1984;288(6428):1401-4.

182. Organization WH. Waist circumference and waist-hip ratio. Report of a WHO Expert Consultation. Geneva, Switzerland, 2008.

183. Nishida C, Ko GT, Kumanyika S. Body fat distribution and noncommunicable diseases in populations: overview of the 2008 WHO Expert Consultation on Waist Circumference and Waist-Hip Ratio. European journal of clinical nutrition 2010;64(1):2-5. 
184. Han TS, van Leer EM, Seidell JC, et al. Waist circumference action levels in the identification of cardiovascular risk factors: prevalence study in a random sample. Bmj 1995;311(7017):1401-5.

185. Zimmet PZ, Alberti KG. Introduction: Globalization and the noncommunicable disease epidemic. Obesity 2006;14(1):1-3.

186. IDF. The IDF consensus worldwide definition of the metabolic syndrome. International Diabetes Federation (IDF), 2006.

187. Chan JM, Rimm EB, Colditz GA, et al. Obesity, fat distribution, and weight gain as risk factors for clinical diabetes in men. Diabetes care 1994;17(9):961-9.

188. Lemieux S, Prud'homme D, Bouchard C, et al. A single threshold value of waist girth identifies normal-weight and overweight subjects with excess visceral adipose tissue. The American journal of clinical nutrition 1996;64(5):685-93.

189. Garcia-Donaire JA, Segura J, Sierra Adl, et al. The Meaning of Cardiometabolic Risk in Hypertensive Patients. US Endocrinology 2007;00(01):60.

190. Després J-P, Lemieux I. Abdominal obesity and metabolic syndrome. Nature 2006;444(7121):881-7.

191. Redon J, Cifkova R, Laurent S, et al. Mechanisms of hypertension in the cardiometabolic syndrome. Journal of hypertension 2009;27(3):441-51.

192. Isomaa B, Almgren $\mathrm{P}$, Tuomi $\mathrm{T}$, et al. Cardiovascular morbidity and mortality associated with the metabolic syndrome. Diabetes care $2001 ; 24(4): 683-9$.

193. Benjamin SM, Valdez R, Geiss LS, et al. Estimated number of adults with prediabetes in the US in 2000: opportunities for prevention. Diabetes care 2003;26(3):645-9.

194. Sempos C, Fulwood R, Haines C, et al. The prevalence of high blood cholesterol levels among adults in the United States. Jama $1989 ; 262(1): 45-52$.

195. Hajjar I, Kotchen TA. Trends in prevalence, awareness, treatment, and control of hypertension in the United States, 1988-2000. Jama 2003;290(2):199-206.

196. Jones CA, Francis ME, Eberhardt MS, et al. Microalbuminuria in the US population: third National Health and Nutrition Examination Survey. 
American journal of kidney diseases : the official journal of the National Kidney Foundation 2002;39(3):445-59.

197. Creamer MR, Wang TW, Babb S, et al. Tobacco Product Use and Cessation Indicators Among Adults - United States, 2018. MMWR Morbidity and mortality weekly report 2019;68(45):1013-9.

198. Park YW, Zhu S, Palaniappan L, et al. The metabolic syndrome: prevalence and associated risk factor findings in the US population from the Third National Health and Nutrition Examination Survey, 1988-1994. Archives of internal medicine 2003;163(4):427-36.

199. Touwslager RN, Gielen M, Mulder AL, et al. Genetic and environmental factors in associations between infant growth and adult cardiometabolic risk profile in twins. The American journal of clinical nutrition 2013;98(4):994-1001.

200. Kaplan RC, Bangdiwala SI, Barnhart JM, et al. Smoking among U.S. Hispanic/Latino adults: the Hispanic community health study/study of Latinos. American journal of preventive medicine 2014;46(5):496-506.

201. Nagel G, Arnold FJ, Wilhelm M, et al. Environmental tobacco smoke and cardiometabolic risk in young children: results from a survey in south-west Germany. Eur Heart J 2009;30(15):1885-93.

202. Gaziano JM, Buring JE, Breslow JL, et al. Moderate alcohol intake, increased levels of high-density lipoprotein and its subfractions, and decreased risk of myocardial infarction. The New England journal of medicine 1993;329(25):1829-34.

203. Morales LS, Lara M, Kington RS, et al. Socioeconomic, cultural, and behavioral factors affecting Hispanic health outcomes. Journal of health care for the poor and underserved 2002;13(4):477-503.

204. Adams PF, Kirzinger WK, Martinez M. Summary health statistics for the U.S. population: National Health Interview Survey, 2012. Vital Health Stat 102013(259):1-95.

205. Kitsis RN, Riquelme JA, Lavandero S. Heart Disease and Cancer. Circulation 2018;138(7):692-5.

206. Johnson JA, Ma C, Kanada KN, et al. Diet and nutrition in psoriasis: analysis of the National Health and Nutrition Examination Survey (NHANES) in the United States. Journal of the European Academy of Dermatology and Venereology : JEADV 2014;28(3):327-32.

207. National Health and Nutrition Examination Survey: Plan and Operations, 1999-2010. 
208. Johnson CL, Paulose-Ram R, Ogden CL, et al. National health and nutrition examination survey: analytic guidelines, 1999-2010. Vital Health Stat 2 2013(161):1-24.

209. Woteki CE, National H, Nutrition Examination S. Integrated NHANES: uses in national policy. The Journal of nutrition 2003;133(2):582S-4S.

210. Mahaffey KR, Gartside PS, Glueck CJ. Blood lead levels and dietary calcium intake in 1- to 11-year-old children: the Second National Health and Nutrition Examination Survey, 1976 to 1980. Pediatrics 1986;78(2):257-62.

211. Kuczmarski RJ, Flegal KM, Campbell SM, et al. Increasing prevalence of overweight among US adults. The National Health and Nutrition Examination Surveys, 1960 to 1991. Jama 1994;272(3):205-11.

212. Dwyer-Lindgren L, Mackenbach JP, van Lenthe FJ, et al. Diagnosed and Undiagnosed Diabetes Prevalence by County in the U.S., 1999-2012. Diabetes care 2016;39(9):1556-62.

213. Fulgoni VL, Buckley RB. The Contribution of Fortified Ready-to-Eat Cereal to Vitamin and Mineral Intake in the U.S. Population, NHANES 2007-2010. Nutrients 2015;7(6):3949-58.

214. Olives C, Myerson R, Mokdad AH, et al. Prevalence, awareness, treatment, and control of hypertension in United States counties, 20012009. PloS one 2013;8(4):e60308.

215. Vukoja M, Bokan A, Vujasinovic G, et al. The Differences in Spirometry Predictive Equations in Classifying Presence and Degree of Lung Function Impairment: Which Suit Fits the Best? Lung 2017.

216. Jain RB. Levels of selected urinary metabolites of volatile organic compounds among children aged 6-11 years. Environmental research 2015;142:461-70.

217. El-Metwally D, Chain K, Stefanak MP, et al. Urinary metabolites of volatile organic compounds of infants in the neonatal intensive care unit. Pediatric research 2018;83(6):1158-64.

218. Hendryx M, Luo J. Latent class analysis to model multiple chemical exposures among children. Environmental research 2018;160:115-20.

219. Wasserman EJ, Reilly SM, Goel R, et al. Comparison of Biomarkers of Tobacco Exposure between Premium and Discount Brand Cigarette Smokers in the NHANES 2011-2012 Special Sample. Cancer epidemiology, biomarkers \& prevention : a publication of the American 
Association for Cancer Research, cosponsored by the American Society of Preventive Oncology 2018;27(5):601-9.

220. Wei B, Alwis KU, Li Z, et al. Urinary concentrations of PAH and VOC metabolites in marijuana users. Environment international 2016;88:1-8.

221. Churchill JE, Ashley DL, Kaye WE. Recent chemical exposures and blood volatile organic compound levels in a large population-based sample. Archives of environmental health 2001;56(2):157-66.

222. Liu B, Jia C. Effects of exposure to mixed volatile organic compounds on the neurobehavioral test performance in a cross-sectional study of US adults. Int J Environ Health Res 2015;25(4):349-63.

223. Su FC, Mukherjee B, Batterman S. Trends of VOC exposures among a nationally representative sample: Analysis of the NHANES 1988 through 2004 data sets. Atmos Environ (1994) 2011;45(28):4858-67.

224. Batterman S, Su FC, Li S, et al. Personal exposure to mixtures of volatile organic compounds: modeling and further analysis of the RIOPA data. Research report (Health Effects Institute) 2014(181):3-63.

225. Symanski E, Stock TH, Tee PG, et al. Demographic, residential, and behavioral determinants of elevated exposures to benzene, toluene, ethylbenzene, and xylenes among the U.S. population: results from 19992000 NHANES. Journal of toxicology and environmental health Part $A$ 2009;72(14):915-24.

226. Lin YS, Egeghy PP, Rappaport SM. Relationships between levels of volatile organic compounds in air and blood from the general population. Journal of exposure science \& environmental epidemiology 2008;18(4):421-9.

227. Arif AA, Shah SM. Association between personal exposure to volatile organic compounds and asthma among US adult population. International archives of occupational and environmental health 2007;80(8):711-9.

228. Jia C, D'Souza J, Batterman S. Distributions of personal VOC exposures: a population-based analysis. Environment international 2008;34(7):92231.

229. Su FC, Jia C, Batterman S. Extreme value analyses of VOC exposures and risks: A comparison of RIOPA and NHANES datasets. Atmos Environ (1994) 2012;62:97-106.

230. Jia C, Yu X, Masiak W. Blood/air distribution of volatile organic compounds (VOCs) in a nationally representative sample. The Science of the total environment 2012;419:225-32. 
231. Mumtaz MM, Ray M, Crowell SR, et al. Translational research to develop a human PBPK models tool kit-volatile organic compounds (VOCs). Journal of toxicology and environmental health Part A 2012;75(1):6-24.

232. Lemire S, Ashley D, Olaya $\mathrm{P}$, et al. Environmental exposure of commuters in Mexico City to volatile organic compounds as assessed by blood concentrations, 1998. Salud Publica Mex 2004;46(1):32-8.

233. Rosner B. Fundamentals of biostatistics. 7th ed. Boston: Brooks/Cole, Cengage Learning; 2011.

234. Institute S. SAS/STAT 9.3 User's Guide: Survey Data Analysis (Book Excerpt). SAS Institute; 2011.

235. Jesus VD. Laboratory Procedure Manual: Volatile Organic Compounds (VOCs) Metabolites. In: Tobacco and Volatiles Branch DoLS, National Center for Environmental Health, ed. Laboratory Procedure Manual, 2012.

236. Volatile Organic Compounds \& Metabolites - Urine. National Center for Health Statistics; 2017. (https://wwwn.cdc.gov/Nchs/Nhanes/20112012/UVOC_G.htm). (Accessed 2018).

237. Group NCDRFC-AW. Trends in cardiometabolic risk factors in the Americas between 1980 and 2014: a pooled analysis of population-based surveys. Lancet Glob Health 2020;8(1):e123-e33.

238. Okosun IS, Liao Y, Rotimi CN, et al. Abdominal adiposity and clustering of multiple metabolic syndrome in White, Black and Hispanic americans. Annals of epidemiology 2000;10(5):263-70.

239. Haffner SM, Stern MP, Hazuda HP, et al. Upper body and centralized adiposity in Mexican Americans and non-Hispanic whites: relationship to body mass index and other behavioral and demographic variables. Int $J$ Obes 1986;10(6):493-502.

240. Bender R, Lange S. Adjusting for multiple testing--when and how? Journal of clinical epidemiology 2001;54(4):343-9.

241. Moss S. Modified Bonferroni Adjustments. 2016. (https://www.sicotests.com/psyarticle.asp?id=251). (Accessed Feb 2020).

242. Shi Q, Pavey ES, Carter RE. Bonferroni-based correction factor for multiple, correlated endpoints. Pharm Stat 2012;11(4):300-9.

243. Jewell NP. Statistics for epidemiology. Boca Raton: Chapman \& Hall/CRC; 2004. 
244. Bühlmann P, Geer SAvd. Statistics for high-dimensional data : methods, theory and applications. Heidelberg ; New York: Springer; 2011.

245. Crippa M, Guizzardi D, Muntean M, et al. Gridded emissions of air pollutants for the period 1970-2012 within EDGAR v4.3.2. Earth Syst Sci Data 2018;10(4):1987-2013.

246. McCarthy MC, Hafner HR, Chinkin LR, et al. Temporal variability of selected air toxics in the United States. Atmos Environ 2007;41(34):718094.

247. Su FC, Mukherjee B, Batterman S. Determinants of personal, indoor and outdoor VOC concentrations: an analysis of the RIOPA data. Environmental research 2013;126:192-203.

248. Sobus JR, DeWoskin RS, Tan YM, et al. Uses of NHANES Biomarker Data for Chemical Risk Assessment: Trends, Challenges, and Opportunities. Environmental health perspectives 2015;123(10):919-27.

249. DeJarnett N, Conklin DJ, Riggs DW, et al. Acrolein exposure is associated with increased cardiovascular disease risk. Journal of the American Heart Association 2014;3(4).

250. Feroe AG, Attanasio R, Scinicariello F. Acrolein metabolites, diabetes and insulin resistance. Environmental research 2016;148:1-6.

251. Kwon JW, Park HW, Kim WJ, et al. Exposure to volatile organic compounds and airway inflammation. Environmental health : a global access science source 2018;17(1):65.

252. Kim SY, Son BS, Park HJ, et al. Impact of environmental volatile organic compounds on otitis media in children: Correlation between exposure and urinary metabolites. International journal of pediatric otorhinolaryngology 2017;93:157-62.

253. Yoon $\mathrm{HI}$, Hong YC, Cho SH, et al. Exposure to volatile organic compounds and loss of pulmonary function in the elderly. Eur Respir $J$ 2010;36(6):1270-6.

254. van Welie RT, van Dijck RG, Vermeulen NP, et al. Mercapturic acids, protein adducts, and DNA adducts as biomarkers of electrophilic chemicals. Critical reviews in toxicology 1992;22(5-6):271-306.

255. DeRooij B, Commandeur J, Vermeulen N. Mercapturic acids as biomarkers of exposure to electrophilic chemicals:applications to environmental and industrial chemicals. Biomarkers 1998;3(4-5):239-303. 
256. Heinrich-Ramm R, Jakubowski M, Heinzow B, et al. Biological monitoring for exposure to volatile organic compounds (VOCs). Pure Appl Chem 2000;72(3):385-436.

257. Henderson RF, Bechtold WE, Bond JA, et al. The Use of Biological Markers in Toxicology. Critical reviews in toxicology 1989;20(2):65-82.

258. Mathias PI, B'Hymer C. A survey of liquid chromatographic-mass spectrometric analysis of mercapturic acid biomarkers in occupational and environmental exposure monitoring. J Chromatogr B Analyt Technol Biomed Life Sci 2014;964:136-45.

259. Wallace LA. Personal exposure to 25 volatile organic compounds. EPA's 1987 team study in Los Angeles, California. Toxicology and industrial health 1991;7(5-6):203-8.

260. Bein K, Leikauf GD. Acrolein - a pulmonary hazard. Molecular nutrition \& food research 2011;55(9):1342-60.

261. Dang TN, Arseneault M, Murthy V, et al. Potential role of acrolein in neurodegeneration and in Alzheimer's disease. Curr Mol Pharmacol 2010;3(2):66-78.

262. Park YS, Taniguchi N. Acrolein induces inflammatory response underlying endothelial dysfunction: a risk factor for atherosclerosis. Annals of the New York Academy of Sciences 2008;1126:185-9.

263. Daimon M, Sugiyama K, Kameda W, et al. Increased urinary levels of pentosidine, pyrraline and acrolein adduct in type 2 diabetes. Endocr $\mathrm{J}$ 2003;50(1):61-7.

264. Suzuki D, Miyata T. Carbonyl stress in the pathogenesis of diabetic nephropathy. Intern Med 1999;38(4):309-14.

265. Conklin DJ, Malovichko MV, Zeller I, et al. Biomarkers of Chronic Acrolein Inhalation Exposure in Mice: Implications for Tobacco Product-Induced Toxicity. Toxicological sciences : an official journal of the Society of Toxicology 2017;158(2):263-74.

266. O'Toole TE, Abplanalp W, Li X, et al. Acrolein decreases endothelial cell migration and insulin sensitivity through induction of let-7a. Toxicological sciences : an official journal of the Society of Toxicology 2014;140(2):27182.

267. Andre E, Campi B, Materazzi S, et al. Cigarette smoke-induced neurogenic inflammation is mediated by alpha,beta-unsaturated aldehydes and the TRPA1 receptor in rodents. The Journal of clinical investigation 2008;118(7):2574-82. 
268. Earley S. TRPA1 channels in the vasculature. British journal of pharmacology 2012;167(1):13-22.

269. Fernandes ES, Fernandes MA, Keeble JE. The functions of TRPA1 and TRPV1: moving away from sensory nerves. British journal of pharmacology 2012;166(2):510-21.

270. Pozsgai G, Bodkin JV, Graepel R, et al. Evidence for the pathophysiological relevance of TRPA1 receptors in the cardiovascular system in vivo. Cardiovascular research 2010;87(4):760-8.

271. Smani T, Gómez LJ, Regodon S, et al. TRP Channels in Angiogenesis and Other Endothelial Functions. Frontiers in physiology 2018;9:1731-.

272. Andersson K-E. TRP Channels as Lower Urinary Tract Sensory Targets. Med Sci (Basel) 2019;7(5):67.

273. Barrick S, Chopra B, Caterina M, et al. Expression and function of the cold channels in urinary bladder urothelium: TRPM8 and TRPA1. The Journal of Pain 2005;6(3):S10.

274. Earley S, Gonzales AL, Crnich R. Endothelium-dependent cerebral artery dilation mediated by TRPA1 and Ca2+-Activated K+ channels. Circ Res 2009;104(8):987-94.

275. Sinharoy P, Bratz IN, Sinha S, et al. TRPA1 and TRPV1 contribute to propofol-mediated antagonism of U46619-induced constriction in murine coronary arteries. PloS one 2017;12(6):e0180106.

276. Conklin DJ, Guo Y, Nystoriak MA, et al. TRPA1 channel contributes to myocardial ischemia-reperfusion injury. American Journal of PhysiologyHeart and Circulatory Physiology 2019;316(4):H889-H99.

277. Andrei SR, Sinharoy P, Bratz IN, et al. TRPA1 is functionally co-expressed with TRPV1 in cardiac muscle: Co-localization at z-discs, costameres and intercalated discs. Channels (Austin) 2016;10(5):395-409.

278. Conklin DJ. Acute cardiopulmonary toxicity of inhaled aldehydes: role of TRPA1. Annals of the New York Academy of Sciences 2016;1374(1):5967.

279. Mukhopadhyay I, Kulkarni A, Khairatkar-Joshi N. Blocking TRPA1 in Respiratory Disorders: Does It Hold a Promise? Pharmaceuticals (Basel, Switzerland) 2016;9(4):70.

280. Bautista DM, Jordt SE, Nikai T, et al. TRPA1 mediates the inflammatory actions of environmental irritants and proalgesic agents. Cell 2006;124(6):1269-82. 
281. Caceres Al, Brackmann M, Elia MD, et al. A sensory neuronal ion channel essential for airway inflammation and hyperreactivity in asthma.

Proceedings of the National Academy of Sciences of the United States of America 2009;106(22):9099-104.

282. Trevisan G, Benemei S, Materazzi S, et al. TRPA1 mediates trigeminal neuropathic pain in mice downstream of monocytes/macrophages and oxidative stress. Brain : a journal of neurology 2016;139(Pt 5):1361-77.

283. Achanta S, Jordt S-E. TRPA1: Acrolein meets its target. Toxicology and applied pharmacology 2017;324:45-50.

284. Russell FA, King R, Smillie SJ, et al. Calcitonin gene-related peptide: physiology and pathophysiology. Physiological reviews 2014;94(4):1099142.

285. Trevisani M, Siemens J, Materazzi S, et al. 4-Hydroxynonenal, an endogenous aldehyde, causes pain and neurogenic inflammation through activation of the irritant receptor TRPA1. Proceedings of the National Academy of Sciences 2007;104(33):13519-24.

286. Lynch J. Tobacco-product derived aldehydes: effects on circulating angiogenic cells \& implications for cardiovascular disease. University of Louisville 2020.

287. Kurhanewicz N, Ledbetter A, Farraj A, et al. TRPA1 mediates the cardiac effects of acrolein through parasympathetic dominance but also sympathetic modulation in mice. Toxicology and applied pharmacology 2018;347:104-14.

288. Conklin DJ, Haberzettl P, Jagatheesan G, et al. Role of TRPA1 in acute cardiopulmonary toxicity of inhaled acrolein. Toxicology and applied pharmacology 2017;324:61-72.

289. Kurhanewicz N, McIntosh-Kastrinsky R, Tong H, et al. TRPA1 mediates changes in heart rate variability and cardiac mechanical function in mice exposed to acrolein. Toxicology and applied pharmacology 2017;324:5160.

290. Macpherson LJ, Dubin AE, Evans MJ, et al. Noxious compounds activate TRPA1 ion channels through covalent modification of cysteines. Nature 2007;445(7127):541-5.

291. Niaz K, Mabqool F, Khan F, et al. Molecular mechanisms of action of styrene toxicity in blood plasma and liver. Environmental toxicology 2017;32(10):2256-66. 
292. Serron SC, Dwivedi N, Backes WL. Ethylbenzene induces microsomal oxygen free radical generation: antibody-directed characterization of the responsible cytochrome P450 enzymes. Toxicology and applied pharmacology 2000;164(3):305-11.

293. Taylor-Clark TE. Role of reactive oxygen species and TRP channels in the cough reflex. Cell calcium 2016;60(3):155-62.

294. Sullivan MN, Gonzales AL, Pires PW, et al. Localized TRPA1 channel $\mathrm{Ca} 2+$ signals stimulated by reactive oxygen species promote cerebral artery dilation. Science Signaling 2015;8(358):ra2.

295. Aubdool A, Kodji X, Fernandes E, et al. 190 the participation of reactive oxygen species and trpa1 in cinnamaldehyde-induced vasodilatation in the peripheral vasculature. Heart 2013;99(suppl 2):A106.

296. Wallace LA, Pellizzari ED, Hartwell TD, et al. The TEAM (Total Exposure Assessment Methodology) Study: personal exposures to toxic substances in air, drinking water, and breath of 400 residents of New Jersey, North Carolina, and North Dakota. Environmental research 1987;43(2):290-307.

297. Abplanalp WT, Wickramasinghe NS, Sithu SD, et al. Benzene Exposure Induces Insulin Resistance in Mice. Toxicological sciences : an official journal of the Society of Toxicology 2019;167(2):426-37. 


\section{APPENDIX}

Table A.1. Study Population with Cardiometabolic Syndrome Characteristics Stratified by Gender.

\begin{tabular}{|c|c|c|c|c|c|c|c|c|c|c|}
\hline & \multicolumn{3}{|c|}{$\begin{array}{l}\text { Full Cohort } \\
\qquad \mathrm{N}=811\end{array}$} & \multicolumn{3}{|c|}{$\begin{array}{c}\text { Female } \\
n=460(56.72 \%)\end{array}$} & \multicolumn{3}{|c|}{$\begin{array}{c}\text { Male } \\
\mathrm{n}=351(43.28 \%)\end{array}$} & \multirow[b]{2}{*}{$p$-value } \\
\hline & $\mathrm{n}$ & mean/\% & SE & $\mathrm{n}$ & mean/\% & SE & $\mathrm{n}$ & mean/\% & SE & \\
\hline Age (years) & 811 & 56.81 & 0.56 & 460 & 57.72 & 0.82 & 351 & 55.65 & 1.03 & 0.158 \\
\hline Race or ethnic origin (\%) & & & & & & & & & & 0.0497 \\
\hline Mexican American & 136 & 8.91 & 1.59 & 72 & 7.40 & 1.68 & 64 & 10.83 & 1.82 & \\
\hline Other Hispanic & 52 & 4.34 & 0.85 & 32 & 4.37 & 1.11 & 20 & 4.30 & 1.11 & \\
\hline Non-Hispanic White & 286 & 62.69 & 2.77 & 159 & 60.62 & 3.89 & 127 & 65.34 & 2.52 & \\
\hline Non-Hispanic Black & 261 & 17.54 & 2.14 & 150 & 19.74 & 2.88 & 111 & 14.72 & 1.98 & \\
\hline Other/Multi-Racial & 76 & 6.53 & 1.00 & 47 & 7.88 & 1.18 & 29 & 4.80 & 1.44 & \\
\hline \multicolumn{11}{|l|}{ Medical History } \\
\hline $\mathrm{BMI}(\mathrm{kg} / \mathrm{m} 2)$ & 808 & 34.07 & 0.38 & 458 & 34.73 & 0.61 & 350 & 33.23 & 0.42 & 0.0597 \\
\hline Overweight (\%) & 745 & 94.13 & 1.14 & 442 & 96.72 & 0.81 & 303 & 90.83 & 2.11 & 0.0003 \\
\hline Type 2 diabetes (\%) & 308 & 39.77 & 2.06 & 174 & 38.37 & 3.07 & 134 & 41.60 & 2.87 & 0.4629 \\
\hline $\mathrm{HbA} 1 \mathrm{c}$ & 810 & 6.53 & 0.05 & 459 & 6.53 & 0.07 & 351 & 6.52 & 0.07 & 0.8682 \\
\hline Dysglycemia (\%) & 811 & 100.00 & 0.00 & 460 & 100.00 & 0.00 & 351 & 100.00 & 0.00 & \\
\hline HDL-cholesterol & & & & & & & & & & $<.0001$ \\
\hline men & 351 & 42.40 & 0.96 & --- & --- & --- & 351 & 42.40 & 0.96 & \\
\hline Women & 460 & 51.16 & 0.80 & 460 & 51.16 & 0.80 & --- & --- & --- & \\
\hline Low HDL-cholesterol (\%) & 187 & 24.48 & 2.23 & 90 & 21.89 & 2.56 & 97 & 27.79 & 3.68 & 0.1679 \\
\hline Systolic Blood Pressure & 780 & 132.85 & 1.23 & 437 & 132.79 & 1.33 & 343 & 132.94 & 1.74 & 0.9364 \\
\hline Diastolic Blood Pressure & 775 & 73.24 & 0.74 & 433 & 71.20 & 0.87 & 342 & 75.78 & 1.31 & 0.0073 \\
\hline Hypertension (\%) & 663 & 80.93 & 2.13 & 384 & 82.13 & 2.38 & 279 & 79.42 & 3.36 & 0.4719 \\
\hline Albumin:Creatinine Ratio & 811 & 84.47 & 13.69 & 460 & 79.61 & 18.94 & 351 & 90.66 & 18.57 & 0.6721 \\
\hline Microalbuminuria (\%) & 241 & 25.92 & 2.40 & 123 & 26.70 & 3.20 & 118 & 24.93 & 3.14 & 0.6724 \\
\hline Urinary creatinine & 811 & 114.70 & 3.30 & 460 & 102.39 & 4.17 & 351 & 130.40 & 4.98 & $<.0001$ \\
\hline Cardiometacolic Syndrome (\%) & 811 & 13.80 & 0.55 & 460 & 7.74 & 0.45 & 351 & 6.07 & 0.45 & 0.0718 \\
\hline \multicolumn{11}{|l|}{ Lifestyle } \\
\hline$<$ High school Education (\%) & 252 & 20.76 & 1.86 & 142 & 19.49 & 2.30 & 110 & 22.40 & 2.46 & 0.3309 \\
\hline Below $\$ 20,000 \mathrm{HH}$ income (\%) & 194 & 18.47 & 1.71 & 120 & 22.73 & 2.32 & 74 & 13.03 & 1.74 & $<.0001$ \\
\hline Smoking exposure (\%) & & & & & & & & & & 0.0002 \\
\hline Current smoker & 183 & 24.70 & 2.10 & 79 & 18.30 & 2.27 & 104 & 32.57 & 3.27 & \\
\hline Secondhand Smoke exp & 173 & 19.32 & 2.15 & 98 & 19.99 & 2.86 & 75 & 18.49 & 2.09 & \\
\hline Non-exposed & 397 & 55.98 & 2.21 & 245 & 61.70 & 2.74 & 152 & 48.94 & 3.61 & \\
\hline Serum cotinine & 811 & 54.90 & 6.12 & 460 & 43.27 & 6.13 & 351 & 69.73 & 9.40 & 0.0063 \\
\hline Consume alcohol (\%) & 479 & 69.10 & 2.43 & 211 & 56.93 & 3.53 & 268 & 84.60 & 2.72 & $<.0001$ \\
\hline Physically inactive (\%) & 355 & 39.17 & 2.11 & 220 & 42.90 & 3.24 & 135 & 34.41 & 3.60 & 0.1196 \\
\hline
\end{tabular}

Data are mean (SE), \% (SE). All percentages are weighted to account for NHANES complex sampling design and survey weights. 


\section{Table A.2. Volatile Organic Compound Metabolite Level of Detection Stratified by Tobacco Smoke Exposure Status.}

\begin{tabular}{|c|c|c|c|c|c|}
\hline \multirow[b]{2}{*}{ Parent Compound } & \multirow[b]{2}{*}{ VOC metabolite (IUPAC name) } & \multirow[b]{2}{*}{ Metabolite } & \multirow[b]{2}{*}{$\begin{array}{c}\text { LLOD } \\
(\mathrm{ng} / \mathrm{mL})\end{array}$} & \multicolumn{2}{|c|}{ Percent At or Above LOD } \\
\hline & & & & $\begin{array}{l}\text { Non Smoke } \\
n=2110\end{array}$ & $\begin{array}{l}\text { Smoke } \\
n=2176\end{array}$ \\
\hline \multirow[t]{2}{*}{ Acrolein } & N-Acetyl-S- (2-carboxyethyl)-L-cysteine & CEMA & 6.96 & 97.49 & 99.27 \\
\hline & N-Acetyl-S- (3-Hydroxypropyl)-L-cysteine & 3HPMA & 13.0 & 99.06 & 99.56 \\
\hline \multirow[t]{2}{*}{ Acrylamide } & N-Acetyl-S-(2-carbamoylethyl)-L-cysteine & AAMA & 2.20 & 99.38 & 99.68 \\
\hline & $\begin{array}{l}\text { N-Acetyl-S-(2-carbamoyl-2-hydroxyethyl)-L- } \\
\text { cysteine }\end{array}$ & GAMA & 9.40 & 37.73 & 60.66 \\
\hline Acrylonitrile & N-Acetyl-S-(2-cyanoethyl)-L-cysteine & CYMA & 0.500 & 82.32 & 95.29 \\
\hline $\begin{array}{l}\text { Acrylonitrile, vinyl } \\
\text { chloride, ethylene oxide }\end{array}$ & N-Acetyl-S- (2-hydroxyethyl)-L-cysteine & HEMA & 0.791 & 26.87 & 50.59 \\
\hline Benzene & N-Acetyl-S-(phenyl)-L-cysteine & PMA & 0.600 & 29.45 & 35.99 \\
\hline 1-Bromopropane & N-Acetyl-S-(n-propyl)-L-cysteine & BPMA & 1.20 & 71.50 & 72.03 \\
\hline \multirow[t]{2}{*}{ 1,3-Butadiene } & N-Acetyl-S- (3,4-dihydroxybutyl)-L-cysteine & DHBMA & 5.25 & 99.79 & 99.64 \\
\hline & N-Acetyl-S-(4-hydroxy-2-buten-1-yl)-L-cysteine & MHBMA3 & 0.600 & 96.37 & 97.87 \\
\hline Carbon-disulfide & 2-Thioxothiazolidine-4-carboxylic acid & TTCA & 11.2 & 33.82 & 39.99 \\
\hline Crotonaldehyde & N-Acetyl-S-(3-hydroxypropyl-1-methyl)-L-cysteine & HPMMA & 2.00 & 99.99 & 99.94 \\
\hline Cyanide & 2-Aminothiazoline-4-carboxylic acid & ATCA & 15.0 & 84.85 & 82.58 \\
\hline$N, N$ - Dimethylformamide & N-Acetyl-S-(N-methylcarbamoyl)-L-cysteine & AMCC & 6.26 & 99.24 & 99.51 \\
\hline Ethylbenzene, styrene & Phenylglyoxylic acid & PGA & 12.0 & 91.11 & 92.48 \\
\hline Propylene oxide & N-Acetyl-S-(2-hydroxypropyl)-L-cysteine & 2HPMA & 5.30 & 91.04 & 96.52 \\
\hline \multirow[t]{2}{*}{ Styrene } & $\begin{array}{l}\text { N-Acetyl-S-(1-phenyl-2-hydroxyethyl-L-cysteine + } \\
\text { N-Acetyl-S-(2-phenyl-2-hydroxyethyl)-L-cysteine }\end{array}$ & PHEMA & 0.700 & 16.87 & 27.94 \\
\hline & Mandelic acid & MA & 12.0 & 98.29 & 98.97 \\
\hline Toluene & N-Acetyl-S-(benzyl)-L-cysteine & BMA & 0.500 & 99.09 & 98.61 \\
\hline \multirow[t]{2}{*}{ Xylene } & 2-Methylhippuric acid & $2 \mathrm{MHA}$ & 5.00 & 91.47 & 96.84 \\
\hline & 3-Methylhippuric acid + 4-Methylhippuric acid & $3 \& 4 \mathrm{MHA}$ & 8.00 & 99.39 & 99.84 \\
\hline
\end{tabular}


Table A.3. Description of Volatile Organic Compound Metabolites by NHANES Cycle.

\begin{tabular}{|c|c|c|c|c|c|c|c|c|c|c|c|c|c|c|c|c|c|c|c|c|c|c|c|}
\hline \multirow[b]{2}{*}{ Parent Compound } & \multicolumn{9}{|c|}{ 2005/06 ( $n=1784)$} & \multicolumn{5}{|c|}{ 2011/12 ( $n=1380)$} & \multicolumn{9}{|c|}{ 2013/14 (n=1490) } \\
\hline & Metabolite & GM & SE & Min & Q1 & Median & Q3 & Max & GM & SE & Min & Q1 & Median & Q3 & Max & GM & SE & $\operatorname{Min}$ & Q1 & Median & Q3 & Max & $p$-Value \\
\hline \multirow[t]{2}{*}{ Acrolein } & CEMA & 88.60 & 3.26 & 1.02 & 51.66 & 85.03 & 151.42 & 2026.09 & 101.73 & 3.31 & 3.64 & 58.35 & 95.60 & 164.40 & 1538.46 & 94.16 & 4.67 & 6.00 & 55.32 & 90.15 & 155.07 & 4352.63 & 0.0250 \\
\hline & 3HPMA & 257.11 & 12.78 & 1.90 & 124.07 & 215.52 & 445.87 & 8076.92 & 265.33 & 8.57 & 6.81 & 134.60 & 211.68 & 452.11 & 6480.00 & 256.98 & 13.84 & 13.28 & 133.03 & 216.21 & 459.64 & 8782.05 & 0.8102 \\
\hline \multirow[t]{2}{*}{ Acrylamide } & AAMA & 57.91 & 1.83 & 0.32 & 34.66 & 53.56 & 96.31 & 1518.75 & 53.02 & 1.79 & 1.16 & 31.59 & 48.84 & 81.99 & 1408.16 & 54.72 & 3.13 & 3.99 & 31.28 & 51.13 & 90.90 & 922.22 & 0.1681 \\
\hline & GAMA & 14.51 & 0.39 & 1.37 & 9.20 & 14.27 & 23.71 & 118.49 & 11.75 & 0.32 & 1.53 & 7.32 & 11.46 & 17.93 & 168.57 & 11.84 & 0.43 & 1.83 & 7.34 & 11.31 & 18.78 & 165.79 & $<.0001$ \\
\hline Acrylonitrile & CYMA & 4.57 & 0.43 & 0.07 & 1.02 & 1.68 & 26.71 & 981.32 & 4.32 & 0.29 & 0.26 & 1.07 & 1.83 & 8.47 & 907.14 & 4.20 & 0.31 & 0.19 & 1.05 & 1.89 & 8.90 & 1623.08 & 0.7809 \\
\hline $\begin{array}{l}\text { Acrylonitrile, vinyl chloride, } \\
\text { ethylene oxide }\end{array}$ & HEMA & 0.89 & 0.06 & 0.10 & 0.41 & 0.75 & 1.65 & 31.50 & 1.25 & 0.05 & 0.13 & 0.62 & 1.16 & 2.23 & 42.65 & 1.11 & 0.04 & 0.12 & 0.58 & 1.03 & 1.86 & 44.63 & 0.0002 \\
\hline Benzene & PMA & 0.55 & 0.02 & 0.08 & 0.30 & 0.49 & 0.94 & 21.43 & 0.79 & 0.03 & 0.11 & 0.48 & 0.79 & 1.24 & 13.00 & 0.72 & 0.04 & 0.09 & 0.42 & 0.71 & 1.17 & 14.44 & $<.0001$ \\
\hline 1-Bromopropane & BPMA & 3.95 & 0.16 & 0.18 & 1.52 & 3.51 & 9.54 & 436.00 & 5.22 & 0.26 & 0.20 & 1.93 & 4.89 & 12.68 & 853.66 & 4.12 & 0.24 & 0.18 & 1.54 & 3.86 & 9.55 & 2288.89 & 0.0003 \\
\hline \multirow[t]{2}{*}{ 1,3-Butadiene } & DHBMA & 260.90 & 12.47 & 0.77 & 198.83 & 272.20 & 358.88 & 1908.33 & 291.92 & 6.87 & 2.75 & 225.03 & 286.81 & 379.19 & 3160.00 & 280.65 & 10.67 & 19.23 & 211.20 & 281.44 & 376.81 & 3800.00 & 0.1112 \\
\hline & MHBMA3 & 6.52 & 0.37 & 0.09 & 2.91 & 5.16 & 11.42 & 263.64 & 6.65 & 0.27 & 0.31 & 3.15 & 5.32 & 10.23 & 270.00 & 6.70 & 0.40 & 0.24 & 3.40 & 5.45 & 11.00 & 328.42 & 0.9411 \\
\hline Carbon-disulfide & TTCA & 14.33 & 0.43 & 1.43 & 6.87 & 12.77 & 24.03 & 1460.00 & 15.77 & 0.75 & 1.82 & 7.46 & 13.39 & 27.68 & 1169.01 & 16.44 & 0.51 & 1.71 & 7.45 & 14.60 & 29.12 & 2140.74 & 0.0092 \\
\hline Crotonaldehyde & HPMMA & 448.42 & 25.59 & 0.29 & 229.64 & 339.17 & 719.50 & 12188.00 & 529.32 & 17.21 & 2.11 & 275.65 & 405.51 & 729.70 & 15500.00 & 473.03 & 19.67 & 32.24 & 254.14 & 375.82 & 788.50 & 19421.00 & 0.0209 \\
\hline Cyanide & ATCA & 66.64 & 6.18 & 1.92 & 20.73 & 93.97 & 210.19 & 1828.21 & 114.96 & 4.77 & 2.44 & 59.65 & 120.90 & 220.20 & 1453.78 & 108.16 & 5.00 & 3.09 & 59.07 & 122.40 & 213.87 & 2179.41 & $<.0001$ \\
\hline $\mathrm{N}, \mathrm{N}$ - Dimethylformamide & AMCC & 152.80 & 7.36 & 0.92 & 79.55 & 153.31 & 280.14 & 2023.08 & 182.83 & 6.63 & 3.28 & 99.79 & 174.47 & 330.52 & 2507.14 & 165.41 & 8.89 & 6.78 & 87.56 & 162.83 & 299.69 & 3578.95 & 0.0150 \\
\hline Ethylbenzene, styrene & PGA & 100.46 & 4.69 & 1.76 & 51.11 & 135.79 & 223.50 & 55667.00 & 201.25 & 7.11 & 9.66 & 145.89 & 204.65 & 284.42 & 32525.00 & 194.24 & 8.88 & 6.44 & 134.91 & 191.83 & 281.52 & 3461.54 & $<.0001$ \\
\hline Propylene oxide & 2HPMA & 38.30 & 1.60 & 0.77 & 20.84 & 34.03 & 67.49 & 28130.00 & 37.94 & 1.93 & 2.74 & 19.65 & 31.84 & 60.64 & 5421.05 & 31.49 & 1.93 & 2.11 & 16.72 & 27.40 & 52.69 & 5000.00 & 0.0287 \\
\hline \multirow[t]{2}{*}{ Styrene } & PHEMA & 0.59 & 0.01 & 0.09 & 0.31 & 0.51 & 0.98 & 665.56 & 0.83 & 0.02 & 0.11 & 0.46 & 0.75 & 1.31 & 555.56 & 0.86 & 0.03 & 0.11 & 0.49 & 0.81 & 1.41 & 144.04 & $<.0001$ \\
\hline & MA & 144.56 & 3.29 & 1.76 & 99.07 & 138.20 & 207.93 & 31000.00 & 146.45 & 5.96 & 6.20 & 97.41 & 133.88 & 205.62 & 27778.00 & 133.30 & 6.68 & 4.17 & 85.73 & 129.08 & 199.87 & 3115.79 & 0.2935 \\
\hline Toluene & BMA & 6.91 & 0.15 & 0.07 & 3.96 & 6.29 & 10.58 & 7144.14 & 7.29 & 0.24 & 0.26 & 4.14 & 6.71 & 12.05 & 347.22 & 7.11 & 0.34 & 0.22 & 3.93 & 6.51 & 12.04 & 258.00 & 0.3853 \\
\hline \multirow[t]{2}{*}{ Xylene } & $2 \mathrm{MHA}$ & 36.16 & 1.65 & 0.73 & 16.43 & 33.40 & 78.06 & 1950.82 & 39.94 & 2.01 & 2.41 & 17.88 & 37.60 & 88.01 & 18384.00 & 35.81 & 2.38 & 1.33 & 15.98 & 36.39 & 78.57 & 1458.97 & 0.2723 \\
\hline & 3\&4MHA & 266.40 & 10.84 & 1.17 & 123.39 & 227.48 & 551.60 & 102459.00 & 259.29 & 10.98 & 9.12 & 109.29 & 217.98 & 613.56 & 55556.00 & 246.49 & 13.64 & 5.33 & 106.25 & 227.51 & 591.84 & 12456.00 & 0.5323 \\
\hline
\end{tabular}

$\mathrm{GM}$ - geometric mean. Values are $\mathrm{ng} / \mathrm{mg}$ creatinine 
Table A.4. Full Appended Study Group UM-VOC Correlation Matrix.

\begin{tabular}{|c|c|c|c|c|c|c|c|c|c|c|c|c|c|c|c|c|c|c|c|c|c|c|}
\hline & & CEMA & HPMA3 & AAMA & GAMA & CYMA & HEMA & PMA & BPMA & DHBMA & MHBMA3 & TTCA & HPMMA & ATCA & AMCC & PGA & HPMA2 & PHEMA & MA & BMA & MHA2 & MHA34 \\
\hline \multirow[t]{2}{*}{ Acrolein } & CEMA & 1.000 & 0.703 & 0.464 & 0.354 & 0.538 & 0.414 & 0.187 & 0.219 & 0.523 & 0.655 & 0.210 & 0.624 & 0.193 & 0.473 & 0.303 & 0.425 & 0.233 & 0.426 & 0.213 & 0.319 & 0.332 \\
\hline & HPMA3 & 0.703 & 1.000 & 0.548 & 0.410 & 0.682 & 0.485 & 0.279 & 0.314 & 0.516 & 0.798 & 0.247 & 0.798 & 0.220 & 0.549 & 0.347 & 0.501 & 0.307 & 0.521 & 0.186 & 0.436 & 0.423 \\
\hline \multirow[t]{2}{*}{ Acrylamide } & AAMA & 0.464 & 0.548 & 1.000 & 0.551 & 0.621 & 0.359 & 0.163 & 0.098 & 0.466 & 0.593 & 0.097 & 0.538 & 0.131 & 0.488 & 0.253 & 0.354 & 0.211 & 0.406 & 0.139 & 0.376 & 0.380 \\
\hline & GAMA & 0.354 & 0.410 & 0.551 & 1.000 & 0.451 & 0.504 & 0.392 & 0.144 & 0.319 & 0.434 & 0.247 & 0.429 & 0.179 & 0.410 & 0.182 & 0.331 & 0.403 & 0.350 & 0.100 & 0.329 & 0.290 \\
\hline Acrylonitrile & CYMA & 0.538 & 0.682 & 0.621 & 0.451 & 1.000 & 0.469 & 0.148 & -0.008 & 0.338 & 0.800 & 0.071 & 0.716 & 0.115 & 0.611 & 0.300 & 0.393 & 0.253 & 0.512 & 0.069 & 0.479 & 0.510 \\
\hline \multicolumn{23}{|l|}{$\begin{array}{l}\text { Acrylonitrile, vinyl } \\
\text { chloride, ethylene }\end{array}$} \\
\hline xide & & 0.414 & 0.485 & 0.359 & 0.504 & 0.469 & 1.000 & 0.504 & 0.260 & 0.285 & 0.481 & 0.295 & 0.496 & 0.339 & 0.403 & 0.237 & 0.365 & 0.520 & 0.389 & 0.116 & 0.349 & 0.283 \\
\hline Benzene & PMA & 0.187 & 0.279 & 0.163 & 0.392 & 0.148 & 0.504 & 1.000 & 0.225 & 0.226 & 0.268 & 0.365 & 0.283 & 0.294 & 0.213 & 0.170 & 0.216 & 0.583 & 0.270 & 0.117 & 0.237 & 0.107 \\
\hline 1-Bromopropane & BPMA & 0.219 & 0.314 & 0.098 & 0.144 & -0.008 & 0.260 & 0.225 & 1.000 & 0.169 & 0.120 & 0.229 & 0.100 & 0.211 & 0.015 & 0.103 & 0.304 & 0.173 & 0.098 & 0.124 & 0.081 & 0.003 \\
\hline \multirow[t]{2}{*}{ 1,3-Butadiene } & DHBMA & 0.523 & 0.516 & 0.466 & 0.319 & 0.338 & 0.285 & 0.226 & 0.169 & 1.000 & 0.540 & 0.166 & 0.579 & 0.220 & 0.482 & 0.470 & 0.340 & 0.237 & 0.443 & 0.305 & 0.280 & 0.315 \\
\hline & MHBMA3 & 0.655 & 0.798 & 0.593 & 0.434 & 0.800 & 0.481 & 0.268 & 0.120 & 0.540 & 1.000 & 0.162 & 0.840 & 0.220 & 0.614 & 0.337 & 0.449 & 0.298 & 0.549 & 0.142 & 0.456 & 0.459 \\
\hline Carbon-disulfide & TTCA & 0.210 & 0.247 & 0.097 & 0.247 & 0.071 & 0.295 & 0.365 & 0.229 & 0.166 & 0.162 & 1.000 & 0.171 & 0.228 & 0.104 & 0.118 & 0.158 & 0.311 & 0.161 & 0.109 & 0.110 & 0.032 \\
\hline Crotonaldehyde & HPMMA & 0.624 & 0.798 & 0.538 & 0.429 & 0.716 & 0.496 & 0.283 & 0.100 & 0.579 & 0.840 & 0.171 & 1.000 & 0.244 & 0.629 & 0.385 & 0.449 & 0.342 & 0.561 & 0.189 & 0.446 & 0.469 \\
\hline Cyanide & ATCA & 0.193 & 0.220 & 0.131 & 0.179 & 0.115 & 0.339 & 0.294 & 0.211 & 0.220 & 0.220 & 0.228 & 0.244 & 1.000 & 0.188 & 0.175 & 0.171 & 0.308 & 0.231 & 0.152 & 0.133 & 0.104 \\
\hline$N, N-$ & AMCC & & & & & & & & & & & & & & & & & & & & & \\
\hline \multicolumn{23}{|l|}{ Dimethylformamid } \\
\hline e & & 0.473 & 0.549 & 0.488 & 0.410 & 0.611 & 0.403 & 0.213 & 0.015 & 0.482 & 0.614 & 0.104 & 0.629 & 0.188 & 1.000 & 0.437 & 0.371 & 0.250 & 0.481 & 0.132 & 0.455 & 0.484 \\
\hline $\begin{array}{l}\text { Ethylbenzene, } \\
\text { styrene }\end{array}$ & PGA & 0.303 & 0.347 & 0.253 & 0.182 & 0.300 & 0.237 & 0.170 & 0.103 & 0.470 & 0.337 & 0.118 & 0.385 & 0.175 & 0.437 & 1.000 & 0.215 & 0.230 & 0.407 & 0.174 & 0.281 & 0.314 \\
\hline Propylene oxide & HPMA2 & 0.425 & 0.501 & 0.354 & 0.331 & 0.393 & 0.365 & 0.216 & 0.304 & 0.340 & 0.449 & 0.158 & 0.449 & 0.171 & 0.371 & 0.215 & 1.000 & 0.203 & 0.329 & 0.144 & 0.275 & 0.286 \\
\hline \multirow[t]{2}{*}{ Styrene } & PHEMA & 0.233 & 0.307 & 0.211 & 0.403 & 0.253 & 0.520 & 0.583 & 0.173 & 0.237 & 0.298 & 0.311 & 0.342 & 0.308 & 0.250 & 0.230 & 0.203 & 1.000 & 0.367 & 0.160 & 0.277 & 0.174 \\
\hline & MA & 0.426 & 0.521 & 0.406 & 0.350 & 0.512 & 0.389 & 0.270 & 0.098 & 0.443 & 0.549 & 0.161 & 0.561 & 0.231 & 0.481 & 0.407 & 0.329 & 0.367 & 1.000 & 0.208 & 0.444 & 0.456 \\
\hline Toluene & BMA & 0.213 & 0.186 & 0.139 & 0.100 & 0.069 & 0.116 & 0.117 & 0.124 & 0.305 & 0.142 & 0.109 & 0.189 & 0.152 & 0.132 & 0.174 & 0.144 & 0.160 & 0.208 & 1.000 & 0.104 & 0.129 \\
\hline \multirow[t]{2}{*}{ Xylene } & MHA2 & 0.319 & 0.436 & 0.376 & 0.329 & 0.479 & 0.349 & 0.237 & 0.081 & 0.280 & 0.456 & 0.110 & 0.446 & 0.133 & 0.455 & 0.281 & 0.275 & 0.277 & 0.444 & 0.104 & 1.000 & 0.841 \\
\hline & $3 \& 4 \mathrm{MHA}$ & 0.332 & 0.423 & 0.380 & 0.290 & 0.510 & 0.283 & 0.107 & 0.003 & 0.315 & 0.459 & 0.032 & 0.469 & 0.104 & 0.484 & 0.314 & 0.286 & 0.174 & 0.456 & 0.129 & 0.841 & 1.000 \\
\hline
\end{tabular}

Correlation coefficients of creatinine normalized log-transformed urinary metabolites of volatile organic compounds. 


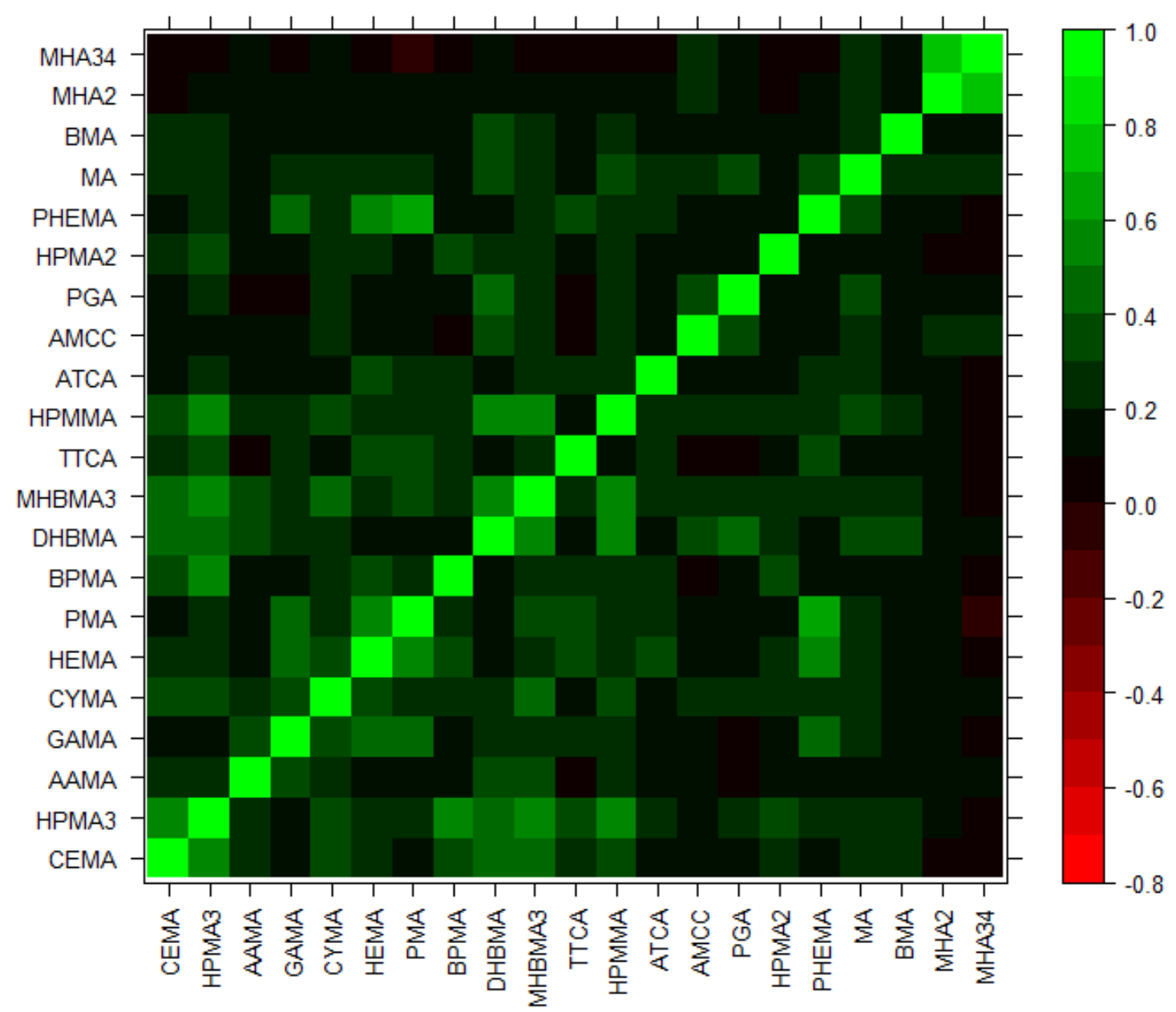

Figure A.1. Correlations of each UM-VOC with each UM-VOC- non-tobacco smoke exposed cohort. 


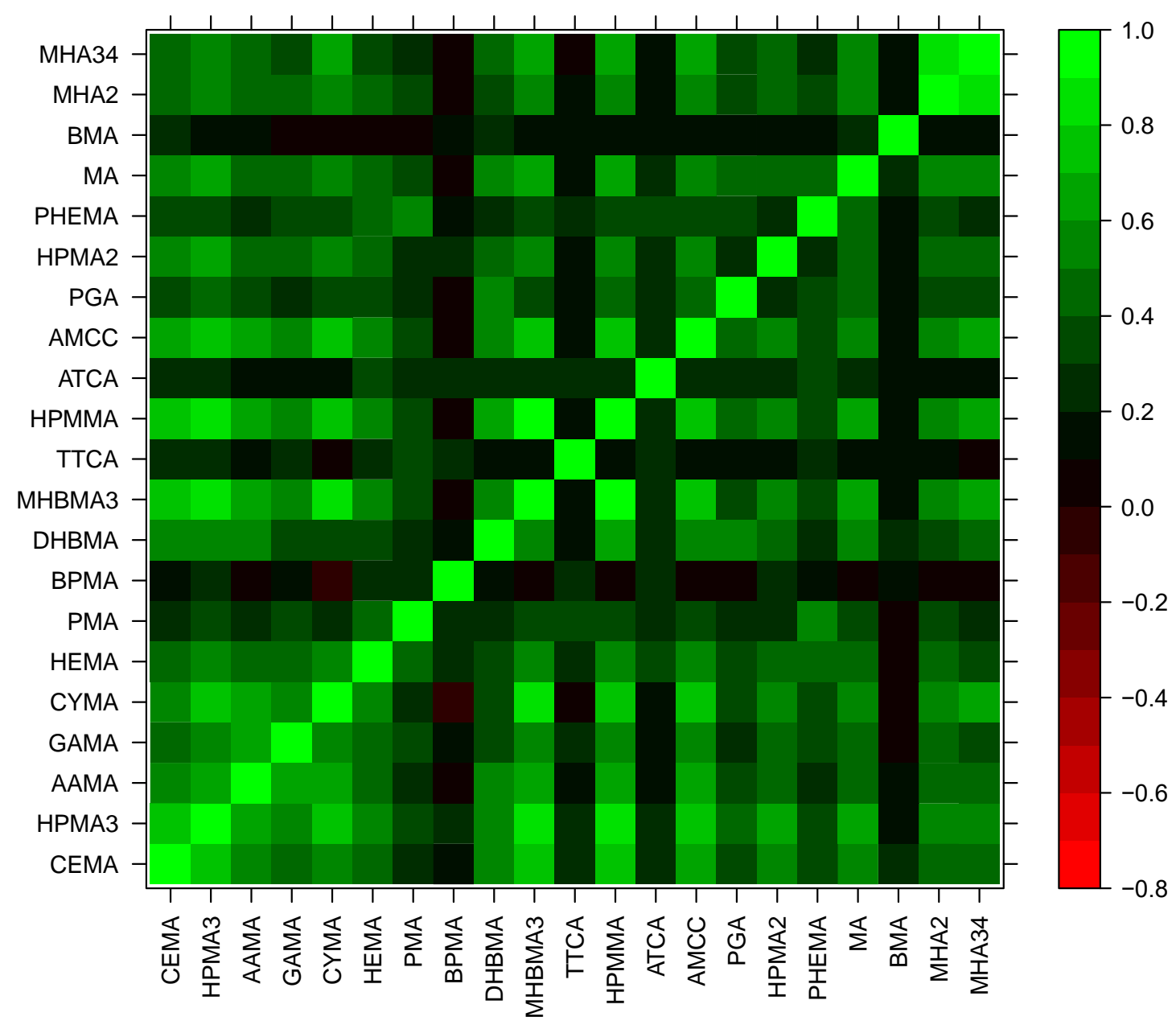

Figure A.2. Correlations of each UM-VOC with each UM-VOC- tobacco smoke exposed cohort. 
Table A.5. Unadjusted and adjusted odds of prevalent cardiometabolic disease associated with 21 urinary metabolites of volatile organic compound concentrations in NHANES participants 2005-06, 2011-12, and 2013-14.

\begin{tabular}{|c|c|c|c|c|c|c|c|c|c|}
\hline \multirow[b]{2}{*}{ Parent Compound } & \multirow[b]{2}{*}{ Metabolite } & \multicolumn{4}{|c|}{ Unadjusted Models } & \multicolumn{4}{|c|}{ Adjusted Models } \\
\hline & & $\begin{array}{l}\text { Odds } \\
\text { Ratio }\end{array}$ & $\begin{array}{l}\text { Lower } \\
\text { Cl }\end{array}$ & $\begin{array}{l}\text { Upper } \\
\text { Cl }\end{array}$ & $p$-value & $\begin{array}{l}\text { Odds } \\
\text { Ratio }\end{array}$ & $\begin{array}{c}\text { Lower } \\
\mathrm{Cl}\end{array}$ & $\begin{array}{l}\text { Upper } \\
\text { CI }\end{array}$ & p-value \\
\hline \multirow[t]{2}{*}{ Acrolein } & CEMA & 1.35 & 1.19 & 1.54 & $<.0001$ & 1.24 & 1.08 & 1.43 & 0.0032 \\
\hline & 3НРMA & 1.00 & 0.91 & 1.11 & 0.9516 & 1.06 & 0.96 & 1.18 & 0.2561 \\
\hline \multirow[t]{2}{*}{ Acrylamide } & AAMA & 0.87 & 0.78 & 0.98 & 0.0254 & 0.94 & 0.83 & 1.07 & 0.3442 \\
\hline & GAMA & 0.94 & 0.83 & 1.08 & 0.3824 & 0.94 & 0.80 & 1.09 & 0.3951 \\
\hline Acrylonitrile & CYMA & 0.94 & 0.90 & 0.99 & 0.0186 & 1.00 & 0.95 & 1.06 & 0.8718 \\
\hline $\begin{array}{l}\text { Acrylonitrile, vinyl } \\
\text { chloride, ethylene oxide }\end{array}$ & HEMA & 0.90 & 0.81 & 0.99 & 0.0364 & 0.87 & 0.77 & 0.98 & 0.0275 \\
\hline Benzene & PMA & 0.99 & 0.87 & 1.13 & 0.8873 & 0.87 & 0.74 & 1.02 & 0.0788 \\
\hline 1-Bromopropane & BPMA & 1.02 & 0.95 & 1.09 & 0.6489 & 1.02 & 0.95 & 1.10 & 0.5377 \\
\hline \multirow[t]{2}{*}{ 1,3-Butadiene } & DHBMA & 1.44 & 1.15 & 1.81 & 0.0017 & 1.13 & 0.89 & 1.42 & 0.3093 \\
\hline & МНВMАЗ & 1.02 & 0.94 & 1.11 & 0.6248 & 1.04 & 0.94 & 1.15 & 0.4439 \\
\hline Carbon-disulfide & TTCA & 1.08 & 0.98 & 1.19 & 0.1218 & 1.01 & 0.90 & 1.12 & 0.9229 \\
\hline Crotonaldehyde & HPMMA & 1.09 & 1.00 & 1.19 & 0.0533 & 1.06 & 0.96 & 1.17 & 0.2753 \\
\hline Cyanide & ATCA & 1.13 & 1.03 & 1.24 & 0.0084 & 1.03 & 0.93 & 1.15 & 0.5599 \\
\hline $\begin{array}{l}\mathrm{N}, \mathrm{N} \text { - } \\
\text { Dimethylformamide }\end{array}$ & AMCC & 1.05 & 0.96 & 1.15 & 0.2551 & 0.96 & 0.86 & 1.06 & 0.3856 \\
\hline Ethylbenzene, styrene & PGA & 1.24 & 1.11 & 1.37 & $<.0001$ & 1.20 & 1.06 & 1.36 & 0.0048 \\
\hline Propylene oxide & 2HPMA & 0.98 & 0.87 & 1.10 & 0.6935 & 0.99 & 0.89 & 1.11 & 0.8590 \\
\hline \multirow[t]{2}{*}{ Styrene } & PHEMA & 0.98 & 0.87 & 1.10 & 0.7130 & 0.83 & 0.71 & 0.97 & 0.0222 \\
\hline & $\mathrm{MA}$ & 1.02 & 0.87 & 1.18 & 0.8357 & 1.03 & 0.88 & 1.21 & 0.6962 \\
\hline Toluene & BMA & 1.02 & 0.94 & 1.12 & 0.6148 & 0.93 & 0.83 & 1.03 & 0.1576 \\
\hline \multirow[t]{2}{*}{ Xylene } & $2 \mathrm{MHA}$ & 0.90 & 0.81 & 1.00 & 0.0411 & 0.90 & 0.81 & 1.01 & 0.0602 \\
\hline & $3 \& 4 \mathrm{MHA}$ & 0.93 & 0.85 & 1.01 & 0.0794 & 0.91 & 0.83 & 1.00 & 0.0406 \\
\hline
\end{tabular}

Note: Adjusted for age, sex, race, the interaction between sex and race, and NHANES cohort year; odds ratio estimates and the corresponding $95 \%$ Wald confidence intervals $(\mathrm{Cl})$ are shown for the ordinal model 
Table A.6. Unadjusted tobacco smoke exposed and non-tobacco smoke exposed odds of prevalent cardiometabolic disease associated with 21 urinary metabolites of volatile organic compound concentrations in NHANES participants 2005-06, 2011-12, and 2013-14.

\begin{tabular}{|c|c|c|c|c|c|c|c|c|c|}
\hline \multirow[b]{2}{*}{ Parent Compound } & \multirow[b]{2}{*}{ Metabolite } & \multicolumn{4}{|c|}{$\begin{array}{c}\text { Unadjusted Tobacco Smoke } \\
\text { Exposed Models }\end{array}$} & \multicolumn{4}{|c|}{$\begin{array}{l}\text { Unadjusted Non-Tobacco } \\
\text { Smoke Exposed Models }\end{array}$} \\
\hline & & $\begin{array}{l}\text { Odds } \\
\text { Ratio }\end{array}$ & $\begin{array}{l}\text { Lower } \\
\mathrm{Cl}\end{array}$ & $\begin{array}{l}\text { Upper } \\
\text { CI }\end{array}$ & $p$-value & $\begin{array}{l}\text { Odds } \\
\text { Ratio }\end{array}$ & $\begin{array}{l}\text { Lower } \\
\text { Cl }\end{array}$ & $\begin{array}{l}\text { Upper } \\
\text { Cl }\end{array}$ & p-value \\
\hline \multirow[t]{2}{*}{ Acrolein } & CEMA & 1.26 & 1.03 & 1.53 & 0.0226 & 1.65 & 1.26 & 2.16 & 0.0005 \\
\hline & 3HPMA & 1.00 & 0.87 & 1.15 & 1.0000 & 1.12 & 0.94 & 1.34 & 0.1969 \\
\hline \multirow[t]{2}{*}{ Acrylamide } & AAMA & 0.85 & 0.73 & 0.99 & 0.0404 & 0.94 & 0.75 & 1.19 & 0.6074 \\
\hline & GAMA & 0.95 & 0.80 & 1.13 & 0.5573 & 0.95 & 0.78 & 1.15 & 0.5652 \\
\hline Acrylonitrile & CYMA & 0.95 & 0.88 & 1.02 & 0.1424 & 1.02 & 0.85 & 1.22 & 0.8667 \\
\hline $\begin{array}{l}\text { Acrylonitrile, vinyl } \\
\text { chloride, ethylene oxide }\end{array}$ & HEMA & 0.90 & 0.78 & 1.04 & 0.1656 & 0.91 & 0.79 & 1.04 & 0.1756 \\
\hline Benzene & PMA & 1.02 & 0.84 & 1.24 & 0.8338 & 0.94 & 0.79 & 1.11 & 0.4213 \\
\hline 1-Bromopropane & BPMA & 1.03 & 0.93 & 1.14 & 0.5508 & 0.99 & 0.88 & 1.12 & 0.8926 \\
\hline \multirow[t]{2}{*}{ 1,3-Butadiene } & DHBMA & 1.30 & 0.93 & 1.81 & 0.1165 & 1.71 & 1.17 & 2.48 & 0.0060 \\
\hline & MHBMA3 & 1.01 & 0.89 & 1.14 & 0.9424 & 1.22 & 1.01 & 1.47 & 0.0359 \\
\hline Carbon-disulfide & TTCA & 1.19 & 0.99 & 1.43 & 0.0597 & 1.00 & 0.84 & 1.18 & 0.9562 \\
\hline Crotonaldehyde & HPMMA & 1.05 & 0.92 & 1.19 & 0.4678 & 1.38 & 1.11 & 1.70 & 0.0045 \\
\hline Cyanide & ATCA & 1.06 & 0.94 & 1.19 & 0.3343 & 1.19 & 1.04 & 1.36 & 0.0105 \\
\hline $\begin{array}{l}N, N- \\
\text { Dimethylformamide }\end{array}$ & AMCC & 1.06 & 0.93 & 1.21 & 0.4027 & 1.15 & 0.98 & 1.34 & 0.0825 \\
\hline Ethylbenzene, styrene & PGA & 1.21 & 1.03 & 1.42 & 0.0207 & 1.36 & 1.15 & 1.60 & 0.0005 \\
\hline Propylene oxide & 2HPMA & 0.97 & 0.80 & 1.18 & 0.7374 & 1.00 & 0.87 & 1.14 & 0.9522 \\
\hline \multirow[t]{2}{*}{ Styrene } & PHEMA & 1.00 & 0.85 & 1.17 & 0.9592 & 0.94 & 0.82 & 1.08 & 0.3866 \\
\hline & MA & 1.07 & 0.87 & 1.33 & 0.5161 & 1.04 & 0.79 & 1.35 & 0.7869 \\
\hline Toluene & BMA & 0.95 & 0.82 & 1.09 & 0.4428 & 1.07 & 0.94 & 1.21 & 0.3249 \\
\hline \multirow[t]{2}{*}{ Xylene } & $2 \mathrm{MHA}$ & 0.96 & 0.83 & 1.10 & 0.5124 & 0.87 & 0.75 & 1.01 & 0.0657 \\
\hline & $3 \& 4 \mathrm{MHA}$ & 0.97 & 0.85 & 1.12 & 0.6927 & 0.92 & 0.82 & 1.04 & 0.1717 \\
\hline
\end{tabular}


Table A.7. Adjusted tobacco smoke exposed and non-tobacco smoke exposed odds of prevalent cardiometabolic disease associated with 21 urinary metabolites of volatile organic compound concentrations in NHANES participants 2005-06, 2011-12, and 2013-14.

\begin{tabular}{|c|c|c|c|c|c|c|c|c|c|}
\hline \multirow[b]{2}{*}{ Parent Compound } & \multirow[b]{2}{*}{ Metabolite } & \multicolumn{4}{|c|}{$\begin{array}{l}\text { Adjusted Tobacco Smoke } \\
\text { Exposed Models }\end{array}$} & \multicolumn{4}{|c|}{$\begin{array}{l}\text { Adjusted Non-Tobacco } \\
\text { Smoke Exposed Models }\end{array}$} \\
\hline & & $\begin{array}{l}\text { Odds } \\
\text { Ratio }\end{array}$ & $\begin{array}{l}\text { Lower } \\
\mathrm{Cl}\end{array}$ & $\begin{array}{l}\text { Upper } \\
\text { Cl }\end{array}$ & $p$-value & $\begin{array}{l}\text { Odds } \\
\text { Ratio }\end{array}$ & $\begin{array}{l}\text { Lower } \\
\mathrm{Cl}\end{array}$ & $\begin{array}{l}\text { Upper } \\
\text { Cl }\end{array}$ & p-value \\
\hline \multirow[t]{2}{*}{ Acrolein } & CEMA & 1.07 & 0.87 & 1.33 & 0.5184 & 1.38 & 1.05 & 1.80 & 0.0207 \\
\hline & 3HPMA & 1.00 & 0.85 & 1.17 & 0.9664 & 1.10 & 0.92 & 1.31 & 0.2916 \\
\hline \multirow[t]{2}{*}{ Acrylamide } & AAMA & 0.90 & 0.76 & 1.08 & 0.2556 & 0.89 & 0.70 & 1.14 & 0.3394 \\
\hline & GAMA & 0.93 & 0.75 & 1.15 & 0.5002 & 0.86 & 0.70 & 1.06 & 0.1490 \\
\hline Acrylonitrile & CYMA & 0.97 & 0.90 & 1.05 & 0.4760 & 0.94 & 0.75 & 1.18 & 0.5993 \\
\hline $\begin{array}{l}\text { Acrylonitrile, vinyl } \\
\text { chloride, ethylene oxide }\end{array}$ & HEMA & 0.86 & 0.72 & 1.02 & 0.0742 & 0.81 & 0.69 & 0.96 & 0.0171 \\
\hline Benzene & PMA & 0.89 & 0.70 & 1.13 & 0.3304 & 0.82 & 0.68 & 0.99 & 0.0403 \\
\hline 1-Bromopropane & BPMA & 1.01 & 0.89 & 1.14 & 0.8771 & 1.02 & 0.90 & 1.15 & 0.7734 \\
\hline \multirow[t]{2}{*}{ 1,3-Butadiene } & DHBMA & 1.01 & 0.76 & 1.35 & 0.9486 & 1.20 & 0.86 & 1.69 & 0.2816 \\
\hline & MHBMA3 & 0.99 & 0.85 & 1.14 & 0.8312 & 1.04 & 0.83 & 1.29 & 0.7312 \\
\hline Carbon-disulfide & TTCA & 1.12 & 0.92 & 1.37 & 0.2398 & 0.93 & 0.77 & 1.13 & 0.4763 \\
\hline Crotonaldehyde & HPMMA & 0.99 & 0.86 & 1.15 & 0.9023 & 1.11 & 0.90 & 1.38 & 0.3214 \\
\hline Cyanide & ATCA & 0.99 & 0.86 & 1.14 & 0.8779 & 1.07 & 0.91 & 1.25 & 0.4113 \\
\hline $\begin{array}{l}\mathrm{N}, \mathrm{N} \text { - } \\
\text { Dimethylformamide }\end{array}$ & AMCC & 0.94 & 0.80 & 1.11 & 0.4806 & 0.92 & 0.76 & 1.10 & 0.3313 \\
\hline Ethylbenzene, styrene & PGA & 1.16 & 0.98 & 1.38 & 0.0922 & 1.25 & 1.03 & 1.52 & 0.0272 \\
\hline Propylene oxide & 2HPMA & 0.95 & 0.77 & 1.18 & 0.6533 & 0.97 & 0.86 & 1.10 & 0.6269 \\
\hline \multirow[t]{2}{*}{ Styrene } & PHEMA & 0.83 & 0.67 & 1.03 & 0.0911 & 0.78 & 0.64 & 0.96 & 0.0178 \\
\hline & MA & 1.03 & 0.82 & 1.30 & 0.8032 & 0.97 & 0.75 & 1.27 & 0.8448 \\
\hline Toluene & BMA & 0.87 & 0.75 & 1.02 & 0.0931 & 0.95 & 0.83 & 1.09 & 0.4730 \\
\hline \multirow[t]{2}{*}{ Xylene } & $2 \mathrm{MHA}$ & 0.92 & 0.80 & 1.07 & 0.2705 & 0.84 & 0.72 & 0.98 & 0.0239 \\
\hline & $3 \& 4 \mathrm{MHA}$ & 0.94 & 0.80 & 1.09 & 0.3946 & 0.84 & 0.73 & 0.97 & 0.0182 \\
\hline
\end{tabular}

Note: Adjusted for age, sex, race, the interaction between sex and race, and NHANES cohort year; odds ratio estimates and the corresponding $95 \%$ Wald confidence intervals $(\mathrm{Cl})$ are shown for the ordinal model 
Table A.8. Full Cohort, Unadjusted odds of prevalent cardiometabolic syndrome (dichotomous) associated with 21 urinary metabolites of volatile organic compound concentrations (quintiles) in NHANES participants 2005-06, 2011-12, and 2013-14.

\begin{tabular}{|c|c|c|c|c|c|c|c|c|c|}
\hline \multirow[b]{2}{*}{ Parent Compound } & \multirow[b]{2}{*}{ Metabolite } & \multicolumn{8}{|c|}{ Appended Full Cohort $(\mathrm{N}=4654)$} \\
\hline & & $\begin{array}{l}\text { Odds } \\
\text { Ratio }\end{array}$ & $\begin{array}{l}\text { Lower } \\
\mathrm{Cl}\end{array}$ & $\begin{array}{l}\text { Upper } \\
\text { Cl }\end{array}$ & $\begin{array}{c}\text { Trend } \\
\text { p-value }\end{array}$ & $\begin{array}{l}1 \text { vs } 0 \\
\text { p-value }\end{array}$ & $\begin{array}{l}2 \text { vs } 0 \\
\text { p-value }\end{array}$ & $\begin{array}{l}3 \text { vs } 0 \\
\text { p-value }\end{array}$ & $\begin{array}{l}4 \text { vs } 0 \\
p \text {-value }\end{array}$ \\
\hline \multirow[t]{2}{*}{ Acrolein } & CEMA & 1.20 & 1.12 & 1.29 & $<.0001$ & 0.2426 & 0.0034 & 0.0004 & 0.0004 \\
\hline & 3HPMA & 1.02 & 0.95 & 1.09 & 0.5832 & 0.3533 & 0.0004 & 0.0736 & 0.7737 \\
\hline \multirow[t]{2}{*}{ Acrylamide } & AAMA & 0.93 & 0.86 & 1.00 & 0.0436 & 0.0546 & 0.6732 & 0.0244 & 0.0383 \\
\hline & GAMA & 0.96 & 0.89 & 1.03 & 0.2646 & 0.0609 & 0.9660 & 0.1835 & 0.4046 \\
\hline Acrylonitrile & CYMA & 0.94 & 0.87 & 1.01 & 0.0744 & 0.1896 & 0.1920 & 0.3229 & 0.3294 \\
\hline $\begin{array}{l}\text { Acrylonitrile, vinyl } \\
\text { chloride, ethylene oxide }\end{array}$ & HEMA & 0.94 & 0.87 & 1.01 & 0.0832 & 0.7512 & 0.4261 & 0.2764 & 0.1555 \\
\hline Benzene & PMA & 0.99 & 0.92 & 1.06 & 0.6645 & 0.7845 & 0.0568 & 0.6521 & 0.6557 \\
\hline 1-Bromopropane & BPMA & 1.01 & 0.94 & 1.08 & 0.8625 & 0.2111 & 0.7857 & 0.6536 & 0.7816 \\
\hline \multirow[t]{2}{*}{ 1,3-Butadiene } & DHBMA & 1.18 & 1.09 & 1.28 & $<.0001$ & 0.0455 & 0.0174 & 0.0042 & 0.0001 \\
\hline & МHBMA3 & 1.03 & 0.96 & 1.10 & 0.3891 & 0.0008 & 0.0235 & 0.0022 & 0.1941 \\
\hline Carbon-disulfide & TTCA & 1.03 & 0.95 & 1.12 & 0.4335 & 0.1591 & 0.7441 & 0.6264 & 0.7448 \\
\hline Crotonaldehyde & HPMMA & 1.11 & 1.03 & 1.19 & 0.0061 & 0.0194 & $<.0001$ & 0.0004 & 0.0282 \\
\hline Cyanide & ATCA & 1.08 & 1.00 & 1.17 & 0.0486 & 0.2600 & 0.0461 & 0.0845 & 0.0586 \\
\hline $\begin{array}{l}\mathrm{N}, \mathrm{N}- \\
\text { Dimethylformamide }\end{array}$ & AMCC & 1.01 & 0.94 & 1.08 & 0.7884 & 0.0688 & 0.1004 & 0.1601 & 0.3879 \\
\hline Ethylbenzene, styrene & PGA & 1.10 & 1.01 & 1.19 & 0.0265 & 0.2087 & 0.0325 & 0.0202 & 0.0583 \\
\hline Propylene oxide & 2HPMA & 0.99 & 0.90 & 1.08 & 0.7256 & 0.2126 & 0.4517 & 0.7477 & 0.8143 \\
\hline \multirow[t]{2}{*}{ Styrene } & PHEMA & 0.99 & 0.92 & 1.06 & 0.7246 & 0.3211 & 0.2821 & 0.2418 & 0.5746 \\
\hline & MA & 1.00 & 0.93 & 1.07 & 0.9868 & 0.5986 & 0.8959 & 0.6912 & 0.8777 \\
\hline Toluene & BMA & 1.01 & 0.95 & 1.08 & 0.6866 & 0.4883 & 0.8151 & 0.7410 & 0.3088 \\
\hline \multirow[t]{2}{*}{ Xylene } & $2 \mathrm{MHA}$ & 0.92 & 0.85 & 0.99 & 0.0277 & 0.6118 & 0.9915 & 0.1050 & 0.0635 \\
\hline & $3 \& 4 \mathrm{MHA}$ & 0.94 & 0.88 & 1.00 & 0.0557 & 0.7350 & 0.5142 & 0.2210 & 0.2299 \\
\hline
\end{tabular}

Note: Unadjusted odds ratio estimates and the corresponding $95 \%$ Wald confidence intervals $(\mathrm{Cl})$ are shown for the ordinal model. 
Table A.9. Full Cohort, Adjusted odds of cardiometabolic syndrome (dichotomous) associated with 21 urinary metabolites of volatile organic compound concentrations (quintiles) in NHANES participants 2005-06, 2011-12, and 2013-14.

\begin{tabular}{|c|c|c|c|c|c|c|c|c|c|}
\hline \multirow[b]{2}{*}{ Parent Compound } & \multirow[b]{2}{*}{ Metabolite } & \multicolumn{8}{|c|}{ Appended Full Cohort $(\mathrm{N}=4654)$} \\
\hline & & $\begin{array}{l}\text { Odds } \\
\text { Ratio }\end{array}$ & $\begin{array}{l}\text { Lower } \\
\text { Cl }\end{array}$ & $\begin{array}{l}\text { Upper } \\
\text { CI }\end{array}$ & $\begin{array}{l}\text { Trend } \\
\text { p-value }\end{array}$ & $\begin{array}{c}1 \text { vs } 0 \\
p-\text {-value }\end{array}$ & $\begin{array}{c}2 \text { vs } 0 \\
\text { p-value }\end{array}$ & $\begin{array}{c}3 \text { vs } 0 \\
\text { p-value }\end{array}$ & $\begin{array}{c}4 \text { vs } 0 \\
\text { p-value }\end{array}$ \\
\hline \multirow[t]{2}{*}{ Acrolein } & CEMA & 1.15 & 1.07 & 1.24 & 0.0004 & 0.3714 & 0.0158 & 0.0036 & 0.0054 \\
\hline & 3HPMA & 1.07 & 0.99 & 1.15 & 0.0793 & 0.1232 & $<.0001$ & 0.0027 & 0.1834 \\
\hline \multirow[t]{2}{*}{ Acrylamide } & AAMA & 0.97 & 0.90 & 1.05 & 0.4636 & 0.0464 & 0.9084 & 0.0595 & 0.4153 \\
\hline & GAMA & 0.96 & 0.89 & 1.04 & 0.3082 & 0.3430 & 0.4262 & 0.5170 & 0.3068 \\
\hline Acrylonitrile & CYMA & 1.00 & 0.93 & 1.09 & 0.9122 & 0.3091 & 0.3591 & 0.6702 & 0.4882 \\
\hline $\begin{array}{l}\text { Acrylonitrile, vinyl } \\
\text { chloride, ethylene oxide }\end{array}$ & HEMA & 0.92 & 0.85 & 0.99 & 0.0366 & 0.1122 & 0.2095 & 0.0436 & 0.0419 \\
\hline Benzene & PMA & 0.91 & 0.84 & 0.99 & 0.0343 & 0.2093 & 0.9645 & 0.2464 & 0.0250 \\
\hline 1-Bromopropane & BPMA & 1.01 & 0.94 & 1.09 & 0.7577 & 0.0961 & 0.8884 & 0.2910 & 0.9265 \\
\hline \multirow[t]{2}{*}{ 1,3-Butadiene } & DHBMA & 1.07 & 0.98 & 1.17 & 0.1483 & 0.0821 & 0.1402 & 0.1568 & 0.0444 \\
\hline & MHBMA3 & 1.04 & 0.96 & 1.12 & 0.3711 & 0.0202 & 0.3155 & 0.0841 & 0.1520 \\
\hline Carbon-disulfide & TTCA & 0.97 & 0.89 & 1.06 & 0.4879 & 0.0171 & 0.0954 & 0.0292 & 0.2797 \\
\hline Crotonaldehyde & HPMMA & 1.06 & 0.98 & 1.15 & 0.1413 & 0.1151 & 0.0068 & 0.0531 & 0.1203 \\
\hline Cyanide & ATCA & 1.00 & 0.92 & 1.10 & 0.9315 & 0.3268 & 0.2872 & 0.3770 & 0.8534 \\
\hline $\begin{array}{l}N, N- \\
\text { Dimethylformamide }\end{array}$ & AMCC & 0.94 & 0.87 & 1.02 & 0.1301 & 0.1842 & 0.6730 & 0.8971 & 0.6436 \\
\hline Ethylbenzene, styrene & PGA & 1.06 & 0.97 & 1.15 & 0.2212 & 0.0422 & 0.0088 & 0.0645 & 0.1310 \\
\hline Propylene oxide & 2HPMA & 1.01 & 0.93 & 1.10 & 0.8619 & 0.2300 & 0.4362 & 0.6456 & 0.6142 \\
\hline \multirow[t]{2}{*}{ Styrene } & PHEMA & 0.89 & 0.81 & 0.98 & 0.0146 & 0.6824 & 0.2702 & 0.2993 & 0.0151 \\
\hline & $\mathrm{MA}$ & 1.01 & 0.94 & 1.09 & 0.7226 & 0.7601 & 0.8491 & 0.9981 & 0.8519 \\
\hline Toluene & BMA & 0.95 & 0.88 & 1.02 & 0.1637 & 0.6318 & 0.5142 & 0.2043 & 0.4320 \\
\hline \multirow[t]{2}{*}{ Xylene } & $2 \mathrm{MHA}$ & 0.92 & 0.85 & 0.99 & 0.0301 & 0.8788 & 0.8719 & 0.2374 & 0.0612 \\
\hline & $3 \& 4 \mathrm{MHA}$ & 0.92 & 0.86 & 0.99 & 0.0252 & 0.7750 & 0.7293 & 0.2695 & 0.0526 \\
\hline
\end{tabular}

Note: Adjusted for age, sex, race, the interaction between sex and race, and NHANES cohort year; odds ratio estimates and the corresponding $95 \%$ Wald confidence intervals $(\mathrm{Cl})$ are shown for the ordinal model. 
Table A.10. Non-tobacco smoke exposed, Adjusted odds of prevalent cardiometabolic syndrome associated with 21 urinary metabolites of volatile organic compound concentrations in NHANES participants 2005-06, 2011-12, and 2013-14.

\begin{tabular}{|c|c|c|c|c|c|c|c|c|c|}
\hline \multirow[b]{2}{*}{ Parent Compound } & \multirow[b]{2}{*}{ Metabolite } & \multicolumn{8}{|c|}{ Non-Smoke Exposed $(\mathrm{N}=2074)$} \\
\hline & & $\begin{array}{l}\text { Odds } \\
\text { Ratio }\end{array}$ & $\begin{array}{l}\text { Lower } \\
\text { Cl }\end{array}$ & $\begin{array}{l}\text { Upper } \\
\text { Cl }\end{array}$ & $\begin{array}{l}\text { Trend } \\
\text { p-value }\end{array}$ & $\begin{array}{c}1 \text { vs } 0 \\
p-\text {-value }\end{array}$ & $\begin{array}{c}2 \text { vs } 0 \\
\text { p-value }\end{array}$ & $\begin{array}{c}3 \text { vs } 0 \\
p-v a l u e\end{array}$ & $\begin{array}{c}4 \text { vs } 0 \\
\text { p-value }\end{array}$ \\
\hline \multirow[t]{2}{*}{ Acrolein } & CEMA & 1.23 & 1.08 & 1.39 & 0.0025 & 0.4078 & 0.0220 & 0.0186 & 0.0084 \\
\hline & 3HPMA & 1.08 & 0.98 & 1.19 & 0.1177 & 0.1713 & 0.0004 & 0.2815 & 0.5801 \\
\hline \multirow[t]{2}{*}{ Acrylamide } & AAMA & 0.94 & 0.82 & 1.08 & 0.3645 & 0.0053 & 0.2376 & 0.0599 & 0.5225 \\
\hline & GAMA & 0.92 & 0.82 & 1.04 & 0.1670 & 0.4715 & 0.1395 & 0.6096 & 0.1354 \\
\hline Acrylonitrile & CYMA & 0.93 & 0.79 & 1.09 & 0.3382 & 0.4047 & 0.6143 & 0.1886 & 0.8769 \\
\hline $\begin{array}{l}\text { Acrylonitrile, vinyl } \\
\text { chloride, ethylene oxide }\end{array}$ & HEMA & 0.89 & 0.80 & 0.99 & 0.0377 & 0.3153 & 0.4474 & 0.1291 & 0.0457 \\
\hline Benzene & PMA & 0.91 & 0.82 & 1.01 & 0.0638 & 0.9807 & 0.9010 & 0.7167 & 0.0989 \\
\hline 1-Bromopropane & BPMA & 1.01 & 0.89 & 1.15 & 0.8642 & 0.2629 & 0.9021 & 0.2166 & 0.8308 \\
\hline \multirow[t]{2}{*}{ 1,3-Butadiene } & DHBMA & 1.09 & 0.95 & 1.25 & 0.2291 & 0.8703 & 0.3063 & 0.7593 & 0.1663 \\
\hline & MHBMA3 & 1.00 & 0.88 & 1.13 & 0.9467 & 0.3562 & 0.6709 & 0.8260 & 0.9577 \\
\hline Carbon-disulfide & TTCA & 0.93 & 0.80 & 1.07 & 0.2971 & 0.0517 & 0.0083 & 0.0605 & 0.1702 \\
\hline Crotonaldehyde & HPMMA & 1.09 & 0.96 & 1.24 & 0.1777 & 0.3779 & 0.0184 & 0.2172 & 0.3234 \\
\hline Cyanide & ATCA & 1.06 & 0.93 & 1.20 & 0.3779 & 0.4095 & 0.7193 & 0.5495 & 0.7332 \\
\hline $\begin{array}{l}N, N- \\
\text { Dimethylformamide }\end{array}$ & AMCC & 0.89 & 0.80 & 0.99 & 0.0344 & 0.7000 & 0.5679 & 0.4762 & 0.0775 \\
\hline Ethylbenzene, styrene & PGA & 1.03 & 0.90 & 1.18 & 0.6474 & 0.0317 & 0.0519 & 0.2643 & 0.3652 \\
\hline Propylene oxide & 2HPMA & 1.00 & 0.91 & 1.09 & 0.9128 & 0.0506 & 0.2560 & 0.2991 & 0.9477 \\
\hline \multirow[t]{2}{*}{ Styrene } & PHEMA & 0.88 & 0.78 & 0.99 & 0.0303 & 0.5646 & 0.7826 & 0.5683 & 0.1004 \\
\hline & $\mathrm{MA}$ & 1.00 & 0.90 & 1.10 & 0.9186 & 0.3728 & 0.5557 & 0.5080 & 0.9609 \\
\hline Toluene & BMA & 0.98 & 0.88 & 1.09 & 0.6758 & 0.7842 & 0.7350 & 0.4570 & 0.9949 \\
\hline \multirow[t]{2}{*}{ Xylene } & $2 \mathrm{MHA}$ & 0.87 & 0.77 & 0.98 & 0.0218 & 0.7629 & 0.2757 & 0.1112 & 0.0513 \\
\hline & $3 \& 4 \mathrm{MHA}$ & 0.88 & 0.79 & 0.97 & 0.0120 & 0.8096 & 0.9942 & 0.0880 & 0.0899 \\
\hline
\end{tabular}

Note: Adjusted for age, sex, race, the interaction between sex and race, and NHANES cohort year; odds ratio estimates and the corresponding 95\% Wald confidence intervals $(\mathrm{Cl})$ are shown for the ordinal model. 
Table A.11. Tobacco smoke exposed, Adjusted odds of prevalent cardiometabolic disease associated with 21 urinary metabolites of volatile organic compound concentrations in NHANES participants 2005-06, 2011-12, and 2013-14.

\begin{tabular}{|c|c|c|c|c|c|c|c|c|c|}
\hline \multirow[b]{2}{*}{ Parent Compound } & \multirow[b]{2}{*}{ Metabolite } & \multicolumn{8}{|c|}{ Smoke Exposed $(\mathrm{N}=2134)$} \\
\hline & & $\begin{array}{l}\text { Odds } \\
\text { Ratio }\end{array}$ & $\begin{array}{l}\text { Lower } \\
\text { Cl }\end{array}$ & $\begin{array}{l}\text { Upper } \\
\text { CI }\end{array}$ & $\begin{array}{l}\text { Trend } \\
\text { p-value }\end{array}$ & $\begin{array}{c}1 \text { vs } 0 \\
\text { p-value }\end{array}$ & $\begin{array}{l}2 \text { vs } 0 \\
\text { p-value }\end{array}$ & $\begin{array}{l}3 \text { vs } 0 \\
\text { p-value }\end{array}$ & $\begin{array}{c}4 \text { vs } 0 \\
\text { p-value }\end{array}$ \\
\hline \multirow[t]{2}{*}{ Acrolein } & CEMA & 1.05 & 0.90 & 1.22 & 0.5292 & 0.4904 & 0.4236 & 0.6374 & 0.4351 \\
\hline & 3HPMA & 1.02 & 0.90 & 1.17 & 0.7308 & 0.7854 & 0.4504 & 0.0943 & 0.8245 \\
\hline \multirow[t]{2}{*}{ Acrylamide } & AAMA & 0.96 & 0.85 & 1.08 & 0.4466 & 0.3107 & 0.0356 & 0.6316 & 0.8240 \\
\hline & GAMA & 0.95 & 0.85 & 1.07 & 0.3996 & 0.7019 & 0.8976 & 0.8309 & 0.5205 \\
\hline Acrylonitrile & CYMA & 0.98 & 0.86 & 1.13 & 0.8177 & 0.4179 & 0.5750 & 0.7857 & 0.8660 \\
\hline $\begin{array}{l}\text { Acrylonitrile, vinyl } \\
\text { chloride, ethylene oxide }\end{array}$ & HEMA & 0.91 & 0.80 & 1.03 & 0.1382 & 0.2127 & 0.4353 & 0.1143 & 0.1101 \\
\hline Benzene & PMA & 0.92 & 0.81 & 1.05 & 0.2061 & 0.0756 & 0.8438 & 0.4036 & 0.0911 \\
\hline 1-Bromopropane & BPMA & 0.98 & 0.88 & 1.11 & 0.7814 & 0.0645 & 0.2657 & 0.4860 & 0.3854 \\
\hline \multirow[t]{2}{*}{ 1,3-Butadiene } & DHBMA & 1.06 & 0.93 & 1.19 & 0.3805 & 0.0047 & 0.1447 & 0.0110 & 0.0436 \\
\hline & MHBMA3 & 1.02 & 0.89 & 1.18 & 0.7540 & 0.0195 & 0.1911 & 0.0580 & 0.2534 \\
\hline Carbon-disulfide & TTCA & 1.01 & 0.87 & 1.18 & 0.8602 & 0.1567 & 0.8439 & 0.0722 & 0.8849 \\
\hline Crotonaldehyde & HPMMA & 1.03 & 0.90 & 1.17 & 0.7094 & 0.1709 & 0.3356 & 0.0620 & 0.5342 \\
\hline Cyanide & ATCA & 0.95 & 0.84 & 1.08 & 0.4079 & 0.0658 & 0.2579 & 0.7546 & 0.8219 \\
\hline $\begin{array}{l}N, N \text { - } \\
\text { Dimethylformamide }\end{array}$ & AMCC & 0.97 & 0.85 & 1.10 & 0.5868 & 0.0560 & 0.2333 & 0.2589 & 0.4770 \\
\hline Ethylbenzene, styrene & PGA & 1.08 & 0.94 & 1.24 & 0.2610 & 0.3660 & 0.0497 & 0.1585 & 0.2331 \\
\hline Propylene oxide & 2HPMA & 1.00 & 0.85 & 1.17 & 0.9768 & 0.0806 & 0.2939 & 0.2859 & 0.5651 \\
\hline \multirow[t]{2}{*}{ Styrene } & PHEMA & 0.88 & 0.78 & 0.99 & 0.0388 & 0.1949 & 0.2462 & 0.3165 & 0.0093 \\
\hline & MA & 1.01 & 0.89 & 1.14 & 0.9003 & 0.6781 & 0.4226 & 0.4400 & 0.7588 \\
\hline Toluene & BMA & 0.90 & 0.81 & 1.00 & 0.0464 & 0.9237 & 0.5197 & 0.1440 & 0.2024 \\
\hline \multirow[t]{2}{*}{ Xylene } & $2 \mathrm{MHA}$ & 0.94 & 0.85 & 1.04 & 0.2367 & 0.7566 & 0.1011 & 0.9655 & 0.5588 \\
\hline & $3 \& 4 \mathrm{MHA}$ & 0.95 & 0.84 & 1.08 & 0.4475 & 0.0808 & 0.7726 & 0.9293 & 0.1685 \\
\hline
\end{tabular}

Note: Adjusted for age, sex, race, the interaction between sex and race, and NHANES cohort year; odds ratio estimates and the corresponding $95 \%$ Wald confidence intervals $(\mathrm{Cl})$ are shown for the ordinal model. 
Table A.12. Full cohort, Unadjusted odds of ordinal cardiometabolic syndrome risk score associated with 21 urinary metabolites of volatile organic compound concentrations in NHANES participants 2005-06, 2011-12, and 2013-14.

\begin{tabular}{|c|c|c|c|c|c|}
\hline \multirow[b]{2}{*}{ Parent Compound } & \multirow[b]{2}{*}{ Metabolite } & \multicolumn{4}{|c|}{ Appended Full Cohort $(\mathrm{N}=4654)$} \\
\hline & & $\begin{array}{l}\text { Odds } \\
\text { Ratio }\end{array}$ & $\begin{array}{l}\text { Lower } \\
\mathrm{Cl}\end{array}$ & $\begin{array}{l}\text { Upper } \\
\text { Cl }\end{array}$ & $p$-value \\
\hline \multirow[t]{2}{*}{ Acrolein } & CEMA & 1.38 & 1.27 & 1.51 & $<.0001$ \\
\hline & 3HPMA & 1.01 & 0.94 & 1.08 & 0.7715 \\
\hline \multirow[t]{2}{*}{ Acrylamide } & AAMA & 0.92 & 0.85 & 0.99 & 0.0368 \\
\hline & GAMA & 0.98 & 0.88 & 1.09 & 0.6993 \\
\hline Acrylonitrile & CYMA & 0.96 & 0.93 & 0.99 & 0.0149 \\
\hline \multicolumn{6}{|l|}{ Acrylonitrile, vinyl } \\
\hline chloride, ethylene oxide & HEMA & 0.94 & 0.86 & 1.03 & 0.1832 \\
\hline Benzene & PMA & 0.95 & 0.86 & 1.05 & 0.2972 \\
\hline 1-Bromopropane & BPMA & 0.97 & 0.92 & 1.02 & 0.1923 \\
\hline \multirow[t]{2}{*}{ 1,3-Butadiene } & DHBMA & 1.34 & 1.14 & 1.58 & 0.0007 \\
\hline & MHBMA3 & 1.03 & 0.97 & 1.10 & 0.3304 \\
\hline Carbon-disulfide & TTCA & 1.00 & 0.93 & 1.07 & 0.9265 \\
\hline Crotonaldehyde & HPMMA & 1.08 & 1.01 & 1.16 & 0.0366 \\
\hline Cyanide & ATCA & 1.08 & 1.02 & 1.16 & 0.0179 \\
\hline $\begin{array}{l}\mathrm{N}, \mathrm{N} \text { - } \\
\text { Dimethylformamide }\end{array}$ & AMCC & 1.12 & 1.03 & 1.21 & 0.0059 \\
\hline Ethylbenzene, styrene & PGA & 1.18 & 1.11 & 1.25 & $<.0001$ \\
\hline Propylene oxide & 2HPMA & 0.94 & 0.88 & 1.01 & 0.1142 \\
\hline \multirow[t]{2}{*}{ Styrene } & PHEMA & 1.00 & 0.91 & 1.09 & 0.9320 \\
\hline & MA & 1.03 & 0.94 & 1.13 & 0.5379 \\
\hline Toluene & BMA & 1.04 & 0.96 & 1.12 & 0.3712 \\
\hline \multirow[t]{2}{*}{ Xylene } & $2 \mathrm{MHA}$ & 0.93 & 0.87 & 1.00 & 0.0649 \\
\hline & $3 \& 4 \mathrm{MHA}$ & 0.99 & 0.93 & 1.06 & 0.7798 \\
\hline
\end{tabular}

Note: Unadjusted odds ratio estimates and the corresponding 95\% Wald confidence intervals $(\mathrm{Cl})$ 
Table A.13. Adjusted odds of ordinal cardiometabolic syndrome risk score associated with 21 urinary metabolites of volatile organic compound concentrations in NHANES participants 2005-06, 2011-12, and 2013-14.

\begin{tabular}{|c|c|c|c|c|c|}
\hline \multirow[b]{2}{*}{ Parent Compound } & \multirow[b]{2}{*}{ Metabolite } & \multicolumn{4}{|c|}{ Appended Full Cohort ( $\mathrm{N}=4654)$} \\
\hline & & $\begin{array}{l}\text { Odds } \\
\text { Ratio }\end{array}$ & $\begin{array}{l}\text { Lower } \\
\text { Cl }\end{array}$ & $\begin{array}{l}\text { Upper } \\
\text { Cl }\end{array}$ & p-value \\
\hline \multirow[t]{2}{*}{ Acrolein } & CEMA & 1.27 & 1.16 & 1.39 & $<.0001$ \\
\hline & 3HPMA & 1.04 & 0.97 & 1.13 & 0.2560 \\
\hline \multirow[t]{2}{*}{ Acrylamide } & AAMA & 0.97 & 0.90 & 1.04 & 0.3726 \\
\hline & GAMA & 0.93 & 0.83 & 1.04 & 0.2130 \\
\hline Acrylonitrile & CYMA & 1.01 & 0.98 & 1.04 & 0.5123 \\
\hline \multicolumn{6}{|l|}{ Acrylonitrile, vinyl } \\
\hline chloride, ethylene oxide & HEMA & 0.89 & 0.82 & 0.98 & 0.0136 \\
\hline Benzene & PMA & 0.80 & 0.71 & 0.89 & 0.0002 \\
\hline 1-Bromopropane & BPMA & 0.96 & 0.91 & 1.00 & 0.0607 \\
\hline \multirow[t]{2}{*}{ 1,3-Butadiene } & DHBMA & 1.07 & 0.91 & 1.26 & 0.4205 \\
\hline & MHВMAЗ & 1.03 & 0.96 & 1.10 & 0.3750 \\
\hline Carbon-disulfide & TTCA & 0.90 & 0.84 & 0.96 & 0.0012 \\
\hline Crotonaldehyde & HPMMA & 1.03 & 0.96 & 1.11 & 0.3908 \\
\hline Cyanide & ATCA & 0.96 & 0.89 & 1.04 & 0.3482 \\
\hline $\begin{array}{l}\mathrm{N}, \mathrm{N} \text { - } \\
\text { Dimethylformamide }\end{array}$ & AMCC & 0.99 & 0.93 & 1.07 & 0.8646 \\
\hline Ethylbenzene, styrene & PGA & 1.17 & 1.09 & 1.25 & $<.0001$ \\
\hline Propylene oxide & 2HPMA & 0.93 & 0.87 & 1.00 & 0.0420 \\
\hline \multirow[t]{2}{*}{ Styrene } & PHEMA & 0.84 & 0.76 & 0.93 & 0.0009 \\
\hline & MA & 1.00 & 0.91 & 1.10 & 0.9549 \\
\hline Toluene & BMA & 0.94 & 0.87 & 1.01 & 0.0939 \\
\hline \multirow[t]{2}{*}{ Xylene } & $2 \mathrm{MHA}$ & 0.92 & 0.85 & 0.99 & 0.0248 \\
\hline & $3 \& 4 \mathrm{MHA}$ & 0.96 & 0.89 & 1.03 & 0.2111 \\
\hline
\end{tabular}

Note: Adjusted for age, sex, race, the interaction between sex and race, and NHANES cohort year; odds ratio estimates and the corresponding $95 \%$ Wald confidence intervals $(\mathrm{Cl})$. 
Table A.14. Tobacco Smoke Exposed- Adjusted odds of ordinal cardiometabolic syndrome risk score associated with 21 urinary metabolites of volatile organic compound concentrations in NHANES participants 2005-06, 2011-12, and 2013-14.

\begin{tabular}{|c|c|c|c|c|c|}
\hline \multirow[b]{2}{*}{ Parent Compound } & \multirow[b]{2}{*}{ Metabolite } & \multicolumn{4}{|c|}{ Smoke Exposed $(\mathrm{N}=2134)$} \\
\hline & & $\begin{array}{l}\text { Odds } \\
\text { Ratio }\end{array}$ & $\begin{array}{l}\text { Lower } \\
\text { Cl }\end{array}$ & $\begin{array}{l}\text { Upper } \\
\text { CI }\end{array}$ & p-value \\
\hline \multirow[t]{2}{*}{ Acrolein } & CEMA & 1.07 & 0.93 & 1.24 & 0.3283 \\
\hline & 3HPMA & 0.94 & 0.85 & 1.05 & 0.2857 \\
\hline \multirow[t]{2}{*}{ Acrylamide } & AAMA & 0.86 & 0.77 & 0.97 & 0.0144 \\
\hline & GAMA & 0.89 & 0.77 & 1.03 & 0.1139 \\
\hline Acrylonitrile & CYMA & 0.96 & 0.91 & 1.01 & 0.1094 \\
\hline \multicolumn{6}{|l|}{ Acrylonitrile, vinyl } \\
\hline chloride, ethylene oxide & HEMA & 0.88 & 0.79 & 0.98 & 0.0244 \\
\hline Benzene & PMA & 0.78 & 0.68 & 0.91 & 0.0014 \\
\hline 1-Bromopropane & BPMA & 0.96 & 0.88 & 1.05 & 0.3567 \\
\hline \multirow[t]{2}{*}{ 1,3-Butadiene } & DHBMA & 1.01 & 0.81 & 1.26 & 0.9075 \\
\hline & МHВMA3 & 0.96 & 0.87 & 1.05 & 0.3040 \\
\hline Carbon-disulfide & TTCA & 0.94 & 0.83 & 1.06 & 0.2949 \\
\hline Crotonaldehyde & HPMMA & 0.96 & 0.87 & 1.06 & 0.3963 \\
\hline Cyanide & ATCA & 0.90 & 0.81 & 1.00 & 0.0516 \\
\hline $\begin{array}{l}\mathrm{N}, \mathrm{N}- \\
\text { Dimethylformamide }\end{array}$ & AMCC & 0.94 & 0.84 & 1.04 & 0.2265 \\
\hline Ethylbenzene, styrene & PGA & 1.17 & 1.05 & 1.31 & 0.0047 \\
\hline Propylene oxide & 2HPMA & 0.88 & 0.78 & 0.99 & 0.0291 \\
\hline \multirow[t]{2}{*}{ Styrene } & PHEMA & 0.80 & 0.70 & 0.92 & 0.0020 \\
\hline & $\mathrm{MA}$ & 0.94 & 0.81 & 1.09 & 0.3748 \\
\hline Toluene & BMA & 0.91 & 0.81 & 1.03 & 0.1276 \\
\hline \multirow[t]{2}{*}{ Xylene } & $2 \mathrm{MHA}$ & 0.88 & 0.79 & 0.97 & 0.0093 \\
\hline & $3 \& 4 \mathrm{MHA}$ & 0.92 & 0.83 & 1.01 & 0.0838 \\
\hline
\end{tabular}

Note: Adjusted for age, sex, race, the interaction between sex and race, and NHANES cohort year; odds ratio estimates and the corresponding $95 \%$ Wald confidence intervals $(\mathrm{Cl})$. 
Table A.15. Non-Tobacco Smoke Exposed- Adjusted odds of ordinal cardiometabolic syndrome risk score associated with 21 urinary metabolites of volatile organic compound concentrations in NHANES participants 2005-06, 2011-12, and 2013-14.

\begin{tabular}{|c|c|c|c|c|c|}
\hline \multirow[b]{2}{*}{ Parent Compound } & \multirow[b]{2}{*}{ Metabolite } & \multicolumn{4}{|c|}{ Non-Smoke Exposed $(\mathrm{N}=2074)$} \\
\hline & & $\begin{array}{l}\text { Odds } \\
\text { Ratio }\end{array}$ & $\begin{array}{c}\text { Lower } \\
\mathrm{Cl}\end{array}$ & $\begin{array}{c}\text { Upper } \\
\text { Cl }\end{array}$ & p-value \\
\hline \multirow[t]{2}{*}{ Acrolein } & CEMA & 1.39 & 1.19 & 1.62 & $<.0001$ \\
\hline & 3НPMA & 1.05 & 0.92 & 1.20 & 0.4776 \\
\hline \multirow[t]{2}{*}{ Acrylamide } & AAMA & 0.89 & 0.76 & 1.05 & 0.1747 \\
\hline & GAMA & 0.85 & 0.71 & 1.01 & 0.0615 \\
\hline Acrylonitrile & CYMA & 0.83 & 0.72 & 0.95 & 0.0078 \\
\hline \multicolumn{6}{|l|}{ Acrylonitrile, vinyl } \\
\hline chloride, ethylene oxide & HEMA & 0.79 & 0.68 & 0.93 & 0.0046 \\
\hline Benzene & PMA & 0.81 & 0.70 & 0.95 & 0.0103 \\
\hline 1-Bromopropane & BPMA & 0.96 & 0.89 & 1.04 & 0.2992 \\
\hline \multirow[t]{2}{*}{ 1,3-Butadiene } & DHBMA & 1.00 & 0.82 & 1.23 & 0.9904 \\
\hline & MHВMA3 & 0.96 & 0.82 & 1.11 & 0.5684 \\
\hline Carbon-disulfide & TTCA & 0.88 & 0.81 & 0.97 & 0.0077 \\
\hline Crotonaldehyde & HPMMA & 0.94 & 0.82 & 1.08 & 0.3649 \\
\hline Cyanide & ATCA & 1.01 & 0.91 & 1.12 & 0.8693 \\
\hline $\begin{array}{l}\mathrm{N}, \mathrm{N} \text { - } \\
\text { Dimethylformamide }\end{array}$ & AMCC & 0.91 & 0.79 & 1.05 & 0.1837 \\
\hline Ethylbenzene, styrene & PGA & 1.12 & 1.02 & 1.23 & 0.0175 \\
\hline Propylene oxide & 2HPMA & 0.90 & 0.83 & 0.98 & 0.0177 \\
\hline \multirow[t]{2}{*}{ Styrene } & PHEMA & 0.85 & 0.73 & 0.98 & 0.0252 \\
\hline & $\mathrm{MA}$ & 0.95 & 0.81 & 1.11 & 0.4863 \\
\hline Toluene & BMA & 0.93 & 0.84 & 1.03 & 0.1572 \\
\hline \multirow[t]{2}{*}{ Xylene } & $2 \mathrm{MHA}$ & 0.87 & 0.77 & 0.97 & 0.0115 \\
\hline & $3 \& 4 \mathrm{MHA}$ & 0.90 & 0.81 & 1.00 & 0.0421 \\
\hline
\end{tabular}

Note: Adjusted for age, sex, race, the interaction between sex and race, and NHANES cohort year; odds ratio estimates and the corresponding $95 \%$ Wald confidence intervals $(\mathrm{Cl})$ 


\title{
CURRICULUM VITAE
}

\author{
Stacey L. Konkle
}

\section{CONTACT INFORMATION}

Email: Stacey.Konkle@Louisville.edu

Personal Email: S_Konkle@aol.com

Linkedin.com/in/Stacey-Konkle

EDUCATION

University of Louisville

May 2020

Louisville, Kentucky

Ph.D. Epidemiology

Dissertation: "Volatile Organic Compound Exposure and Cardiometabolic Syndrome Risk in a Nationally Representative Cohort"

Dissertation Committee Co-Chairs: Kristina M. Zierold, Ph.D., and Kira C. Taylor, Ph.D.

Cumulative GPA: 3.89

University of Louisville

May 2014

Louisville, Kentucky

Masters of Public Health

Cumulative GPA: 3.94

Purdue University

May 2011

West Lafayette, Indiana

B.S. Food Science

Cumulative GPA: 3.41 
University of Louisville, School of Medicine, Institute of Molecular Cardiology/Envirome Institute

Graduate Research Assistant

2015 - Present

Mentor: Aruni Bhatnagar, Ph.D.

- Conduct research focused on urinary volatile organic compound (VOC) metabolite concentrations as biomarkers of exposure and associations with cardiovascular and metabolic injury. My present work utilizes the National Health and Nutrition Examination Survey (NHANES), which is a complex multistage probability sampled survey designed to provide a nationally representative sample cohort and the National Monitoring Program (NMP) for air toxics data to establish temporal trends in associations with ambient air VOCs and actual personal biomarkers of exposure and associations with cardiometabolic health endpoints at the national level.

- Assist in the study design and implementation of an innovative and complex community-based intervention trial. This real-world public health study focuses on mitigating the effects air pollution via increasing aerial greenness.

National Institute of Environmental Health Sciences (NIEHS)- University of Louisville Superfund Research Center

\section{Superfund Research Program Fellow}

2018 - Present

Mentor: Aruni Bhatnagar, Ph.D., and Sanjay Srivastava, Ph.D.

- Conduct analysis of environmental biomarkers of exposure and cardiometabolic outcomes to establish national representative benchmarks to inform and complement the development of the University of Louisville Superfund Center projects.

- Present research findings at regional, national, and international scientific meetings.

- Work in a multidisciplinary research teams addressing the central hypothesis that exposure to environmental volatile organic compounds cause/exacerbate insulin resistance, leading to cardiometabolic disease.

- Participate in training on grant writing, presentation skills, professional development, problem solving, regulatory science, community engagement, and research translation.

National Institutes of Health- Tobacco Centers of Regulatory Science (NIH-TCORS)- American Heart Association Tobacco Regulation and Addiction Center

\section{Early Career Investigator Fellow}

Mentor: Aruni Bhatnagar, Ph.D., Glenn Hirsch, M.D., Emelia Benjamin, M.D.

- Conducted research focused on FDA tobacco-related interests of reducing toxicity, carcinogenicity, and adverse health consequences. This research focused on smoking associated urinary volatile organic compound metabolite concentrations and cardiovascular injury biomarkers.

- Presented research findings at regional and national scientific meetings. 
- Training included presentations during weekly trainee meetings, progress reports, and peer mentoring activists.

- Weekly training sessions covering various aspects of tobacco regulatory science including career development, addiction training, translating scientific research into policy, research and writing skills, responsiveness to FDA research guidelines, and submitting FDA docket comments.

\section{PROFESSIONAL EXPERIENCE}

Louisville Metro Department of Public Health and Wellness

\section{Medical Reserve Corp- Member}

2017 - Present

- Actively participate in monthly meetings and trainings; Stop the Bleed certified, HIPPA certified, Incident Command Structure (ICS) 100-800 training, survey design and interviewing skills training, data collection tools-Epi Info software, terrorism awareness and first responders, highly Communicable Diseases: Isolation/Quarantine trainings. FEMA student ID \#0004175749

- Assisted county wide mass vaccination efforts during a 2017-18 Hepatitis A outbreak. Duties included confirming patient vaccination histories using the Kentucky Immunization Registry; administering consent forms; and providing basic information about Hepatitis A, the ongoing outbreak, and information about the vaccination options.

Kentucky Hospital Association

Data Improvement Analyst

$2014-2015$

- Assisted in the development and management of federal, state and private grant funded hospital improvement databases for Kentucky hospitals to aid in reaching and maintaining state benchmarks in health care associated infections, readmissions, obstetrics harm and patient safety. Maintain daily and monthly uploads and data updates of all Kentucky Hospital Engagement Network databases. Communicate with hospital infection preventionist and support data collection protocol and question inquires. Generated statistical reports and summary analysis for maintained databases.

- Developed automated reporting system for Kentucky Hospital Engagement Network databases.

- Support the Kentucky Hospital Association in communications functions including member contact information database, weekly newsletters, webinars, hospital staff coaching, conference calls, meeting scheduling, maintaining the Kentucky Hospital Engagement Network website and resources and in-house calendar and communications.

Kentucky Cabinet for Health and Family Services- Kentucky Department for Public Health

Division of Healthcare-Associated Infections- Intern

$2013-2014$

- Supported the Kentucky Department for Public Health's division of Healthcareassociated infections in both epidemiological and biostatistical roles. 
- Worked on the development of a statewide Multidrug-resistant Organism surveillance system.

- Assisted in healthcare-associated infection outbreak investigations throughout the state of Kentucky and generated epidemiologic reports, which resulted in two Council of State and Territorial Epidemiology (CSTE) presentations.

- Contributed to the amendment process for the state disease surveillance regulation requiring mandatory reporting of multidrug-resistant organisms.

\section{Brown Forman Corporation}

\section{Quality Control (QC) Analyst, Research and Development (R\&D)}

- Obtained and maintained product compliant management records of all quality assurance actions. Quality assurance sample were tests collected and tested throughout processing from ingredient receiving to finished product specifications to variance testing of customer complaint specimens.

- Performed statistical process control surveillance analysis. Executed regular production line quality control checks as well as maintained federal regulatory affairs records of processing and analytical test data, details, inconsistencies and complications.

- Assisted Corporate R\&D Food Scientists with market driven product development for new alcohol-based beverage and beverage systems. Product development processes included analytical, sensory, financial and production consideration. Responsible for maintaining detailed dilution and scale-up processes, procedures, and records. Maintained a comprehensive flavors and analytical lab including equipment standardization and materials inventory.

Yum! Brands Inc.

\section{Corporate Kentucky Fried Chicken Research and Development (R\&D)- Intern 2010}

- Supported Yum! Brands Corporate research and development (R\&D) and quality assurance (QA) food scientists with new product development for the Kentucky Fried Chicken (KFC) system. Testing included analytical, sensory, nutrition, and operational process methodologies on all projects.

- Organized and executed product shows and brainstorming sessions for new product development. Maintained electronic records of testing and effectively communicated results to other corporate scientists and business professionals.

- Conducted competitive research on other quick service restaurants (QSR) to assist in corporate strategy.

Purdue University, Department of Food Science- Food Technology Development Laboratory

\section{Laboratory Technician}

- Conducted research on quality measurements of lipid coated shell eggs. Involved grading egg quality, $\mathrm{pH}$ analysis, shell strength, quantitative chemical analysis titrations, and microbiological assessment.

- Supported the development and implementation of proof of concept for an innovative in package sterilization plasma food treatment process. Performed 
ozone analysis and full microbial work-ups including agar and plate preparation, pour plates, dilutions, incubation, colony identification and plate counts.

\section{MANUSCRIPTS}

Stacey Konkle, Kristina Zierold, Kira Taylor, Daniel Riggs, Aruni Bhatnagar.

National Secular Trends in Ambient Air Volatile Organic Compound Levels and Biomarkers of Exposure in the United States. -Environmental Research March 2020

Stacey Konkle, Jordan Finch, Sanjay Srivastava, Russel Barnett, Aruni Bhatnagar.

Superfund Site Human Health Risk Assessment for Evaluating Sustainable Reuse Options. -In Preparation

\section{PEER-REVIEWED POSTER PRESENTATIONS}

Stacey Konkle, Jordan Finch, Aruni Bhatnagar, Katlyn McGraw, Haley Metcalf, Jamar Wheeler, Lauren Heberle. What Do We Know? A Critical Review of Site Condition and Public Health Risk Assessments in EPA Five-Year Reviews of the Lee's Lane Superfund Site in Louisville, KY. November 2019; Seattle, WA

Stacey Konkle, Kristina Zierold, Kira Taylor, Daniel Riggs, Aruni Bhatnagar. National Secular Trends in Ambient Air Volatile Organic Compound Levels and Biomarkers of Exposure in the United States. Presented at: The International Society for Environmental Epidemiology annual meeting. August, 2019- Utrecht, Netherlands; University of Louisville Research Symposium. September, 2019- Louisville Kentucky; Regional Society of Toxicology Annual Meeting. October 2019; Cincinnati, Ohio

Stacey Konkle, Kristina Zierold, Kira Taylor, Matthew Groenewold, Aruni Bhatnagar. Secular Trends in Ambient Volatile Organic Compound Levels, Biomarkers of Exposure and Associations with Cardiometabolic Syndrome: An Analysis of Data from the National Health and Nutrition Examination Survey (NHANES) and the National Monitoring Program. Presented at Kentucky Public Health Association annual meeting. April, 2019; Cincinnati, Ohio

Stacey Konkle, Kristina Zierold, Kira Taylor, Daniel Riggs, Aruni Bhatnagar. National Secular Trends in Ambient Air Volatile Organic Compound Levels and Biomarkers of Exposure in the United States. Presented at Cardiovascular Research Symposium. April, 2019; Louisville, Kentucky

Stacey Konkle, Kristina Zierold, Kira Taylor, Matthew Groenewold, Aruni Bhatnagar. Secular Trends in Ambient Volatile Organic Compound Levels, Biomarkers of Exposure and Associations with Cardiometabolic Syndrome: An Analysis of Data from the National Health and Nutrition Examination Survey (NHANES) and the National Monitoring Program (NMP). Presented at NIEHS Superfund Research Program (SRP) annual meeting. December, 2018; Sacramento, California

Stacey Konkle, Kristina Zierold, Kira Taylor, Matthew Groenewold, Aruni Bhatnagar. Secular Trends in Ambient Volatile Organic Compound Levels, Biomarkers of Exposure and Associations with Cardiometabolic Syndrome: An Analysis of Data from the National Health and Nutrition Examination Survey (NHANES) and the National Monitoring Program (NMP). Presented at 
Research Louisville Poster Symposium. September, 2018; Louisville, Kentucky - Doctoral Student Award Winner

Stacey Konkle, Kristina Zierold, Kira Taylor, Matthew Groenewold, Aruni Bhatnagar. Secular Trends in Ambient Volatile Organic Compound Levels, Biomarkers of Exposure and Associations with Cardiometabolic Syndrome: An Analysis of Data from the National Health and Nutrition Examination Survey (NHANES) and the National Monitoring Program (NMP). Presented at American College of Epidemiology Conference and published in Annals of Epidemiology. September, 2018; Cincinnati, Ohio

Stacey Konkle, Daniel Riggs, Aruni Bhatnagar. Associations Between Volatile Organic Compounds and Serum Lipids in the National Health and Nutrition Examination Survey (NHANES). Presented at Annual Kentucky Public Health Association Conference. April, 2018; Covington, Kentucky - Outstanding Student Epidemiology Poster Award Winner

Stacey Konkle, Daniel Riggs, Aruni Bhatnagar. Associations Between Volatile Organic Compounds and Serum Lipids in the National Health and Nutrition Examination Survey (NHANES). Presented at Society of Toxicology Annual Conference and Published in The Toxicologist. March, 2018; San Antonio, Texas

Stacey Konkle, Daniel Riggs, Aruni Bhatnagar. Associations Between Volatile Organic Compounds and Serum Lipids in the National Health and Nutrition Examination Survey (NHANES). Presented at Regional Society of Toxicology Annual Meeting. September, 2017; West Lafayette, Indiana

Stacey Konkle, Daniel Riggs, Aruni Bhatnagar. Associations Between Volatile Organic Compounds and Serum Lipids in the National Health and Nutrition Examination Survey (NHANES). Presented at Research Louisville Poster Symposium. September, 2017; Louisville, Kentucky - Doctoral Student Award Winner

Stacey Konkle, Daniel Riggs, Tim O'Toole, Wesley Abplanalp, Aruni Bhatnagar, Natasha DeJarnett, Shesh Rai. The Association of Benzene Exposure with Cardiovascular Disease Risk. Presented at Research Louisville Poster Symposium. October, 2016; Louisville, Kentucky

Wesley Abplanalp, Stacey Konkle, Daniel Riggs, Shesh Rai, Daniel Conklin, Sanjay Srivastava, Aruni Bhatnagar, Timothy O'Toole. Benzene Exposure is Associated with Insulin Resistance in Humans and Mice. Presented at Ohio Valley Society of Toxicology Annual Meeting Program. October, 2016; Indianapolis, Indiana

Matthew Groenewold, Stacey Konkle, Colleen Roberts, Andrea Flinchum, Lynn Roser, Derek Foster, Kimberly Blanton, Cibina Harris, Robert Brawley. Investigation of an Outbreak of Burkholderia Cepacia Infections Among Non-Cystic Fibrosis ICU Patients in a Kentucky Hospital. Presented at the Council of State and Territorial Epidemiologists 2015 Annual Conference. June, 2015; Boston, Massachusetts

Matthew Groenewold, Elizabeth Russell, Stacey Konkle, Joyce Rice, Kelly Giesbrecht, Heather Moulton-Meissner, Andrea Flinchum, Lynn Roser, Robert Brawley. Investigation of a Cluster of Rapidly-Growing Mycobacteria Infections Associated with Joint Replacement Surgery in a 
Kentucky Hospital. Presented at the Council of State and Territorial Epidemiologists 2014 Annual Conference. June, 2014; Nashville, Tennessee

Stacey Konkle, Austin Donner, Jean Jensen, Kevin Keener. Eradication of Salmonella enteritidis on Raw, Shell Eggs Using an In-Package Ozonation Process. Presented at the Purdue Undergraduate Symposium. April, 2010; West Lafayette, Indiana

\section{FORMAL PRESENTATIONS}

Stacey Konkle. National Secular Trends in Ambient Air Volatile Organic Compound Levels and Biomarkers of Exposure in the United States. Presented at The Envirome Institute Seminar Series. February, 2019; Louisville Kentucky

Stacey Konkle. Air Pollution: Global Burden, National Secular Trends, and Associations of Volatile Organic Compounds. Presented at post-doctoral seminar. November, 2018; Louisville Kentucky

Stacey Konkle. Volatile Air Pollutants: Trends, Associations, and Mitigation Strategies. Invited talk: The Kentucky Department for Public Health Career Development Webinar. July, 2018; Louisville Kentucky

Stacey Konkle. Trends in Ambient Volatile Organic Compound Levels, Biomarkers of Exposure and Associations with Cardiometabolic Syndrome. Presented at post-doctoral seminar. June, 2018; Louisville Kentucky

Stacey Konkle. Associations of Volatile Organic Compounds in Two Human Cohorts. Presented at post-doctoral seminar. February, 2018; Louisville Kentucky

Stacey Konkle. Associations of Volatile Organic Compounds and Cardiometabolic Syndrome Risk. Presented at post-doctoral seminar. July, 2017; Louisville Kentucky

Stacey Konkle. Characterization of Select Urinary Volatile Organic Compounds (VOCs) by Smoking Status in the Jackson Heart Study Cohort. Presented at the American Heart Association, Tobacco Regulation and Addiction Center (A-TRAC) annual meeting. March, 2017; Louisville Kentucky

Stacey Konkle. Volatile Organic Compound Exposure and Cardiometabolic Risk: An Analysis of Urinary Volatile Organic Compound Metabolite Exposures and Cardiometabolic Syndrome Risk. Presented at post-doctoral seminar. March, 2017; Louisville Kentucky

Stacey Konkle. Benzene Exposure and Cardiometabolic Syndrome: An Analysis of Benzene Metabolite Exposures and Cardiometabolic Syndrome Risk In a Nationally Representative Cohort. Presented at post-doctoral seminar. November, 2016; Louisville Kentucky

Stacey Konkle. The Association of Benzene Exposure with Cardiovascular Disease Risk. Presented at post-doctoral seminar. September, 2016; Louisville Kentucky

Stacey Konkle. Benzene Exposure and Cardiometabolic Syndrome. Presented at post-doctoral seminar. August, 2016; Louisville Kentucky 
Stacey Konkle. Analysis of VOC (Volatile Organic Compounds) Exposure and Cardiovascular Disease Risk Presented at post-doctoral seminar. February, 2016; Louisville Kentucky

\section{RECENT AWARDS AND HONORS}

Superfund Trainee selected to attend disaster research training. 2018

University of Louisville Superfund Center Fellow. 2018

Kentucky Public Health Association Outstanding Student Epidemiology Poster Award Winner. 2018

Research Louisville Poster Symposium Doctoral Student Award Winner. 2017, 2018

American Heart Association Tobacco Regulation and Addiction Center Affiliated Fellow. 2017

TRAINING

Probability Surveys SAS Certified (2018), NIEHS Superfund Research Program- Disaster Research Training (2018), NIEHS Superfund Research Program- Washington D.C. Science in Policy Training (2018), Louisville Metro Medical Reserve Corp.*, Legal Epidemiology (2018), Community Assessment for Public Health Emergency Response (CASPER) (2017), EpiInfor7 certified (2015), Kentucky Public Health Assistance and Support Team (KPHAST)*, FEMA ICS100* ICS200* and IS800* certified, HACCP certified, basic biosafety*, NIH guidelines*, radiation safety*, shipping infectious and biological substances*, general laboratory safety and hazardous waste safety*, bloodborne pathogens safety*, human subjects and HIPAA research certified*, good clinical practice training*.

*current

\section{SERVICE TO THE COMMUNITY}

St. Vincent de Paul

2011 - Present

- Dinner service at the soup kitchen, Christmas angel tree gift collection, Thanksgiving dinner delivery to families that have utilized St. Vincent de Paul services throughout the year, food and clothing drives, and additional assistance as needed.

\section{Southern Indiana Faith Community}

2011 - Present

- Set up and operate booths at several annual church picnics, organize fun-run fundraiser, food pantry food drives, packed shelf stable meals for Texas, Haiti, and Puerto Rico hurricane victims.

Kentucky Public Health Assistance and Support Team- Member

2013 - Present

- Trained with the Kentucky Department for Public Health to respond to public health situations and provide assistance with Incident Command Structure (ICS), survey design, Epi Info software, HIPPA, interviewing skills, and basic applied epidemiology.

- Deployed in the field to conduct a community assessment for public health emergency response (CASPER) in the Kentucky Green River Health District. CASPER is an epidemiologic tool designed to complete rapid needs assessment in communities. The CASPER system was used to systematically collect primary data for the districts next Community Health Assessment.

Habitat for Humanity

2016 - Present 
- Onsite build events - construction activities including painting, landscaping, siding, mudding, cleaning and various installations.

\section{PROFESSIONAL AFFILIATIONS}

International Society for Environmental Epidemiology (ISEE)

Certified Public Health (CPH)

Kentucky Public Health Association (KPHA)

American Statistical Association (ASA)

Society of Toxicology (SOT)

Ohio Valley Society of Toxicology (OVSOT)

Council of State and Territorial Epidemiologists (CSTE)

Society of Epidemiologic Research (SER)

American College of Epidemiology (ACE)

SKILLS

Proficient in SAS, SPSS, and EpiInfo7, competent in Esri ArcGIS, and R software, proficient in Microsoft Office including Excel, PowerPoint, Outlook and Word 